
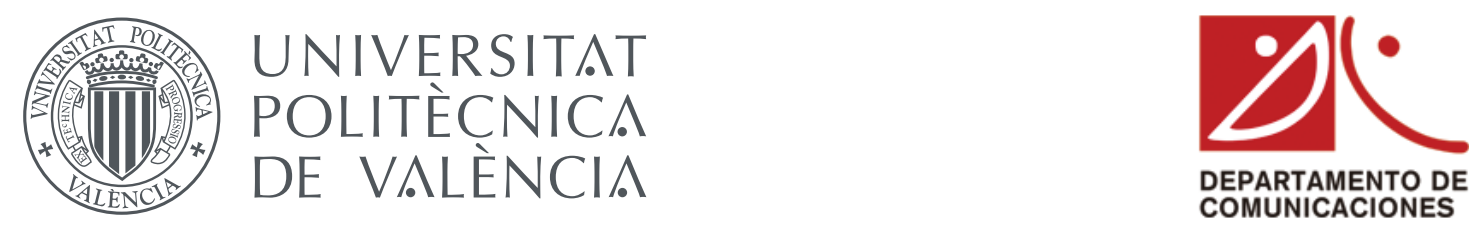

Doctoral thesis submitted for the degree of Doctor of Philosophy in Telecommunications Engineering

\title{
PHONONS MANIPULATION IN SILICON CHIPS USING CAVITY OPTOMECHANICS
}

\author{
Laura Mercadé Morales
}

Supervisor: Dr. Alejandro José Martínez Abiétar

Valencia, March 2021 
Ph.D. THESIS

Laura Mercadé Morales

Phonons manipulation in silicon chips using cavity optomechanics Nanophotonics Technology Center

UNIVERSITAT POLITÈCNICA DE VALÈNCIA 
A mi familia, en especial a mi hijo Ciro. 


\section{Abstract}

Cavity optomechanics deals with the interaction of light and matter through the radiation pressure effect, when the involved optical and mechanical waves are confined in a cavity. In optomechanical systems, photon and phonon interaction give rise to a plethora of phenomena as a function of the driving conditions of the system. Relative to that, two distinctive regimes can be obtained which enable either the absorption of phonons (cavity cooling) or their amplification (cavity heating). The first regime can be used to reduce the thermal occupancy of the system and it is commonly used for quantum processing information applications. However, the amplification of phonons, which can be performed at room temperature, has enabled to even reach phonon lasing conditions, a feature that could be used as a reference element for RF processing applications.

In this thesis, we address the simultaneous confinement and interaction of photons and phonons in periodic structures and unreleased waveguides on CMOS-compatible silicon-based technology. Throughout the experimental study of those periodic structures, we demonstrate that optomechanical cavities can perform as key blocks in the microwave photonics domain where all the information processing can be performed in the optical domain through phonon manipulation. In particular, we show that a single optomechanical oscillator can perform as both a local oscillator and an RF mixer, and it can operate as a frequency-converted of real data stream signals. To improve its performance, it is also demonstrated that optical frequency combs can be obtained by means of this system and multiple mechanical mode confinement can also be achieved, thus improving the functionality of the system. On the other hand, in order to fulfill the possible limitations of those systems, we explore different configurations enabling the simultaneous acousto-optic interaction together into the same structure. Especially, optomechanical interaction in high-index disks supporting quasi-bound states in the continuum is addressed, as well as a proposal of unreleased waveguides supporting strong Brillouin gains is also reported. The last one should lead to unreleased optomechanical interacting systems where the issue of phonon leakage into the substrate is solved, which could enormously simplify the fabrication of optomechanical systems in silicon chips as well as their practical use in multiple applications. 


\section{Resumen}

La optomecánica de cavidades se ocupa de la interacción entre la luz y la materia a través del efecto de presión de radiación cuando las ondas ópticas y mecánicas implicadas están confinadas en una cavidad. En estos sistemas optomecánicos, la interacción entre fotones y fonones da lugar a multitud de fenómenos en función de las condiciones en las que se excita el sistema. En particular, se pueden obtener dos regímenes distintos en los que se puede, o bien absorber fonones (denominado como enfriamiento de la cavidad), o bien éstos se pueden amplificar (régimen conocido como calentamiento de la cavidad). El primer régimen puede usarse, por ejemplo, para reducir la ocupación térmica del sistema y se usa comúnmente para aplicaciones relativas al procesado de información cuántica. Sin embargo, la amplificación de fonones, que puede ser desarrollada a temperatura ambiente, ha permitido conseguir alcanzar incluso las condiciones necesarias para obtener láseres de fonones, lo cual permite poder usar esta característica como elemento de referencia en aplicaciones relativas al procesado de señales de radiofrecuencia $(\mathrm{RF})$.

En esta tesis se aborda el confinamiento simultáneo y la interacción de fotones y fonones en estructuras periódicas y en guías no suspendidas desarrolladas en sistemas CMOS compatibles basados en tecnología de silicio. A través del estudio experimental de estas estructuras periódicas, hemos demostrado que las cavidades optomecánicas pueden actuar como elementos clave en el dominio de la fotónica de microondas, donde todo el procesado de la información puede ser realizado en el dominio óptico a través de la manipulación de fonones en este sistema. En particular, mostramos que un solo oscilador optomecánico puede actuar tanto como un oscilador local y un mezclador de $\mathrm{RF}$, y éste puede operar como un conversor de frecuencias de señales de cadenas de datos reales. Para mejorar esta funcionalidad, también se demuestra que es posible obtener tanto peines de frecuencias ópticos así como múltiples modos mecánicos confinados, aumentando así su rendimiento. Por otro lado, con el objetivo de poder solventar las posibles limitaciones de estos sistemas, en esta tesis también se exploran diferentes configuraciones que permiten la interacción acusto-óptica simultánea en la misma estructura. Específicamente, se analiza la interacción optomecánica en discos de alto índice que soportan estados cuasi-ligados en el continuo así como una propuesta de guías no suspendidas que soportan altas ganancias de Brillouin. Este último estudio debería permitir el desarrollo de sistemas optomecánicos no suspendidos donde el problema de la pérdida de fonones hacia el sustrato se resuelva, hecho que permitiría enormemente simplificar la fabricación de estos sistemas optomecánicos en chips de silicio así como su uso en múltiples aplicaciones. 


\section{Resum}

L'optomecànica de cavitats s'ocupa de la interacció entre la llum i la matèria a través de l'efecte de pressió de radiació quan les ones òptiques i mecàniques implicades estan confinades en una cavitat. En aquests sistemes optomecànics, la interacció entre fotons i fonons dona lloc a multitud de fenòmens en funció de les condicions de les condicions en les quals s'excita el sistema. En particular, es poden obtindre dos règims diferents en els quals es pot, o bé, absorbir fonons (denominat com a refredament de la cavitat), o bé, es poden amplificar (règim conegut com a calfament de la cavitat). El primer règim pot usar-se, per exemple, per a reduir l'ocupació tèrmica del sistema i s'usa comunament per a aplicacions relatives al processament d'informació quàntica. No obstant això, l'amplificació de fonons, que pot ser desenvolupada a temperatura ambient, ha permés aconseguir fins i tot les condicions necessàries per a obtindre làsers de fonons, la qual cosa permet poder usar aquesta característica com a element de referència en aplicacions relatives al processament de senyals de radiofreqüència $(\mathrm{RF})$.

En aquesta tesi s'aborda el confinament simultani i la interacció de fotons i fonons en estructures periòdiques i en guies no suspeses en sistemes CMOS compatibles basats en tecnologia de silici. A través de l'estudi experimental d'aquestes estructures periòdiques, hem demostrat que les cavitats optomecàniques poden actuar com a elements clau en el domini de la fotònica de microones, on tot el processament de la informació pot ser realitzat en el domini òptic a través de la manipulació de fonons en aquest sistema. En particular, vam mostrar que només un oscil-lador optomecànic pot actuar tant com un oscil-lador local i un mesclador de RF, i aquest pot operar com un convertidor de freqüències de senyals de cadenes de dades reals. Per a millorar aquesta funcionalitat, també es demostra que és possible obtindre tant tren de freqüències òptics així com múltiples modes mecànics confinats, augmentant així el seu rendiment. D'altra banda, amb l'objectiu de poder solucionar les possibles limitacions d'aquests sistemes, en aquesta tesi també s'exploren diferents configuracions que permeten la interacció acusto-òptica simultània en la mateixa estructura. Específicament, s'analitza la interacció optomecànica en discos d'alt índex que suporten estats quasi-lligats en el continu així com una proposta de guies no suspeses que suporten altes ganancies de Brillouin. Aquest últim estudi hauria de permetre el desenvolupament de sistemes optomecànics no suspesos on el problema de la pèrdua de fonons cap al substrat es resolga, fet que permetria enormement simplificar la fabricació d'aquests sistema optomecànics en xips de silici així com el seu ús en diverses aplicacions. 


\section{Acknowledgments}

I want to thank all the people at the Valencia Nanophotonics Technology Center who worked with me during these years and in particular to my Ph.D. supervisor Dr. Alejandro Martínez Abietar for his help and guidance, whose insight and knowledge into the subject guide me through this research.

I would also thank other NTC colleagues as Maria, David Zurita, Leo and Elena, for their help both within and beyond the lab. Thanks to all the colleagues that I met over these years that helped me all along this thesis as Kike, Evelyn, Sergio, Paula, Julio, Javi, Pablo, Alba, Irene, Alvaro, Diego, Miguel, Joannet, Luis, Maribel, David O. and Antoine. Thanks also to the all fabrication team, especially to Amadeu and Dora, for being always available to help.

I would like to thank Prof. Dr. Ewold Verhagen for his support and to give me the opportunity to join the Photonic Forces Group during my research stay at AMOLF. Also I want to thank the help and acceptance that all my AMOLF colleagues gave to me, specially Roel for his support in the lab and his patience with my Python skills but also to Javier, Jente, Rene, Jesse, Robin and Giada. I would also like thank Karl, from the University of Malta, bacuse your engagement and cooperation in the multimode project was crucial.

I may also noted that this work has been carried out under the framework of the H2020 FET-Open EU project PHENOMEN. I want to deeply thank all the partners that I met in the project for the warm reception and all the research feedback that they gave to me along all the project meetings. An special mention to Daniel Navarro who always was available to helped me with any technical question regarding the experiments. Also Bahram, Yan and Alexander from the University of Lille who always give me advice and guidance with the Brillouin theory and simulations.

I may also add that this Thesis was also supported by the Programa de Ayudas de Investigación y Desarrolo (PAID-01-16) de la Universitat Politècnica de València.

También me gustaría agradecer todo el apoyo que me han dado durante estos años tanto mi familia como mis amigos, en particular a Héctor, Reuben, Rocío, Denisse, Iaroslava e Isabel, por estar siempre disponibles y ayudarme cuando lo he necesito. También mención especial a mis padres, que me han apoyado tanto durante el desarollo de esta tesis como durante toda mi vida. Y por último, agradecer a mi pareja Rafa por acompañarme, apoyarme y ayudarme, desde lo emocional hasta con sus consejos más técnicos. 


\title{
List of abbreviations
}

\author{
OM OptoMechanical \\ OMC OptoMechanical Cavity \\ RF RadioFrequency \\ CMOS Complementary Metal-Oxide-Semiconductor \\ OFC Optical Frequency Comb \\ AO Acouto-Optic \\ SBS Stimulated Brillouin Scattering \\ TE Transverse Electric \\ TM Transverse Magnetic \\ PE Photoelastic \\ MI Moving Interface \\ ES Electrostriction \\ RP Radiation Pressure \\ SEM Scanning Electron Microscopy \\ PSD Power Spectral Density \\ OEO OptoElectronic Oscillator \\ OMO OptoMechanical Oscillator \\ EDFA Erbium Doper Fiber Amplifier \\ PD Photodetector \\ OSA Optical Spectrum Analyzer \\ RSA Radiofrequency Spectrum Analyzer \\ VOA Variables Optical Attenuator \\ LO Local Oscillator
}


xiv

IF Intermediate Frequency

DS Downconverted Signal

US Upconverted Signal

OFDM Orthogonal Frequency Division Multiplexing

SSO Single Self-Oscillation

MMO Multimode Self-Oscillation

BIC Boundary states In the Continuum 


\section{Contents}

Abstract $\quad$ v

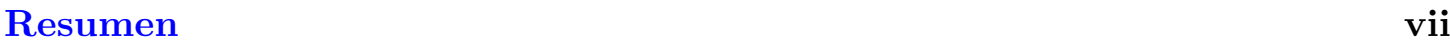

Resum $\quad$ ix

$\begin{array}{lc}\text { Acknowledgments } & \text { xi }\end{array}$

List of abbreviations $\quad$ xiii

1 Introduction $\quad \mathbf{1}$

1.1 Background and motivation . . . . . . . . . . . . . . . . . 1

1.2 Fundamentals of light-sound interaction . . . . . . . . . . . . . 4

1.2.1 Cavity-based optomechanics . . . . . . . . . . . . . 4

1.2.2 Travelling-wave stimulated Brillouin scattering . . . . . . . . 7

2 Optomechanics with a full phononic bandgap crystal cavity 9

2.1 Introduction . . . . . . . . . . . . . . . . . . . 9

2.2 OM crystal cavity design . . . . . . . . . . . . . . . . . . . 10

2.2.1 Photonic and phononic band diagrams . . . . . . . . . . . . 11

2.2.2 Acousto-optic interaction . . . . . . . . . . . . . . . 13

2.2.3 Fabricated OM cavity ................. . . 15

2.3 Optomechanical microwave oscillator . . . . . . . . . . . . . . 18

2.3.1 OMO phase noise .................. 21

2.3.2 RMS Jitter ....................... 23

2.3.3 Allan deviation ..................... 23

2.4 OM frequency comb generation . . . . . . . . . . . . . . . . 24

2.4.1 Temporal OFC dynamics . . . . . . . . . . . . . . 27

2.5 Experimental setups . . . . . . . . . . . . . . . . . 29

2.5.1 Optical and mechanical cavity characterization . . . . . . . . . 29

2.5.2 Optomechanical coupling rate measurement . . . . . . . . . . 30

3 All-optical frequency conversion $\quad 35$

3.1 Introduction . . . . . . . . . . . . . . . . . . . 35

3.2 Long-term stability . . . . . . . . . . . . . . . . . . . . 37

3.3 Frequency conversion in the lasing regime . . . . . . . . . . . . 38

3.3.1 Data stream modulation using OFDM . . . . . . . . . 39

3.3.2 Downconversion using the first harmonic of the mechanical resonance 40

3.3.3 Upconversion for higher order harmonics . . . . . . . . . . 43 
3.4 Experimental setup . . . . . . . . . . . . . . . . . . . 46

3.4 .1 OFDM Frequency conversion setup . . . . . . . . . . . 46

4 Multimode phonon lasing in an OMC $\quad 47$

4.1 Introduction . . . . . . . . . . . . . . . . . . . . 47

4.2 Multiple mechanical mode confinement . . . . . . . . . . . . . . . . . 48

4.2.1 Phononic band diagram approach . . . . . . . . . . . . . . . 49

4.2 .2 Individual single-mode phonon lasing . . . . . . . . . . . . . . 52

4.3 Multimode phonon lasing . . . . . . . . . . . . . . . . 53

4.3.1 Driving the multimode lasing state . . . . . . . . . . . . 54

4.3.2 Comparative stability analysis . . . . . . . . . . 58

4.4 Experimental setup . . . . . . . . . . . . . . . . . . 61

4.4.1 Multimode oscillation driving setup . . . . . . . . . . . 61

$5 \quad$ High-index disk optomechanics of supercavity modes $\quad 63$

5.1 Introduction . . . . . . . . . . . . . . . . . 63

5.2 Quasi-BIC modes in high-index disks . . . . . . . . . . . . . . . . . 64

5.2 .1 Scattering cross section . . . . . . . . . . . . . . . 64

5.2 .2 Optomechanical coupling . . . . . . . . . . . . . 65

5.3 Pedestal's substrate mechanical leakage . . . . . . . . . . . . . 67

6 Strong light-sound interaction in unreleased Si-based waveguides $\quad \mathbf{7 1}$

6.1 Introduction . . . . . . . . . . . . . . . . 71

6.2 Description of the light - sound waveguiding system . . . . . . . . 73

$6.3 \mathrm{AO}$ interaction and Brillouin gain . . . . . . . . . . . . 76

6.4 Intramodal first order mode interaction . . . . . . . . . . . . . 77

6.4.1 Forward Stimulated Brillouin Scattering . . . . . . . . . . . . 77

6.4.2 Backward Stimulated Brillouin Scattering . . . . . . . . . . . 80

6.5 Enhancement of the Brillouin gain at low temperature . . . . . . . . . . 82

6.6 Practical implementation . . . . . . . . . . . . . . . 82

$\begin{array}{lll}7 & \text { Conclusions and perspectives } & 85\end{array}$

$\begin{array}{ll}\text { Appendices } & 91\end{array}$

A $\quad$ OM crystal cavities fabrication . . . . . . . . . . . . . . . 92

B Taper loop fabrication . . . . . . . . . . . . . . . . . 93

C OFDM signal description . . . . . . . . . . . . . . 96

D Author's merits . . . . . . . . . . . . . . . . . . 97

D.1 Publications . . . . . . . . . . . . . . . . . . . 97

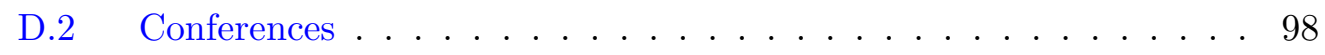

$\begin{array}{lr}\text { Bibliography } & 100\end{array}$ 


\section{Chapter 1}

\section{Introduction}

\section{Contents}

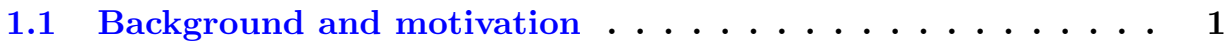

1.2 Fundamentals of light-sound interaction . . . . . . . . . 4

1.2.1 Cavity-based optomechanics . . . . . . . . . . . . . 4

1.2.2 Travelling-wave stimulated Brillouin scattering $\ldots \ldots \ldots .7$

\subsection{Background and motivation}

In the 17th century, the observation that the dust tails of a comet always point away from the Sun led Johannes Kepler to put forward the radiation pressure concept [1]. Radiation pressure is thus understood as a mechanical pressure applied upon a surface by the momentum carried by the light [2], that is, the light force exerted by any electromagnetic field interacting with either macroscopic or microscopic objects. Throughout history, these forces have been a key point in the demonstration of innovative developments including measurement sensitivity increments by coupling optical and mechanical modes [3], trapping and manipulation of dielectric particles by laser beams [4], experimental proposals for quantum noise reduction measurements [5,6], applications as atomic clocks [7] or even cavity-assisted laser cooling systems used to cool down the motion of atoms and molecules $[8,9]$.

All the research involving the effects of light-matter interaction when mediated by the aforesaid radiation pressure effect has evolved to give birth to the discipline known as cavity optomechanics. Cavity optomechanics addresses the interaction between light and mechanical waves when confined in a cavity $[2,10]$. This interaction can give rise to a plethora of intriguing phenomena such as quantum ground-state cooling [11], quantum squeezing of the light field $[12,13]$, mechanical entanglement [14], phonon lasing [15, 16], optomechanically induced transparency [17, 18], or non-reciprocal behavior [19]. Moreover, optomechanical (OM) systems hold also the promise to be used as optically driven mechanical sensors [20,21].

Remarkably, optomechanical $(\mathrm{OM})$ interaction can be observed in a wide variety of systems. Indeed, multiple technological platforms implementing OM cavities can be found in the literature, ranging from optical microtoroids resonators [22-24], micromechanical membranes [25], or whispering gallery modes in microspheres and microdisks [26-28] to optomechanical cavities (OMC) lithographically defined on released 
high-index nanobeams, the so-called OM crystals [29-31]. A selection of different OM devices is shown in Fig. 1.1 over a wide range of sizes from millimeter to micrometer scales.
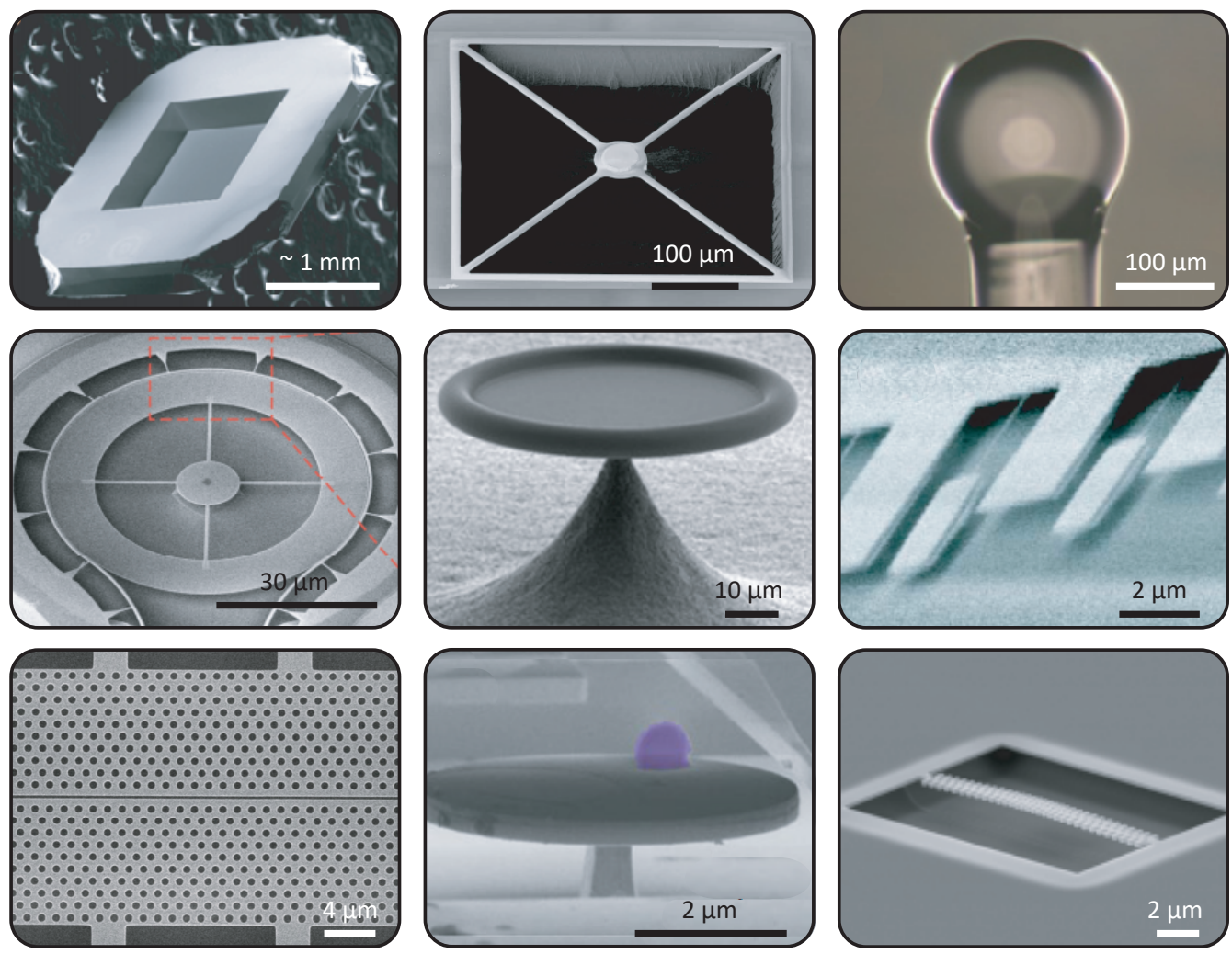

Figure 1.1: Selection of optomechanical systems, from left to right: (Top row) Suspended micromechanical membrane [32]. Trampoline resonator [33]. Optical microsphere resonator [34]. (Middle row) Wheel-shaped resonator [35]. Microtoroidal optomechanical cavity [36]. Cantilever suspended micromirror [37]. (Bottom row) Two-dimensional photonic crystal cavity [38]. Optomechanical disk resonator [20]. One-dimensional optomechanical crystal cavity [31].

Besides the referred cases, applications involving phonon manipulation in OMCs could also be used in microwave photonics, a field that involves the processing of radiofrequency (RF) signals entirely in the optical domain [39]. Photonics-based processing of microwave signals presents multiple advantages compared to the processing of such signals in the electric domain, including nearly-unlimited bandwidth, immunity to electromagnetic interference, or frequency-independent losses [40]. In comparison to standard microwave photonics based on elements such as semiconductor optical amplifiers or Mach-Zehnder modulators, OMCs offer the possibility to process microwave signal in wavelength-scale footprints [40], mainly if the cavities are built using OM crystals. An extra advantage could arise if the OM employed devices could be fabricated using complementary metal-oxide-semiconductor (CMOS) technology since it could take advantage of the well-established fabrication processes, thus enabling low-cost manufacturing devices.

Recent years have witnessed a series of preliminary demonstrations of the use of OM cavities as photonics-based elements for processing of microwave signals, including RF 
down-converters [41,42], RF mixers [43], or microwave oscillators [44]. However, most of them were demonstrated for mechanical oscillators in the $\mathrm{MHz}$ regime [41-43] while the ones in the $\mathrm{GHz}$ regime were not silicon CMOS compatible [44]. Oscillators working in the $\mathrm{GHz}$ radio spectrum range are of relevant importance for practical applications as it is the range where most of the telecommunications bands operate. For instance, wireless WiMAX bands [45], the commercial 5G new-radio bands [46], or even the X-band used for radar or satellite communication. Additionally, due to the massive deployment of such technologies, the realization of photonic devices in silicon technology would be a clear benefit over non-CMOS competitors.

Therefore, a major goal in this field is the realization of an OM system working at those $\mathrm{GHz}$ frequencies that could act as an OM oscillator with competitive performance whilst being CMOS-compatible. In this thesis, in Chapter 2, we experimentally demonstrate an OM crystal cavity design that can act, in a so-called phonon lasing regime, as a $\mathrm{GHz}$ oscillator with low phase noise values, in comparison with commercial devices. Moreover, since these OM systems are nonlinear elements, it has been demonstrated that multiple harmonics can be obtained in the optical signal [22,23,41]. Therefore, multiple harmonic generations of RF tones enable access to higher frequency bands, resulting in a promising structure for microwave applications, a feature that is also explored up to $20 \mathrm{GHz}$. To demonstrate a reliable route towards all-optical microwave processing in silicon cavities, in Chapter 3, the performance of the OM cavity acting simultaneously as a local oscillator and an RF mixer is demonstrated to perform frequency conversion of real data stream signals compliant with a well-known wireless communications standard.

However, in both Chapters 2 and 3, only a configuration with a single optical and a mechanical mode is attained, but the manipulation of multiple mechanical modes could potentially lead to achieve novel functionalities. For instance, multimode phonon lasing has broad significance because of the emergent phenomena in multimode self-oscillating systems, including synchronization [47-53], stability enhancement [54], dynamical topological phases [55], and analog simulators [56]. Consequently, in Chapter 4, a route to design OM crystal cavities with multiple mechanical modes is addressed and it is demonstrated that phonon lasing regimes into the same physical structure can be obtained, thus increasing the capabilities. Moreover, multimode mode-locked phonon lasing is demonstrated by modulating the driving laser by a low-frequency RF signal. Notably, mode-locking results in a significant reduction of the phase noise of the oscillators, in agreement with other mode-locked systems.

Besides that, in this thesis, we also explore two other configurations where the light and sound interaction plays a crucial role as is the case of the supercavity modes in high-index dielectric disks and the acousto-optic Brillouin interaction in silicon-based waveguides. In the field of high-index disks, it has been demonstrated that far-field scattering can be canceled out which enables tight localization of the optical field inside the structure just by properly tailoring their aspect ratio, arising the termed quasibound states in the continuum $[57,58]$. This results in relatively high values of the optical quality factor, Q, which can be above 100, as well as in large field densities inside the disk, making them highly appropriate to enhance nonlinearities. However, whilst nonlinearities based on the Kerr effect have been studied in such disks, there have not been studies about the OM interaction. In Chapter 5, the OM interaction between high$\mathrm{Q}$ optical modes and the free mechanical modes of high-index disks is analyzed. The large OM interaction found in these system makes such disks an appealing route to build massive arrays of OM cavities for GHz-tunable OM metasurfaces. 
Finally, in Chapter 6, we introduce an unreleased silicon-based waveguide that can be lithographically defined to confine both optical and mechanical waves in the silicon core with strong interaction. We engineer the waveguide vertically by inserting a thick silicon nitride layer between the top silicon waveguide and the silica substrate which enormously reduces phonon leakage and enables strong localization of the mechanical field at room temperature. The main motivation under this study is that most of the OM cavities studied along with this thesis and the ones that can be found in the literature made use of released ultra-thin silicon membranes to prevent mechanical leakage into the substrate $[30,31,59,60]$. However, the obtainment of practical approaches where this phonon leakage can be prevented without releasing the structures may be of high impact [61].

Previous to the presentation of the obtained results, a brief introduction to the fundamentals of light-sound interaction is presented in this introductory chapter. Both from the cavity-based optomechanics and the Brillouin scattering perspectives. There is a double purpose for this: to present the similarities and fundamentals concepts concerning these topics and to define the working conditions that will be applied to our systems.

\subsection{Fundamentals of light-sound interaction}

Despite both cavity optomechanics and Brillouin scattering account for the interaction between light and sound, they were historically presented and treated as independent phenomena. Certainly, some differences made them independently evolve in separate fields, as their distinct phonon group-velocities or the differences related to the phonon/photon dissipation channels [62]. However, the key point of the two lightsound interacting pictures lies in the same concept: first, the light generates motion that phase-modulates the initial light, then, this phase-modulation generates sidebands that, finally, interfere with the field already present in the system [63]. Essentially, this feedback loop is the driving mechanism of both configurations but one is studied in cavities, the cavity-based optomechanics, and the other is driven in waveguides, the traveling-wave Brillouin scattering. Therefore, even though we address in this section and all along with the thesis both configurations independently, the underlying physics of the driving conditions is known to be the same.

\subsubsection{Cavity-based optomechanics}

Figure 1.2(a) depicts a canonical representation of an OMC: a Fabry-Pérot cavity with two highly reflective mirrors. One of them is mounted on a spring, thus enabling the mirror to move, while the other is fixed. Through the fixed mirror, an input light field (at $\omega_{L}$ frequency) enters the cavity by virtue of its partial reflectivity, giving rise to an optical cavity mode $\widehat{a}$ at the resonance frequency $\omega_{o}$ for the undeformed cavity of length $L_{\text {eff }}$. Then, the impinging light on the movable mirror, by means of the radiation pressure effect, puts the mass-spring system into motion, thus resulting in a mechanical mode $\widehat{b}$ at the oscillation frequency $\Omega_{m}$. A change in the position, $\widehat{x}$, of the movable mirror, alters the cavity length, and thus the optical resonance conditions. That means that the optical confined mode shifts in frequency by the amount [64]:

$$
\Delta \omega_{o}=\frac{\partial \omega_{o}}{\partial x} \widehat{x}
$$


Therefore, the momentum carried by the optical field puts a mechanical oscillator into motion that, at the same time, induces a change in the optical properties of the cavity. These changes can be related, for instance, to the circulating light intensity or the detuning with respect to the input field $\Delta=\omega_{L}-\omega_{o}$ [2]. Also, the finite lifetime of the photons inside the cavity $\tau$ introduces a delay between the motion and the resulting changes of the force. This feedback loop, of paramount importance in many physical systems, is known as OM dynamical backaction [2,10]. On other side, the strength of this interaction can be measured with the vacuum OM coupling rate given by [65]:

$$
g=x_{z p f} \frac{\partial \omega_{o}}{\partial x}
$$

where $x_{z p f}$ is the zero-point fluctuation usually defined as $x_{z p f}=\sqrt{\hbar / 2 m_{e f f} \Omega_{m}}$, with $m_{e f f}$ as the effective mass of the mechanical oscillator (the movable mirror in the canonical picture).

(a)

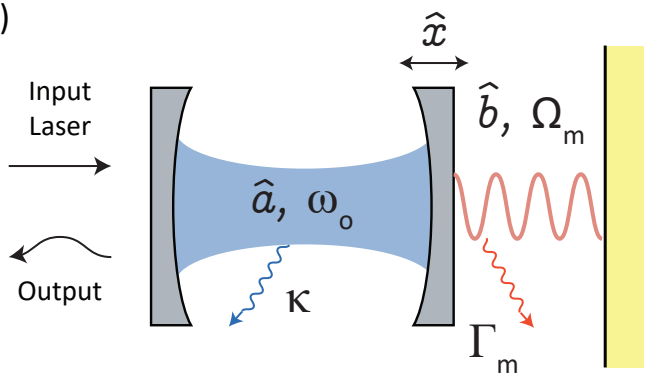

(b)

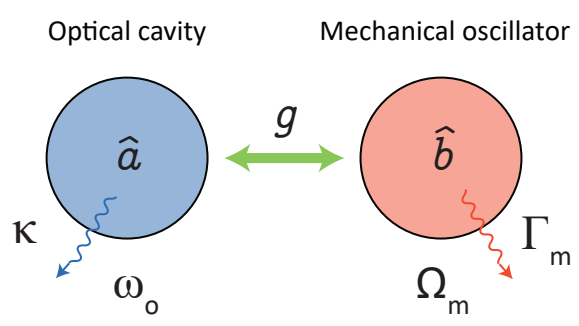

Figure 1.2: (a) Canonical optomechanical Fabry-Perot cavity with a movable mirror connected to a spring. The system results in a confined optical cavity mode $\widehat{a}$ and a mechanical oscillator $\widehat{b}$ at their resonance frequencies $\omega_{o}$ and $\Omega_{m}$. (b) Optomechanical linearized interaction picture between an optical and a mechanical mode with a coupling strength $g$.

It must be noted that both the optical and the mechanical modes have their loss channels, which can be described by the loss rate. The total optical cavity loss rate is given by $\kappa$, which is the sum of both the external $\left(\kappa_{e}\right)$ and internal $\left(\kappa_{i}\right)$ loss rates. Here, $\kappa_{e}$ represents the couling to the outer world whilst $\kappa_{i}$ stands for the intrinsic losses (absorption, fundamentally) inside the resonator. The optical loss rate is related with the photon lifetime as $\kappa=1 / \tau$ and it allows the calculation of the optical quality factor $Q_{o}$, which can be defined as $Q_{o}=\omega_{o} / \kappa$. Concerning the leakage of mechanical energy, it is described by the damping rate $\Gamma_{m}$, which is related to the mechanical quality factor as $Q_{m}=\Omega_{m} / \Gamma_{m}$. Alternatively, another common picture of the OM interaction involving an optical cavity and a mechanical oscillator is given in Fig. 1.2(b), where the main mode parameters are also depicted.

As noted in the previous section, $\mathrm{OM}$ interaction is driven by the radiation pressure, which can be understood as the light force exerted by an optical field interacting with either macroscopic or microscopic objects. However, it must be pointed out that since optical forces tend to be faint due to the small momentum of photons, structures designed to enhance the OM interaction need to be used to produce observable effects, as the structures mentioned in the last section (see Ref. [2] for a broad overview). Through these $\mathrm{OM}$ structures, observable $\mathrm{OM}$ interaction effects are obtained, which can be 


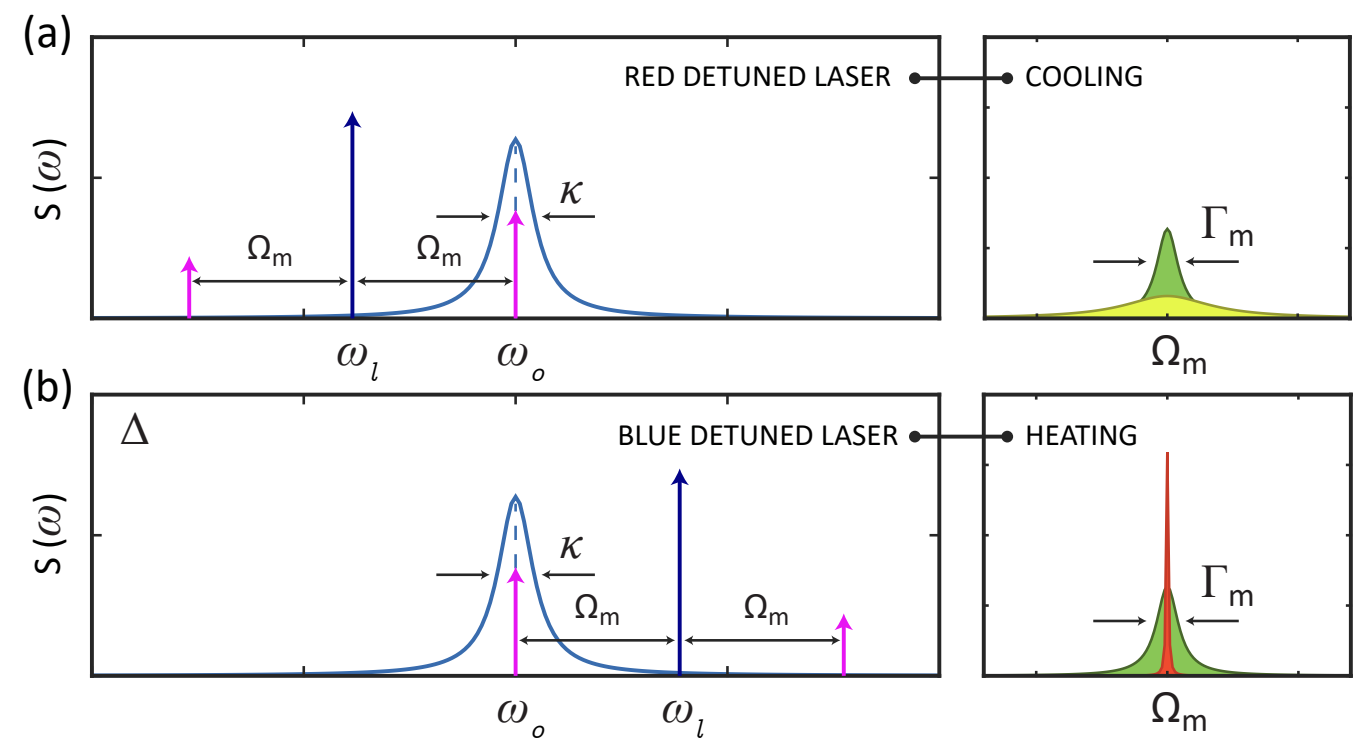

Figure 1.3: (a) Power spectral density picture of the OM interaction with a laser input field red-detuned with respect to the optical cavity. For a non-zero laser detuning, the intracavity field exhibits two non-symmetric sidebands as a result of energy conservation arguments, leading to absorption of phonons or cavity cooling. (b) Power spectral density picture with a blue-detuned laser input field. In this situation, for a non-zero laser detuning, dynamical back-action leads to amplification of phonons or cavity heating.

experimentally measured by the output light field emerging from the cavity, as in Fig. 1.2(a). In a spectral representation, as in the left panels in Fig. 1.3, output photons exhibit sidebands at frequencies $\omega_{L} \pm \Omega_{m}$, as a result of the scattering process, which will be more effective if such output photons resonate in the cavity (as a result of the large density of states inside the cavity). Different regimes can be distinguished depending on the relation between the optical loss rate and the mechanical frequency. If $\Omega_{m}>\kappa$, we enter in the so-called resolved sideband regime (also called the good cavity limit), where the mechanical frequency is comparable to or even exceeds the optical decay rate. However, in the situation when $\Omega_{m}<\kappa$ we work in the unresolved sideband regime (or bad cavity regime). Besides these cases, if the laser is red-shifted with respect to the cavity and $\Delta=\Omega_{m}$, the OM interaction will result in the absorption of phonons (cavity cooling), as it is depicted in Fig. 1.3(a). Under suitable circumstances, this can lead to reaching the quantum-ground state (less than one phonon on average) in the cavity [11,66]. On the contrary, if the laser is blue-shifted by $\Omega_{m}$, phonons are created inside the cavity, which leads to phonon amplification and, ultimately, phonon lasing [67,68], as in Fig. 1.3(b). In this Thesis, we will put particular attention to the blue-detuned regime: we will work in a regime where the phonons in the cavity will be amplified, giving rise to the previously mentioned phonon lasing regime. This will allow us to generate coherent $\mathrm{GHz}$ phonons that will be used to process microwave signals in the optical domain. 


\subsubsection{Travelling-wave stimulated Brillouin scattering}

Brillouin scattering can be understood as the inelastic scattering process to convert a photon to a photon with lower energy such that the energy difference appears in the form of an acoustic phonon [69]. Therefore, it is also an AO or OM process involving interaction between light and mechanical waves. It was first predicted by Léon Brillouin in 1922 [70], leading to the so-called Brillouin scattering. In the case when a strong intensity-modulated field is the one that generates the effect, the nonlinear process is then known as Stimulated Brillouin Scattering (SBS), which was experimentally demonstrated in 1964 [71]. Apart from optical fibers [72-74], where SBS can be observed at input power around $10 \mathrm{~mW}$, over the past years SBS has gained a lot of attention in other integrated waveguide systems as rib-waveguides [75,76], suspended waveguides $[77,78]$ or hybrid photonic-phononic membranes [60,79]. In particular, in this thesis, we explore the occurrence of SBS in silicon-based integrated waveguides without requiring the release of the waveguide core to prevent phonon leakage.

(a)

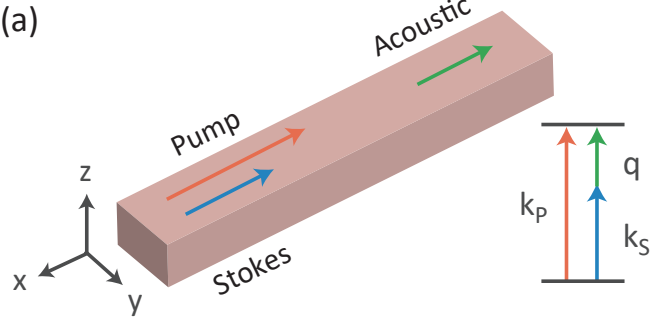

(b)

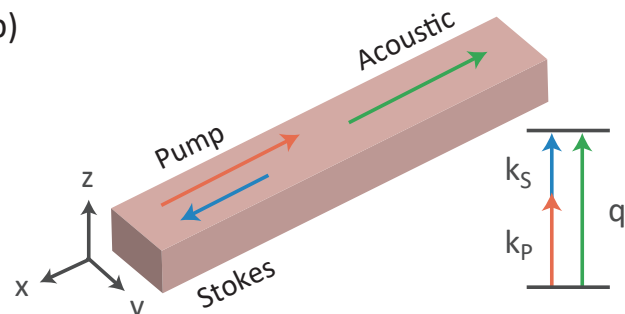

(c)

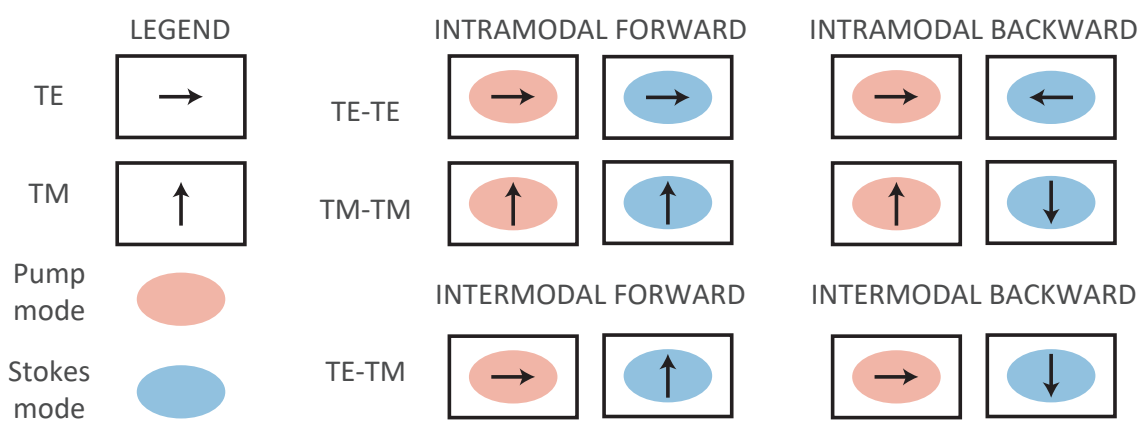

Figure 1.4: (a) Forward waveguide and vector phase matching diagram. (b) Backward waveguide and vector phase matching diagram. (c) Different SBS configurations involving two first order TE and TM optical waves.

Therefore, the SBS phenomenon is a third-order nonlinear process arising from photon-phonon interaction in material systems supporting both optical and acoustic propagating waves $[70,71]$, by which optical photons are coupled to acoustic phonons $[69,80]$. It is usually obtained through the interaction of two optical waves, named pump and Stokes waves, whose interference generates dynamic optical forces at the beat frequency $\omega_{p}-\omega_{s}=\Omega_{m}$ [81]. As a function of the propagation direction of the involved optical waves and related with its momentum conservation, two different Brillouin scattering situations can be identified. When the pump and Stokes waves propagate codirectionally, as depicted in Fig. 1.4(a), it is the so-called Forward SBS, where the phasematching condition leads to an acoustic mode at the wavevector given by $q=k_{p}-k_{s}$. Conversely, once the incident pump and the Stokes wave propagate contra-directionally, 
the resulting acoustic mode is generated at $q=k_{p}+k_{s}$ by the conservation of momentum. The process is then known as Backward SBS, as depicted in Fig. 1.4(b). However, it must be noted that the optical modes related to this process can be either equal or different in terms of polarization (Transverse Electric (TE) or Transverse Magnetic (TM) modes) or they can even be the same or different order modes. Figure 1.4(c) shows different SBS configurations with first TE and TM order modes in both intramodal (pump and Stokes modes are equal) and intermodal (pump and Stokes modes are different) interactions. Notice that the wave vector conservation arises from the fact of having a system with continuous translational symmetry along the propagation direction. This makes a huge difference with respect to the case of cavity optomechanics, where no rules are imposed over the momentum of either the optical or mechanical waves. Besides this fact, SBS shows many resemblances with cavity optomechanics, as well explained in Ref. [62], though instead of having confined waves (cavity optomechanics) in SBS we play with propagating modes.

The resulting interaction strength will rely upon the temporally modulated optical force distributions $\mathbf{f}$ generated by the two involved optical modes interacting. These forces distributions can have either electrostrictive nature (related with the bulk material properties) or they can come from the radiation pressure effect (playing a huge role in the boundary-induced force profiles) [77] and will rule the magnitude and distribution of the photon-phonon coupling process. The overlap between the optical forces and the mechanical modes of frequency $\Omega_{m}$ at the allowed acoustic wavevector $q$ will determine then the Brillouin gain coefficient $G_{B}$. Here, however, we only present the overall picture that will be addressed broadly in Chapter 6 , where both the coupling equations and the distributed field profiles and the Brillouin gain will be discussed for our proposed configuration. 


\section{Chapter 2}

\section{Optomechanics with a full phononic bandgap crystal cavity}

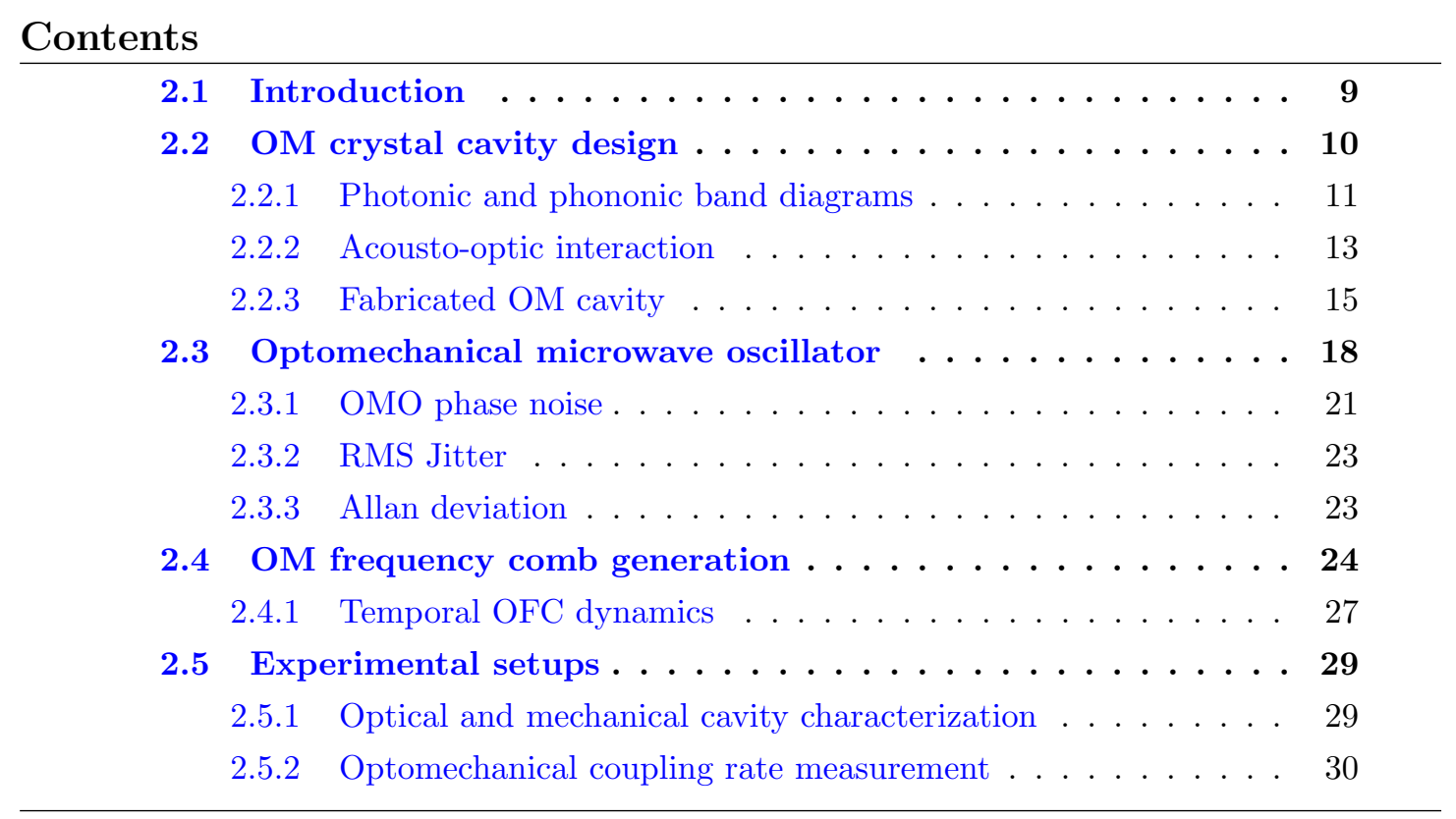

\subsection{Introduction}

Since mechanical vibration can efficiently modulate the intensity of an input optical signal via dynamical back-action [82], OM cavities could play a role in microwave photonics, a discipline that addresses the processing of microwave signals in the optical domain [39]. The great advantage of using phonons - or mechanical vibration - instead of electrical signals is that the wavelength is reduced by about five orders of magnitude. Indeed, this is the reason why acoustic filters based on interdigital transducers are massively used in mobile phones and other wireless systems and devices. Some experiments have shown the performance of OM cavities as radiofrequency (RF) down-converters [41] or OM oscillators [44], which are essential functionalities in microwave photonics. Moreover, since OM cavities are nonlinear elements, multiple harmonics of the fundamental mechanical vibrations can be over imposed on the optical signal [22,23,41], a phenomenon that has 
been recently interpreted theoretically as an optical frequency comb (OFC) [83]. Notably, recent experiments have also shown that OFCs generated by Kerr nonlinearities could also play a role in the processing of microwave signals in the optical domain [84].

In this chapter, we first demonstrate an OM cavity on a silicon nanobeam having a breathing mechanical mode vibrating close to $4 \mathrm{GHz}$ with a high $g_{0}$ and placed in a full phononic bandgap. To that end, the fundamental notions about the cavity design are first presented, followed by a detailed analysis of the resulting fabricated structure. Then, by driving the cavity with a blue-detuned laser, we demonstrate phonon lasing of the fundamental mechanical mode. We measure the phase noise of the generated microwave tone and show that the cavity can perform as an OM-based microwave oscillator. Stronger pumping and detuning of the cavity leads to the generation of a series of harmonics forming an OFC of OM nature, whose phase noise degrades with the harmonic number as in standard harmonic mixing. Real-time measurements of the temporal traces that show a good agreement with a theoretical model of an OFC are also presented. This confirms that OM cavities can be used for the generation of OFCs with GHz-scale line spacing and may find application as ultracompact and lightweight processing elements for microwave photonics.

\subsection{OM crystal cavity design}

OM crystal cavities can be created on released silicon nanobeams with one-dimensional (1D) periodicity. The key idea is to have OM mirrors (that prevent leakage of both photons and phonons) on each nanobeam side whilst the main parameters of the periodic structure (such as the period or the size of the holes) are adiabatically changed towards its center to allocate confined modes. Moreover, the cavity has to be designed to ensure a good overlap between the optical and mechanical resonant modes to produce a sufficiently large OM coupling rate $g_{0}$. Notice that the whole nanobeam has to be released in order to prevent phonon leakage towards the silica substrate, which happens because the speed of sound is lower in silica than in silicon.

One of the most popular 1D OM cavities in silicon nanobeams [30] consists of a series of elliptic holes whose size and the axial ratio is adiabatically modulated. This OM cavity exhibits values of $g_{0} / 2 \pi \approx 1 \mathrm{MHz}$ but it does not have a full phononic bandgap but a partial one, because a full phononic bandgap cannot be obtained by merely drilling holes in the nanobeam [85]. In order to reduce phonon leakage, the cavity can be surrounded by a two-dimensional (2D) "acoustic shield", which is basically a $2 \mathrm{D}$ structure exhibiting a complete phononic bandgap at the required frequency [30]. This hybrid 1D/2D approach has been successfully applied in a number of experiments, such as the exploration of nonlinear dynamics [86], the cooling down to the quantum ground state [11,87], the phonon guidance through waveguides [88] and, more recently, the demonstration of ultrahigh mechanical quality factors $Q_{m} \approx 10^{10}$ in cryogenic environments [89].

Having a full phononic bandgap in a $1 \mathrm{D}$ silicon nanobeam requires making lateral corrugations in addition to the holes [85]. Using this approach, the existence of mechanical modes in a full phononic bandgap of a 1D OM crystal consisting of circular holes and lateral wings was demonstrated [31]. However, the mechanical modes within the bandgap in the cavity demonstrated in Ref. [31] exhibits low $g_{0} / 2 \pi$ values, being the breathing mechanical mode located out of the phononic bandgap. As a result, the excitation of these modes is not efficient and, in general, all injected energy goes to the nanobeam flexural modes (oscillating at tens of $\mathrm{MHz}$ ), which has been successfully used 
to demonstrate phonon lasing [90], chaotic dynamics [91] and, more recently, synchronization [51]. This basic OM structure can be further engineered so that breathing-like mechanical modes appear within the full bandgap, as shown in Ref. [92].

In this section, we show the photonic and phononic band diagrams of the nominal optomechanical cavity optimized in order to fulfill our requirements, the optomechanical coupling coefficient of the modes involved, and the analysis of the resulting fabricated structure with its fabrication imperfections.

\subsubsection{Photonic and phononic band diagrams}

In a periodic dielectric media, such as in photonic crystals, the propagation of light is governed by Maxwell's equations. If there are no currents or free charges, we have:

$$
\begin{aligned}
& \nabla \cdot \mathbf{D}=0, \quad \nabla \times \mathbf{H}-\frac{\partial \mathbf{D}}{\partial t}=0 \\
& \nabla \cdot \mathbf{B}=0, \quad \nabla \times \mathbf{E}-\frac{\partial \mathbf{B}}{\partial t}=0
\end{aligned}
$$

where $\mathbf{E}$ and $\mathbf{B}$ are the electric and magnetic fields and $\mathbf{D}$ and $\mathbf{B}$ are the displacement and magnetic induction fields, respectively. In the case of linear equations and for a periodic system, we can decompose the previous fields into a set of harmonic modes once applying Bloch's theorem. In such a way (for more details see Ref. [93]), we can reduce the electromagnetism problem to a master equation given by:

$$
\nabla \times\left(\frac{1}{\varepsilon(\mathbf{r})} \nabla \times \mathbf{H}(\mathbf{r})\right)=\left(\frac{\omega}{c}\right)^{2} \mathbf{H}(\mathbf{r})
$$

where $c$ is the speed of light.

This results in an eigenvalue problem where the solutions will be the possible electric field profiles (as eigenvectors) at a given frequency (as eigenvalues). As we are dealing with a periodic problem, we can restrict the analysis of the eigenvalue solutions $\omega_{m}(\mathbf{k})$ to the irreducible Brillouin zone where $\mathbf{k}$, the propagation wavevector, will be analyzed in the range of $[0, \pi / a]$ in the propagation direction. Here, $m$ is the band number and $a$ is the lattice constant. The information of the set of function families $\omega_{n}(\mathbf{k})$ results in the so-called band diagram, which provides information about the allowed and forbidden frequency states for a given periodic structure.

Therefore, in order to build the cavity, we first examine the photonic band diagram of the mirror unit cell, which is presented in Fig. 2.1(a). As we are working with a three-dimensional (3D) geometry, the dielectric constant depends on the three coordinates. This results in a hybridization of the modes in Maxwell's equations because the separation of Maxwell's equations into transverse electric (TE) and transverse magnetic (TM) polarization is only possible for $1 \mathrm{D}$ and 2D problems [64,93]. However, as our structure has different planes of symmetries (as $z=0$ or $y=0$ ), an analogous classification of the modes as TE-like and TM-like modes can be made. According to the symmetry planes, TE-like modes (having the fundamental component of the electric field pointing along $y$ ) would be $z$-even modes and the TM-like modes (having the fundamental component of the electric field pointing along $z$ ) would be $z$-odd modes.

As a result, in Fig. 2.1(a), the TE-like modes are presented in blue and the TM-like modes in red for the mirror unit cell used to build our structure. All the photonic band diagram simulations were performed through RSoft Bandsolve [94]. Here, a range of 
(a)

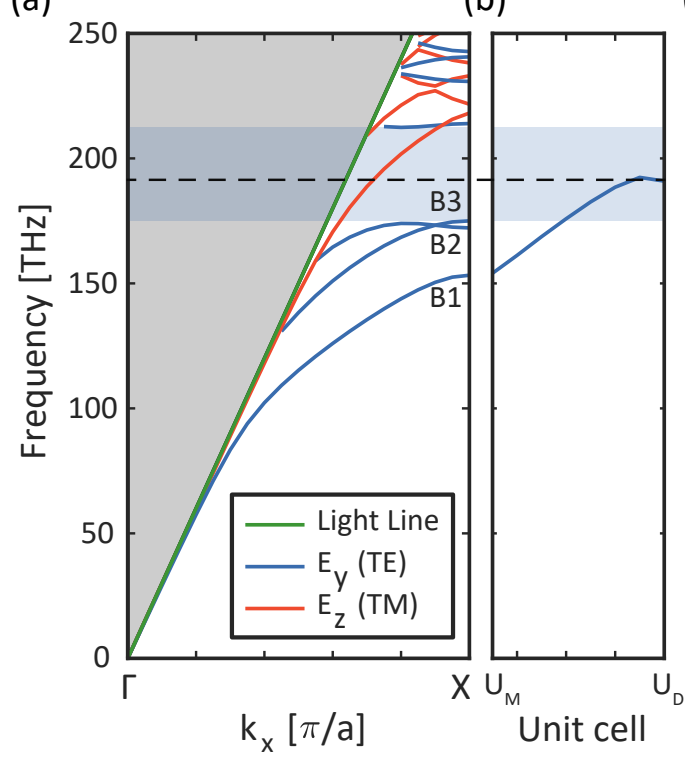

(c)

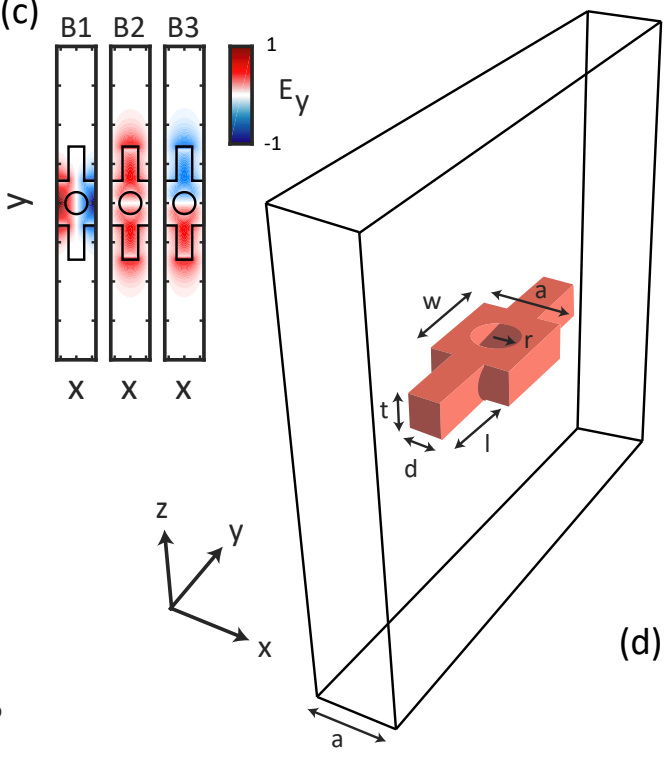

Figure 2.1: (a) Photonic bands for transverse electric (TE-like) modes and transverse magnetic (TM-like) modes for the mirror unit cell. The gray- and light blue-shaded areas denote the non-guided modes and the TE-like quasi-bandgap, respectively. The confined optical mode in the defect zone has been depicted with a dashed line. (b) Evolution of the band B1 from the mirror unit cell $\left(U_{M}\right)$ to the defect unit cell $\left(U_{D}\right)$. (c) Component electric field profile $\left(E_{y}\right)$ of the three first TE-like optical bands at the symmetry point $X$. (d) Supercell used in the simulations of the optical band diagrams showing the silicon unit cell of the OMC surrounded by air.

wave vectors $\mathbf{k}$ along the directions of periodicity $\left(k_{x}\right)$ from the symmetry points $\Gamma$ to $X$ of the irreducible Brillouin zone has been shown [93]. The unit cell has been designed in order to give a TE-like photonic bandgap (depicted with a shaded blue area) where the confined optical cavity mode of the final structure should be placed. This mode has been represented with a black dashed line. The confined mode comes from the evolution of the band named B1 as it evolves for the different unit cells that will constitute the final OM cavity, as can be appreciated in Fig. 2.1(b). Here, we can see the evolution of the band B1 at the symmetry point $X$ in the Brillouin zone from the mirror $\left(U_{M}\right)$ to the defect $\left(U_{D}\right)$ unit cells. The $E_{y}$ field profile of this mode can be seen in the first panel of Fig. 2.1(c), where it can also be found the field profiles for the band modes B2 and B3 for the sake of completeness. For all these calculations, the used unit cell and their labeled parameters are presented in Fig. 2.1(d). It consists of a supercell where the silicon unit cell (in red) is surrounded by a supercell of air. The parameters of the simulated mirror unit cell are $(a, r, d, l)_{M}=(500,150,250,500) \mathrm{nm}$ and the defect ones are $(a, r, d, l)_{D}=(325,98,163,250) \mathrm{nm}$, with $\mathrm{w}=570 \mathrm{~nm}$ and $\mathrm{t}=220 \mathrm{~nm}$ for both of them. The deep and height of the air supercell have been chosen in such a way that interaction between neighborhood supercells is avoided. In this particular case, the deep and height were set as 4 times the lattice period $a$.

Simultaneously to the design of the unit cells from the photonic band diagram, it is necessary to also localize the mechanical mode which will couple to the optical mode. Because of symmetry arguments regarding the final OM coupling [64], a set of mechanical 
modes can be restricted in the analysis. Especially, $X$ symmetry points will result in a vanishing $g_{0}$, thus restricting our analysis to $\Gamma$ symmetry points for the analysis of the phononic band diagrams.

The phononic band diagram is obtained through the analysis of the acoustic master equation of an elastic wave. Considering no body forces as in the optical case [95, 96]:

$$
\rho(\mathbf{r}) \frac{\partial^{2} u_{i}}{\partial t^{2}}-\left[c_{i j k l}(\mathbf{r}) u_{k, l}\right]_{j}=0
$$

where $c_{i j k l}$ is the elastic stiffness or elasticity tensor, $u_{i}$ are the field component of the total displacement profile $\mathbf{u}(\mathbf{r}, t)$ and $\rho(\mathbf{r})$ the scalar density field. Once applying Bloch's theorem, we can solve the previous equation assuming a time harmonic solution, thus resulting in:

$$
\frac{1}{\rho(\mathbf{r})}\left[c_{i j k l}(\mathbf{r}) u_{k, l}\right]_{j}=\Omega^{2} u_{i}
$$

here, $\Omega$ are the resulting obtained mechanical frequencies (eigenvalues) of the involved mechanical mode profiles (eigenvectors).

The resulting evolution of the phononic band diagram is presented in Fig. 2.2(a), from the defect to the mirror unit cell at the $\Gamma$ symmetry point. In Fig. 2.2(b) is presented in more detail all the involved bands from modes of different symmetries for the mirror unit cell. The most important feature to emphasize is the presence of a complete phononic bandgap, where, by proper design, the frequency of the mechanical mode has to be placed. As we noted above, this should reduce the phonon leakage of the final structure as it prevents that the confined mechanical mode couples with modes of different symmetries existing in the mirror regions. These phononic band diagram simulations were performed with COMSOL Multiphysics [97], where a unit cell as the one presented in Fig. 2.2(c) was considered. Here, we set Floquet Periodic Conditions (FPC) at the lateral boundaries of the structure and the remaining boundaries were kept as free. The dimension values of the mirror and the unit cell are the same as the ones used in the photonic band diagram simulations.

It must be pointed out that, in silicon, light and sound have propagation velocities that differ by several orders of magnitude. Thus, the optical and mechanical modes at frequencies $\omega$ and $\Omega$, respectively, will be very different, as well as the mode profiles. As we can see from Fig. 2.1 and 2.2, the confined photonic mode frequency is around 192 $\mathrm{THz}$ and the mechanical one is $3.82 \mathrm{GHz}$.

\subsubsection{Acousto-optic interaction}

Besides propagating independently from each other, the obtained photonic and phononic modes may also interact. This interaction, as it involves both light and sound, is the so-called acousto-optic (AO) - or OM - interaction, which is strongly enhanced when it is confined at the micro- and nano-scale [98].

The classical picture of the AO interaction is that the motion of the mechanical mode can alter the properties of the medium. On the one hand, a traveling wave originates a local stress variation that results in an alteration of the refractive index, phenomenon known as photoelastic (PE) effect. On the other hand, this acoustic wave can cause any material interface to vibrate, thus resulting in a change in the boundary conditions of the system (a deformation) and thus producing a variation in the dielectric permittivity. 
(a)

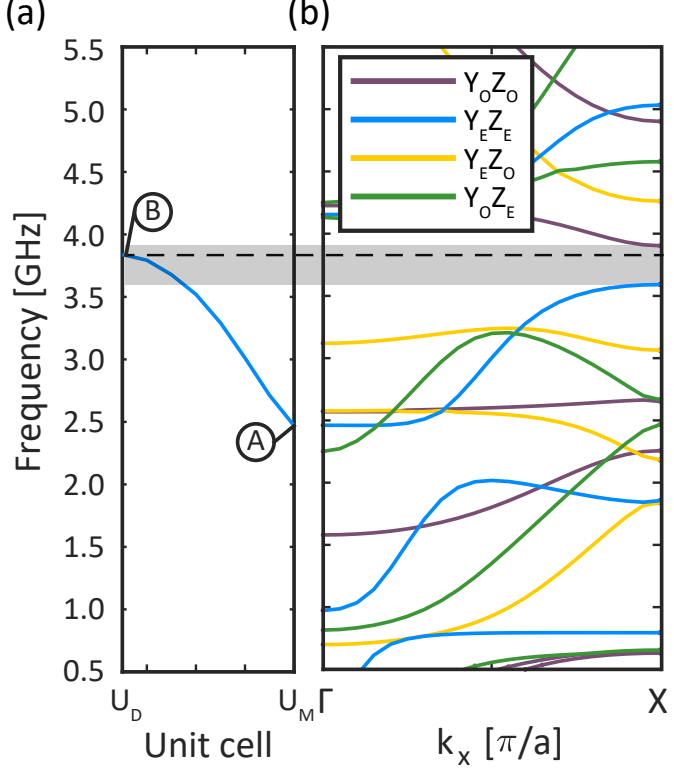

(c)

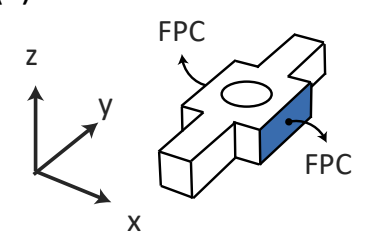

(d)

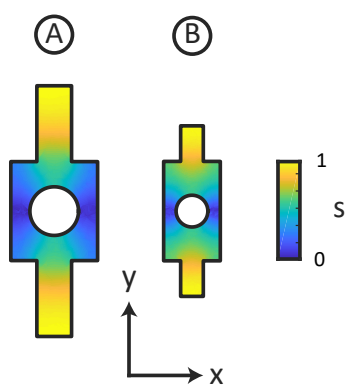

Figure 2.2: (a) Phononic band diagram evolution of the confined mechanical mode band from the defect $\left(U_{M}\right)$ to the mirror $\left(U_{D}\right)$ unit cell. (b) Phononic bands for mechanical modes with (odd, odd) in purple, (even, even) in blue, (even, odd) in yellow and (odd, even) in green symmetries respect to the $\mathrm{Y}$ and $\mathrm{Z}$ axis planes. The gray-shaded area denotes the total phononic bandgap and the confined mechanical mode in the defect zone has been depicted with a dashed line. (c) Mechanical unit cell showing the boundary Floquet Periodic Condition (FPC) of the simulation. (d) Mechanical field profile of $\left(U_{M}\right)$ in $\mathrm{A}$ and $\left(U_{D}\right)$ in $\mathrm{B}$.
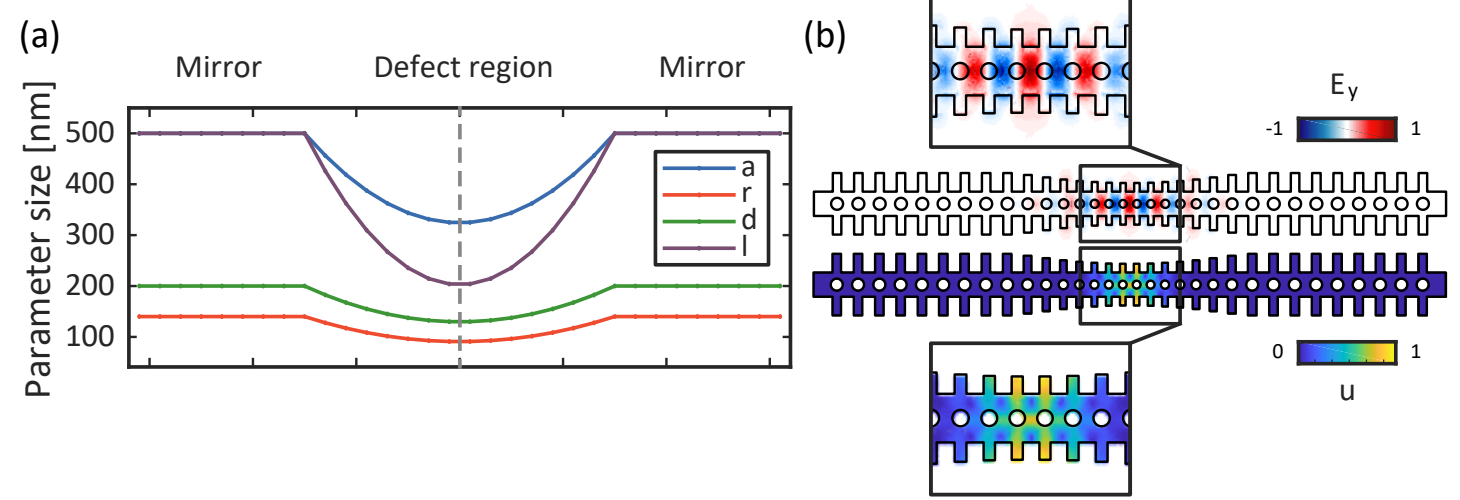

Figure 2.3: (a) Parameter variation to build the OM cavity for the nominal structure. (b) $E_{y}$ profile of the optical resonance at $\lambda_{c}=1530 \mathrm{~nm}$ and total mechanical displacement profile at $\Omega_{m} / 2 \pi=3.82 \mathrm{GHz}$. 
This last effect is known as the moving interface (MI) effect and, in our case, it will correspond to the movement of the silicon boundaries of the cavity surrounded by air.

In order to calculate the strength of the $\mathrm{AO}$ interaction, the $\mathrm{OM}$ coupling rate $g$ is introduced, which accounts for how much the optical mode is shifted by unit of displacement [2]. This interaction strength was also described in Section 1.2.1. Besides the PE effect and the MI effect giving rise to frequency shifts, there are also other effects than can contribute as those related to thermorefractive effects or surface effect [64]. However, the last ones are assumed to be negligible and the total OM coupling rate results in:

$$
g=g_{M I}+g_{P E}
$$

The calculation of the MI coupling coefficient is performed using the derivation by Johnson et al. in Ref. [99]. In our case, we were considering a silicon cavity surrounded by air, thus resulting in a $g_{M I}$ equal to:

$$
g_{M I}=-\frac{\omega}{2} \frac{\oint(\mathbf{U} \cdot \hat{\mathbf{n}})\left(\Delta \varepsilon\left|\mathbf{E}_{\|}\right|^{2}-\Delta \varepsilon^{-1}\left|\mathbf{D}_{\perp}\right|^{2} d S\right)}{\int \mathbf{E} \cdot \mathbf{D} d V} \sqrt{\frac{\hbar}{2 m_{e f f} \Omega}}
$$

where $\mathbf{U}$ is the normalized displacement field $(\max |\mathbf{U}|=1), \hat{\mathbf{n}}$ is the outward pointing normal to the boundary, $\Delta \varepsilon=\varepsilon_{S i}-\varepsilon_{A i r}$ and $\Delta \varepsilon^{-1}=\varepsilon_{S i}^{-1}-\varepsilon_{A i r}^{-1}$. From our notation, $\omega$ and $\Omega$ are the optical and acoustic frequencies and $m_{\text {eff }}$ is the effective motional mass related with the normalized acoustic displacement by $m_{e f f}=\rho \int|\mathbf{U}|^{2} d V$ [92].

Concerning the PE coupling rate contribution, it can be calculated as [64]:

$$
g_{P E}=-\frac{\omega}{2} \frac{\langle E|\delta \varepsilon| E\rangle}{\int \mathbf{E} \cdot \mathbf{D} d V} \sqrt{\frac{\hbar}{2 m_{e f f} \Omega}}
$$

with $\delta \varepsilon=-\varepsilon_{0} n^{4} p_{i j k l} S_{k l}$. Here, $\varepsilon_{0}$ is the vacuum permittivity, $n$ the refractive index of silicon, $p_{i j k l}$ are the photoelastic tensor components and $S_{k l}$ the strain tensor components.

With regard to our system, through a quadratic adiabatic transition between the mirror unit cell to the defect unit cell, the resulting OM crystal cavity is created. The parameter variation to built the cavity is presented in Fig. 2.3(a) for the main parameters described in Fig. 2.1(d). The resulting optical and mechanical modes are shown in Fig. 2.3(b). These photonic and phononic mode profiles overlap in the middle region of the cavity, as can be seen in the insets panels in Fig. 2.3(b). The calculated OM coupling rate, henceforth simplified as $g_{0}$, for our configuration was estimated to be $g_{0} / 2 \pi \simeq 540 \mathrm{kHz}$.

Here, we have studied the AO interaction resulting from the propagation of an acoustic wave. Similarly, optical waves cause volume optical forces that induce a strain field through electrostriction (ES), the complementary situation of the PE effect. These forces also can produce an effect on the boundaries, as the radiation pressure (RP) effect. These ES and RP effects will be broadly discussed in Chapter 6 .

\subsubsection{Fabricated OM cavity}

Once we have our theoretical designed structure, the 1D OM crystal cavities were fabricated on standard SOI wafers with a top silicon layer thickness of $220 \mathrm{~nm}$ and a buried oxide layer thickness of $2 \mu \mathrm{m}$. The patterned structure was written by electron beam 
lithography, performed with a Raith150 tool, in a PMMA positive resist film and transferred into the silicon layer by reactive ion etching. Once the silicon was etched, a buffered hydrofluoric acid was applied to remove the buried oxide layer to release the structured nano-beams [31]. A clean procedure with a Tepla Plasma System 200 is also performed in case of dust deposition. The parameters of the cleaning process are power $400 \mathrm{~W}$, pressure 1.5-1.6 mBar and oxygen $\left(O_{2}\right)$ flow $100 \mathrm{sccm}$, all along 10 minutes. For more details regarding the fabrication process, which was applied to all the samples characterized during the Thesis, see Appendix A.

(a)

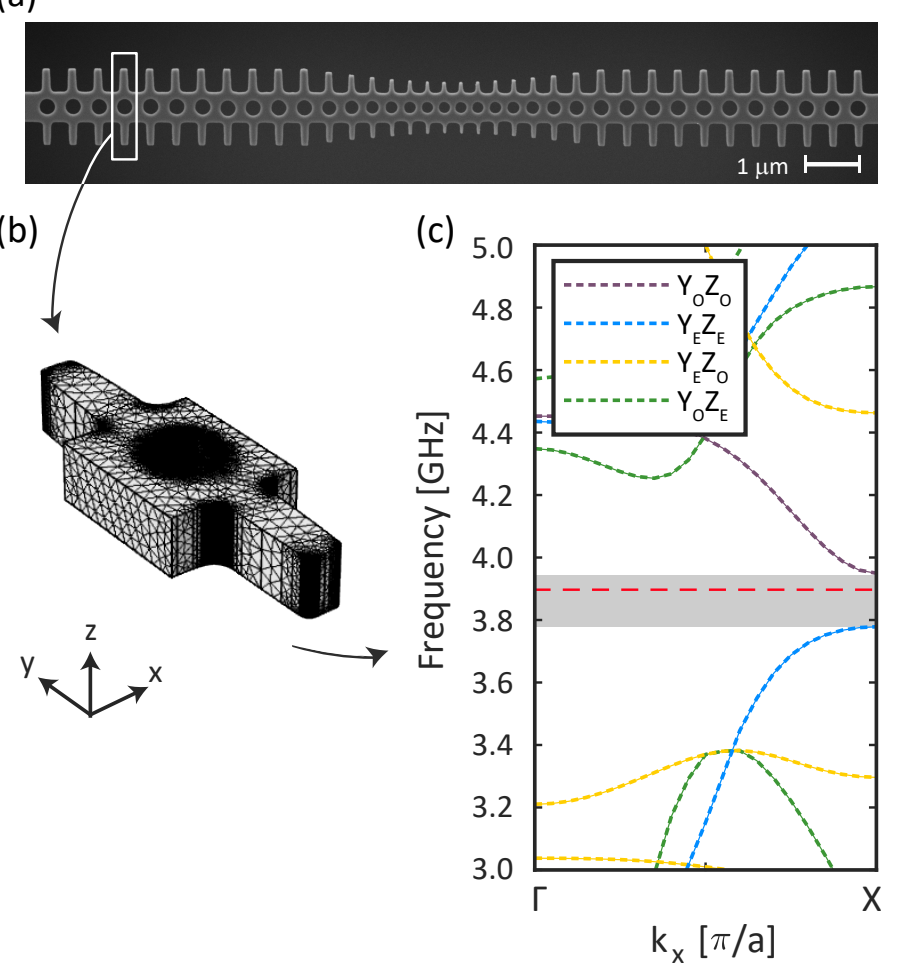

(d)
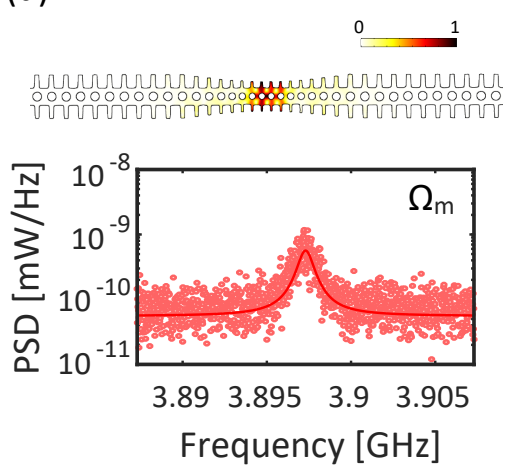

(e)

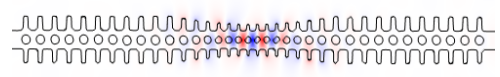

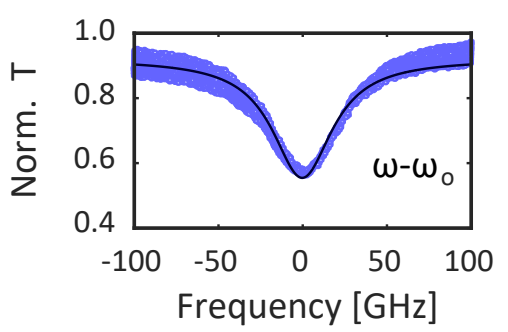

Figure 2.4: (a) SEM image showing the OM crystal cavity used in the experiments with a framed mirror unit cell. (b) Retrieved mesh profile from a SEM image of the mirror unit cell. (c) Phononic band diagram obtained from the averaged calculations of different retrieved SEM mirror cells. (d) SEM retrieved phononic profile and experimental PSD from the OMC. (e) SEM retrieved photonic profile and experimental optical response which has been centered with respect to the middle optical resonance of the OMC.

Following this fabrication procedure, a set of $\mathrm{OM}$ cavities were fabricated according to the design summarized in Fig. 2.3. However, because of fabrication imperfections (rounding of the corners, sidewall roughness), the fabricated structures resulted different from the designed nominal ones. Regarding the mechanical properties, fabrication imperfections may eventually lead to structures in which the mechanical breathing mode is no longer confined within the mechanical bandgap. To determine whether the fabricated structures also displayed a full phononic bandgap, we simulated numerically an OM cavity using the exact dimensions retrieved from scanning electron microscope (SEM) images. A SEM image of our fabricated structure is presented in Fig. 2.4(a), where the 
white rectangle points, as an example, a single unit cell where the retrieved dimension analysis has been performed. This procedure was carried out by using a software that enabled us to convert the SEM image into a COMSOL schematic, thus resulting in an unit cell as the one shown in Fig. 2.4(b). This software consisted in a image editor (by means of Photoshop, Illustrator or even Matlab) used to get only the cavity profile and to convert the pixel-based image in a vector graphics file ready to be open by COMSOL. By means of this procedure, we simulated different unit cells in the mirror region of the cavity, which resulted in the final phononic band diagram in Fig 2.4(c). It can be seen that the full mechanical bandgap remains in the fabricated sample. Here, the dashed line corresponds to the experimentally confined mechanical mode, whose power spectral density (PSD) measurement is shown in Fig. 2.4(d) with its real mechanical mode profile. This profile was also retrieved from the SEM image. Additionally, we also simulated numerically the optical profile of the fabricated sample, presented in the top inset of Fig. 2.4(e) with an intrinsic optical quality factor $Q_{i}=5.9 \times 10^{4}$. An example of the experimental optical response resulting from the characterization of this cavity is also shown in Fig. 2.4(e). The experimental setups used to measured all the experimental parameters given along this thesis are summarized and discussed in the last section of each corresponding chapter.

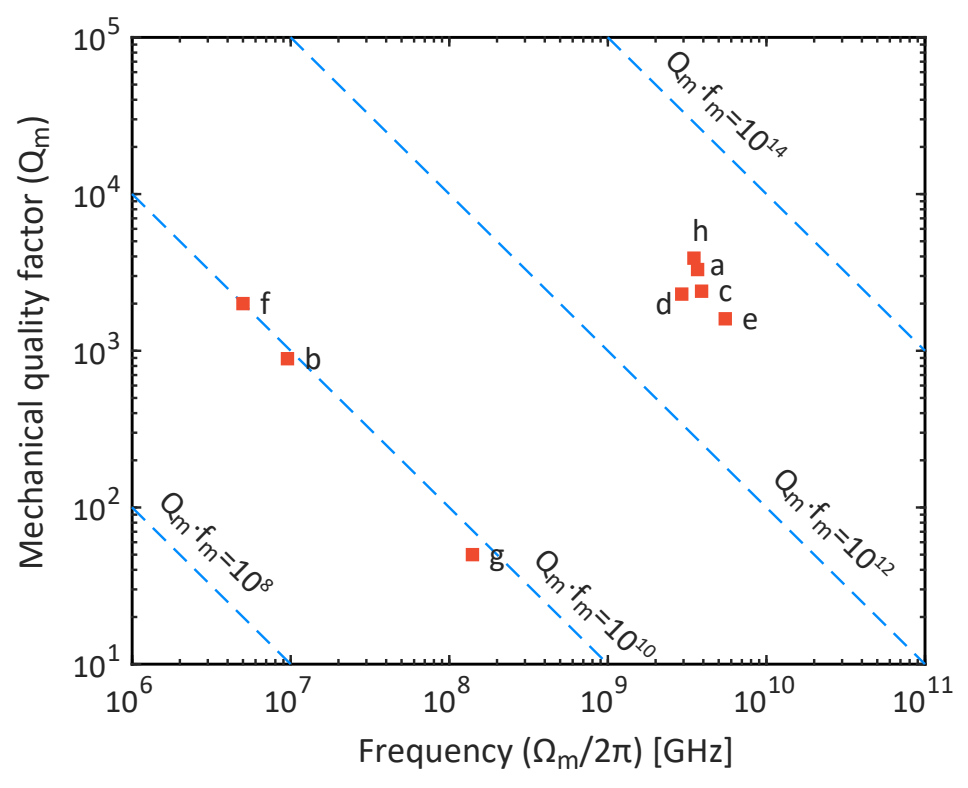

Figure 2.5: Mechanical quality factor vs. the mechanical frequency for published OM crystal cavities at room temperature at a - [64], b - [100], c -(This work) [101], d - [44], e - [67], f - [102], g - [103] and h - [104].

The experimental measurements were performed at room temperature and atmospheric pressure by coupling the light into and out of the cavity with a micro-loop fiber taper (see Section 2.5.1 for more experimental details). Regarding the optical properties, the tested unheated OM cavity had an optical resonance at $\lambda_{r}=(1522.5 \pm 0.3)$ $\mathrm{nm}$ showing a loaded optical quality factor of $Q_{o}=5 \times 10^{3}$ and an overall decay rate of $\kappa / 2 \pi=39 \mathrm{GHz}$. This last parameter accounts for the total cavity loss rate which can be derived as the sum of the contributions of the loss rate of the input coupling $\left(\kappa_{e x}\right)$ and the loss remaining internal losses $\left(\kappa_{0}\right)$. The experimentally transduced me- 
chanical mode of the cavity is shown in Fig. 2.4(d) and shows a resonance frequency at $f_{m}=\Omega_{m} / 2 \pi=3.897 \mathrm{GHz}$ with a mechanical quality factor of $Q_{m}=\Omega_{m} / \Gamma_{m}=(2400 \pm 300)$, which gives a $Q_{m} \times f_{m}$ value of $(9.5 \pm 1.1) \times 10^{12}$. This value is in accordance with other OM crystal cavities measured at room temperature, as can be seen in the overview plot of the state of the art $Q_{m}$ versus $f_{m}$ of this sort of cavities. Here, $\Gamma_{m}$ is the measured mechanical linewidth $\Gamma_{m} / 2 \pi=(1.6 \pm 0.2) \mathrm{MHz}$. The experimental measurements, as mentioned above, as broadly discussed and described in the experimental setups section (Sec. 2.5.1 and Sec. 2.5.2). In accordance with the aforesaid discussion, from Fig. $2.4(\mathrm{c})$ it can be seen that the confined mechanical mode (red-dashed line) lies inside the total phononic bandgap. Therefore, we can state that this OM crystal cavity possesses a breathing mode within a mechanical bandgap with a measured $g_{0} / 2 \pi=(660 \pm 70) \mathrm{kHz}$, which is of the same order of magnitude as the value calculated for the nominal cavity. Regarding the OM cooperativity, another key parameter in cavity optomechanics that is defined as $C_{0}=\left(4 g_{0}^{2}\right) /\left(\Gamma_{m} \kappa\right)$, we get a value of $C_{0}=(2.8 \pm 0.5) \times 10^{-5}$.

Concerning the obtained mechanical oscillator parameters, it may be of interest to compare values as the mechanical quality factor with other reported structures under the same conditions. Then, a plot showing the mechanical quality factor against the mechanical frequency is presented in Fig. 2.5. This representation provides information about the $Q_{m} \times f_{m}$ rate of our oscillator in comparison with other OM cavities at room temperature. We can see that our system performs in close vicinity of the best OM cavities reported in the literature. The labeled points correspond to different works, corresponding to a - [64], b - [100], c -(This work) [101], d - [44], e - [67], f - [102], g - [103] and h - [104].

\subsection{Optomechanical microwave oscillator}

High-quality microwave sources with ultranarrow linewidths are required for a great variety of applications. Typically, microwave oscillators are made by applying frequency multiplication to an electronic source. This requires a cascade of frequency-doubling stages, which means that the power of the final signal is greatly reduced. Recently, different techniques to produce microwave tones via optical means have been proposed. The resulting device is called an optoelectronic oscillator (OEO) [105, 106].

Amongst the different techniques to build an OEO, Stimulated Brillouin Scattering (SBS) has proven itself to be extremely useful since it can provide high-frequency RF signals with extreme purity (or extremely narrow linewidth). For instance, cascaded Brillouin scattering on a high-Q silica wedge cavity enabled the synthesis of a $21 \mathrm{GHz}$ microwave tone with a record-low phase noise floor value of $-160 \mathrm{dBc} / \mathrm{Hz}$ [107]. Owing to the equivalence between Brillouin scattering in a waveguide and $\mathrm{OM}$ interaction in a cavity [62], we could think that an OEO can be also obtained from an OM cavity when pumped with a blue-detuned laser source. In this case, since the involved mechanism is a self-sustained oscillation originated from OM interaction, we may call the resulting device an optomechanical oscillator (OMO).

In an OM crystal cavity, blue-detuned driving gives rise to a change of the mechanical frequency (the so-called optical spring effect) as well as a reduction of the overall mechanical damping rate $\Gamma_{\text {eff }}=\Gamma_{m}+\Gamma_{\text {opt }}$ [2]. In our case $\left(\Gamma_{m} \ll g \ll \kappa\right)$ the 
optomechanically-induced damping rate can be calculated as [108]:

$$
\Gamma_{e f f}=\Gamma_{m}+\Gamma_{o p t}=\Gamma_{m}+g^{2} \frac{\Omega_{m}}{\omega}\left[\frac{\kappa}{(\Delta+\omega)^{2}+(\kappa / 2)^{2}}-\frac{\kappa}{(\Delta-\omega)^{2}+(\kappa / 2)^{2}}\right]
$$

where $\Delta$ is the laser detuning given by $\Delta=\omega_{L}-\omega_{c}$ with $\omega_{i}=c / \lambda_{i} . g$ is the lightenhanced OM coupling strength which is related with the vacuum OM coupling rate as $g=g_{0}{\sqrt{n_{c a v}}}_{\text {. }}$. Here, $\bar{n}_{\text {cav }}$ is the steady-state cavity population or photon number circulating inside the cavity given by [2]:

$$
\bar{n}_{c a v}=\frac{\kappa_{e x}}{\Delta^{2}+(\kappa / 2)^{2}} \frac{P_{i n}}{\hbar \omega_{L}}
$$

with $P_{\text {in }}$ as the input power.
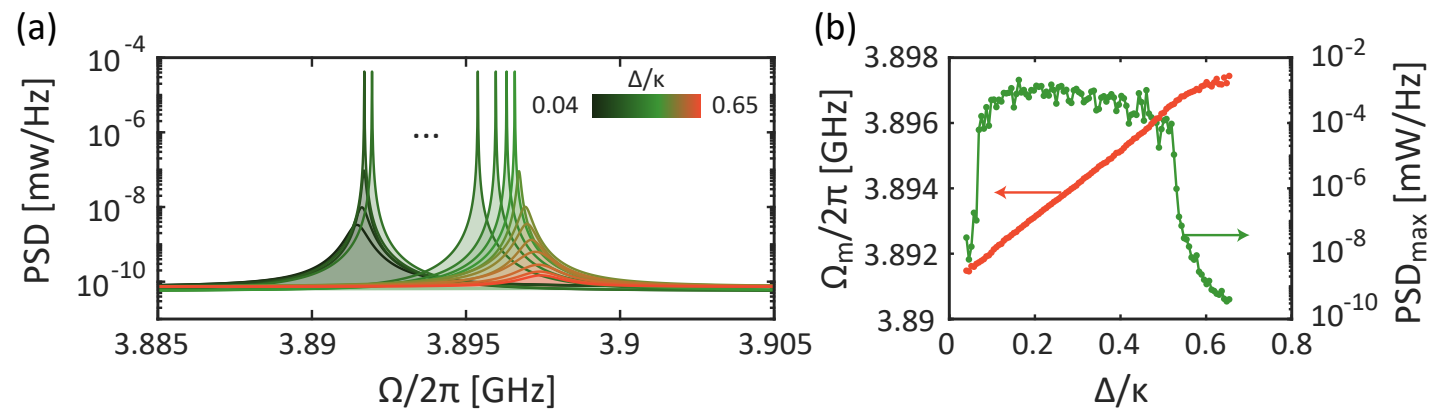

Figure 2.6: (a) PSD evolution of the confined mechanical mode in the blue detuned regime. (b) Maximum PSD amplitude (green) and center mechanical frequency (red) evolution as a function of the normalized relative laser detuning $(\Delta / \kappa)$.

According to this, the evolution of the mechanical motion of the cavity and, therefore, its damping rate, can be studied by photo-detecting the signal scattered from the cavity under blue-detuned driving conditions, as shown in Fig. 2.6(a). These measurements - taken in reflection after photodetection of the signal backscattered by the OM cavity - were recorded when the thermo-optic effect shifts the optical resonance up to higher wavelengths in a typical bistability "saw-tooth" shaped transmission [67], which required a threshold power $P_{\text {in }}=2.48 \mathrm{~mW}$ reaching the fiber loop which couples to the cavity. The large pump threshold is probably due to the fact that the optical quality factor of the cavity is relatively low so the device is operated in the unresolved sideband regime $\left(\kappa \gg \Omega_{m}\right)$. Increasing the optical quality factor of the cavity should reduce this pump threshold, because, in this regime, for the optimal detuning, when $\Delta=\frac{\kappa}{2}, \Gamma_{o p t}=-8\left(\frac{g}{\kappa}\right)^{2}$.

From Fig. 2.6(a), it can be seen that for higher laser detunings in the blue-detuned regime $(\Delta>0)$ the mechanical mode is both transduced and amplified. However, a threshold of instability can be attained once the mechanical losses equate to the optical antidamping when $\Gamma_{e f f}=0$. Thus, the oscillator reaches the condition of self-oscillation or phonon lasing, which results in a very narrow tone in the detected spectrum at the mechanical resonance $[67,109]$. This can be seen in Fig. 2.6(a) around $\Delta / \kappa=0.53$ in our case. These measurements were performed at an input power of $P_{i n}=2.48 \mathrm{~mW}$. Once this regime is reached, it can be observed that it remains along with a different set of wavelengths providing a mechanical frequency tunability of the oscillator of the order 
of $4 \mathrm{MHz}$. However, after reaching the so-called single self-oscillation (SSO) regime (we used this term to differentiate this regime from the case of multiple oscillations, as it will be seen in detail below), the mechanical mode is slightly cooled just before it exits the resonance. The evolution of center mechanical frequency and the amplitude of the PSD is depicted in Fig. 2.6(b).

It has to be noted that the mechanical modes presented in Fig. 2.6(a) are the resulting peak fits of the transduced motion. For the overall amplified peaks, the fit is performed through a Lorentz's fit as presented in Fig. 2.4(d). However, once the SSO is attained, as the theoretical $\Gamma_{e f f}=0$, the measured linewidth will become very small, being ultimately limited by the resolution bandwidth of the employed spectrum analyzer. For example, in Fig. 2.7(a) is presented the measured PSD (light red dots) and its Voigt fit (red line). A close view of the central peak can be seen in Fig. 2.7(b), for a resolution bandwidth (RBW) of $1196 \mathrm{~Hz}$. Thus, the resulting spectrum is the convolution of the Lorentz shape peak from the self-oscillating mode and a Gaussian filter performed by the spectrum analyzer. This convolution between a Lorentzian line shape $(L(\Omega ; \Gamma))$ and a Gaussian broadening function $G(\Omega ; \sigma)$ is the so-called Voigt function, which can be read as:

$$
V(\Omega ; \sigma, \Gamma)=\int_{-\infty}^{\infty} G\left(\Omega^{\prime} ; \sigma\right) L\left(\Omega-\Omega^{\prime} ; \Gamma\right) d \Omega^{\prime}
$$

where $\Gamma$ and $\sigma$ are the Lorentzian and Gaussian linewidths, respectively.

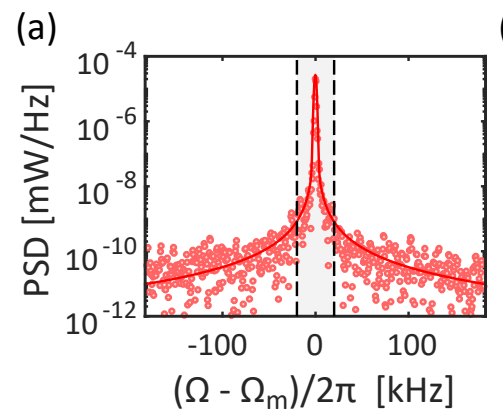

(b)

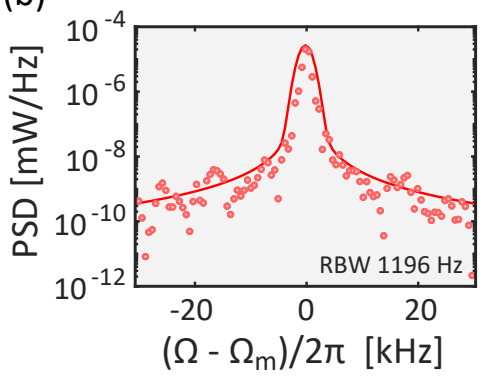

(c)

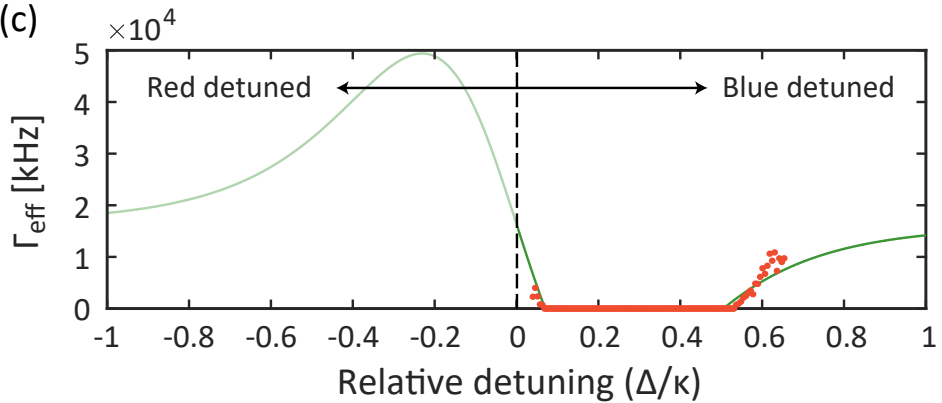

(d)

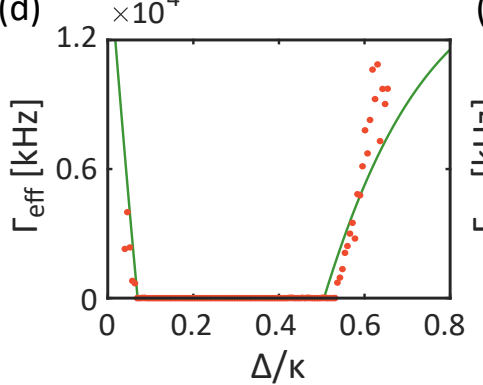

(e)

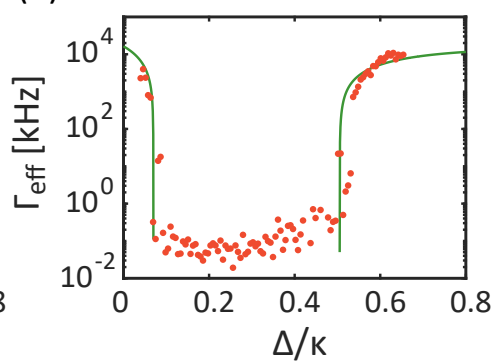

Figure 2.7: (a) Centered measured PSD spectrum of the mechanical mode in the phonon lasing state (red light dots) and Voigt fit (red line). (b) Close view of the Voigt shape fit with a RBW of $1196 \mathrm{~Hz}$. (c) Evolution experimental (red light dots) and expected (green line) overall damping rate as a function of the normalized relative detuning. (d) Close view of the blue-detuned evolution of the overall damping rate in linear scale. (e) Close view of the blue-detuned evolution of the overall damping rate in logarithmic scale.

Taking into account the last considerations about both the Lorentzian and Voigt 
fits, the evolution of the overall mechanical damping rate (light red dots) is presented in Fig. 2.7(c). In this figure, we have also depicted the evolution of the expected $\Gamma_{\text {eff }}$ following Eq. 2.8, showing a good agreement. It must be noted that as we perform the experiments in the blue detuned regime, only $\Delta / \kappa>0$ are shown. A close view in both linear and logarithmic scale are shown in Fig. 2.7(d) and (e), respectively.

\subsubsection{OMO phase noise}

A key feature of microwave oscillators is the characterization of random fluctuations in the phase of the signal under study. In the frequency-domain representation, these fluctuations can be measured by the so-called phase noise, which is related to the time domain deviations from the case of an ideal signal with zero jitter.

In our case, once the mechanical mode is in the self-sustained oscillation mode or lasing regime, the generated microwave tone can be also characterized by these means. In such a way, we measured the phase noise of the generated microwave tone at $\Omega_{m} / 2 \pi=$ 3.895 GHz, as shown in Fig. 2.8(a). The phase noise shows the typical dependencies with $1 / f^{4}$ (random walk of frequency, the lower part of the spectrum), $1 / f^{3}$ (flicker frequency noise), $1 / f^{2}$ (white frequency noise or random walk) and $1 / f^{0}$ (white phase noise), which are in good agreement with the general phase noise $\mathcal{L}(f)$ described by the Leeson's model [110-112].

To define the power-law function used to describe the Lesson's model, we first introduce another frequently-used parameter to measure the phase noise: the one-sided power spectral density of the random fluctuation, $S_{\varphi}(f)$, which is presented in Fig. 2.8(b). It is related with the single-sideband noise spectrum $\mathscr{L}(f)$ given by:

$$
\mathscr{L}(f)=\frac{1}{2} S_{\varphi}(f)
$$

Note that this is the linear single sideband phase noise, which is related with the logarithmic single sideband phase noise scale as $\mathscr{L}(f)=10^{\mathscr{L}_{d B c}(f) / 10}$. Moreover, as the oscillator noise can also be related with the frequency fluctuation, instead of the phase fluctuation, it is worth analyzing the parameter $S_{y}(f)$ (Fig. 2.8(c)) as:

$$
S_{y}(f)=\frac{f^{2}}{\nu_{0}^{2}} S_{\varphi}(f)
$$

where $\nu_{0}$ is the carrier frequency of the reference oscillator.

Therefore, the power law function can be described as:

$$
S_{\varphi}(f)=\sum_{-4}^{0} b_{i} f^{i}
$$

where $b_{i}$ are coefficients of each one of the phase noise contributions. These coefficients can be identified as the intercepts of the straights lines with slope $i$, once we plot the power laws in a log-log scale as in Fig. 2.8 [112].

Regarding the phase noise, it becomes as low as $(-100 \pm 1) \mathrm{dBc} / \mathrm{Hz}$ at $100 \mathrm{kHz}$, which is a remarkably good value for a nanoscale $\mathrm{GHz}$ microwave oscillator. Noticeably, this performance is on par with some commercial mid-range devices, such as the Agilent N5183AMXG that displays a phase noise of $-102 \mathrm{dBc} / \mathrm{Hz}$ at $100 \mathrm{kHz}$ offset for a 10 $\mathrm{GHz}$ frequency [113]. Nevertheless, in order to establish a fair comparison with other OMOs, we have calculated the equivalent phase noise at $100 \mathrm{kHz}$ for a $5 \mathrm{GHz}$ carrier 


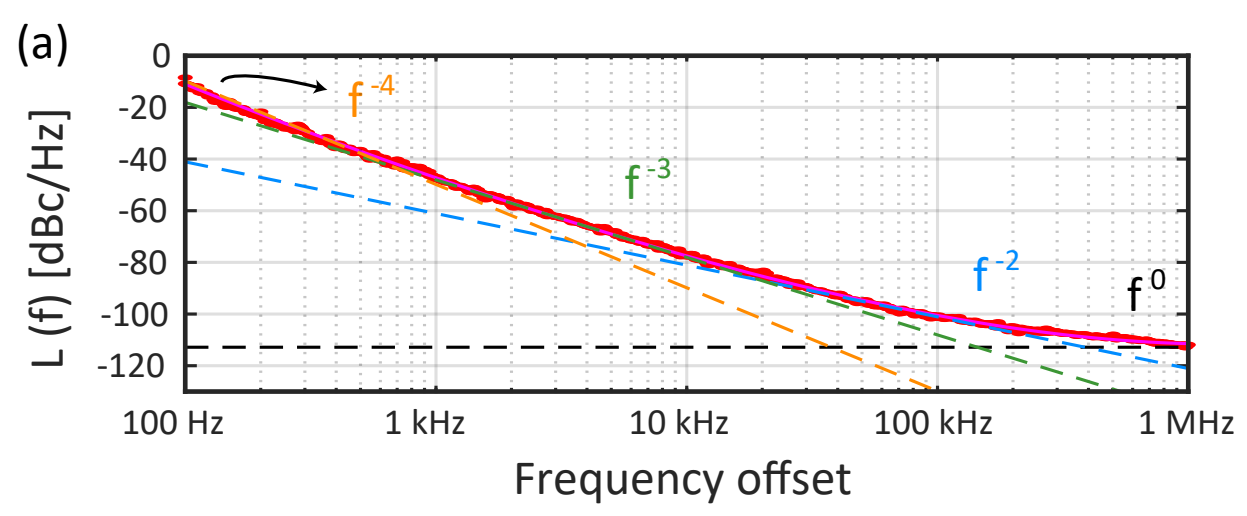

(b) $\quad$ (c)

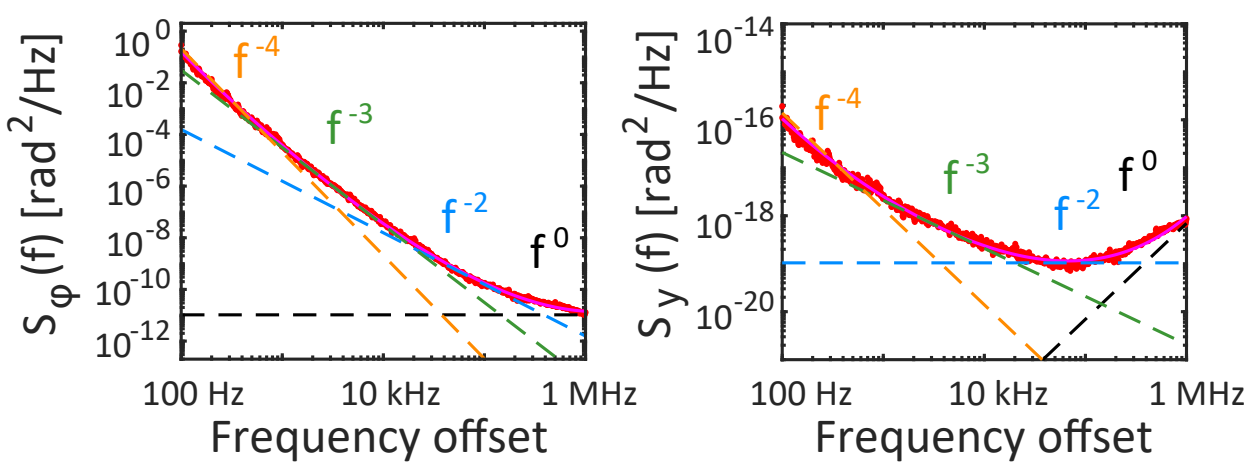

Figure 2.8: (a) Phase noise of the generated microwave tone in red. (b) One-sided power spectral density of the random phase fluctuation (red curve). (c) Frequency noise spectra (red curve). In all the panels, the phase noise fit contributions as described by the Leeson's model are depicted by dashed lines.

frequency using the transformation $P N_{1}=P N_{0}+20 \cdot \log \left(f_{1} / f_{0}\right)$. Here, $P N_{0}$ is the phase noise value at frequency $f_{0}$ of the structures to be compared and $f_{1}$ is the new equivalent carrier frequency which we have chosen to be $5 \mathrm{GHz}$. This way, we can easily compare the performance of oscillators operating at frequencies that can differ by orders of magnitude. The results of equivalent phase noise $P N_{1}$ are summarized in Table 2.1. Importantly, our device improves the noise performance of silicon disks fabricated in MEMS technology [114]. The OMO reported in [44], which makes use of a 1D OM crystal cavity in GaAs, shows a phase noise at $100 \mathrm{kHz}$ more than $10 \mathrm{~dB}$ worse than our device for a similar microwave frequency. Since both OM cavities display similar mechanical $\mathrm{Q}$ factors at room temperature (limited by material losses), the better performance of our device in terms of phase noise may be due to an improved value of $g_{0}$ or a higher driving power. On the other hand, Brillouin-based OMOs show a better performance than OM crystal cavities (phase noise around $-110 \mathrm{dBc} / \mathrm{Hz}$ at $f_{R F}=21.7 \mathrm{GHz}$ ) as a result of the ultrahigh optical quality factor but at the expenses of a larger cavity footprint $\left(>>\mathrm{mm}^{2}\right)$ [107]. Therefore, with the advantages of extreme compactness and silicon-technology compatibility, our approach is a very promising candidate to build ultracompact OMOs on silicon chips. 
Table 2.1: Comparison of the phase noise of different OMOs.

\begin{tabular}{cccccc}
\hline Reference & Structure & $f_{R F}(\mathrm{GHz})$ & $\begin{array}{c}\mathrm{PN} \text { at 100 kHz } \\
\text { (in dBc/Hz) }\end{array}$ & $\begin{array}{c}\text { Foot-print } \\
\text { Equivalent PN } \\
\text { at 5 GHz }\end{array}$ \\
\hline$[111]$ & SiN ring resonator & 0.042 & -120 & $\approx 1 \mathrm{~mm}^{2}$ & -78 \\
{$[115]$} & 2 Coupled ring MEMS & 0.176 & -112 & $>10 \mu \mathrm{m}^{2}$ & -83 \\
{$[44]$} & 1D III/V OM photonic-crystal cavity & 2.92 & -87 & $<10 \mu \mathrm{m}^{2}$ & -83 \\
{$[116]$} & Integrated OEO loop circuit & 8.87 & -80 & $\approx 30 \mathrm{~cm}^{2}$ & -84 \\
{$[117]$} & Si 2D photonic crystal cavity & 0.112 & -125 & $<10 \mu \mathrm{m}^{2}$ & -92 \\
{$[114]$} & MEMS oscillator & 1.12 & -107 & $>>\mathrm{um}^{2}$ & -94 \\
This work & 1D Si OM photonic-crystal cavity & 3.87 & -100 & $<10 \mu \mathrm{m}^{2}$ & -97 \\
{$[118]$} & Film bulk acoustic resonator & 2 & -120 & $>>\mathrm{um}^{2}$ & -112 \\
{$[107]$} & Silica disk & 21.7 & -110 & $>>\mathrm{mm}^{2}$ & -122 \\
\hline
\end{tabular}

\subsubsection{RMS Jitter}

Besides analyzing the phase noise as a measure of the stability of a signal, we can also study the root mean square (RMS) jitter $\left(J_{R M S}\right)$. Specifically, the phase noise describes the instability of the frequency of the studied oscillator expressed in the frequency domain, meanwhile, the jitter is a fluctuation of the signal waveform in the time domain [119]. The RMS jitter can be obtained by integrating the phase noise power data. Note that it is calculated over a specific frequency range by performing the integral value of a set of offset frequencies as [117]:

$$
J_{R M S}=\frac{1}{2 \pi \nu_{0}} \sqrt{2 \int_{f_{1}}^{f_{2}} 10^{\mathscr{L}(f) / 10} d f}
$$

where $f_{1}$ and $f_{2}$ are the start and stop frequency, respectively.

The obtained RMS timing jitter, in comparison with other OMCs, is presented in Table 2.2. The RMS jitter values of [120] correspond to the case of a free-running OMO, for direct comparison. So, our system has competitive values regarding the RMS jitter in comparison with other reported OMOs but it has the advantage of operating at higher frequencies (in the relevant $\mathrm{GHz}$ regime). Here, it has to be noted that our system is a free-running oscillator. However, it has been demonstrated that locking the OMO by an external signal highly reduces the timing jitter [120], a result that could also be used in our structure.

Table 2.2: Comparison of the RMS jitter of different OMOs.

\begin{tabular}{cccccc}
\hline Reference & $f_{R F}(\mathrm{GHz})$ & $J_{R M S}(\mathrm{ps})$ & $J_{R M S}(\mathrm{ps})$ & $J_{R M S}(\mathrm{ps})$ & $J_{R M S}(\mathrm{ps})$ \\
& & $100 \mathrm{~Hz}-1 \mathrm{kHz}$ & $1 \mathrm{kHz}-10 \mathrm{kHz}$ & $10 \mathrm{kHz}-100 \mathrm{kHz}$ & $100 \mathrm{~Hz}-1 \mathrm{MHz}$ \\
\hline$[120]$ & 0.065 & & 210.45 & 36.68 & - \\
This work & 3.87 & 92.3 & 5.3 & 0.63 & 92.4 \\
{$[117]$} & 0.112 & - & - & - & 10.1 \\
\hline
\end{tabular}

\subsubsection{Allan deviation}

Another metric to characterize the frequency stability of microwave oscillators is the Allan deviation $\left(\sigma_{y}(\tau)\right)$ or Allan variance $\left(\sigma_{y}^{2}(\tau)\right)$. Here, as the Allan variance is a 
measurement of the frequency stability, it can be derived from the phase noise measurements [112]:

$$
\sigma_{y}^{2}(\tau)=2 \int_{0}^{\infty} S_{y}(f) \frac{\sin ^{4}(\pi \tau f)}{(\pi \tau f)^{2}} d f
$$

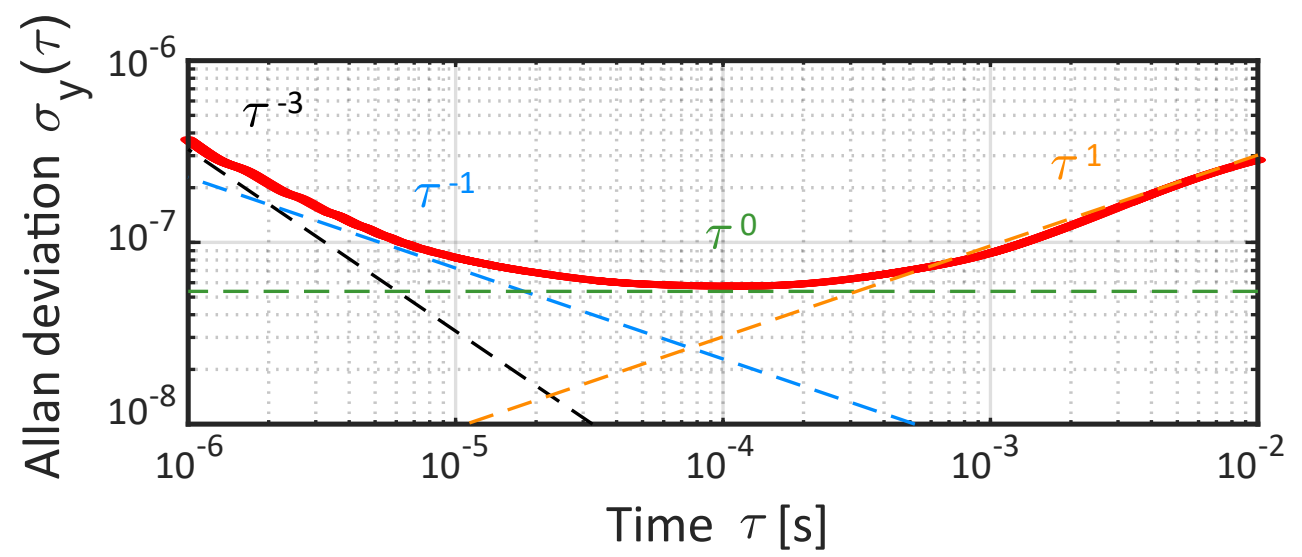

Figure 2.9: Corresponding Allan deviation results converted from the measured phase noise results for the OMO under study (in red) and its power law fits contributions (dashed lines).

Noticeably, despite the conversions between $\mathscr{L}(f), S_{\varphi}(f)$ and $S_{y}(f)$ do not lose information, this is not the case of the conversion from the phase noise spectra to the Allan deviation. This conversion is always approximate because the integral in Eq. 2.15 has to be calculated over the measurement frequency offset set, besides other errors related with the transfer functions [112]. Nevertheless, the Allan deviation shown in Fig. 2.9 results in a satisfactory approximation because we can see a good agreement between the converted Allan deviation through Eq. 2.15 (in red) and the calculated power-law fits (in dashed lines) obtained in Fig. 2.8 through the required conversion relationships [112].

\section{$2.4 \mathrm{OM}$ frequency comb generation}

As shown in the previous section, once the cavity enters into the lasing regime, the system behaves as an OMO. However, higher-order harmonics can also be observed in the detected signal. The underlying process is similar to cascaded Brillouin scattering in an optical waveguide [121]: the Stokes and anti-Stokes photons (resulting from photon-phonon scattering from the laser source) become new pump signals that generate new Stokes and anti-Stokes photons via photon-phonon interaction, and the process is repeated over and over as long as the resulting photons can propagate in the medium (Fig. 2.10(a)). This process can become extremely efficient in an optical cavity supporting both optical and mechanical. For instance, it can happens if the mechanical frequency equals the free-spectral range (FSR) in whispery-gallery modes (WGMs) [107]. In this case, there is a strong resemblance with the formation of OFCs in traveling-wave cavities as a result of third-order nonlinear interaction: the large density of states at resonant frequencies gives rise to a set of equidistant frequency lines at the output (Fig. 2.10(b)). 
(a)

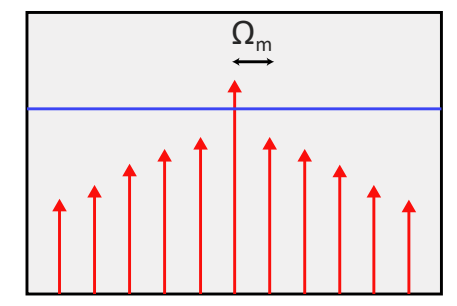

$\omega_{\mathrm{L}}$ (b)

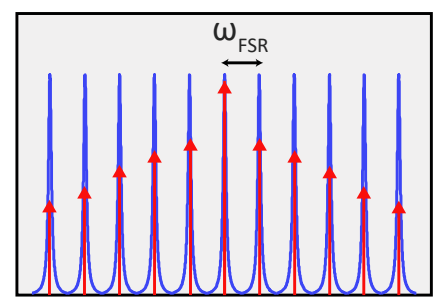

$\omega_{\mathrm{L}}$ (c)

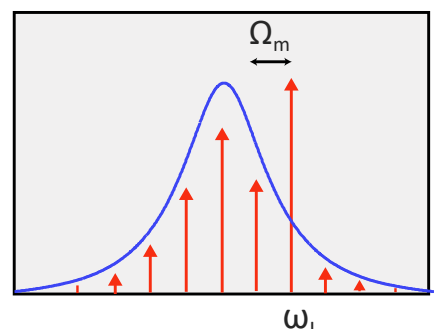

Figure 2.10: Generation of harmonics and OFCs in photonic structures. (a) In an optical waveguide, a cascaded Brillouin process gives rise to a set of harmonics spaced $\omega_{m}$ between them. (b) In a traveling-wave resonator supporting a series of high-Q WGMs with a Kerr nonlinearity, a set of harmonics is generated via four-wave mixing with a spacing $\omega_{F S R}$ from the laser frequency, where $\omega_{F S R}$ corresponds to the Free Spectral Range (FSR) of the resonator. (c) In an OM cavity, OM interaction induced by a bluedetuned laser scatters photons at frequencies $\omega_{m}$ apart from the laser frequency, which in a cascaded process generated more photons, giving rise to a series of harmonics, and ultimately to an OFCs occupying the cavity optical linewidth. In the figure, the blue curves stand for the photonic response of the structure and the red arrows show the different harmonics.

The generation of multiple harmonics in an OMC operated in the unresolved sideband regime inherits features of both effects (Fig. 2.10(c)). First, the cavity linewidth is not so broad as in the case of the waveguide, but the high density of states around the resonant frequency benefits the underlying OM interaction. In comparison with the WGM cavity, the OMC does not support multiple optical modes exactly spaced by the mechanical frequency, which greatly relaxes the conditions to get multiple harmonics. This way, in the output optical spectrum we expect a series of peaks at frequencies $\omega_{L}+m \omega_{m}$, with amplitudes $A_{m}$, being $m$ an integer number and $\omega_{L}$ the laser frequency. This closely resembles an OFCs of OM nature, which has been recently analyzed theoretically in [83]. Notice that, as in the case of OFC based on the Kerr-effect in traveling-wave optical resonators [122,123](Fig. 2.10(b)), here it is also a third-order nonlinear process that mediates in the OFC generation.

Since all generated photons are resonant within the same optical mode (Fig. 2.10(c)), the number of optical carriers forming the OFC is limited by the linewidth of the optical resonance. As suggested in Ref. [83], a cavity in the unresolved sideband regime should therefore provide wider OFCs than a cavity operating in the sideband-resolved regime. OM-generated OFCs have been previously observed using MHz-scale mechanical modes $[91,111,117,124]$. In this case, a high-Q cavity with a bandwidth $\approx 1 \mathrm{GHz}\left(\mathrm{Q}>10^{5}\right)$ can perfectly allocate a large number of harmonics. However, if we want to build an OFC from a GHz-scale fundamental harmonic, the $\mathrm{Q}$ factor of the cavity should be reduced to allow the build-up of a sufficient number of harmonics. According to that, in our case, the measured loaded optical Q factor satisfies the criteria in Ref. [83] to produce an OM-OFC with a spacing equal to $\Omega_{m} / 2 \pi$.

Hence, to perform the experiment, we blue-detuned our laser and recorded the detected RF spectra under different input powers. For an input power of $P_{i n}=3.38 \mathrm{~mW}$, we reach the threshold for the phonon lasing at $\lambda_{L}=1543.35 \mathrm{~nm}$, as in Fig. 2.11(a). 
(a)
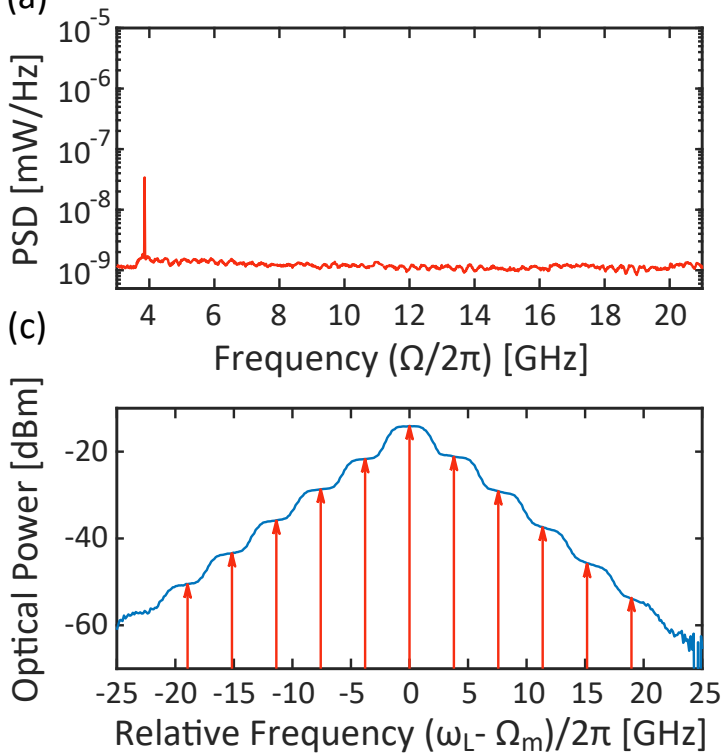

(b)
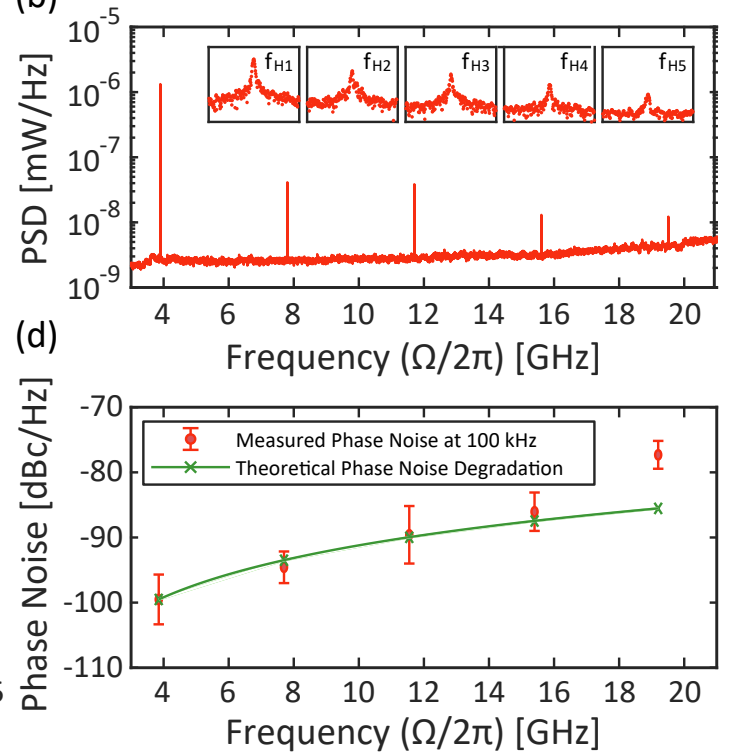

Figure 2.11: OFC generation in the OM crystal cavity at $P_{\text {in }}=3.38 \mathrm{~mW}$. (a) Detected RF spectrum above the phonon lasing threshold at $\lambda_{L}=1543.35 \mathrm{~nm}$; (b) RF spectrum at $\lambda_{L}=1544.37 \mathrm{~nm}$ where the $\mathrm{OFC}$ is produced. Five harmonics are observed, being higher-harmonic tones obscured by the thermal noise of the detection system. Close views of the five harmonics within a span of $200 \mathrm{MHz}$ are shown as insets; (c) Recorded optical spectrum (in blue) showing a set of peaks corresponding to different harmonics represented (in red) at the expected position. (d) Phase noise of the different harmonics of the OFC. In red, the mean value of the phase noise for different phase noise measurements is presented with its standard error and in green. The theoretical phase noise degradation of $20 \cdot \log (m)$ with respect to that of the 1st harmonic is also shown.

When the laser wavelength is increased until $\lambda_{L}=1544.37 \mathrm{~nm}$, higher-order harmonics are generated, thus resulting in an OFC, whose first five harmonics are shown in Fig. 2.11(b). Notice that going from the phonon lasing to the OFC regime requires not only an increase of the input power, but also a larger wavelength of the driving laser because of the red-shift of the optical resonance because of the thermo-optic effect. Fig. 2.11(c) shows the optical spectrum of the generated OFC. The width of the peaks observed in the optical spectrum is limited by the resolution of the optical spectrum analyzer, which is $0.01 \mathrm{~nm}(1.2 \mathrm{GHz})$. When photodetected, the optical peaks are beaten resulting in the multiple peaks as seen in Fig. 2.11(b). Notice that every peak results from the addition of several beats. For instance, the peak at $2 \Omega_{m}$ results from summing up the beating notes $\omega_{L}+2 \Omega_{m}$ with $\omega_{L}, \omega_{L}-2 \Omega_{m}$ with $\omega_{L}, \omega_{L}+\Omega_{m}$ with $\omega_{L}-\Omega_{m}$, and all the other optical peaks that are spaced exactly that frequency. However, for any frequency, the terms including a beating of $\omega_{L}$ will dominate.

We also measured the phase noise of the different harmonics, as shown in Fig. 2.11(d). In principle, the harmonic mixing process will result in an added phase noise of $20 \times$ $\log (m)$ with respect to that of the first harmonic. It can be seen that the previous rule is well satisfied in our device. Notice that this is in stark contrast with the Brillouin OMO in high-Q cavities, for which higher harmonics show a better noise performance because the Brillouin process purifies the laser beam [107]. This is because the device in 
Ref. [107] operates in the regime where the photon lifetime exceeds the phonon lifetime in the cavity, which results in a narrowing of the Stokes line [82]. In our case, we operate in the opposite scenario, being optical losses larger than mechanical losses so the scattered Stokes wave is essentially a frequency-shifted copy of the laser beam plus an extra phase noise added by the mechanical oscillator [82]. Thus, the cavity acts as a harmonic generator (comb) and the phase noise behaves as in a conventional harmonic mixer, being the phase noise impaired by the factor previously mentioned. Even though the harmonic mixing process results in an added phase noise of $20 \times \log (m)$ experimental measurements show noise figures $<-80 \mathrm{dBc} / \mathrm{Hz}$ above $15 \mathrm{GHz}$.

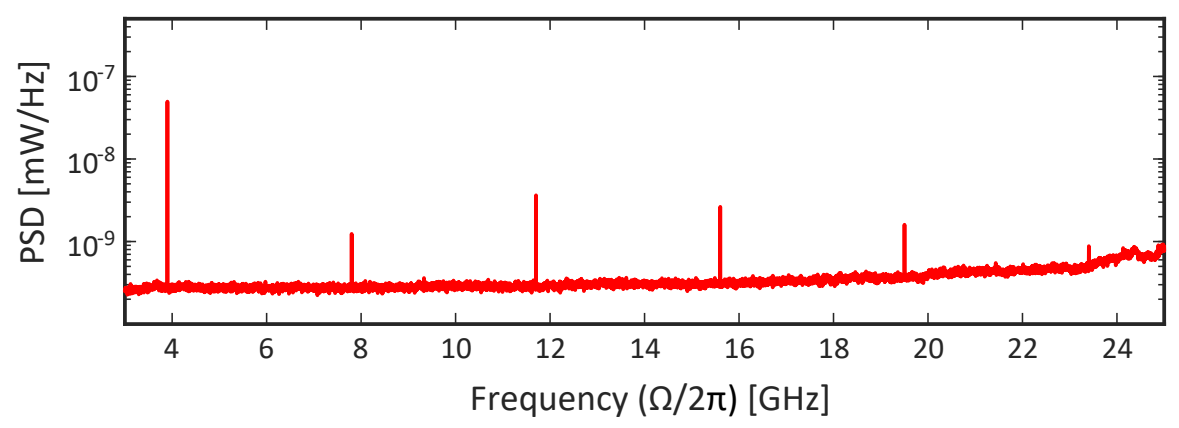

Figure 2.12: PSD spectrum of the OFC generated with the OM crystal cavity up to the 6th harmonic.

In the previous figures, we show up to the fifth harmonic, but higher harmonics can be reached as can be seen in Fig. 2.12. Improving the signal to noise ratio (SNR) may help in the observation of even higher order harmonics. It may be also pointed out that these measurements were performed with a $50 \mathrm{GHz}$-bandwidth Photodetector $\left(u^{2} t\right.$ XPDV2120R) with low sensitivity, so increasing the sensitivity of the system should also contribute to increase the SNR ratio. For more details regarding the experimental setup see Section 2.5.1.

\subsubsection{Temporal OFC dynamics}

As an in-depth study of the OFC dynamics, we studied the optical traces of the generated OFC in the time domain. The dynamics of OM frequency combs can be described by the next set of coupled equations that model the time evolution of the optical mode amplitude $a$ and the amplitude of motion $x$ as [2,83]:

$$
\begin{gathered}
\frac{d a}{d t}-\left(i(\Delta+G x)-\frac{\kappa}{2}\right) a=\sqrt{\kappa_{e}} s_{i n} \\
\frac{d^{2} x}{d t^{2}}+\Gamma_{m} \frac{d x}{d t}+\Omega_{m}^{2} x=\frac{\hbar G}{m_{e f f}}|a|^{2}
\end{gathered}
$$

where $\Delta$ is the detuning of the laser from the optical resonance, $G$ is the optical frequency shift per displacement unit, $\kappa$ is the overall intensity decay rate, $\kappa_{e}$ represents the input coupling losses, $s_{i n}$ is the input photon flux and $m_{e f f}$ is the effective mass of the mechanical oscillator. In the blue-detuned regime $(\Delta>0)$ the OM damping rate $\Gamma_{o p t}$ becomes negative, decreasing the overall damping rate $\Gamma_{\text {eff }}$ that first leads to heating of 
the oscillator, as we studied before. When the overall damping rate finally becomes negative, an instability appears producing an exponential grown of any fluctuation, which will finally saturate by nonlinear effects giving rise to a phonon lasing regime [2]. The saturation of these self-induced oscillations can be seen in Fig. 2.13(a) which was obtained from the numerical simulations of the OM coupled equations Eq. 2.16 and Eq. 2.17. A close view of the saturated regime is shown in Fig. 2.13(b).

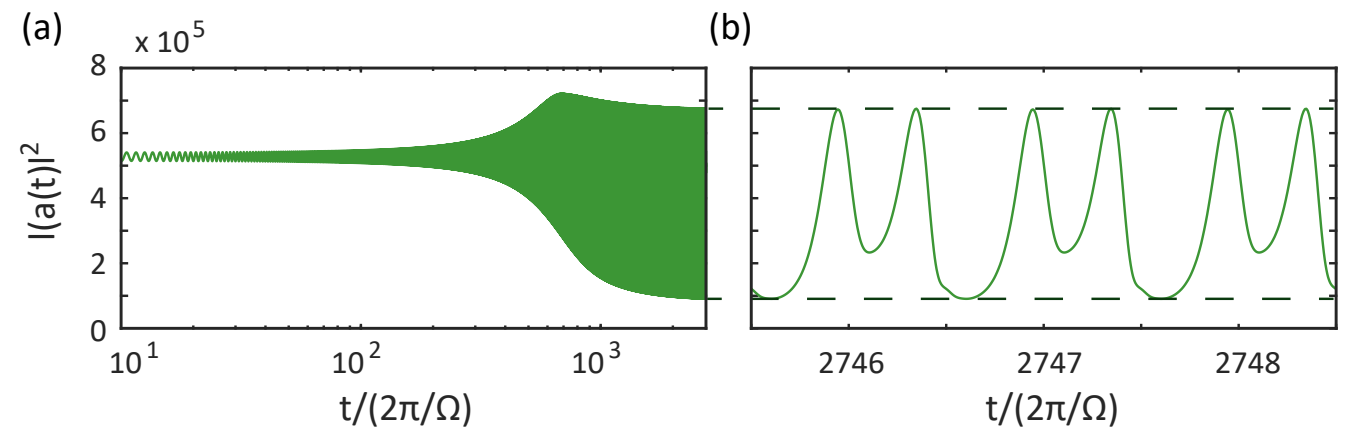

(b)

Figure 2.13: (a) Simulated self-induced oscillations showing the evolution of the intracavity photons versus time in logarithmic scale. (b) Close view of the saturated oscillation with a stable amplitude corresponding to the optical traces of the OFC.

The experimental traces were acquired by photodetecting the transmitted optical signal via a high-speed and high-sensitivity photoreceiver whilst the reflected signal was used to trigger the waveform in a high-speed oscilloscope. Both, the theoretical and experimental temporal traces are presented in Fig. 2.14 for different laser wavelengths corresponding to different detunings - and input photon fluxes $\left|s_{i n}\right|^{2}$ — placed between $0.9 \times 10^{17}$ and $1.7 \times 10^{17} \mathrm{~s}^{-1}$. These input photon fluxes, defined as the rate of photons arriving at the cavity, are related to the input powers presented before as $P_{i n}=\hbar \omega_{L}\left|s_{i n}\right|^{2}$ [2]. It has to be noted that, despite the optical resonance was originally placed at a wavelength equal to $1522.5 \mathrm{~nm}$, we were able to shift it up to values as higher as 1550 $\mathrm{nm}$, as in the recorded traces reported here, due to the thermo-optic effect [67]. This effect causes a typical bistability "saw-tooth" shaped transmission, which gets both broader and shallower when the input power is increased [125]. In this regime, it is impossible to know accurately the position of the optical resonance, and, as a result, we cannot know the optical detuning $\Delta$. Because of that, the detuning, shown in the temporal traces of Fig. 2.14, has been set as a fit parameter in order to compare the theoretical and experimental optical traces.

The remaining cavity parameters required in the theoretical model were obtained from the simulation of the fabricated structure. In particular, we used a mechanical zero-point fluctuation amplitude $X_{z p f}=2.69 \cdot 10^{-15} \mathrm{~m}$ and $m_{\text {eff }}=2.7 \cdot 10^{-16} \mathrm{~kg}$, obtained from the OM crystal cavity simulations. For the overall decay rate, we introduced the experimental measurements of $\kappa / 2 \pi=39 \mathrm{GHz}$ and $\kappa_{e}=0.26 \kappa$. Figure 2.14 shows a nice agreement between the experiments and the theoretical model. Different waveforms can be obtained by modifying either the detuning or the input power, as pointed out in Ref. [83]. We also observed different waveforms for other driving conditions, being highly reproducible using the theoretical model. This confirms that OMCs can be used to synthesize microwave waveforms beyond the generation of single microwave tones and, therefore, they can play a role in the development of microwave photonics applications 
(a)

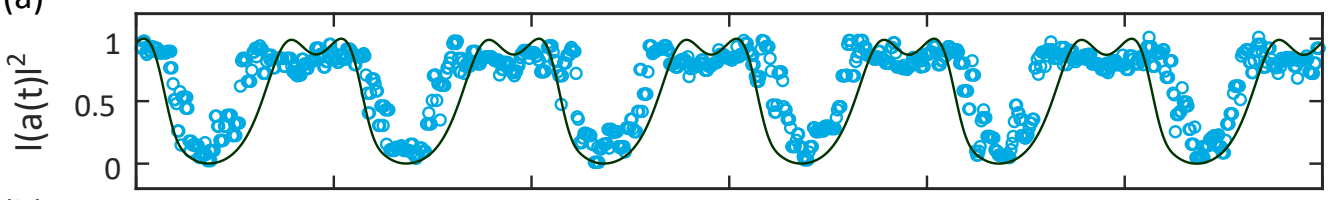

(b)

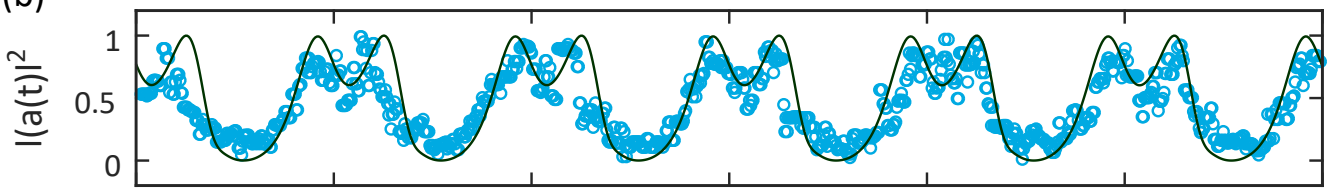

(c)

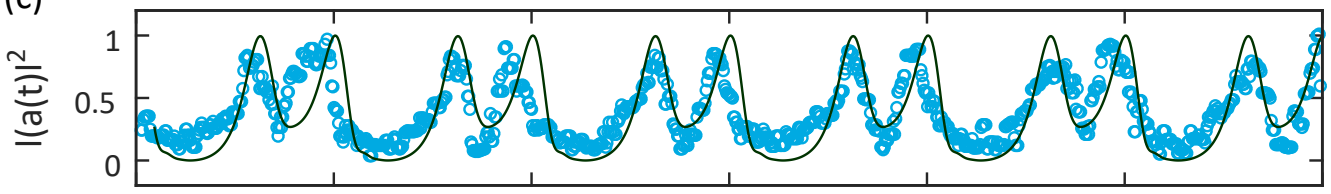

(d)

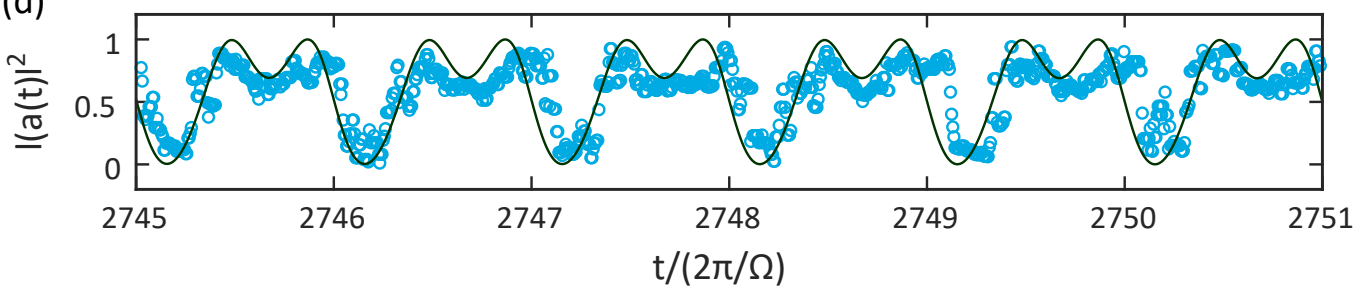

Figure 2.14: Comparison of the experimental (blue dots) and theoretical (solid line) temporal OM-generated OFC traces under different experimental conditions. (a) $\lambda_{L}=1543.35 \mathrm{~nm}$ (experiment) and $\Delta=4 \Omega_{m}$ (simulation); (b) $\lambda_{L}=1544.05 \mathrm{~nm}$ (experiment) and $\Delta=3.4 \Omega_{m}$ (simulation); (c) $\lambda_{L}=1545.37 \mathrm{~nm}$ (experiment) and $\Delta=2.4 \Omega_{m}$ (simulation); (d) $\lambda_{L}=1545.62 \mathrm{~nm}$ (experiment) and $\Delta=\Omega_{m}$ (simulation).

in silicon photonics technology. It mus be pointed out that the main results presented in this chapter can also be found at Ref. [101].

\subsection{Experimental setups}

In this section, we describe the experimental setups used in the acquisition of the measurements presented in this chapter. However, it must be pointed out that the optical and mechanical characterization scheme and the OM coupling rate measurement setup will be equal for the next chapters.

\subsubsection{Optical and mechanical cavity characterization}

The overall optical and mechanical cavity response has been measured with the experimental setup depicted in Fig. 2.15(a). By overall optical and mechanical cavity response we mean the measurements of the optical and mechanical driven resonances, the different mechanical states that the cavity experience and the temporal trace measurements.

First, a continuous wave is generated with a tunable fiber-coupled external cavity diode laser (New Focus Velocity TLB-6728). Then, the optical signal is amplified by means of an erbium doped fiber amplifier (EDFA) to fulfill our power requirements and a polarization controller $(\mathrm{PC})$ sets the required polarization that will finally arrive at 
the device under characterization. After the polarization controller, the optical wave is sent through a circulator to a closed part of the system where a tapered fiber loop and the OM cavity are placed. In figure 2.15(a) only a not-scaled schematic of the loop and the cavity is presented, but this closed region will be broadly described in Fig. 2.15(b).

Once the optical signal arrives at the tapered micro-loop fiber, it can couple light in and out of the cavity. However, this only happens if the loop is close enough to it, as it couples the light into the cavity via evanescence waves. The relative position between these two elements can be tuned with piezoelectric controllers placed at the sample holder where the cavity is located. This coupling scheme enables us to characterize both the transmission and the reflection response.

Regarding the transmission channel, we switch between two different configurations as a function of the measurement to be performed. For optical spectrum measurements, as in Fig. 2.11(c), the top red configuration is selected. Here, the signal is first amplified, if needed, with an EDFA and then it is analyzed through an optical spectrum analyzer (OSA)(ANDO AQ6317C). On the other hand, time varying optical traces and the optical resonance measurements are measured with the bottom configuration. In the case of the optical resonance measurements, the signal is attenuated with a VOA, and then it is photodetected via a switchable gain amplified detector (Thorlabs PDA20CS-EC InGaAs Switchable Gain Amplified Detector) and finally recorded with an oscilloscope (Scope)(DSO81204B Infiniium High Performance Oscilloscope). Nevertheless, for the temporal traces also a trigger signal is needed in order to record correctly the time varying traces. This trigger signal is obtained through the reflection channel and it is measured with a Digital Communications Analyzer DCA-J 86100C in oscilloscope mode. The used PD is in this case a $12 \mathrm{GHz}$ photodetector (PD).

The reflection channel consists of either a $12 \mathrm{GHz}$ Photodetector (New Focus 1544-B DC-Coupled NIR Fiber-Optic Receiver) or a $50 \mathrm{GHz}$ Photodetector $\left(u^{2} t\right.$ XPDV2120R) in order to record higher-order harmonics. It has to be noted that the $12 \mathrm{GHz}$ bandwidth detector has more sensitivity, yet it can only measure up to the third harmonic. Once the signal has been photodetected we can choose either to analyze it through an RF spectrum analyzer (RSA) or use it as the trigger signal for the temporal trace measurements. It must be remarked that different RSA has been along with the experiments: one for the analysis of the mechanical response of the cavity (Rohde \& Schwarz FSQ 40) and another for the phase noise measurements (Rohde \& Schwarz FSUP signal source analyzer).

Concerning the previously mentioned closed part of the system, a schematic representation can be seen in Fig. 2.15(b). It consists of a methacrylate cage structure where both the tapered micro-looped fiber and the sample are located. This closed environment is where the taper fiber and the loop are also fabricated (for more details regarding this fabrication process see Appendix B). The relative position between the sample and the loop can be tuned through the stage where the sample holder is placed, where a fine tune can be performed via piezoelectric controllers. Also, a lateral and a vertical microscope (LM and VM, respectively) are used in the coupling process. Image views from these two microscopes can be seen in the insets panels in Fig. 2.15(b).

\subsubsection{Optomechanical coupling rate measurement}

In regards to the measurements of the OM coupling rate $\left(g_{0}\right)$, we use the calibration tone technique [65,68], whose experimental setup is shown in Fig. 2.16. This method consists of the comparison between the power spectral density resulting from the mechanical 
(a)

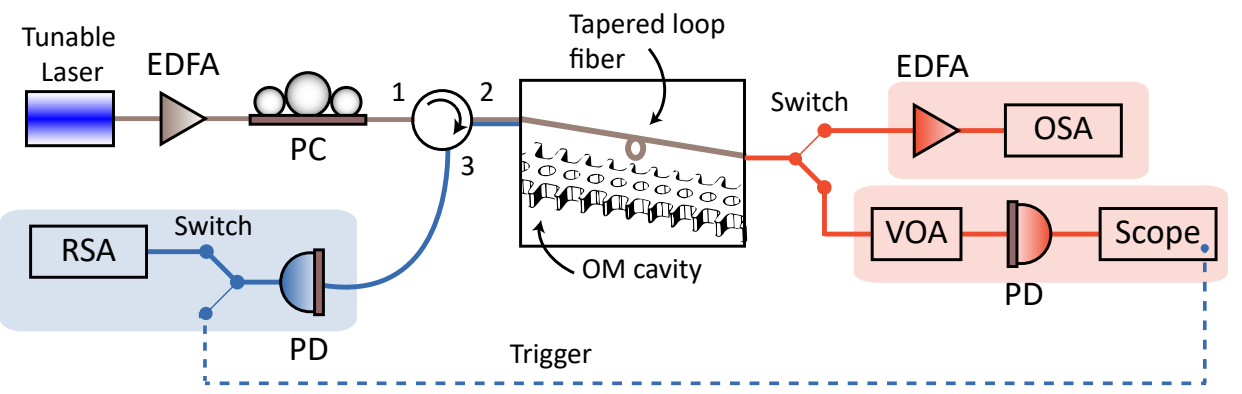

(b)
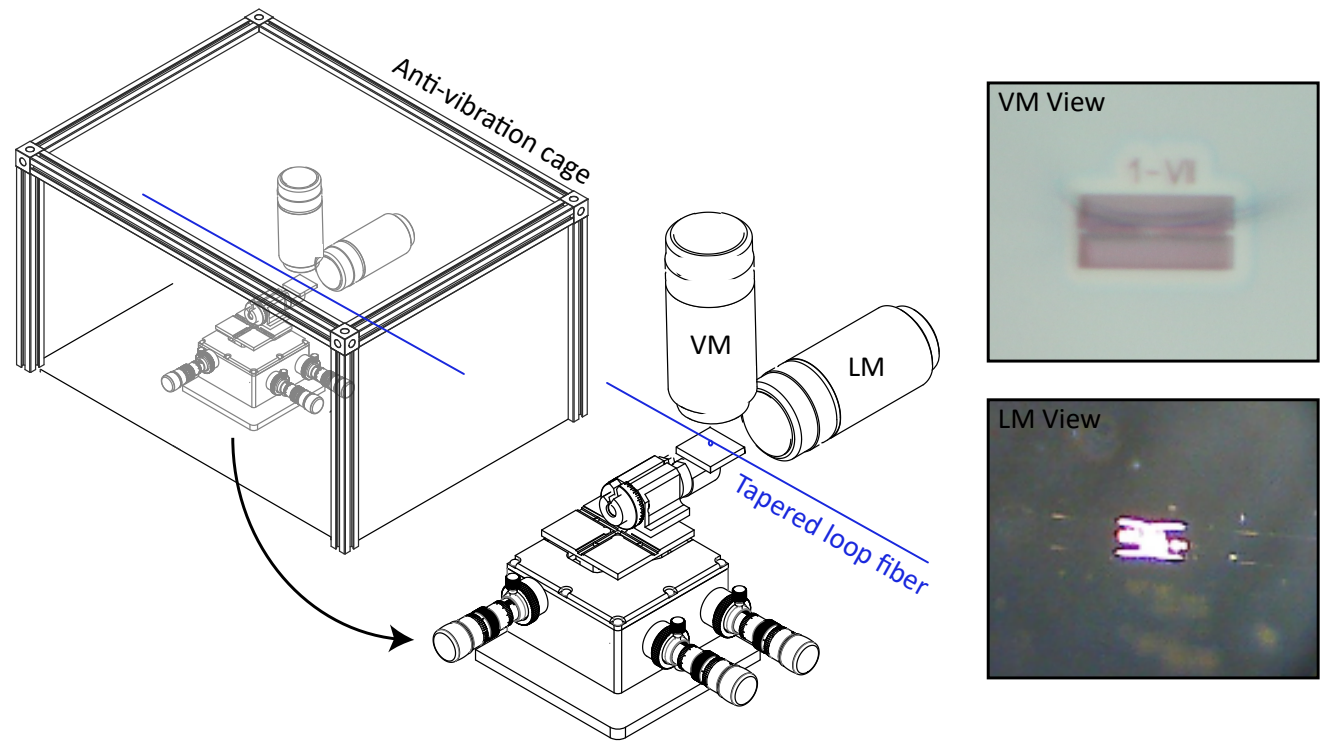

Figure 2.15: Sketches of the experimental setups used in the OM characterization of the cavities, which were coupled via evanescent waves to a tapered fiber loop. The box containing the cavity and fiber loop is not to scale but zoomed for illustration purposes. (a) A tunable laser generates a continuous wave (CW) optical signal that passes through a variable optical attenuator (VOA), a polarization controller and an optical circulator before entering the OM cavity. In reflection (port 3 of the circulator) we include a 12 $\mathrm{GHz}$ bandwidth photodetector (PD) and a radiofrequency spectrum analyzer (RSA). In transmission, the signal is first split up using a 90:10 splitter: part of the signal is amplified by an EDFA and then injected into an optical spectrum analyzer (OSA); the second path is photodetected to measure the level of the signal. (b) Schematic representation of the closed part of the system. It contains the sample and sample holder and two microscopes for coupling purposes, a vertical and a horizontal one. Photo views of each microscope are presented as insets. In the top view from the vertical microscope (VM) the lower part of a tapered loop coupled to an OM cavity under characterization is shown. In the lateral view from the lateral microscope (VM) an illuminated set of samples and a fiber loop is presented. 
oscillator's thermal noise and the one obtained from a calibration tone. The calibration tone is created by phase-modulating the input laser light from a tunable laser, whose polarization is first optimized to maximize the transmitted signal. The phase modulation is performed with a fiber-coupled electro-optic phase modulator $(\mathrm{PM})(\mathrm{EOspace}$ Phase Modulator) with a known modulation depth with an applied driving voltage of $V_{p}=0.07$ $\mathrm{V}$ at $\Omega_{c} / 2 \pi=3.91 \mathrm{GHz}$ and a half-wave voltage $V_{\pi}=3.1 \mathrm{~V}$. The modulation depth accounts for the extinction ratio of the phase offset of the light as $\Phi / \pi=V_{p} / 2 V_{\pi}$. The calibration generation part is shadowed in yellow in Fig. 2.16.

Afterward, the phase-modulated light is sent to the cavity under characterization as described in Section 2.5.1. For this particular configuration, the transmission channel is used to characterize the mechanical response of the cavity and the reflected signal is used to monitor and characterize the optical response.

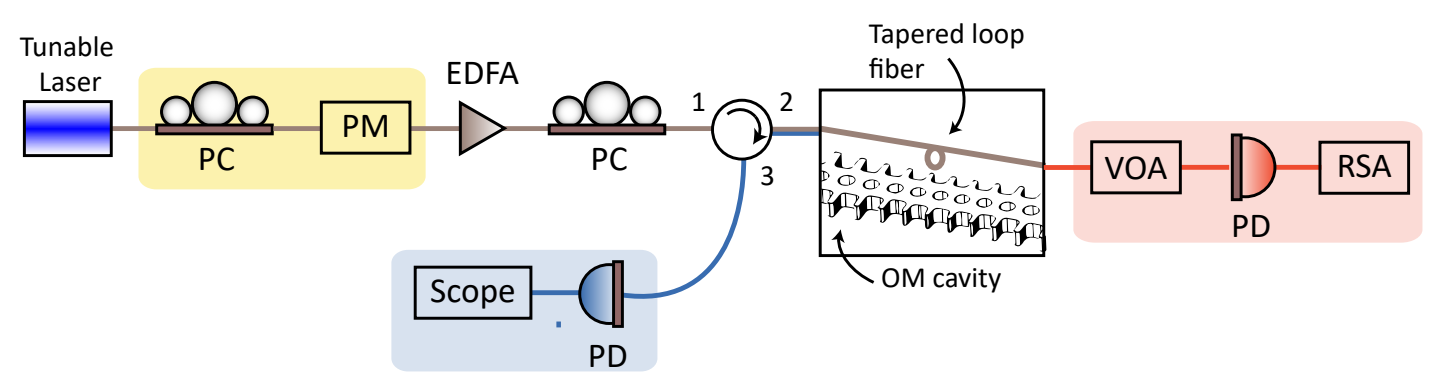

Figure 2.16: A laser is modulated with a microwave tone via a phase modulator in order to introduce a calibration tone to estimate the OM coupling rate.

Finally, comparing the PSD area of both the mechanical mode response $\left(\left\langle V^{2}\right\rangle_{m}\right)$ and the calibration tone $\left(\left\langle V^{2}\right\rangle_{c}\right)$ we calculate $g_{0}$ as [68]:

$$
g_{0}=\left(\frac{V_{p}}{V_{\pi}} \pi\right) \frac{\Omega_{c}}{2} \sqrt{\frac{1}{n_{t h}} \frac{\left\langle V^{2}\right\rangle_{m}}{\left\langle V^{2}\right\rangle_{c}}}\left|\frac{G_{v \omega}\left(\Omega_{c}\right)}{G_{v \omega}\left(\Omega_{m}\right)}\right|
$$

where $n_{t h}$ is the number of phonons occupying the mechanical mode at room temperature and $G_{v \omega}\left(\Omega_{i}\right)$ is the frequency dependent transduction factor. As the calibration tone was placed just a few $\mathrm{MHz}$ apart from the mechanical resonant mode it can be assumed that both transduction factors are nearly the same so $\left|G_{v \omega}\left(\Omega_{c}\right) / G_{v \omega}\left(\Omega_{m}\right)\right| \simeq 1$. An overview of the measurement can be appreciated in Fig. 2.17(a) with both the mechanical mode and the calibration tone just a few $\mathrm{MHz}$ apart from each other. It must be noted that the area of the signal $\Omega_{m}$ has been calculated through a Lorentzian shape fit and the calibration tone $\Omega_{c}$ through a Gaussian one.

In the different $\mathrm{OM}$ coupling rate measurements performed along this Thesis we have used two different phase modulators: EOSpace Phase Modulator (measurements presented in Chapter 2) and EOSpace Phase Modulator PM-0K5-10-PFA-PFA (measurements presented in Chapter 4). Despite the half-wave voltage $V_{\pi}$ is a commonly given parameter, it can change as a function of the frequency so a further characterization of the used phase modulators was performed in order to ensure the used parameters. With the measurement of $V_{\pi}$ as a goal, the light transmitted through the phase modulator was then sent to an optical fiber cavity able to resolve both the carrier $\left(A_{c}\right)$ and sideband $\left(A_{s}\right)$ amplitudes of the signal. Once both the carrier and sideband were time-resolved, the obtained amplitudes were recorded as a function of $V_{p}$ for the RF 

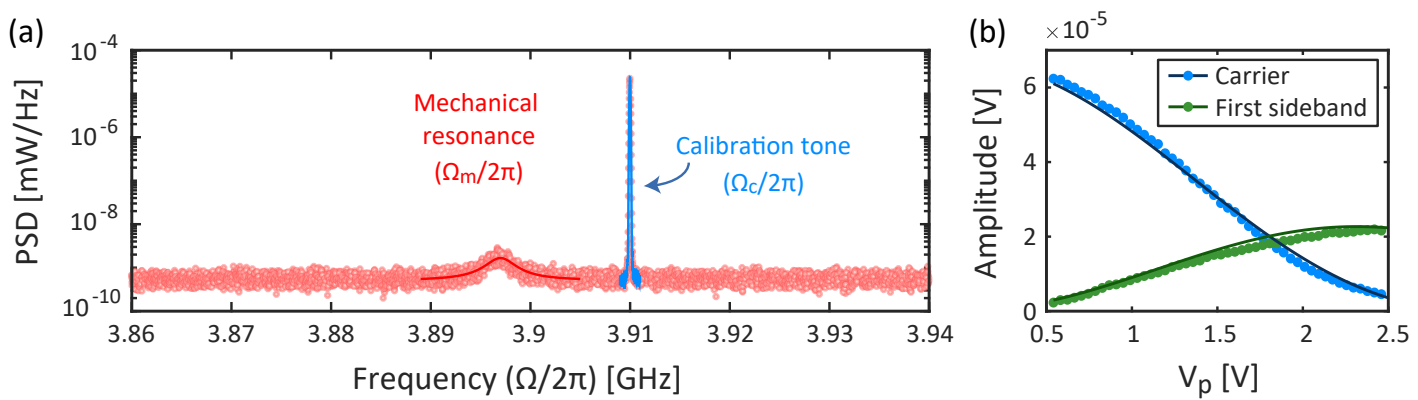

Figure 2.17: (a) Calibration tone method overview for the determination of the OM coupling rate showing a Lorentzian curve fit of the mechanical mode(red) and the Gaussian shape fit of the calibration tone (blue). (b) Carrier and first sideband variation of the phase modulated light as a function of the applied driving voltage at $\Omega_{c} / 2 \pi$.

frequency drive under study. Therefore, as both the carrier and sideband amplitude signals can be described by the Bessel functions $J_{0}$ and $J_{1}$ as $A_{c}=a\left(J_{0}\left(\pi V_{p} / V_{\pi}\right)^{2}\right.$ and $A_{s}=a\left(J_{1}\left(\pi V_{p} / V_{\pi}\right)\right)^{2}$, respectively, a fit of the evolution of the amplitudes to the Bessel functions was performed, as can be seen in Fig. 2.17(b), thus retrieving the $V_{\pi}$ value at the required frequency. Here, $a$ is a scaling factor. 


\section{Chapter 3}

\section{All-optical frequency conversion}

\section{Contents}

3.1 Introduction $\ldots \ldots \ldots \ldots \ldots \ldots \ldots \ldots \ldots \ldots \ldots$

3.2 Long-term stability $\ldots \ldots \ldots \ldots \ldots \ldots \ldots \ldots$

3.3 Frequency conversion in the lasing regime $\ldots \ldots \ldots 38$

3.3.1 Data stream modulation using OFDM . . . . . . . . . . . 39

3.3.2 Downconversion using the first harmonic of the mechanical resonance . . . . . . . . . . . . . . . 4 40

3.3.3 Upconversion for higher order harmonics . . . . . . . . . . . . 43

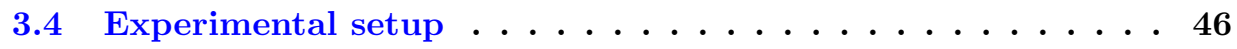

3.4 .1 OFDM Frequency conversion setup . . . . . . . . . 46

\subsection{Introduction}

As it was described in previous chapters, when operating in the phonon lasing regime, the mechanical vibration can efficiently modulate the input optical carrier by carefully detuning the driving laser towards the blue side of the optical resonance. This feature could be of use in microwave photonics, which involves the manipulation and processing of RF and microwave signals, entirely in the optical domain by using photonic devices [39]. As already mentioned, this has multiple advantages compared to the processing of such signals in the electric domain, including nearly-unlimited bandwidth, immunity to electromagnetic interference or frequency-independent losses. Therefore, the OMC acting as an OMO could be a key building block in microwave photonics, addressing the efficient processing of microwave signals in the optical domain [39]. In particular, the use of OMCs could be disruptive in the way towards the desired on-chip integration of such microwave photonic components [126] because no other approach permits all-optical microwave processing in wavelength-scale foot-prints [40].

$\mathrm{OM}$ interaction is a nonlinear process itself. This means that, beyond their use as microwave oscillators already discussed in Section 2.3, OMCs can also be employed to perform frequency conversion of RF signals in the optical domain. Frequency conversion or mixing is a key process in any communication system, particularly in wireless communications where the information must be transmitted modulated in a certain frequency band (typically, in the microwave domain). For instance, in WiFi networks, data streams 
generated in computers are transmitted at either 2.4 or $5.7 \mathrm{GHz}$ frequencies depending on the employed standard. Typically, electrical frequency conversion involves the use of a mixer, which is a three-port nonlinear device, and a local oscillator, plus certain amplifiers and filters, as can be appreciated in Fig. 3.1.

In the transmitter end, it is mandatory to perform frequency up-conversion of the data streams, which are modulated on a low-frequency carrier called intermediate frequency (IF) (Fig. 3.1(a)). This signal is mixed with the local oscillator (LO) to produce an upconverted signal (US) at the output at a frequency $\Omega_{U S}=\Omega_{R F}=\Omega_{L O}+\Omega_{I F}$. This signal passes through an RF filter that removes undesired frequency components and then it is amplified before being radiated to the free space by a certain antenna. The downconversion process, Fig. 3.1(b), takes place in reception: the RF signal is received by an antenna and amplified before reaching the mixer. Again, a LO is used in the mixing process to get a downconverted signal (DS) with frequency $\Omega_{D S}=\Omega_{I F}=\Omega_{R F}-\Omega_{L O}$. Notice that it is usual to extract the LO from the received RF signal in order to ensure coherence. In Fig. 3.1, we can see both the standard electrical conversion scheme as well as some panels showing the expected frequency signals at each port of the mixer. As a reminder, we define $\Omega_{i}=2 \pi f_{i}$.

(a)
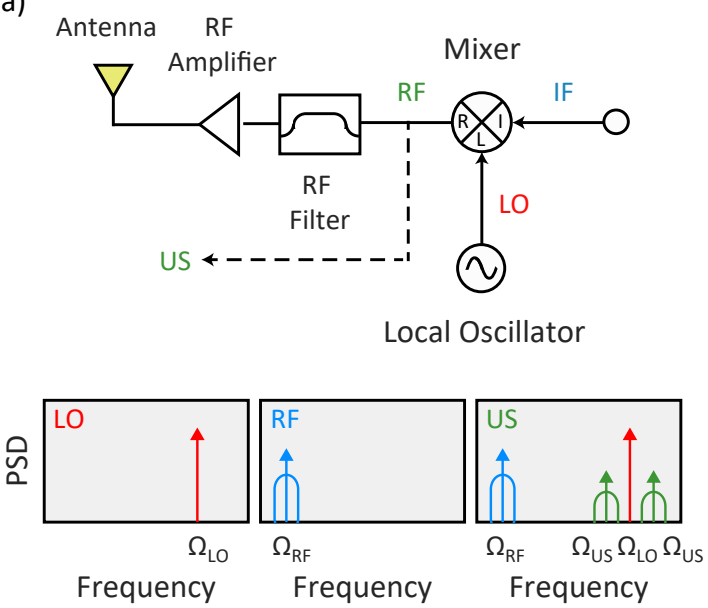

(b)
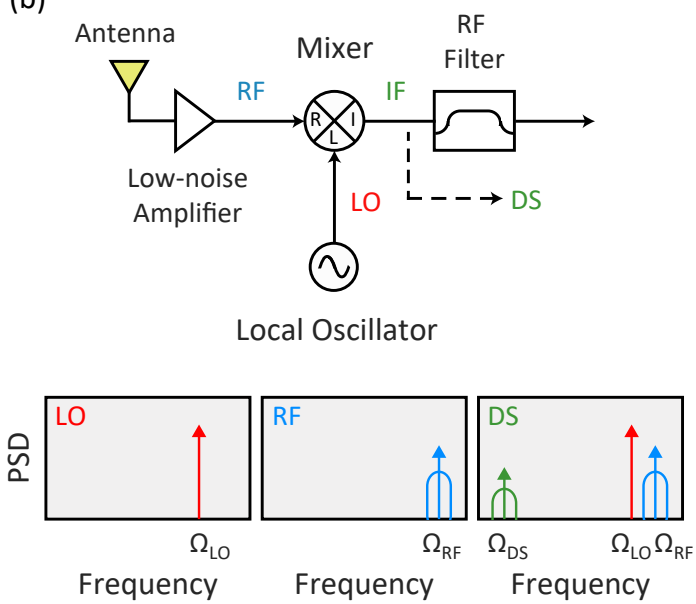

Figure 3.1: (a) (Top) Schematic diagram of a standard electrical upconverter using a mixer and a local oscillator and (Bottom) their frequency signal representation at each mixer port. (b) (Top) Schematic diagram of a standard electrical downconverter using a mixer and a local oscillator and (Bottom) their frequency signal representation at each mixer port.

Photonics-based frequency mixing has many benefits in comparison with the standard electrical approach. Many different architectures have been reported in the literature, as described in the recent review [40]. Elements such as semiconductor optical amplifiers or Mach-Zehnder modulators are used to perform the mixing, being always an LO required. Remarkably, in our case, the OMO utilizes its own LO in the mixing process [42], thus playing the role of both the mixer and the LO in the schemes presented in Fig. 3.1. This results in a feature that could have enormous benefits in comparison with standard mixing approaches used in microwave photonics, since it avoids the use of LOs, which can be very expernsive for high frequencies [40]. This characteristic was employed more 
than one decade ago to demonstrate down-conversion of $25 \mathrm{MHz}$ RF tones modulating an optical carrier using a silica microtoroid as OMC [41]. Also, frequency mixing at $\mathrm{MHz}$ frequencies was demonstrated in a torsional OM resonator via dissipative coupling, which does not requires self-sustained oscillations [43]. Conversely, to the best of our knowledge, the operation with realistic data streams modulated at $\mathrm{GHz}$ frequencies as in current wireless networks and systems has not yet been demonstrated.

Therefore, in this chapter, we demonstrate all-optical frequency conversion of data streams employing orthogonal frequency division multiplexing (OFDM) modulation by driving the OMC presented in Chapter 2 into the phonon lasing regime. Particularly, we demonstrate the successful conversion of full-standard IEEE 802.16 WiMAX signal with QPSK modulation over different bandwidths with a conversion efficiency better than $-17 \mathrm{~dB}$ in both processes and without requiring an external LO. WiMAX (Worldwide Interoperability for Microwave Access) is a widely employed system for wireless communications over broad areas, being available in both fixed and mobile versions [127]. Indeed, the mobile version, based on the standard IEEE $802.16 \mathrm{e}$ was originally a competitor of Long Term Evolution (LTE) in the race towards the massive deployment of $4 \mathrm{G}$ mobile networks. Regarding the upconversion system, we study how the upconverted signal evolves as a function of the used OFC harmonic. Finally, we also explore the possibility of convert 16QAM modulation signals in both conversion systems. This paves the way towards the use of silicon photonics chips in compact high-capacity next-generation wireless networks.

\subsection{Long-term stability}

Besides the OMO short-term stability analysis given in Section 2.3 through the phase noise analysis, a long-term stability characterization may also be needed for practical applications. In other words: in a real application, such in wireless networks, we need our cavity to operate with the same performance for very long periods of time. The longterm stability can be quantified by the Allan deviation $\sigma_{y}(\tau)$ term for long averaging times, which can provide information about the frequency drift of our oscillator. In order to calculate this, we follow a different approach to the one used in Chapter 2 where the Allan deviation was calculated through the phase noise spectra, which is an approximation [112] as discussed in Section 2.3.3. Nevertheless, this is not the case if we analyze it from the original meaning. To that end, $\sigma_{y}(\tau)$ can be defined as the variance of the difference between two fractional frequency values $y_{i}$ and $y_{i+1}$ measured at $t_{0}+i \tau$ and $t_{0}+(i+1) \tau$. The Allan variance $\sigma_{y}^{2}(\tau)$ depends on the variable $\tau$ and expresses the mean square of all frequency counter samples separated in time by $\tau$ over the entire measurement interval $T$ [128], as depicted in Fig. 3.2(a). Here, it is presented the evolution of the first harmonic frequency in the lasing regime as a function of time over the total measurement time of $120 \mathrm{~s}$. The Allan deviation can be thus calculated as:

$$
\sigma_{y}(\tau)=\sqrt{\frac{1}{2 M} \sum_{i=0}^{M-1}\left(y_{i+1}-y_{i}\right)^{2}}
$$

where $M=T / \tau-1$ and $y_{i}=\left(\left\langle f_{m}\left(t_{0}+i \tau\right)\right\rangle_{\tau}-\nu_{0}\right) / \nu_{0}$. Here, $\left\langle f_{m}\right\rangle_{\tau}$ is the average frequency of the system over the interval $\tau$ and $\nu_{0}$ the frequency of the reference oscillator. 

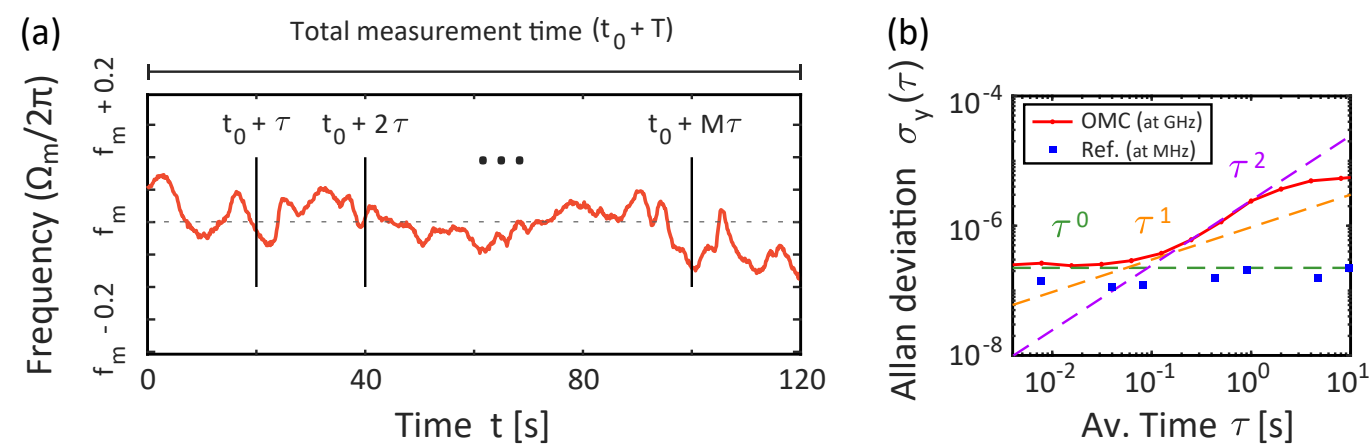

Figure 3.2: (a) Evolution of the first harmonic frequency versus time within a span of $400 \mathrm{kHz}$. (b) Long-term Allan deviation calculated from the time evolution of the mechanical mode (blue) and non-stabilized nanomechanical beam at $\mathrm{MHz}$ from [129].

This calculation has some limitations regarding the measurement uncertainty, which depends not only on the frequency accuracy of the spectrum analyzer but also on the accuracy of $\tau$. Because of that, this method is not recommended for small $\tau$ [128], thus resorting to the phase noise derivation method. In our long-term stability experiments, the oscillator frequencies were measured by a real-time spectrum analyzer with a sampling rate $\delta t$, thus resulting in an Allan deviation described by [130]:

$$
\sigma_{y}(\tau=m \delta t)=\sqrt{\frac{1}{2(N-m)} \sum_{i=0}^{N-m-1}\left(\frac{f_{m}(k+m) \delta t-f_{m}(k \delta t)}{\nu_{0}}\right)^{2}}
$$

with $m=1,2, \ldots, N$.

The final retrieved long-term $\sigma_{y}(\tau)$ is shown in red in Fig. 3.2(b). Here, different noise sources as the flicker frequency noise $\left(\sigma_{y}(\tau) \propto \tau^{0}\right)$ and a very small contribution of the random walk of frequency $\left(\sigma_{y}(\tau) \propto \tau^{1}\right)$ can be appreciated. It must be noted that the cavity where this measurement was performed was nominally the same design as in Chapter 2 but it was a different fabricated cavity. In particular, in the cavity where the long-term measurements were performed there was also another mechanical mode confined in the cavity, thus explaining a slightly worse obtained $\sigma_{y}(\tau)$ for the flicker frequency noise contribution. This cavity and the obtained results will be described broadly in the next chapter, where accordance between both derivation methods will be presented. Besides that, also a linear frequency drift contribution with $\sigma_{y}(\tau) \propto \tau^{2}$ is present. Comparing the obtained values with others found in the literature, we found that they are on par with other nanomechanical oscillators [129], as presented in blue in Fig. 3.2(b). Indeed, even though the oscillator in [129] performs a little bit better, it oscillates at much lower frequencies, so we can think that in a qualitative way they perform similarly.

\subsection{Frequency conversion in the lasing regime}

As it was stated before, we drive the OMC already presented in Chapter 2 into the lasing regime, hence it can act as a OMO. In this regime, the oscillator can be employed as an 
RF mixer where the LO is inherently included in the cavity.

\subsubsection{Data stream modulation using OFDM}

Concerning the operation of real data streams in wireless communication systems, the frequency conversion of digitally modulated signals employing OFDM modulation is of special interest. OFDM is a digital modulation method that encodes data on multiple orthogonal carriers, called sub-carriers. Each sub-carrier is modulated with quadrature amplitude modulation (QAM) with a certain number of symbols $(M) . M$ is the number of possible combinations of amplitude and phase of a certain sub-carrier. In its simplest form, $M=4$, being this modulation also called Quadrature Phase-Shift Keying (QPSK). In real systems, $M$ is dynamically changed in an adaptive way: when the channel is good, $M$ can become very large and the transmission rate (in bit/s) is increased; when there are some impairments that reduce the signal-to-noise ratio, $M$ is reduced at the expenses of a poorer transmission rate. The main advantages of OFDM over singlecarrier schemes are the spectral efficiency achieved, and its ability to cope with severe channel conditions, being resistant to narrowband interference and to frequency-selective fading due to multipath transmission thanks to the introduction of a guard interval in each symbol (also known as guard period or $G$ ). OFDM modulation and demodulation are based on fast Fourier transform (FFT) algorithms and it is commonly implemented in the digital domain, leading to cheap electronic devices with low complexity.

For these reasons, OFDM is the modulation of choice for a wide range of broadband digital communication standards, including, between others: the terrestrial digital video broadcasting (DVB-T) standard and wireless standards such as wireless LAN (WLAN) IEEE 802.11a and IEEE 802.11g, high-speed data connectivity IEEE 802.16e WiMAX [45], mobile communication 3GPP LTE and LTE-Advanced (also known as 4G), and the recent 3GPP 5G new-radio standard [131]. The characteristics of each standard are different in terms of the number of sub-carriers, symbol duration, guard interval, etc. It is particularly interesting to test the communication systems with full-standard signals instead of ad-hoc OFDM conventional signals, as the standard wireless signals include the physical layer (PHY) transport in which each sub-carrier is dedicated to data, pilot, guard, DC and null sub-carriers. For this reason, in our experiments, we use a vector signal generator (Keysight E4438C ESG) to deliver calibrated test signals following IEEE 802.16e WiMAX standard [45] specified with N7615B Signal Studio software. For more details regarding the experimental setup see Section 3.4.1. The WiMAX wireless signal, whose parameters are summarized in Appendix C, will be the one used in our frequency conversion performance. Noticeably, the ODFM modulation used in WiMAX is quite similar to the one in LTE for the downlink, so in principle the results shown here should also be applied to LTE systems.

In realistic systems, some extra information is added to the data to be transmitted. In our case, Binary Phase-Shift Keying (BPSK) modulated pilot tones are transmitted over the system for synchronization and control purposes in addition to raw data modulating other sub-carriers using M-QAM. As an example, a constellation diagram of a BPSK modulation is presented in Fig. 3.3(a), where the signal is displayed in a complex plane in which the horizontal axis states for the symbol components in phase with the optical carrier and in the vertical axis the components in quadrature. The horizontal and vertical axis are then labeled as phase (I) and quadrature (Q). Here, the points in this IQ scatter diagram depicts the modulated symbols at different sampling instants. In this case, as 
(a)

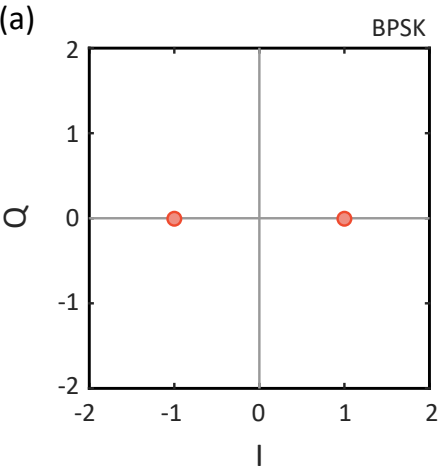

(b)

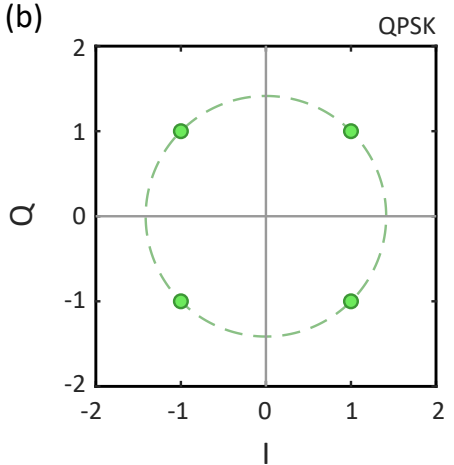

(c)

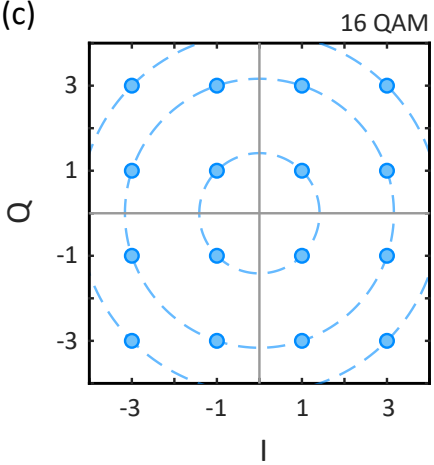

Figure 3.3: (a) Nominal QPSK IQ scatter diagram. (b) Nominal QPSK IQ constellation points arrenged around a equidistant circle (depicted in a green dashed line). (c) Ideal 16QAM IQ diagram showing 16 constellation points over different equidistant circles.

we are presenting a nominal BPSK constellation, all the points lie in the ideal locations thus resulting in no distortion of the signal. However, once the signal is transmitted over the system, noise and other impairments will cause that the actual constellation points deviate from that original locations. The measurement of the deviation or how far are the received points from the nominal IQ positions is the so-called error vector magnitude (EVM). This parameter will be used in the next sections for the characterization of the frequency conversion performance in our system.

Besides the use of BPSK modulated pilots, the data will be modulated in the different sub-carriers using either M-QAM or QPSK in its simplest form. The nominal constellation or IQ scatter diagram for a QPSK modulation is presented in Fig. 3.3(b). As can be seen from the picture, this modulation uses four constellation points that are equidistant around the dashed circle. This modulation per carrier is the one that will be used for most of our down- and upconversion experiments. However, also a 16QAM modulation, presented in Fig. 3.3(c), is also explored. In the last one, the carrier is modulated into 16 different amplitude and phase states, thus resulting in 16 constellation points (or 16 possible combinations of amplitude and phase of the sub-carriers).

\subsubsection{Downconversion using the first harmonic of the mechanical res- onance}

As mentioned above, the downconversion process is commonly employed at the receiver side of communication systems to decrease the frequency of the received data signal down to a band (IF) where information can be easily processed. To this end, in our system, the process is driven for an RF signal of frequency $\Omega_{R F}>\Omega_{m}$ when the cavity is set at the phonon lasing regime described in Chapter 2. It must be noted that only the first harmonic is considered in this configuration, but higher-order harmonics are also present and could be potentially used.

A schematic diagram of the proposed system using the OMC is presented in Fig. 3.4. Here, we can see the main elements involved in the process as well as the different optical (in blue) and electrical (in red) paths that the signals follow over the experiment. Bottom panels showing the frequency and bandwidth of the involved tones are also depicted at multiple points along with the system. Under these conditions, a tunable laser of frequency $\omega_{L}$ (Panel I) is modulated as described in the last section at a smaller 

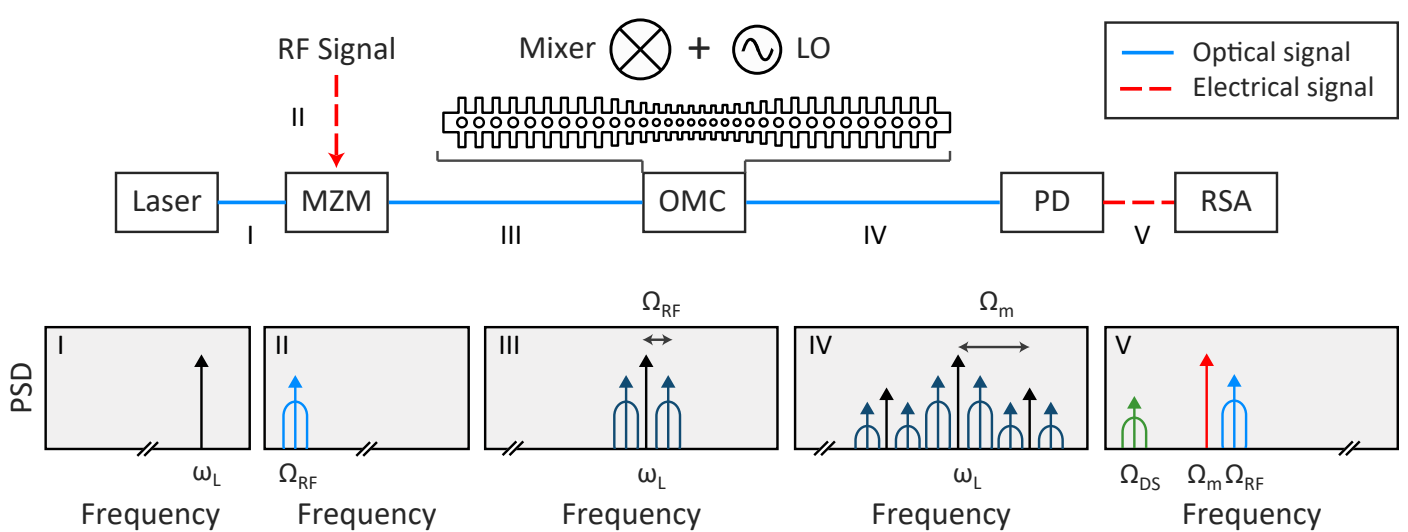

Figure 3.4: (Top) Schematic diagram of the frequency conversion proposed scheme where the OMC plays the role of both the LO and the mixer and (Bottom) their frequency signal representation at multiple points along with the system.

frequency than the oscillator's one (Panel II), thus resulting in a modulated optical field as in Panel III. Then, the modulated field is fed into the OMC under suitable conditions to drive the confined mechanical mode into the self-sustained oscillation regime. In contrast to the standard electrical frequency conversion scheme of Fig. 3.1, here the $\mathrm{OMC}$ has its own intrinsic LO whilst at the same time plays the role of the mixer. Once the signal goes through the cavity, it results in the generation of an OFC of OM nature, as described in Sec. 2.4. In this case, the optical spectrum output results in a series of peaks at frequencies $\omega_{L} \pm m \Omega_{m}$ with modulated sidebands (Panel IV). Finally, when the signal is photodetected we observe in the RF spectrum (only focusing in the first harmonic) the OM microwave tone at $\Omega_{m}$, the RF signal $\Omega_{R F}$ and the downconverted signal at the difference frequency $\Omega_{D S}=\Omega_{I F}=\Omega_{R F}-\Omega_{m}$, as depicted in Panel V.

In our experiment, the laser was modulated with an external microwave signal (the employed electrical power was $1 \mathrm{~mW}$ ) using an external Mach-Zehnder amplitude modulator. The external signal consisted of a pseudo-random sequence of data modulating a microwave signal (having a carrier frequency of $\Omega_{R F} / 2 \pi=3.9 \mathrm{GHz}>\Omega_{m} / 2 \pi$ ) using OFDM with QPSK modulation per subcarrier considering bandwidths spanning from $3.5 \mathrm{MHz}$ to $28 \mathrm{MHz}$, being fully compliant with the IEEE 802.16e standard [45]. The optical power reaching the cavity was $3.44 \mathrm{~mW}$ at $\lambda_{L}=1562.51 \mathrm{~nm}$, which was enough to drive the cavity into the phonon lasing regime. The measured RF spectrum of the downconverted signal is shown in the left panel of Fig. 3.5(a). The downconverted signal has been represented centered around its downconverted frequency $\left(\Omega_{D S} / 2 \pi \approx 50 \mathrm{MHz}\right)$ to better observe its $3.5 \mathrm{MHz}$ bandwidth. On the other side, the OM local oscillator frequency $\left(\Omega_{m}\right)$ and the original RF signal $\left(f_{R F}\right)$ are presented in the right panel of Fig. 3.5(a). By comparing the power of the detected RF signal when operating out of the phonon lasing regime $(\approx 28 \mu \mathrm{W})$ and the power of the down-converted signal when operating in the phonon lasing regime $(\approx 0.85 \mu \mathrm{W})$, the efficiency of the down-conversion process is estimated to be around $3 \%(\approx-15 \mathrm{~dB})$.

Figure 3.5(b) shows the evolution of the measured error vector magnitude (EVM) for both the RF and the down-converted signals as a function of the OFDM signal bandwidth. As expected, the EVM worsens as the signal bandwidth gets broader. However, 
(a)

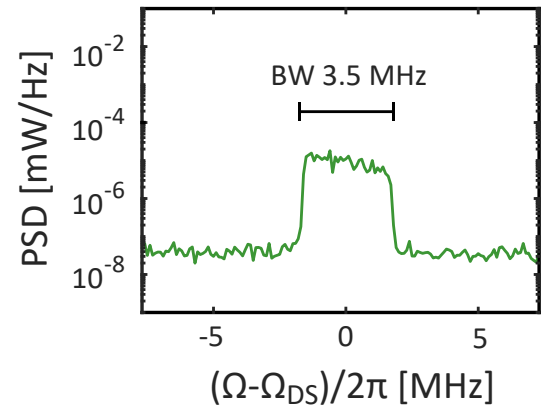

(b)

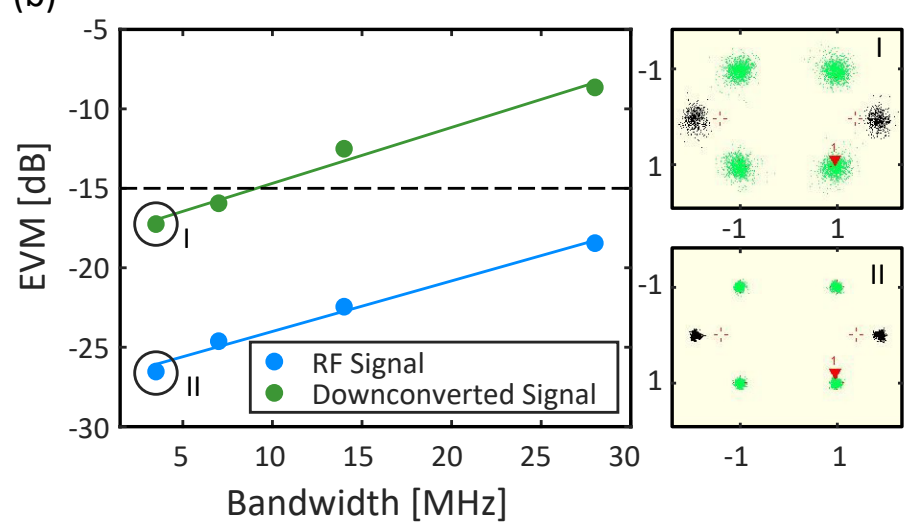

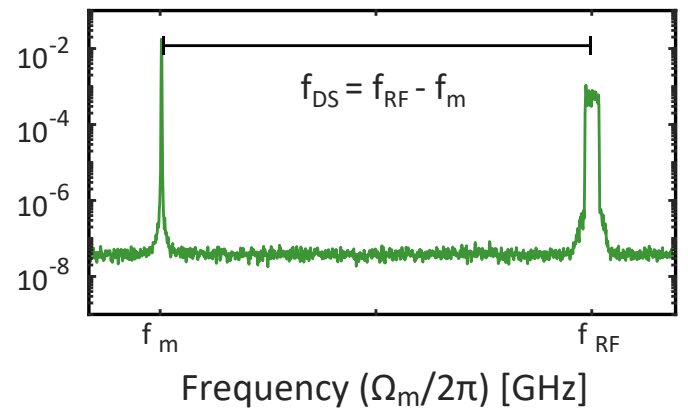

(c)

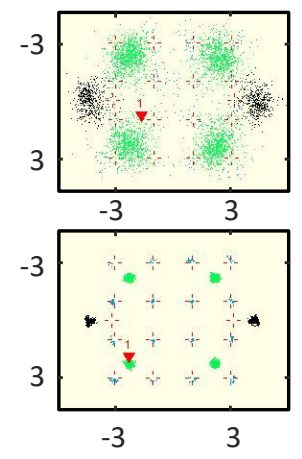

Figure 3.5: (a) RF spectrum of the downconversion process in the lasing regime. (b) Evolution of the EVM as a function of the bandwidth of the modulation for the input(blue) and the DC (green) signals. (Insets) QPSK Constellation for the DC signal in the top panel and the tone for the second panel. (c) Nominal 16QAM IQ diagram and 16QAM constellation for the DC signal in the right top panel and the tone for the second right panel.

a received $\mathrm{EVM}<-15 \mathrm{~dB}$, which is the threshold recommended at the received end in the standard [45], is obtained for signal bandwidths up to $7 \mathrm{MHz}$. In the inset panels, the received constellations for $3.5-\mathrm{MHz}$ bandwidth OFDM signals for both the RF and the down-converted signals are presented. The green points in the constellation correspond to detected data signals using QPSK modulation, the black points correspond to pilots using BPSK modulation. Remarkably, the recovery of QPSK data, as shown in the EVM measurements and the detected constellations, demonstrates that this technique is coherent since the phase is preserved in the frequency conversion process. In principle, improving the performance of the OMC would also allow satisfying the EVM threshold at higher bandwidths. It should be noted that this OM oscillator does not have any feedback loop which could increase the stability and improve the signal to noise ratio. Besides that, we also explored the possibility to downconvert a 16QAM-modulated OFDM signal. The resulting constellation for both the downconverted signal and the RF signal are presented in the top and lower panels in Fig. 3.5(c). In this case, we obtain values of the EVM of $-12.34 \mathrm{dBm}$ for a bandwidth of $3.5 \mathrm{MHz}$, thus not fulfilling the requirement. However, as already discussed, improving the signal to noise ratio (SNR) with the previously mentioned techniques should allow reaching lower EVM values. 


\subsubsection{Upconversion for higher order harmonics}

The OM cavity can also operate in reversely to the aforementioned case and perform as an all-optical frequency up-converter. As previously mentioned, frequency up-conversion is a process usually employed in communication systems and networks to increase the frequency of an incoming data stream up to a frequency band where it can be adequately radiated. To test the up-conversion process, the previous experiment is repeated employing actual OFDM data - again compliant with the IEEE 802.16e WiMAX standard - modulated at a lower frequency $\left(\Omega_{I F} / 2 \pi=f_{I F}=50 \mathrm{MHz}\right)$. Now, the key difference arises once the modulated field goes through the OMC. That can be seen from the photodetected signal in the configuration of Fig. 3.4 at Panel V is depicted in Fig. 3.6(a). At that point, multiple harmonics from the OFC generation appear modulated with lateral sibebands at $\Omega_{U S}=m \times \Omega_{m} \pm \Omega_{I F}$. The original signal at $\Omega_{I F}$ is also present at the lower part of the spectrum. However, it is noteworthy that these lateral sidebands could be tuned as a function of the input RF frequency signal. As an example, Fig. 3.6(b) shows the evolution of the lateral frequency sidebands (in green) around the different OFC harmonics (in red) for different frequencies of the original data signal. The evolution of the given IF signal (in blue) is present in the lower part of the spectrum.

(a)

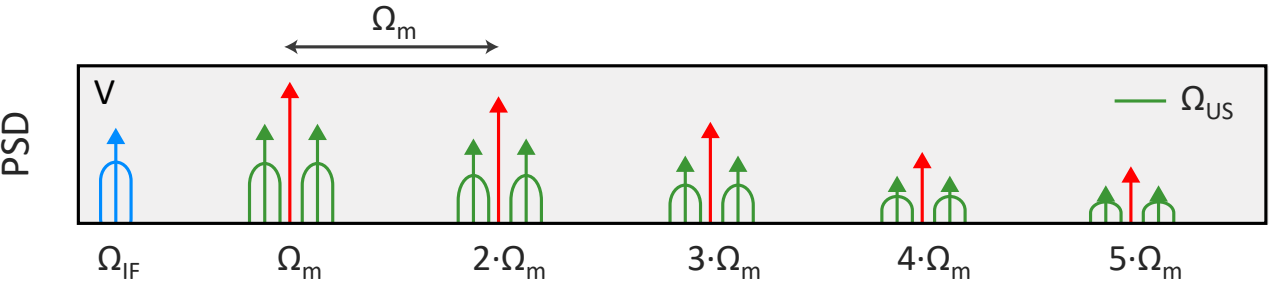

(b)

Frequency

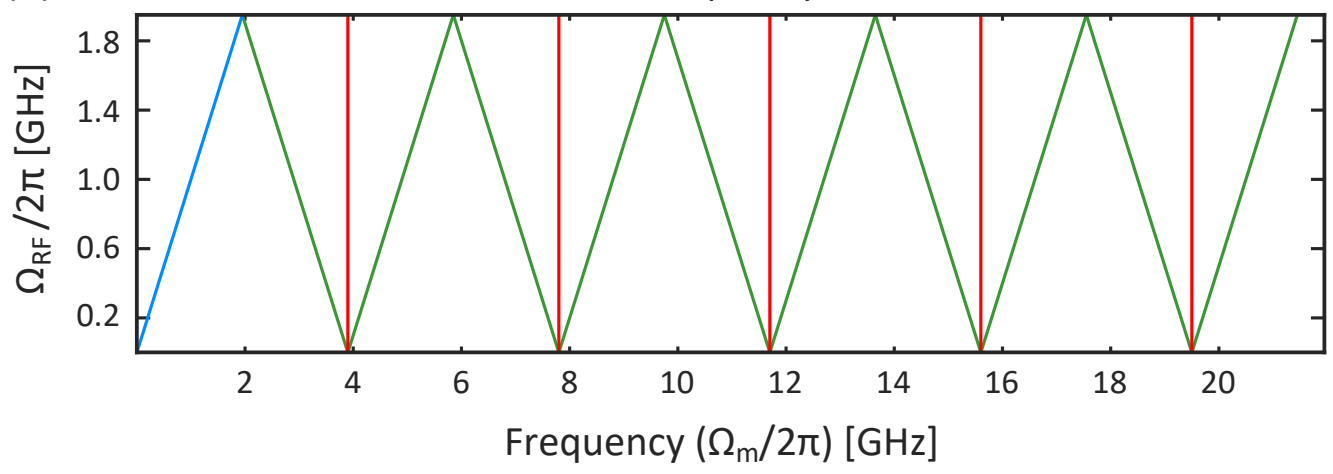

Figure 3.6: (a) Photodetected frequency signal representation for the frequency upconversion configuration. (b) Expected evolution of the upconverted lateral sidebands (in green) as a function of the IF data signal (in blue).

The detected electrical spectrum in reflection is depicted in Fig. 3.7(a), where lateral sidebands spaced $f_{I F}=50 \mathrm{MHz}$ from the different harmonics of the mechanical resonance can be observed as a result of the up-conversion process. The maximum observed frequency, corresponding to up-conversion using the third harmonic, is limited by the photodetector bandwidth $(12 \mathrm{GHz})$ used in these experiments. For more detail regarding the experimental setup see Section 3.4.1. This result suggests that this approach, which may use also cavities supporting mechanical resonances at other frequencies, may cover 
(a)

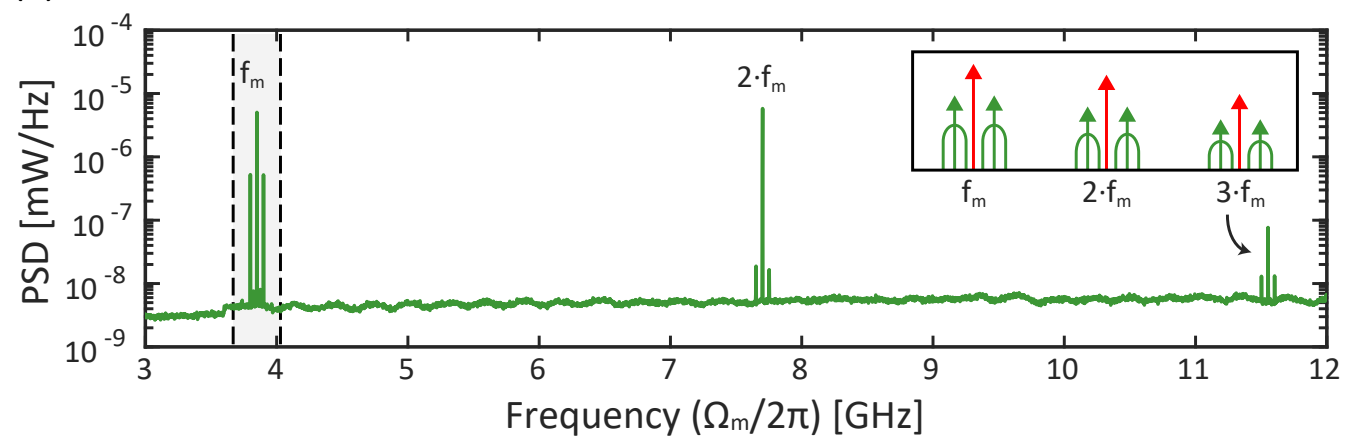

(b)

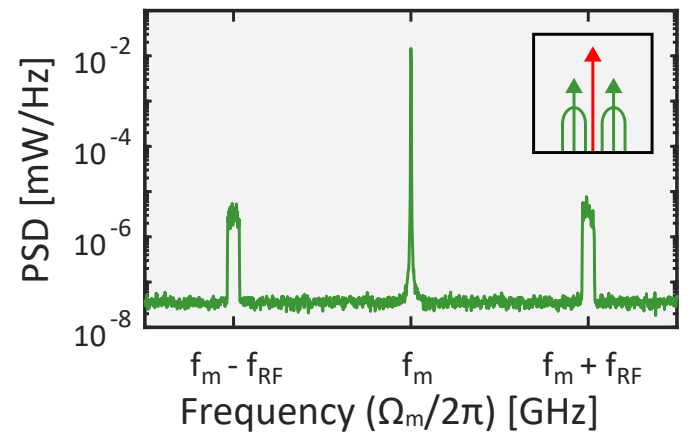

(c)

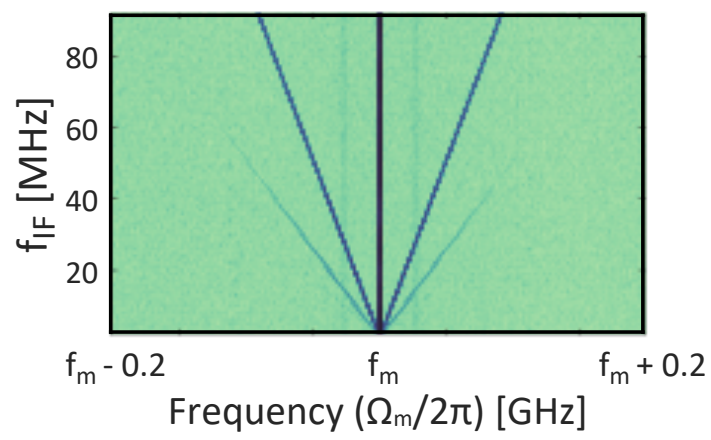

Figure 3.7: (a) Electrical spectrum of the upconversion process up to the third harmonic of the mechanical tone. (b) Close view of the upconverted RF signals sidebands around the first harmonic. (c) Evolution of the detected spectrum around the first harmonic as a function of the frequency of the modulation signal.

a wide range of microwave frequencies and, as a result, be used in multiple wireless systems. A close view of the lateral sidebands around the first harmonic is depicted in Fig. 3.7(b). Inset panels in the last figures are also shown illustrating the different frequency components of the signal.

In relation to the tunability of the system already introduced in Fig. 3.6. The experimental approach is shown in Fig. 3.8(c), where it can be seen the up-conversion process works for different values of the input frequency, although the nonlinearity of the cavity also results in the formation of frequency terms at $n \times f_{I F}$. In principle, we should be able to up-convert signals with frequencies up to $f_{m} / 2$ without overlapping with other sidebands of higher-order harmonics.

The measured EVM for the up-converted OFDM signals using QPSK subcarrier modulation is presented in Fig. 3.8(a) for the original IF signal as well as different upconverted harmonics. Even though the $-15 \mathrm{~dB}$ EVM recommendation at the receiver end is only met for the first harmonic (in this case in $f_{m} \approx 3.9 \mathrm{GHz}$ ), QPSK constellations can also be seen for the second and third harmonics (see insets). As above, improving the signal-to-noise ratio of the up-converted signal should enable accomplishing the requirement. Still, being able to recover the phase of the signal is a signature of the coherence of the up-conversion process, even for higher-order harmonics.

We also analyzed how the up-converted signal evolves as a function of the OFDM bandwidth for different signal bandwidths (from 3.5 MHz up to $28 \mathrm{MHz}$ according to [45]) for the first harmonic. As shown in Fig. 3.8(b), the results look similar to the case of 
(a)

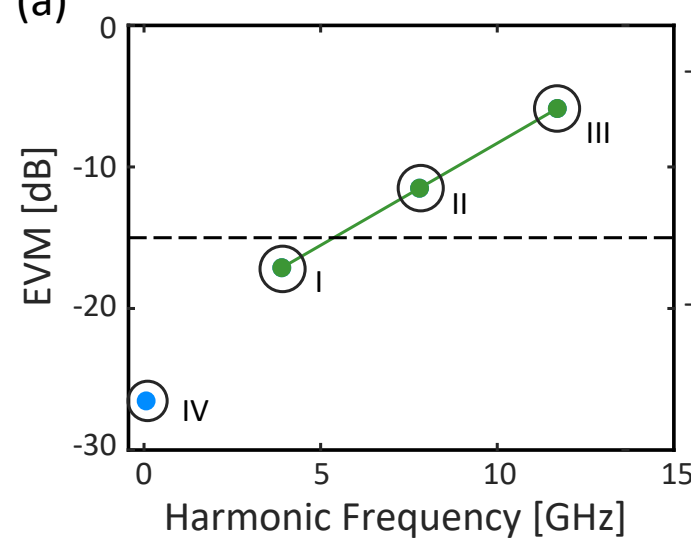

(b)
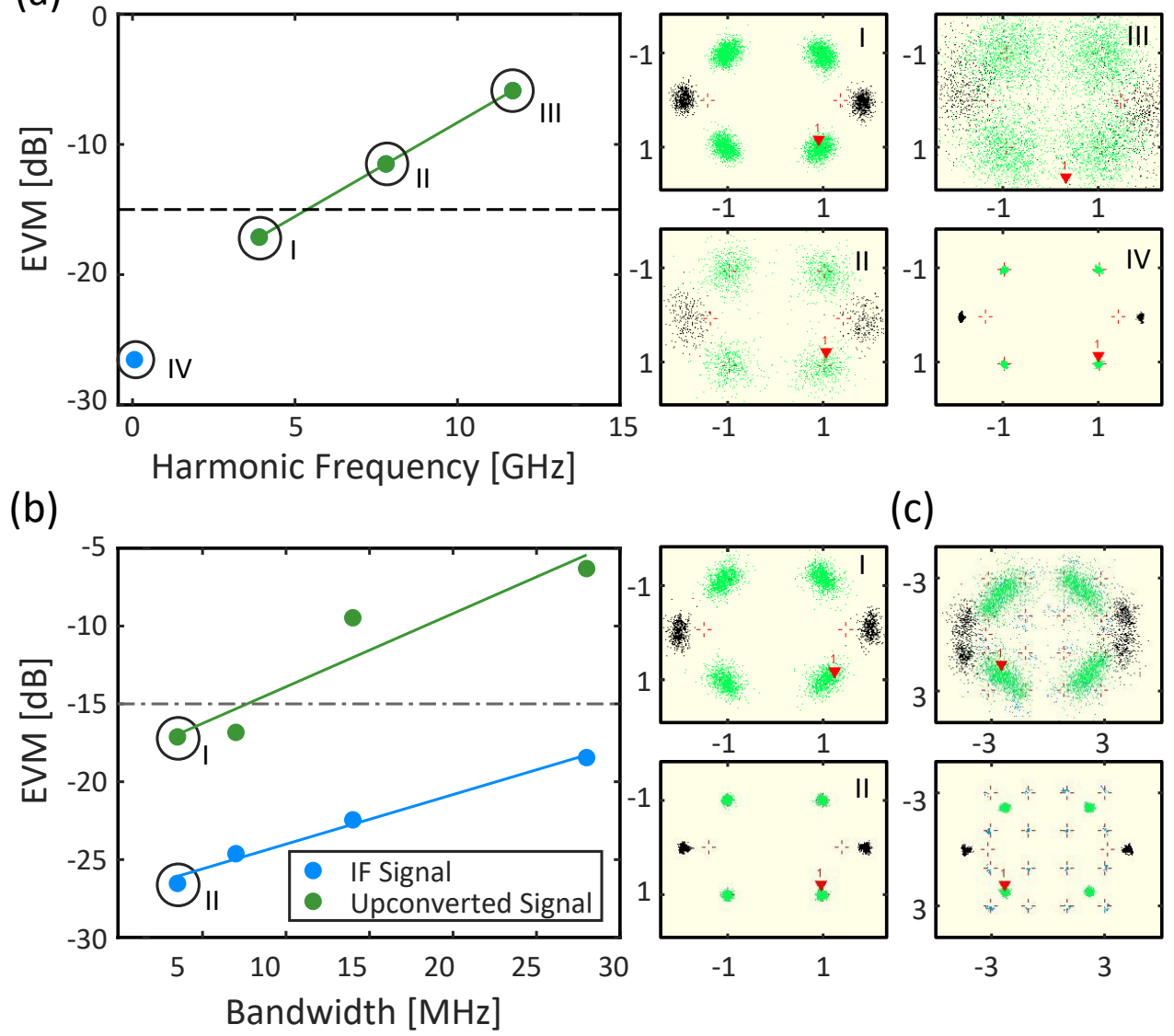

(c)

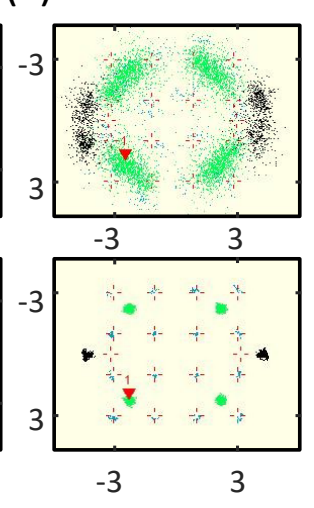

Figure 3.8: (a) Evolution of the EVM versus the harmonic used in the upconversion process. (Insets) Received QPSK constellations. (b) Measured EVM vs. OFDM signal bandwidth of the modulation signal (blue) and the 1st-harmonic upconverted signal (green). (Insets) Received QPSK constellations for the up-converted WiMAX signal at $f_{m} \approx 3.9 \mathrm{GHz}$ and at $f_{I F}=50 \mathrm{MHz}$. (c) $16 \mathrm{QAM}$ constellations for the 1st-harmonic (top) and the RF signal (bottom).

the down-conversion process: both $3.5 \mathrm{MHz}$ and $7 \mathrm{MHz}$ bandwidths satisfy the IEEE 802.16e EVM recommendation. This experimental result provides a proof-of-concept of the frequency conversion process for both down- and up-conversion applications with OFDM signals. In this case, the efficiency of the up-conversion process for the first harmonic is estimated to be around $2 \%(\approx-17 \mathrm{~dB})$ by comparing again the power of the detected RF signal presented above and the power of the up-converted signal when operating in the phonon lasing regime $(\approx 0.57 \mu \mathrm{W})$. Since in this case the up-converted power is distributed amongst different harmonics, it is reasonable to think that higher efficiencies in the first harmonic could be obtained by using cavities with larger Q factors so that higher-order harmonics are not generated. Lastly, in the case of a 16QAM modulation signal, the obtained constellations for the upconverted and the original RF signals, respectively, are presented in Fig. 3.8(c). 


\subsection{Experimental setup}

In this section, a modification of the experimental setup presented in Section 2.5.2 for the OFDM frequency conversion application measurements is described. The other measurements including phase noise and the mechanical mode evolution were obtained with the techniques described in Chapter 2.

\subsubsection{OFDM Frequency conversion setup}

As we already introduced, the OFDM frequency conversion setup, shown in Fig. 3.9, is a modification of the experimental scheme in Section 2.5.2. Here, the main difference is that the electro-optic modulator is an intensity modulator (labeled as MZM) set at the quadrature point to ensure a linear transfer response. Unlike in the calibration tone technique of Section 2.5.2, here the modulator is fed by an OFDM signal, with a tunable modulation frequency $\omega_{R F}$, via a vector signal generator (VSG) (Agilent E4438C ESG Vector Signal Generator). An example of the original OFDM signal created via the signal generator is presented in the left inset of Fig. 3.9.

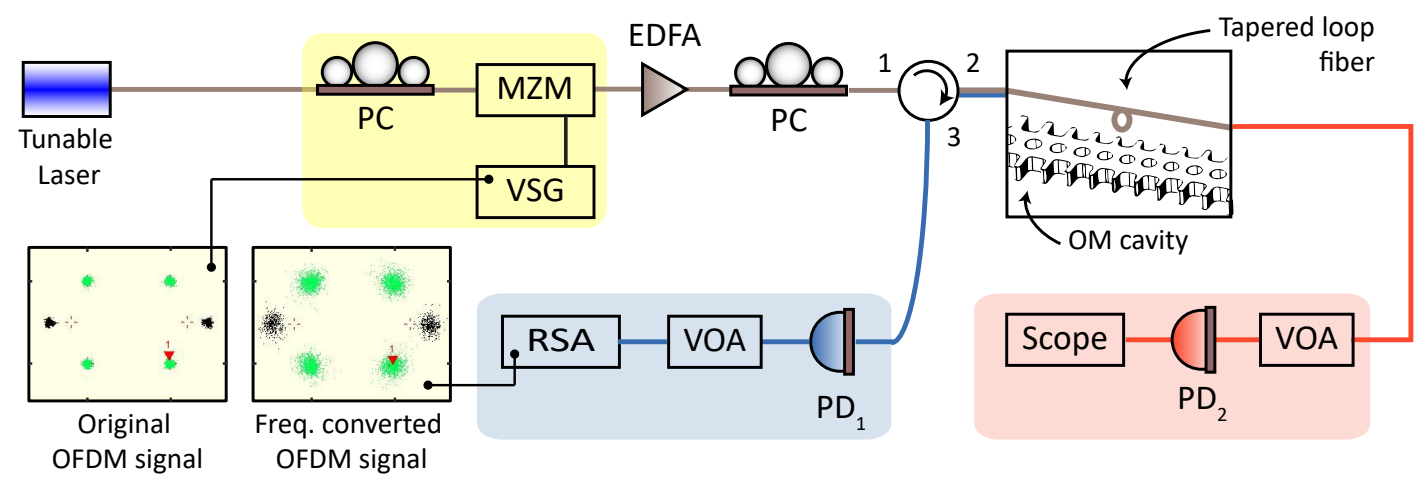

Figure 3.9: Experimental setup used in the characterization of the frequency upconversion process of an OFDM signal through an optomechanical crystal cavity. The inset figure shows a QPSK IQ diagram of the original and recovered OFDM signals at different points of the proposed experimental configuration.

Once the optical field has been modulated, it travels through the rest of the system as described in Section 2.5.1 until it reaches the OM cavity. Under coupling conditions, the cavity scatters light containing the mechanically-induced signal into both the reflection and transmission paths, so that this light can be properly measured. In this scheme, the transmission and reflection channels are used to measure the optical and mechanical response, respectively. Therefore, the reflection channel is where the measurement of the recovered OFDM signal is performed and analyzed with a Rohde\&Schwarz FSQ 40 Signal Analyzer through a WIMAX application firmware. By means of this firmware, the OFDM demodulation and error vector magnitude (EVM) analysis was performed. An exampled of a recovered OFDM QPSK signal is presented in the right inset of Fig. 3.9. Another RSA was also employed for the frequency trace evolution measurements involving the stability analysis (Aniritsu MS2850A Signal Analyzer). 


\section{Chapter 4}

\section{Multimode phonon lasing in an $\mathrm{OMC}$}

\section{Contents}
4.1 Introduction $\ldots \ldots \ldots \ldots \ldots \ldots \ldots \ldots \ldots \ldots$
4.2 Multiple mechanical mode confinement . . . . . . . . 48
4.2 .1 Phononic band diagram approach . . . . . . . . . . . . 49
4.2 .2 Individual single-mode phonon lasing . . . . . . . . . . . . 52

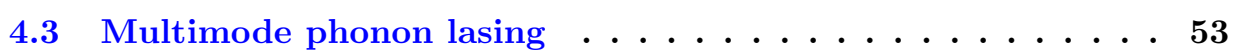

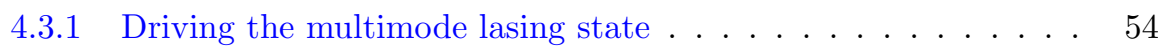
4.3 .2 Comparative stability analysis $\ldots \ldots \ldots \ldots \ldots$

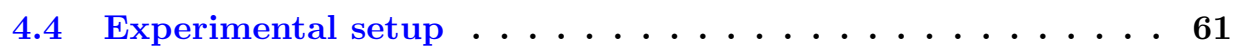
4.4.1 Multimode oscillation driving setup ........... 61

\subsection{Introduction}

In previous chapters, we have shown that silicon OM cavities can be relevant elements in microwave photonics and optical RF processing, particularly in applications requiring low-weight and compactness. In particular, such cavities can act as OM microwave oscillators in the phonon lasing regime (Chapter 2) and as frequency converters (Chapter 3). In the aforesaid chapters, however, only applications involving a single confined mechanical mode were addressed. Nevertheless, as also shown in Chapter 2, confinement of multiple mechanical modes can also be achieved in the OM cavities under study and their manipulation by means of radiation pressure effects may also be explored.

Indeed, even if multiple mechanical modes are coupled to a cavity, phonon lasing takes place for a single mode whose threshold condition is satisfied first, whilst the other modes get cooled [132] - similar to gain suppression (mode competition) in regular multimode lasers [133]. In optical lasers and parametric oscillators, mode-locking (or phase-locking) is achieved through temporal control techniques such as synchronous pumping [134]. Simultaneous phononic self-oscillation in multiple modes was observed in a low-finesse cavity, but without phase-locking [135]. A reliable route to phase-locked multiple phonon lasers would open the door to versatile OM-based signal synthesis, which is especially valuable at high GHz-frequencies in chip-scale devices. Moreover, it 
has broad significance in view of the emergent phenomena in multimode self-oscillating systems, including synchronization [47-53], stability enhancement [54], dynamical topological phases [55], and analog simulators [56].

In the present chapter, we explore experimentally the multiple mechanical mode confinement in a silicon OM crystal cavity with a total phononic bandgap, as in Chapter 2. Besides that, we show that, above threshold conditions, the phonon lasing regime can be achieved separately for different confined mechanical modes. Beyond this, we show that the single-mode lasing limitation can be overcome by using a Floquet approach in which the optical drive intensity is modulated in time. Recently, time-modulated radiation pressure was used to couple mechanical modes of different frequencies and enable mechanical state transfer [136], nonreciprocity [137], synthetic gauge fields [59], and entanglement [138]. Here, we show that a laser drive modulated at the difference between two mechanical oscillator frequencies can induce mode-locked coherent oscillation of both modes, as could be explained by establishing a Floquet theory. We observe this multimode lasing experimentally in a silicon OM crystal cavity supporting two GHz-frequency mechanical modes. We also find that the long-term stability of the output microwave tones are significantly improved in comparison to the single-mode oscillator, promising exploitation of these mechanisms towards highly stable, ultra-compact oscillators for microwave photonics.

\subsection{Multiple mechanical mode confinement}

As already outlined, multiple confined mechanical modes coupled through an optical field have been employed in the past years for several applications, including synchronization or stability enhancement. However, in most of these systems, the involved mechanical modes are not confined into the same physical structure. This is the case, for example, of confined mechanical modes in different crystal cavities which are coupled via a mechanical interaction [51] or coupled micromechanical oscillators which interact through an optical radiation field but are also physically located at different resonators $[48,54,139]$. Conversely, multiple mechanical modes confined into the same structure, interacting with one common intracavity field, have also been studied in the literature for a given number of oscillators $[59,140,141]$. However, they do not address a general route to multiple confinements of an arbitrary number of mechanical modes. Remarkably, most of these systems involve oscillators up to $\mathrm{MHz}$ frequencies, so a general route towards multiple $\mathrm{GHz}$ mechanical resonances in a same cavity is still missing.

The approach that we propose for multimode mechanical confinement is based on the OM crystal cavity presented in Chapter 2 . This cavity enables confining both a photonic and a phononic mode into the same structure. As a reminder, the mechanical mode was confined in a total phononic bandgap as experimentally depicted in Fig. 2.4(c), thus resulting in a reduction of the phonon leakage into the surroundings (a feature that should be appreciated at low temperatures where other losses mechanisms are avoided). The mechanical mode was mostly confined into the lateral corrugations of the structure, as could be appreciated both from the eigenvalues of the nominals unit cells (Fig. 2.2(d)) and the resulting mode in the final cavity (Fig. 2.3(b)). Nevertheless, previous chapters were focused only on the mechanical mode with the highest OM coupling rate, which was the one more easily transduced experimentally. However, other mechanical modes, located in other lateral corrugations, could also lie into the total bandgap, as can be seen from Fig. 4.1(a). Here, a PSD measurement of an OMC with six transition cells 

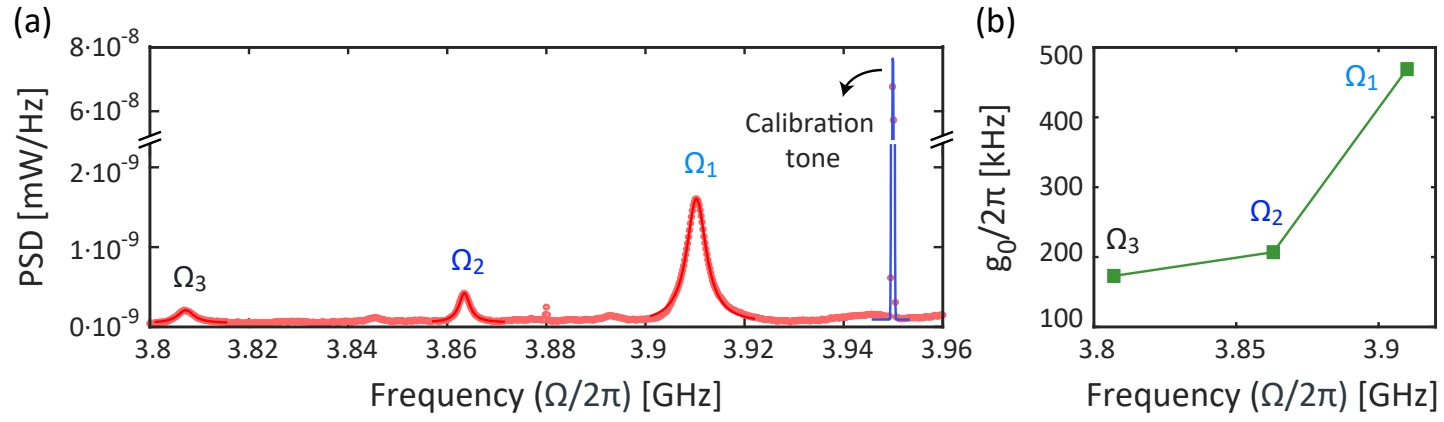

Figure 4.1: (a) Power spectra density of an OMC with 6 transition cells showing three confined mechanical modes (red) and a calibration tone (green) for the $g_{0}$ determination. (b) Evolution of the measured optomechanical coupling rate for a cavity containing three confined mechanical modes.

as the cavity presented in Chapter 2 is shown. It can be seen from the experimental measurement that three different mechanical modes can be distinguished at slightly different frequencies. The amplitude ratio between these oscillators can be understood through the analysis of their $g_{0}$, which was measured using the calibration tone technique reviewed in Section 2.5.2. From this analysis it can be seen that the highest frequency mode $\left(\Omega_{1}\right)$, as it is the one with the largest coupling rate, results in the one with highest transduced amplitude. On the other side, $\Omega_{2}$ and $\Omega_{3}$ result in a similar (and lower) coupling strength, as a result of a poorer transduction.

\subsubsection{Phononic band diagram approach}

In order to understand the confinements of multiple $\mathrm{GHz}$ mechanical modes in a single OMC and with the final aim of setting a route towards a general method, we can analyze the phononic band diagram as a function of the total number of transition cells $\left(\mathrm{N}_{T}\right)$ used to build up the cavity. This analysis is presented in Fig. 4.2, where the evolution of the confined mechanical mode band from the defect $\left(\mathrm{U}_{D}\right)$ to the mirror $\left(\mathrm{U}_{M}\right)$, at each constitutive cavity cell (depicted with dots), is shown. An overview of the structure with the labeled number of cells is shown in Fig. 4.2(b). For the cavity analyzed until now, consisting of $\mathrm{N}_{T}=6$ cells, the evolution of the possible confined mechanical mode is presented in the first panel of Fig. 4.2(a). Here, we can see that besides the confinement of the mode lying into the defect cell, the next two transition cells also result in confined mechanical modes inside the total bandgap (drawn with a shaded area). As the mode profile was located at the top and bottom corrugations, as in Fig. 2.2(d), this results in a picture where the different confined modes are always located at the wings (or corrugations) of the structure. As in the case of six transition cells, only three cells (the defect one and the next transition cells) lie into the phononic bandgap, at best three mechanical modes will be present in the final structure, as shown in Fig. 4.1.

Consequently, an OM crystal cavity created with more transition cells should result in more confined mechanical modes (or mechanical resonances), as can be seen in the second and third panels in Fig. 4.2(a), for $\mathrm{N}_{T}=9$ and 12 respectively. A summary of the evolution of the total number of confined modes into the total bandgap is presented in Fig. 4.2(c). Here, the different mechanical modes as a function of $\mathrm{N}_{T}$ are presented 
over the frequency span of the total bandgap. As higher are the number of transition cells the total amount of localized mechanical oscillators that we can achieve is increased. Furthermore, the mechanical frequencies get closer to each other because if more elements shape the cavity, the physical dimensions between successive cells remain closer.

(a)

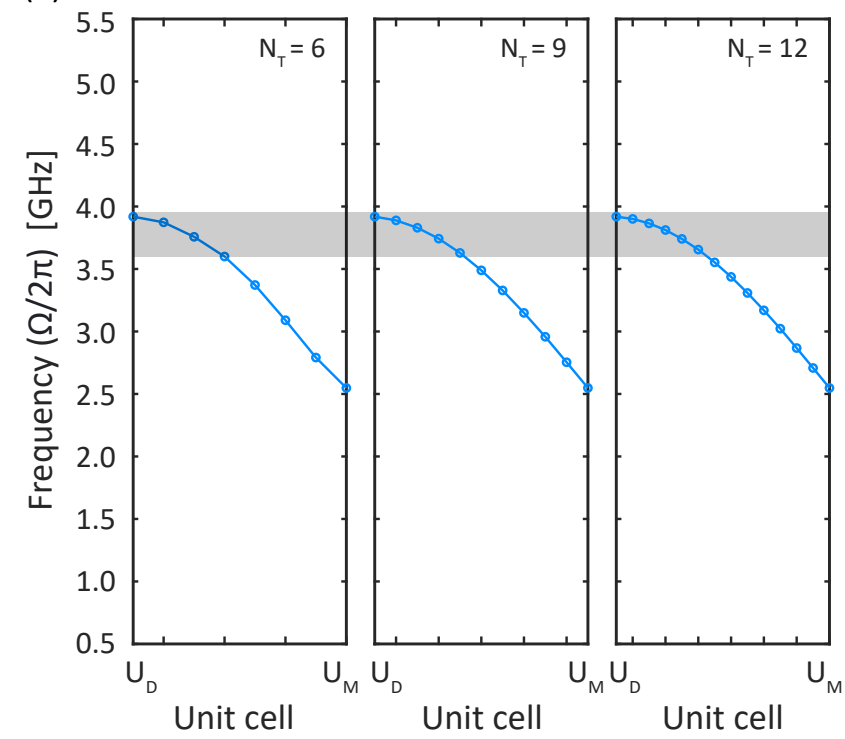

(b)

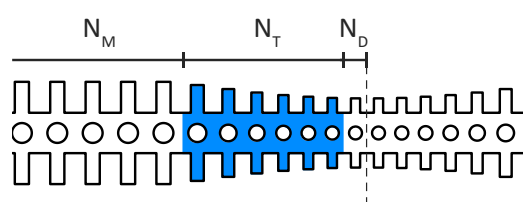

(c)

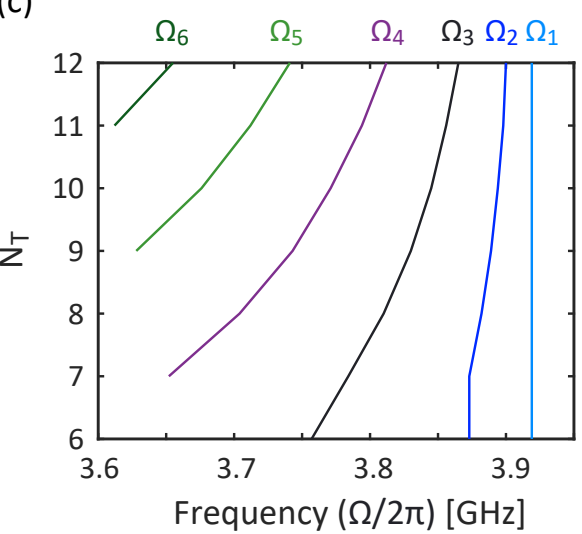

Figure 4.2: (a) Phononic band diagram evolution of the confined mode band for different transition cells. (b) Close view of the transition cells of the OM cavity. (c) Frequency evolution of the confined mechanical modes as a function of the number of transition cells.

(a)
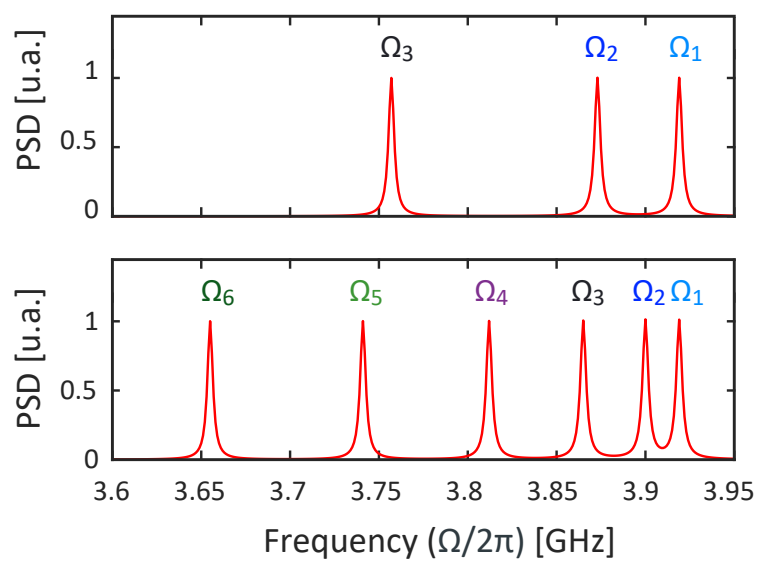

(b)
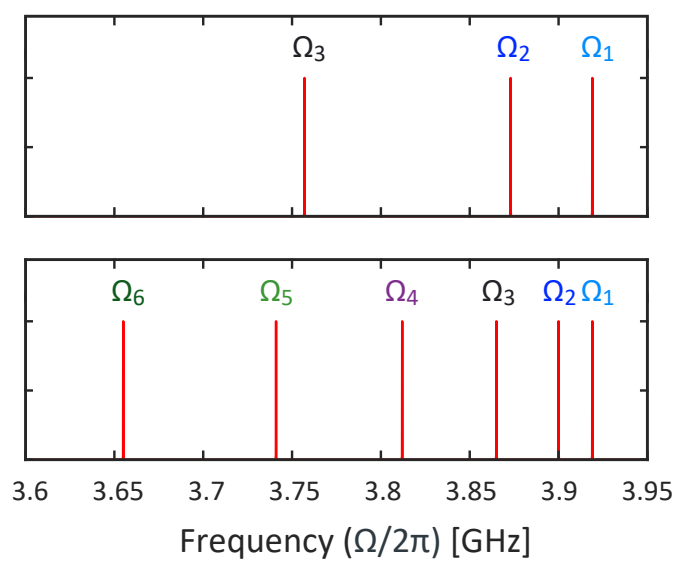

Figure 4.3: (a) Expected PSD confined modes for a transition cell of $N_{T}=6$ (top) and $N_{T}=12$ (bottom) with mechanical quality factors $\mathrm{Q}=1000$. (b) Expected PSD confined modes with mechanical quality factors of $\mathrm{Q}=10^{5}$.

Assuming a constant OM coupling rate for all the confined modes and a mechanical 
quality factor of 1000, in agreement with the values presented in Fig. 2.5, the transduced mechanical spectrum for an OM crystal cavity with 6 and 12 unit cells will result in the panels of Fig. 4.3(a). The mechanical $\mathrm{Q}=10^{3}$ is the common estimation for room temperature situations [81], meaning that the mechanical damping is limited by material losses [142]. Upon this assumption, the top picture resembles the experimental measurement already presented in Fig. 4.1 where the difference in the peak amplitude was related to the actual OM coupling rate. Regarding the oscillator's frequencies, they can differ from the nominal ones because of fabrication imperfections, which will be thoroughly analyzed in the next section.

It should be noted that this general method could be limited up to the peak resolution in order to distinguish different mechanical modes. This can be already seen for the $\mathrm{N}_{T}=12$ cavity in the bottom of Fig. 4.3(a). In this case, as the mechanical frequencies approach each other, $\Omega_{1}$ and $\Omega_{2}$ start to overlap, but still remain resolved. The limitation is thus overcome when the frequency difference between two modes is less than full width at half maximum. However, as this limitation is related to the broadening of the peak, it can be overcome at low temperatures where the mechanical quality factor can become extremely large [143]. Therefore, a resulting transduced spectrum of these OMC will eventually result in the panels presented in Fig. 4.3(b) for a nominal case of $\mathrm{Q}=10^{5}$.

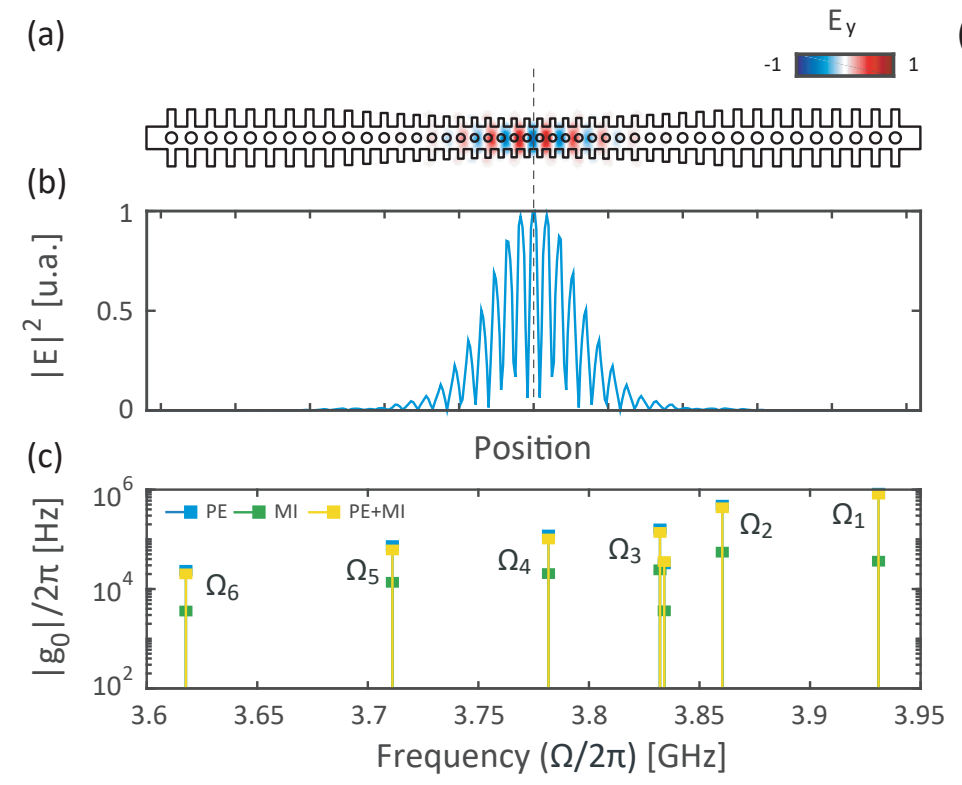

(d)

\section{$\Omega_{1}$}

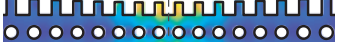
0000000000000000

$\Omega_{2}$

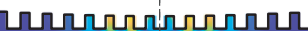

000000000000000

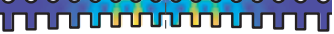

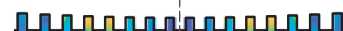

$\Omega_{3} 0000000000000000$

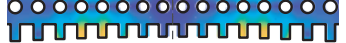

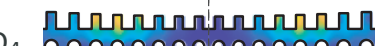

40000000000000000

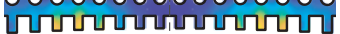

$\Omega_{5}$

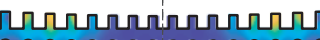

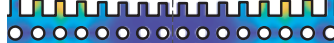
نோฯ

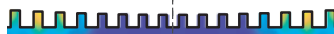

0000000000000000

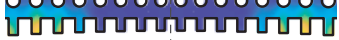

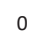

u

Figure 4.4: (a) Optical field profile for an OMC with 12 transition unit cells. (b) Normalized total electric field variation along the total length of the cavity. (c) Calculated OM coupling rate contributions. (d) Phononic field profile for the mechanical modes giving the highest $g_{0}$.

Concerning the peak amplitudes in Fig. 4.3, they were considered to be equal as, for the sake of simplicity, we assumed that all the modes had the same OM coupling rate. However, the OM coupling strongly depends on the overlap between the photonic and the phononic field profile. As the electric field profile of the photonic mode decreases towards the cavity extremes, it may be expected that $g_{0}$ will also be reduced for lower mechanical frequency modes, which are less confined in the central region. This can be 
seen in Fig. 4.4(a,b) where the optical field profile and its evolution along the length of the cavity are presented for an $\mathrm{OMC}$ with $\mathrm{N}_{T}=12$. It can be appreciated that, as already stated, the normalized total electric field $\left(|E|^{2}\right)$ drops towards the lateral cavity sides and it reaches its maximum at the middle of the structure. Therefore, the more centered a mechanical mode in respect to the cavity, the higher coupling with the optical field profile will experience. This can be checked out in Fig. 4.4(c), where the total OM coupling rate as well as their PE and MI contributions are presented for the different confined modes. It must be noted that the $\mathrm{PE}$ and $\mathrm{MI}$ effects have opposite contributions, thus resulting in a slightly lowered total AO interaction.

The resulting total displacement of the different confined mechanical mode profiles is presented in Fig. 4.4(d). Here, we can see that the mechanical profile is mainly located at the lateral corrugations of each unit cell. Indeed, the profile of the displacement looks quite similar for all the modes. The main difference is that as we move from the cavity center, the wings become longer, which reduces the mechanical frequency proportionally.

\subsubsection{Individual single-mode phonon lasing}

As discussed in the last section, multiple mechanical mode confinement can be achieved via the engineering of the unit cell to shift adiabatically the modes of the phononic band diagram. Once these mechanical oscillators are confined into the same structure, an analysis of the possibility of driving them into the self-oscillating regime is needed in order to take benefit of all the applications explored in Chapters 2 and 3. Thus, in order to explore a system displaying individual phonon lasing of different mechanical modes, we propose a system where a single optical radiation field interacts by means of radiation pressure with two confined mechanical modes, as suggested in the scheme of Fig. 4.5(a). Specifically, we use the 1D OM crystal cavity with six transition unit cells discussed along with this thesis. This cavity has a high-Q optical mode and two tightly localized mechanical modes. The transduced mechanical spectra of those oscillators are presented in Fig. 4.5(b). Notably, the OM coupling rate and damping of both mechanical modes are quite similar, which also leads to a similar cooperativity $\left.C_{j}=4 g_{j}^{2}\left|\bar{\alpha}_{0}\right|^{2} /\left(\Gamma_{j} \kappa\right)\right)$ : $C_{1} /\left|\bar{\alpha}_{0}\right|^{2}=(5.6 \pm 0.4) \times 10^{-5}$ for mode the mechanical mode $\Omega_{1}$, also labeled as P1, and $C_{2} /\left|\bar{\alpha}_{0}\right|^{2}=(3.7 \pm 0.3) \times 10^{-5}$ for mode the mechanical mode $\Omega_{2}$, labeled as P2.

As it has been pointed out in previous chapters, the final structures usually differ from the nominal ones because of fabrication imperfections. In order to ensure that the measured mechanical modes were still located in the middle region of the cavity, a calculation of the mechanical modes was performed using a profile retrieved from a SEM image. In Fig. 4.5(c) both the theoretical mechanical mode profile of the nominal and the retrieved fabricated structures are compared. Here, we can see the similarity between the two profiles of the mechanical modes in both cases.

Noticeably, because of the similarity in terms of coupling rate and cooperativity, we observe that either mode can be individually driven to a self-sustained oscillation (SSO) state under blue-detuned driving, as shown in Fig. 4.6(a) for P2 and in Fig. 4.6(b) for $\mathrm{P} 1$. Interestingly, if any modulation is set into the system and we let them freely evolve, as is the case in the presented experiment, only P1 or P2 reach the self-oscillating state. This is related to the fact that, for a multimode mechanical system, it has been proved that the self-sustained oscillation, also addressed as phonon lasers, takes place just only for a single mode, the one whose threshold condition is satisfied first. This is the same as for conventional optical lasers, where a saturation phenomenon enables the lasing of 
(a)

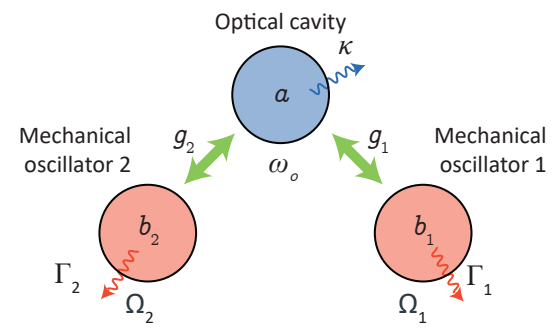

(b)

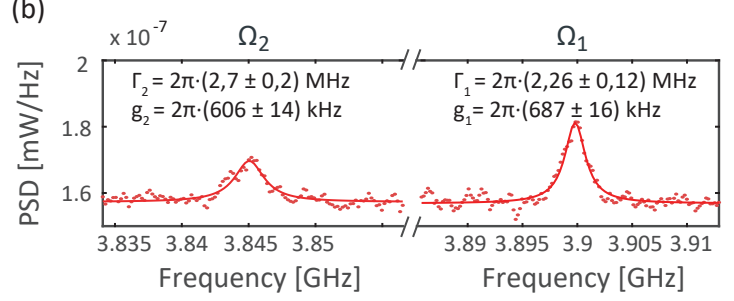

(c)
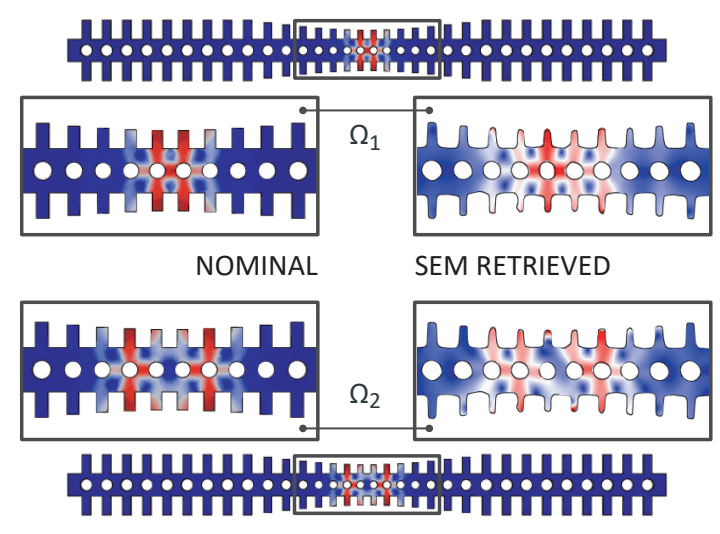

Figure 4.5: (a) OM interaction scheme of a common optical field interacting with two free mechanical oscillators. (b) Thermally transduced mechanical spectra of the two confined mechanical modes into the same structure. (c) Comparison of the total displacement profile of the nominal cavity under study and the real SEM retrieved profile.

one mode whilst suppress the gain for the others [133].

Mode competition is also present in phonon lasers in OMCs: it has been already demonstrated that once a mechanical mode is set into the self oscillated regime, the others get damped [132]. In fact, this is something that we also observe when either there is no modulated input light field into our system or if it is modulated at an arbitrary modulation frequency not equal to the difference frequency between the involved mechanical modes. This can be seen from Fig. 4.6(c), which shows the amplitude evolution of the two mechanical modes when an input laser is swept across the resonance in the blue-detuned regime. A close view of the amplification of the modes is outlined in the inset of Fig. 4.6(c). Here, we can see that at first, the two mechanical modes start getting amplified and shift in frequency (region A), as in the case of a single oscillator [67]. However, once one of the modes reaches the instability threshold condition (P1 in this particular case), that mode is then further amplified and finally reaches the phonon lasing condition and meanwhile the other (P2) gets cooled (region B), evidencing gain suppression. Finally, once one of the modes has reached the phonon lasing condition, it will stay oscillating until the laser exits the cavity (region C). Despite here we have only presented the case for P1 reaching the SSO state and P2 being cooled, the opposite situation behaves in the same way. In fact, the final state of the system with one or the other into the SSO regime strongly depends on thermal fluctuations [132].

\subsection{Multimode phonon lasing}

To overcome the single oscillating mode limitation already discussed, different approaches can be followed: a "spatial" variation of the optical field degree of freedom [139] or temporal variation of the pumping field, which is the technique that we explore here. A schematic scheme of the modified system already discussed is presented in Fig. 4.7. Here, the objective is to commonly set to oscillate the two mechanical modes at their 
(a)

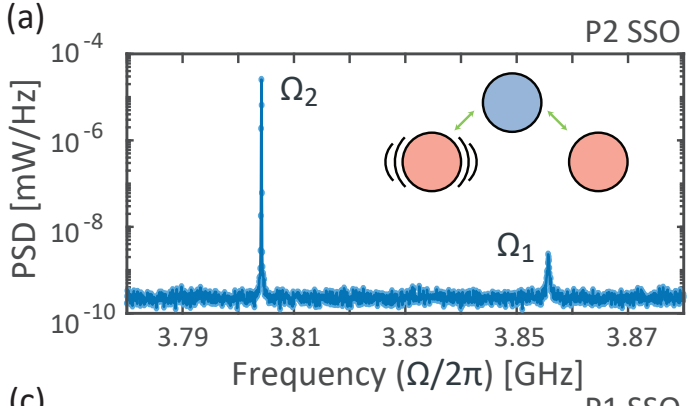

(b)

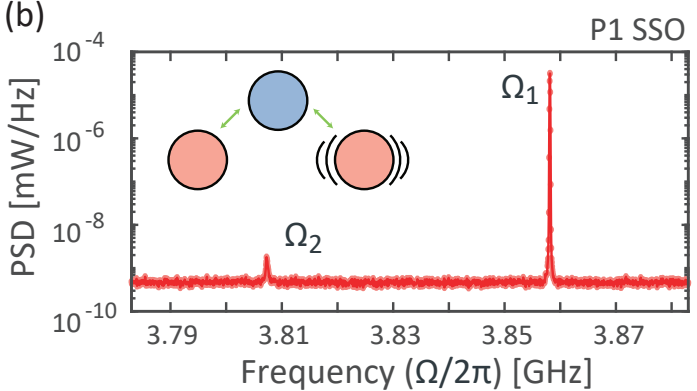

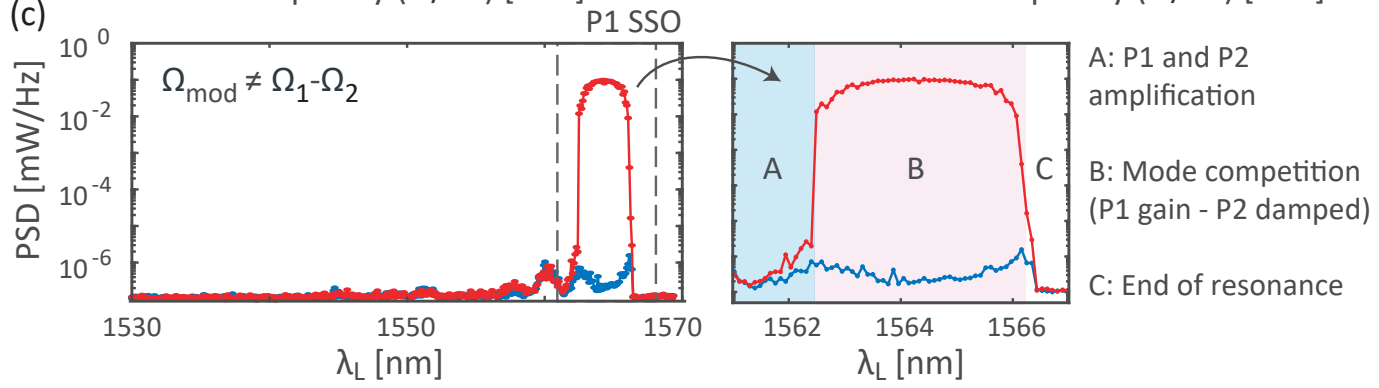

Figure 4.6: (a) Self-oscillating spectra of the second mechanical mode P2. (b) Selfoscillating spectra of the first mechanical mode P1. (c) Evolution of the system until the SSO excitation of P1 through a wavelengths scan with a blue detuned laser with no modulation input field or at a modulation frequency $\Omega_{\text {mod }}=\Omega_{1}-\Omega_{2}$. The input power in this experiment was set at $P_{i n}=3.16 \mathrm{~mW}$.

own oscillator frequencies by means of a modulation of the input light field. Nonetheless, as it will be discussed broadly in the next chapter, the frequency of the modulating signal must have a required value.

\subsubsection{Driving the multimode lasing state}

With the aim of driving the two mechanical modes into the self-oscillating regime, thus overcoming the single phonon lasing limitation, a modulated input field is set into the cavity. However, it must be noted that both the modulation frequency as well as how the cavity is driven have an influence on the final state of the system. In order to discuss the driving procedure, we have explored two different configurations. In the first configuration, the system was driven first reaching the SSO state for P1 or P2 and then setting on the modulation frequency at the difference frequency between the two involved mechanical modes. Moreover, a scan over different modulating tone frequencies was also considered. The results of such experiments are presented in Fig. 4.8.

Once P1 or P2 is lasing, an upconversion of the drive modulation tone occurs, similar to what happened in the previous chapter. This means that the drive tone gives rise to a lower and an upper sideband of the lasing tone in the optical modulation spectrum. This can be seen in Fig. 4.8(a), which shows many spectra (colored from black to red) that are taken while varying the modulation frequency around the difference frequency of $\mathrm{P} 1$ and P2. A close view of this scan is shown in Fig. 4.8(b). Here, we can see that for modulation frequencies around $\Omega_{1}-\Omega_{2}$, the lower sideband (LS) overlaps with the mechanical mode P2. To observe whether the mechanical modes P2 or P1 experience a change, we analyzed the amplitude of those modes and the lower sideband. It has to 


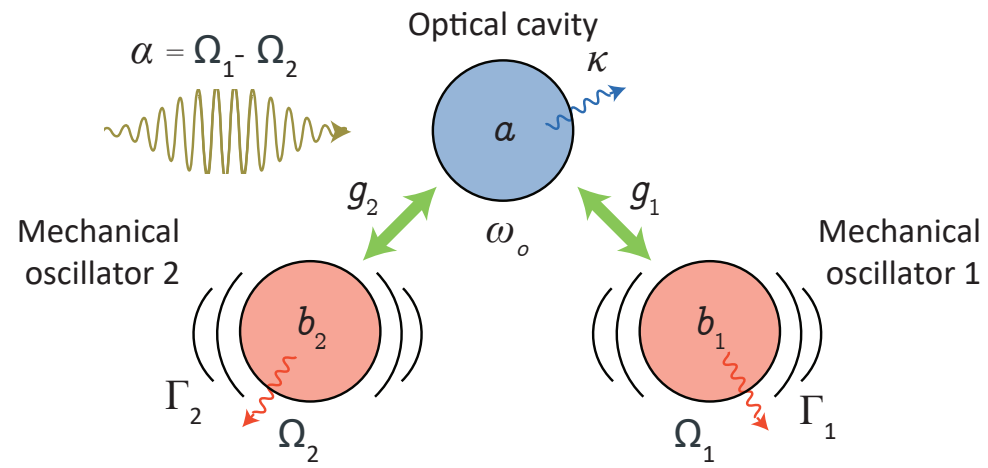

Figure 4.7: $\mathrm{OM}$ interaction scheme of a common optical field driven by a modulated signal interacting with two free mechanical oscillators.

be noted that in the case of the overlap between $\mathrm{P} 1$ and the lower sideband, we have performed a fit of a Lorentzian shape in the case of the thermally driven mechanical mode P1 and a fit of a Gaussian shape in the case of the lower sideband driven by modulation tone as can be seen in Fig. 4.8(c). We find that the linewidth of the Lorentzian (P1) is never narrowed while the modulation sideband of $\mathrm{P} 2$ is scanned in and out of resonance with mode P1. This demonstrated that the MMO state cannot be reached from the SSO state and the narrow tone observed in the spectrum comes from the up-conversion of the modulation signal. The resulting evolution of both P1, P2 and LS amplitudes are presented in Fig. 4.8(d).

Based on this analysis, we have seen that starting from P1 in a SSO state and stepping the modulation frequency across resonance $\Omega_{\text {mod }}=\Omega_{1}-\Omega_{2}$ does not activate the MMO state, as the thermomechanical Lorentzian of P2 remains unaffected (see Fig. 4.8(d)). Instead, the MMO regime is reached when the intermodal coupling is established before, allowing the Floquet modes to cross threshold simultaneously [144]. We experimentally achieve this by setting the modulation on, fixed to the measured difference frequency, and then sweeping a far-blue-detuned laser frequency towards the cavity resonance. Therefore, to reach the MMO regime the experiment was performed as follows: First, the difference frequency between the two involved mechanical modes was characterized at different laser wavelengths. Next, this difference frequency was set as the modulation frequency of the laser driving the cavity $\Omega_{1}-\Omega_{2}$, and it was kept constant in the rest of the experiment. Indeed, we modulated the laser intensity at frequency $\Omega_{\text {mod }}$ and a modulation depth of $d=0.18$. Finally, a sweep of the laser wavelength on the blue-detuned side of the resonance was performed.

In this experiment, in addition to the measurement of the final state of P1 and P2, we analyzed the difference tone created by these two signals employing an external mixer set after photodetection. This also allows us to determine if the two tones are mode-locked, which will happen if the difference tone is ultranarrow. Despite all the experimental details are presented in Section 4.4.1, an overview of the proposed measurement scheme is presented in Fig. 4.9(a). Here, we have also presented the expected PSD signal at different points along with the system. The main idea is that, in order to ensure that the measure the difference beat note and that this signal is not superposed to the original RF modulated frequency, we filter the last one previous to the mixing process. As can be seen in Panel III, once we perform the experiment driving the modulation into the 
(a)

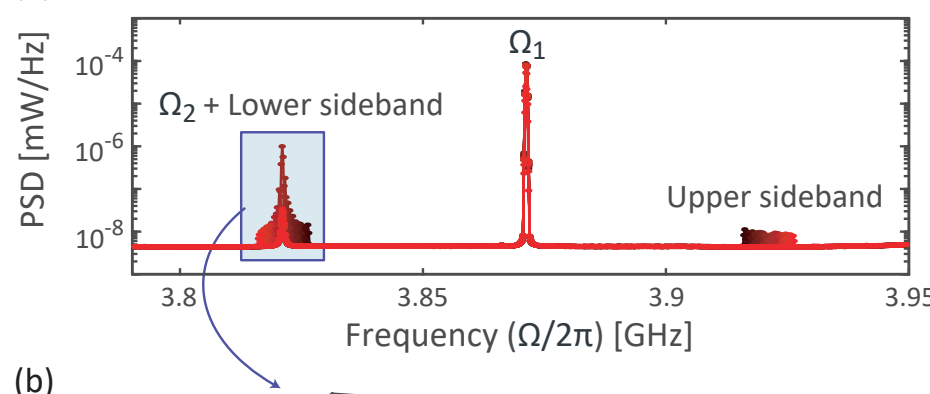

(b)

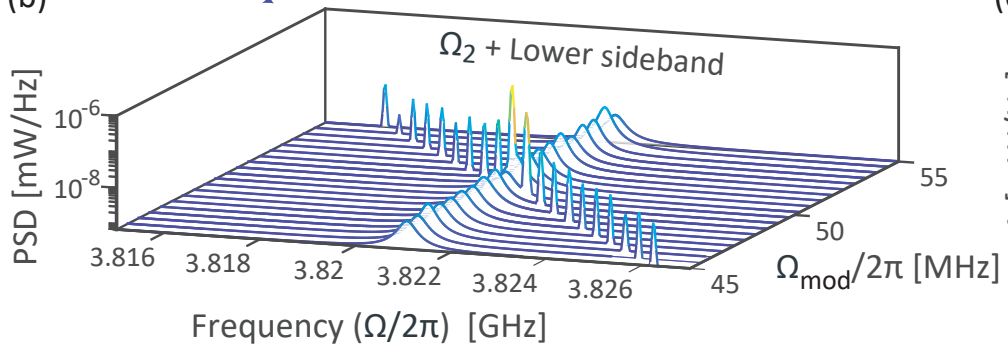

(c)

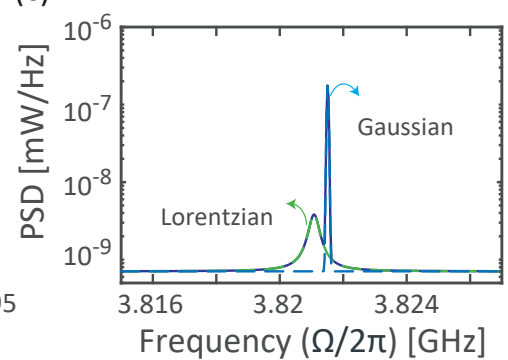

(d)

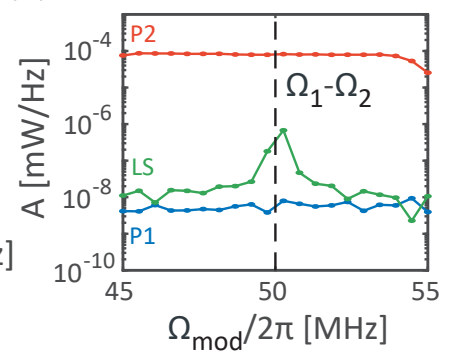

Figure 4.8: (a) Overview of the upconversion of the modulation tone around $\Omega_{2}-\Omega_{1}$. (b) Close view of the scan of the modulation tone around P2. (c) Example of the different fit contributions in the overlap between P2 and the lower sideband. (d) Evolution of the amplitudes of P1, P2 and the lower sideband as a function of the modulation frequency tone.

system previously to the sweep of the laser, the detected signal consists then in the two multimode self-oscillating modes as well as the original modulation tone. However, through a bandpass filter, we can get rid of this modulated signal, as depicted in Panel IV. Finally, once we divide and mix the filtered signal, the resulting spectra will contain both the multimode phonon lasers as well as the difference tone where no other remaining signal can overlap with it.

To conclude, the resulting multimode phonon lasing state is shown Fig. 4.9(c,d). Figure 4.9(c) shows the difference beatnote of the two lasing tones, measured after passing the signal through a splitter and microwave mixer. Its narrow linewidth, much smaller than the linewidths of the individual tones and only limited by the $1 \mathrm{~Hz}$ resolution bandwidth of the spectrum analyzer, confirms the phase or mode-locking of the two lasing phonon modes. This measurement was performed at a frequency span of $20 \mathrm{~Hz}$ and a resolution bandwidth of $1 \mathrm{~Hz}$ but it was limited by the Gaussian filter of the real spectrum analyzer used in our experiments. On the other hand, Fig. 4.9(d) shows the obtained two multimode mechanical modes that are self-oscillating at their oscillator frequency.

It must be noted that despite the system is considered to be mode-locked, it is not synchronized. Synchronization is a physical phenomenon that happens when two or more oscillatory and independent systems, which are not isolated from their environment, spontaneously lock to a common frequency. However, besides that, other conditions may also be fulfilled. Briefly, first, the individual oscillators need to be in a self-sustained oscillation regime. Second, the systems may adjust their frequencies by means of weak coupling, and finally, the synchronization should only happen when the frequencies of the 
(a)

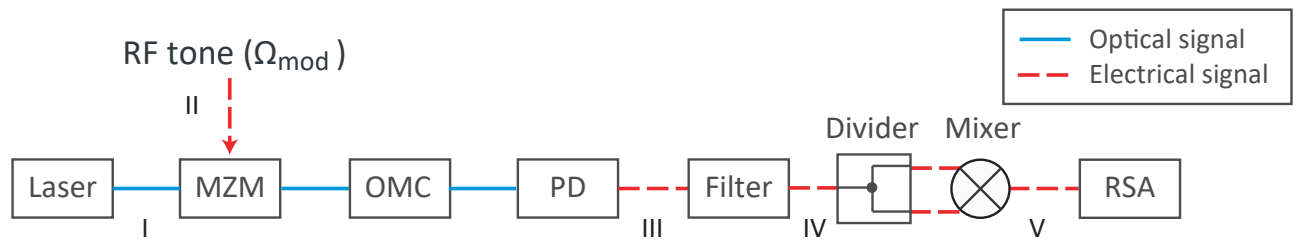

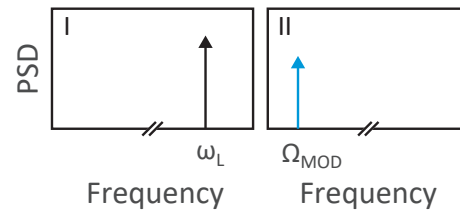

(c)

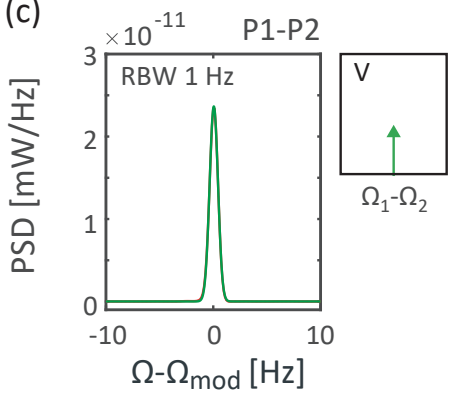

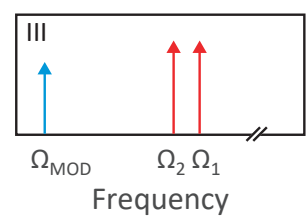

(d)

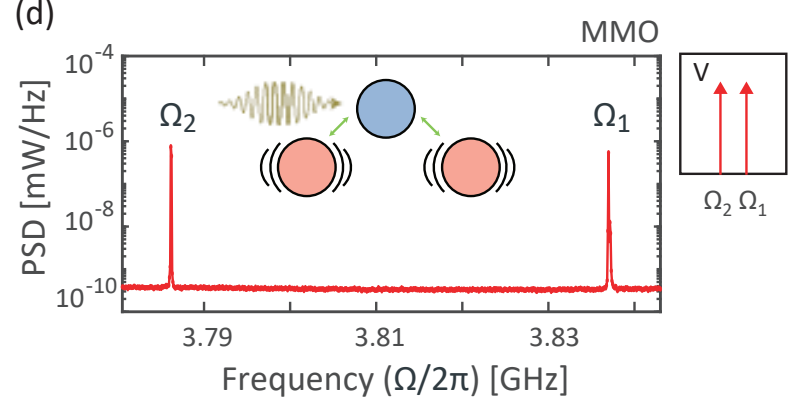

Figure 4.9: (a) Schematic diagram of the experimental setup showing the frequency signal representation at different points along the system. (b) Difference tone obtained by mixing the multimode phonon lasers in the microwave domain. (c) Spectrum of the two mechanical oscillators when lasing simultaneously at the MMO state.

involved oscillators are close enough to each other [145]. However, in our work, we are not addressing synchronization between two mechanical modes, firstly because they do not fulfill the synchronization requirement about the adjustment of their frequencies to the same oscillating frequency, but even because the oscillators need to be in a particular state of self-sustained oscillation. This simultaneously self-sustained oscillating condition is the one that we address, because, as in regular lasers [133], a mode competition phenomenon can avoid a situation with a multimode phonon laser scheme.

Concerning the last point, for a multimode mechanical system, it has been proved that the self-sustained oscillation, also addressed as phonon lasers, takes place just only for a single mode, the one whose threshold condition is satisfied first. This is the same as for conventional optical lasers, where a saturation phenomenon enables the lasing of one mode whilst suppress the gain for the others, as discussed in Fig. 4.6. Therefore, the motivation here is not to set two self-oscillating modes into the same synchronized frequency, but to be able to overcome this physical single mode limitation to get a multimode phonon laser. Besides that, what we observe is that once we overcome the limit, by means of an external modulated field set at a given frequency, the simultaneous phonon lasers get phase- or mode-locked. Here, by phase- or mode-locked what we mean is the resulting state of locking these two waves (even at different frequencies). When this mode-locking is obtained for a given detuning from each other, by phase-locking the beat of the two to a stable reference (both signals at the beat frequency), one can also say that the lasers are "phase-locked", even if they are at different frequency.

As a final remark, some previous works have demonstrated situations similar to two 
phonon lasing modes can coexist before the system entering into the synchronization regime, however, we may here differentiate between the different situations involving those works. In particular, in Ref. [139] both the optical modes and the mechanical modes in the total system lie into different physical resonators. That means that there is mode-competition between those mechanical modes because there are not even driven by the same optical field profile, thus these independent systems can reach the singlemode lasing state independently of each other. A similar situation is a case in Ref. [140], but with only one optical field profile. Here, however, the two mechanical modes are located at different physical membranes, thus resulting in a system where the mechanical modes are not even overlapping into the same structure (as it is in our case). Moreover, in the last work, both membranes can be driven by piezo-electric controllers, thus resulting in not enabling to freely evolve the system into their native mode competition state.

\subsubsection{Comparative stability analysis}

To characterize both the linewidth and the stability of the oscillators, we analyze their phase noise $L(f)$ in both the SSO and MMO states in Figs. 4.10. First and foremost, a measurement of the phase noise of a mechanical mode thermally driven and a SSO mode was performed in order to be able to distinguish both situations. These measurements are presented in Fig. 4.10(a) for the case where P1 is self-oscillating. Here, we can see an appreciable difference between these two cases as the thermally driven mode had a very low amplitude, thus resulting in a huge contribution of frequency noise sources related to the white noise. Once either $\mathrm{P} 1$ or $\mathrm{P} 2$ is in the self-oscillating regime, we observe that, in both cases, the different noise contributions are the ones that can be expected in $\mathrm{OM}$ oscillators: the white phase $\left(1 / f^{0}\right)$, white frequency (random phase walk, $\left.1 / f^{2}\right)$, and flicker frequency $\left(1 / f^{3}\right)$ noise types [101], in good agreement with the Leeson's model [112]. Phase noise values around $-100 \mathrm{dBc} / \mathrm{Hz}$ at $100 \mathrm{kHz}$ were observed, on par with other OM microwave oscillators $[44,101,111,114,117]$ as well as with our results in the previous chapter. From the fit of the random phase walk $\left(1 / f^{2}\right)$ at Fig. 4.10(a,b), a Lorentzian linewidth of $\Gamma_{\text {eff,P } 2} / 2 \pi \simeq 31 \mathrm{~Hz}$ is extracted for P2 in the SSO and $\Gamma_{\text {eff,P1 }} / 2 \pi \simeq 37 \mathrm{~Hz}$ and $\Gamma_{\text {eff,P1 }} / 2 \pi \simeq 36 \mathrm{~Hz}$ for $\mathrm{P} 1$ and $\mathrm{P} 2$ in the MMO, respectively.

(a)

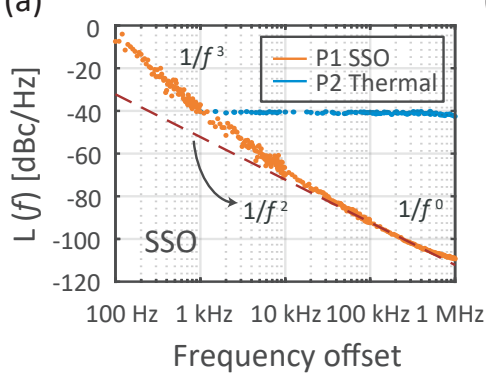

(b)

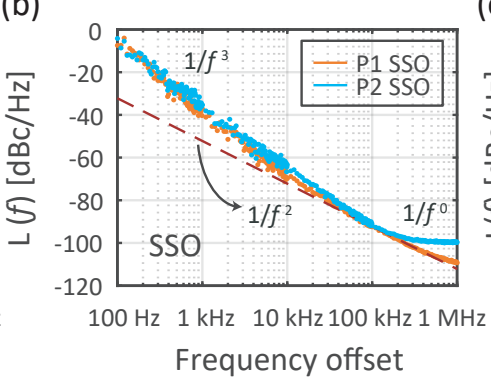

(c)

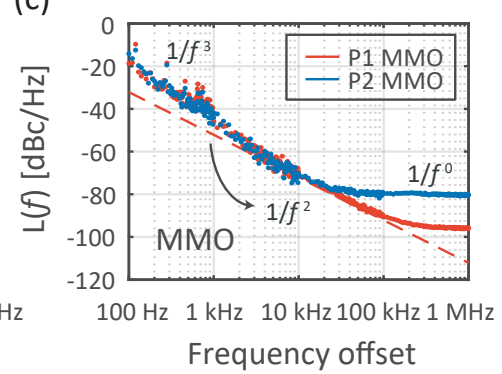

Figure 4.10: (a) Phase noise $L(f)$ from a thermally driven mechanical mode and a SSO mode. (b) Comparison of the phase noise between the two separately SSO states of P1 and P2. (c) Comparison of the phase noise between the two confined mechanical modes in the MMO regime.

The most interesting feature arises in the low frequency offset regime, where we 
(a)

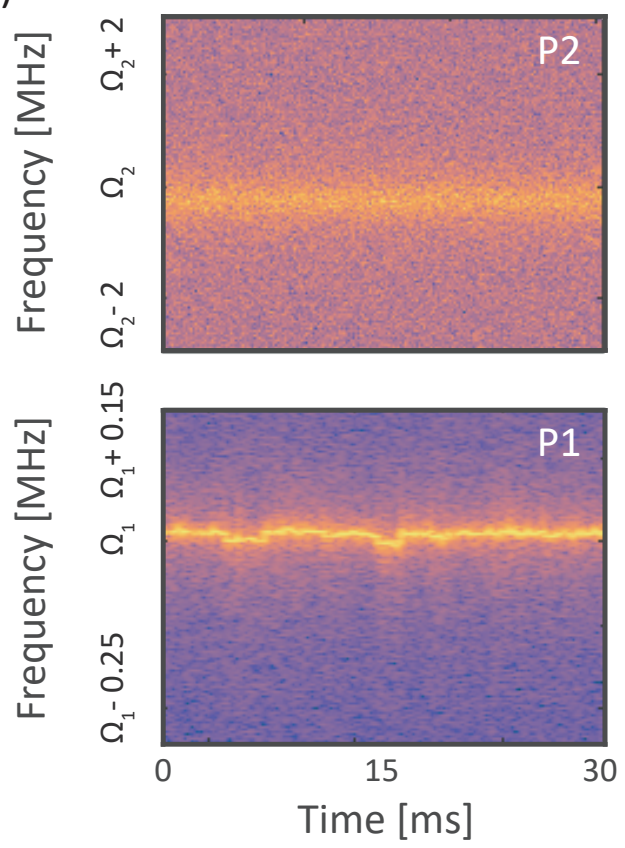

(b)

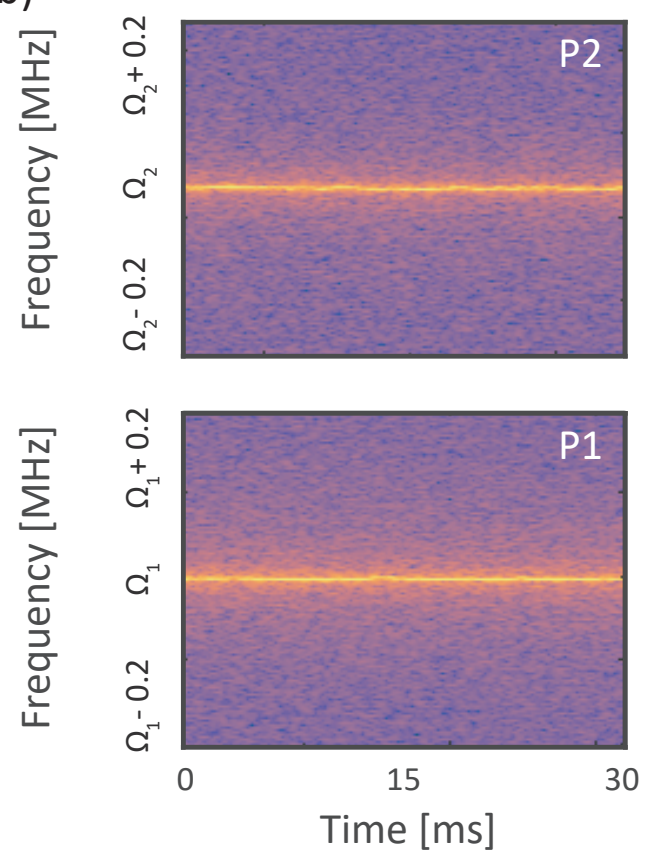

Figure 4.11: (a) Time evolution of the recorded spectra for the P2 (top) and P1 (bottom) modes for SSO lasing in mode P1. (b) Time evolution of the recorded spectra for the P2 (top) and P1 (bottom) modes for MMO lasing state.

can see a difference in the phase noise from SSO and MMO. For all frequencies below $\sim 10 \mathrm{kHz}$ (where the white noise background is insignificant), the phase noise improves when the two mechanical modes are in the multimode regime. For these frequencies, the phase noise has the largest contribution related to flicker frequency noise, judging from the slope of this curve. Moreover, as another measurement of the stability of the signal in a low averaging time range, we can extract the RMS jitter from the phase noise data through Eq. 2.14.

Table 4.1: Comparison of the RMS jitter of the SSO and MMO states.

\begin{tabular}{cccccc}
\hline Case & Reference & $f_{R F}(\mathrm{GHz})$ & $\begin{array}{c}J_{R M S}(\mathrm{ps}) \\
100 \mathrm{~Hz}-1 \mathrm{kHz}\end{array}$ & $\begin{array}{c}J_{R M S}(\mathrm{ps}) \\
100 \mathrm{~Hz}-1 \mathrm{MHz}\end{array}$ & $\Gamma_{\text {eff }} / 2 \pi(\mathrm{Hz})$ \\
\hline & & & 3410 & - & - \\
Isolated P1 SSO & {$[120]$} & 0.065 & 92.3 & 92.4 & 4 \\
P1 SSO & {$[101]$} & 3.870 & 218.7 & 219.1 & 38 \\
P2 SSO & {$[144]$} & 3.843 & 219.0 & 220.0 & 51 \\
P1 MMO & {$[144]$} & 3.783 & 74.4 & 74.8 & 36 \\
P2 MMO & {$[144]$} & 3.789 & 75.8 & 76.5 & 38 \\
& {$[117]$} & 0.112 & - & 10.1 & - \\
\hline
\end{tabular}

The obtained values of the RMS jitter are presented in Table 4.1, where Ref. [101] corresponds to the isolated OMO presented in Chapter 2 and Ref. [144] corresponds to the results addressed in this chapter. From the RMS jitter we can observe that once 


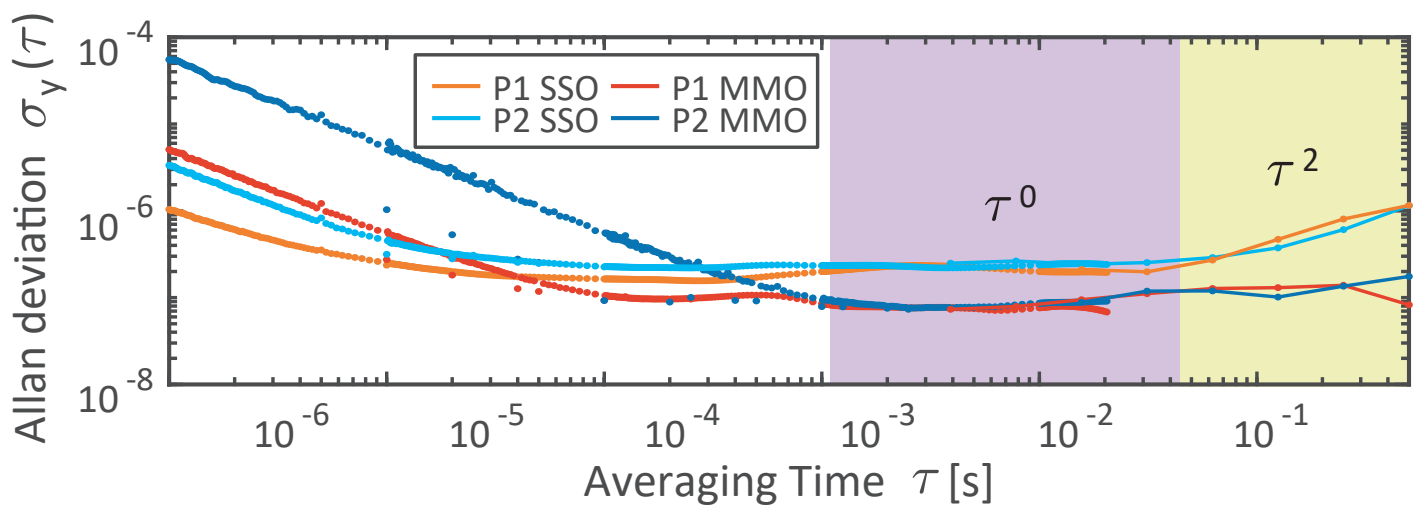

Figure 4.12: Allan deviation for P1 and P2 in the SSO and MMO states.

the system reaches the multimode oscillating regime, as it was discussed from the phase noise measurement, an improvement in the stability of the system as the RMS jitter is highly reduced for both oscillators. This can be observed by comparing the values of $\mathrm{P} 1$ SSO and P2 SSO versus P1 MMO and P2 MMO, where an improvement of $J_{R M S}$ from 220 ps to 75 ps is obtained.

The time evolution of the recorded spectra for single-mode and multimode lasing are presented in Fig. 4.11(a,b). A small jitter of the lasing frequency can be seen in panel (a), which we attribute to the effect of fluctuations (thermal, optical power, fiber taper) on multiple competing phenomena, in particular the optical spring effect. Interestingly, when the external modulation is active (panel (b)), we observe that the stability of both lasing modes is significantly enhanced.

The long-term stability is also quantified using the Allan deviation $\sigma(\tau)$, as plotted in Fig. 4.12, obtained by combining the phase noise measurements and the spectral time traces for longer acquisition times. Here, we see that whereas for small intervals $\tau$ the SSO and the MMO perform similarly, the stability of the MMO is improved beyond $\tau>10^{-2}$ in the mode-locked state. This cannot be ascribed to locking to the external modulation as this is far off-resonant. Instead, it could be related to the MMO regime being sustained at significantly reduced intracavity power, as suggested by the relative heights in 4.9, thus reducing the spring effect caused by optical fluctuations [146]. Moreover, it is known that synchronized oscillators can become less prone to fluctuations as their effective mass is increased. A related effect could be in play here. Further studies should be undertaken to reveal the various contributions and the application potential of the increased stability in the Floquet lasing regime.

Our investigation shows that non-linear dynamics of Floquet modes can be used to enable multi-mode phonon lasing in OM systems. The analytical argument based on higher-order cross-mode corrections of the self-energy for intensity modulation at the difference of mode frequencies is underpinned by numerical simulations as well as an experimental demonstration. We show how the multimode lasing state can be reached experimentally, and reveal that its long-term stability is improved. The multimode cavity used in our experiments paves the way towards the integration of multiple coexisting $\mathrm{GHz}$ mechanical modes. The concepts we establish can be used to study the emergent physics of many-mode self-oscillating OM systems, in a fully controllable fashion. More remarkably, multimode lasing could be used for frequency conversion purposes, as in 
the previous chapter, as well as to generate complex microwave signals beyond simple narrow tones.

Finally, it must be pointed out that the work involving the multimode optomechanical experimental demonstration was developed in collaboration with the University of Malta and AMOLF. The measurements were performed with the experimental setup illustrated in the next section (see Section 4.4.1). Here, two similar setups were installed (independently) at the AMOLF institute in the Netherlands and the Nanophotonics Technology Center (NTC) in Spain, where different compatible and reproducible sets of measurements were obtained. The experimental measurements at AMOLF were performed during a research stay at that institute in the Photonic Forces group led by Ewold Verhagen. On the other side, the University of Malta (K. Pelka and A. Xuereb) took part in the theoretical description of the multimode process through the Floquet dynamics induced by a temporally modulated laser drive. The respective results involving both the theory and the experiments can be found at Ref. [144].

\subsection{Experimental setup}

In this section the driving multimode phonon lasing configuration setup is presented.

\subsubsection{Multimode oscillation driving setup}

The multimode self-oscillation measurements presented in this chapter have been performed with the block diagram of the laboratory setup depicted in Fig. 4.13. Here, a continuous-wave optical signal passes through an intensity modulator fed by a signal generator with a tunable frequency of $\Omega_{R F}=\Omega_{1}-\Omega_{2}$. This part of the system, shadowed in yellow, can be considered a modification of the setup modulation part in Section 3.4.1. In practice, however, the fed signal is a single RF tone at the required frequency equal to the difference of the two involved mechanical modes.

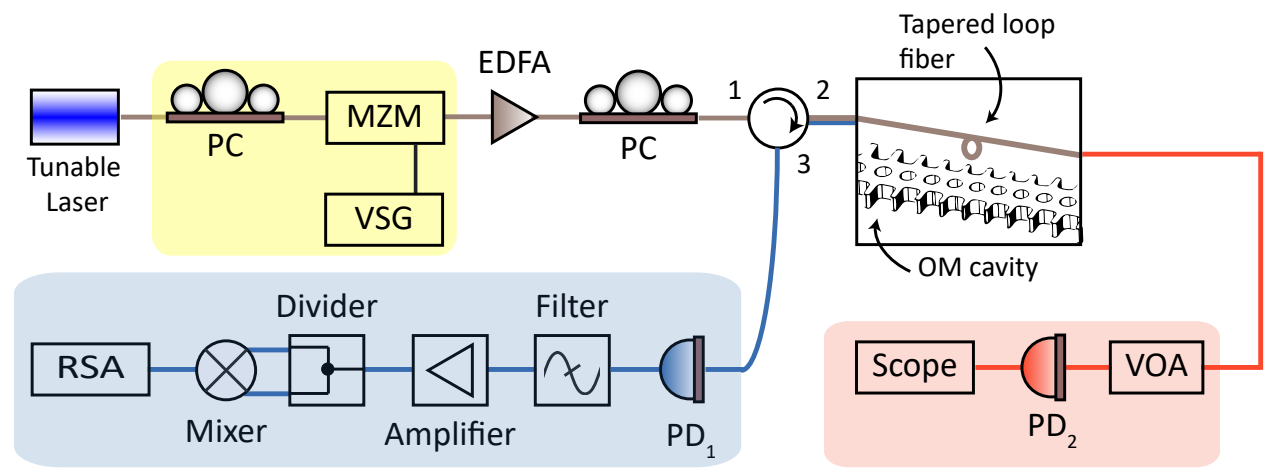

Figure 4.13: Block diagram of the experimental setup used in the driving and acquisition measurements of the multimode self-sustained oscillation regime for two mechanical modes.

The remaining part of the system can be considered similar to the ones presented in Chapters 2 and 3 excluding the reflection part. In this configuration, the reflection channel is used to measure the mechanical mode dynamics both in the single and the multimode regime. Because of that, different RF processing elements are used in order to ensure the proper acquisition of those signals. Here, the reflected signal from the 
light scattered by the cavity is first photodetected via a $12 \mathrm{GHz}$ bandwidth photodetector $\left(\mathrm{PD}_{1}\right)$ and then filtered with a bandpass filter (Mini-Circuits VBFZ-4000-S+). This filter is used to suppress the modulated frequency $\Omega_{R F}$ in the detected electrical signal which would overlap with the difference tone. Once the low-frequency range has been filtered, the signal is electrically amplified (Miteq MPN4-02001800-23P) and divided (Mini-Circuits 15542 ZFRSC-42) to feed the two input ports of an electrical mixer (MACOM M63C), where the difference and sum tones of the two lasing mechanical modes P1 and P2 are created. Finally, the resulting signal is analyzed with an RF spectrum analyzer. 


\title{
Chapter 5
}

\section{High-index disk optomechanics of supercavity modes}

\author{
Contents

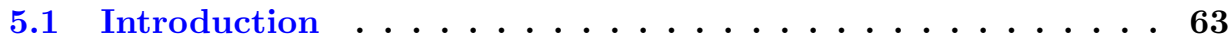 \\ 5.2 Quasi-BIC modes in high-index disks . . . . . . . . 64 \\ 5.2 .1 Scattering cross section . . . . . . . . . . . . . 64 \\ $5.2 .2 \quad$ Optomechanical coupling . . . . . . . . . . . . . . 65
}

5.3 Pedestal's substrate mechanical leakage . . . . . . . . 67

\subsection{Introduction}

Interaction between optical and mechanical waves in deformable cavities lies at the heart of cavity optomechanics [2], as we have seen throughout this thesis. Amongst the different approaches to build OMCs, their realization on high refractive index films using standard lithographic processes lead to the structure studied until now by introducing an engineered periodicity. Still, some limitations arise. For instance, the maximum mechanical frequency that can be attained is around $5 \mathrm{GHz}$, so processing microwave signals at higher frequencies requires for the use of higher-order harmonics that have less power. In addition, the complexity of the structure makes difficult the repeatability in the sense of having the same optical and mechanical frequency when multiple samples are fabricated.

In parallel to the recent advances in cavity optomechanics, the field of high-index nanophotonics [147] has come up with new strategies to build wavelength-size optical cavities in high-index films without using periodic mirrors. In particular, by properly tailoring the aspect ratio of a high-index disk, interference between different optical modes cancels out the far-field scattering and enables tight localization of the optical field inside the structure. Moreover, the so-called quasi-bound states in the continuum (quasi$\mathrm{BIC}$ modes) can arise, resulting in relatively high values of the $\mathrm{Q}$ factor $(\mathrm{Q}>100)[57,58]$. Notably, the strong field localization in single disks extremely enhances nonlinear effects such as second [58] or third harmonic generation [148]. However, another nonlinear effects such as OM interaction has not been studied so far in the aforesaid structures. 
Consequently, in this chapter, we theoretically explore the dispersive OM coupling in isolated high-index disks supporting quasi-BIC modes at telecom wavelengths. We show that, besides optical resonances, the disks also support mechanical resonances at microwave frequencies and they can be coupled to the optical resonance with significantly large values of $g_{0}$. Regarding the practical implementation, it is shown that having the disk supported by a thin silica pedestal, which is technologically feasible, would strongly suppress phonon leakage in the substrate ensuring material-limited mechanical losses, as in released $\mathrm{OM}$ cavities operating at room temperature. Our results suggest that these cavities could be interesting in applications where unresolved sideband operation is not mandatory. In addition, these cavity disks may also be used to build massive arrays of $\mathrm{OM}$ cavities to create $\mathrm{GHz}$-tunable $\mathrm{OM}$ metasurfaces for multiple applications.

\subsection{Quasi-BIC modes in high-index disks}

Optical confinement in subwavelengths volumes is critical for many photonic applications. Multiple approaches attaining this optical localization can be found in the literature as devices based on photonic crystals [93], plasmonic structures [149], ring resonators [150] or high-index dielectric nanoparticles [57], between others. Concerning the last ones, high-index dielectric nanoparticles can exhibit strong near-field confinement and tailorable field distributions in the subwavelength regime [151] due to the simultaneous support of both electric and magnetic Mie resonances [147]. However, a limiting factor of these dielectric systems can be found in the low optical quality factor of their resonances [58]. Therefore, to obtain high optical quality factors, commonly desirable for practical applications, the approach based on bound states in the continuum (BIC) is implemented.

Bound states in the continuum can be understood as waves that remain perfectly confined without any radiation even when they coexist with a continuous spectrum of waves that radiate energy away [152]. Despite mathematically these BIC modes should have infinitely large quality factors, a practical implementation may constrain this value. The finite sample size once they are physically implemented besides the absorption losses, the role of the substrate or fabrication imperfections as roughness, can lead to leaky resonances resulting in a lowered $\mathrm{Q}$ factor [153]. However, large Q factors can still be obtained by means of this concept, thus resulting in the so-called supercavity or quasi-BIC modes [57].

\subsubsection{Scattering cross section}

As previously introduced, the supercavity or quasi-BIC modes arise in wavelength-sized high-index dielectric disks as a result of the interference between different modes that allow reducing the radiation (or scattered) field. However, these disks must have a specific aspect ratio $h / d$, where $h$ the disk height and $d$ the disk diameter. In Ref. [57] it is predicted that a silicon disk with aspect radio $h / d=0.7308$, can reach $\mathrm{Q} \approx 200$ at telecom wavelengths due to the interference between Mie-type and Fabry-Perot-type modes. The electric field patterns at $\lambda=1559.1 \mathrm{~nm}$ and $\lambda=1555.8 \mathrm{~nm}$ of the quasi-BIC mode are shown in Fig. 5.1 for the Si and AlGaAs disks, respectively. Additionally, the computed far-field scattering profiles for each of the high-index disks are also presented. Through a Fano-resonance fit of the scattering cross-section profiles, the obtained optical quality factor for those modes resulted in a value of $\mathrm{Q} \simeq 237$ for the $\mathrm{Si}$ disk and $\mathrm{Q} \simeq$ 
268 for the AlGaAs disk. The Fano resonance was fitted according to [154]:

$$
F(\alpha)=A \frac{(\alpha+q)^{2}}{1+\alpha^{2}}
$$

where $\alpha=2 \frac{\lambda-\lambda_{0}}{\Gamma}$. Specifically, $\mathrm{A}$ is a constant factor, $\lambda_{0}$ and $\Gamma$ are the resonant wavelength and its linewidth, and $q$ accounts for an asymmetric parameter which can be interpreted as the amplitude of the resonant contribution relative to the background [155].
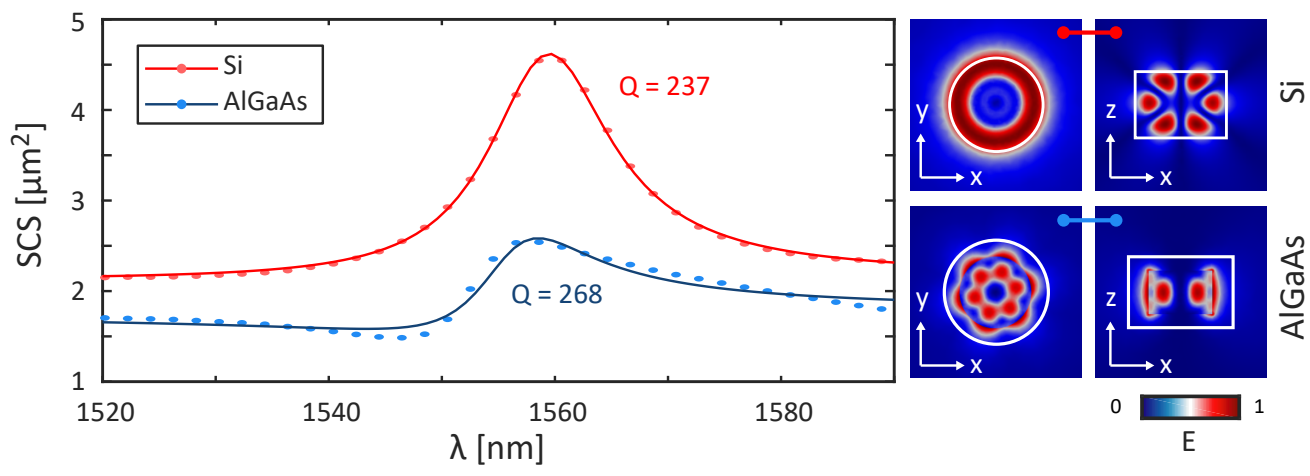

Figure 5.1: Simulated far-field scattering cross-section and Fano fits for the calculation of the estimated optical quality factor for a Si disk with $\mathrm{h}_{d}=589 \mathrm{~nm}$ and $\mathrm{r}_{d}=403 \mathrm{~nm}$ at $\lambda=1559.1 \mathrm{~nm}$ in red and an AlGaAs disk with $\mathrm{h}_{d}=635 \mathrm{~nm}$ and $\mathrm{r}_{d}=451.5 \mathrm{~nm}$ at $\lambda=$ $1555.8 \mathrm{~nm}$ in blue. Data points correspond to the simulated far-field scattering and the solid lines to the resulting fitted Fano resonance. (Insets) Supercavity or quasi-BIC mode profiles of the total electric field $|\mathbf{E}|^{2}$ for the Si disk at the top panels and the AlGaAs disk at the bottom panels.

\subsubsection{Optomechanical coupling}

We also computed the mechanical resonances of both disks up to $30 \mathrm{GHz}$ using COMSOL Multiphysics. Once the displacement field $\mathbf{u}$ was obtained, the OM coupling rate $g_{0}$ was calculated for each mechanical mode from both the moving boundary (MB) and the photoelastic (PE) effect contributions, as described in Sec. 2.2.2. The obtained calculations are presented in Fig. 5.2 for a $\mathrm{Si}$ and an AlGaAs disk keeping the aspect ratio required to have the supercavity mode. The simulations were performed assuming that the cavities can be well approximated as a closed system because of the relatively high optical Q. In other words, we neglected dissipative coupling and only accounted for dispersive coupling using the analytical expressions [30]. Both the Si and AlGaAs materials were simulated as anisotropic media whose properties are summarized in Table $5.1[156,157]$. The used Si photoelastic constants were the one already employed in the previous chapters, $p_{11}=-0.09, p_{12}=0.017$ and $p_{44}=-0.051$. However, the AlGaAs parameters were assumed to be equal to those of GaAs as they have been unknown in the literature, thus resulting in $p_{11}=-0.165, p_{12}=-0.140$ and $p_{44}=-0.072$ [156].

Figure 5.2(a) shows the obtained values of $g_{0} / 2 \pi$ for the Si disk and Fig. 5.2(b) those for a AlGaAs disk. As insets labeled figures the total mechanical displacement 
Table 5.1: Optical and mechanical properties of the high-index disks employed materials.

\begin{tabular}{cccccccccc}
\hline & Ref. & $\varepsilon$ & $\begin{array}{c}\rho \\
{\left[\mathrm{kg} / \mathrm{m}^{2}\right]}\end{array}$ & $\begin{array}{c}c_{11} \\
{[\mathrm{GPa}]}\end{array}$ & $\begin{array}{c}c_{12} \\
{[\mathrm{GPa}]}\end{array}$ & $\begin{array}{c}c_{44} \\
{[\mathrm{GPa}]}\end{array}$ & $p_{11}$ & $p_{12}$ & $p_{44}$ \\
\hline $\mathrm{Si}$ & {$[157]$} & 13 & 2329 & 166 & 64 & 80 & -0.09 & 0.017 & -0.051 \\
$\mathrm{AlGaAs}$ & {$[156]$} & 11.42 & 4540 & 119.5 & 55.4 & 59.1 & -0.165 & -0.140 & -0.072 \\
\hline
\end{tabular}

pattern of the mechanical modes with higher coupling rates than $200 \mathrm{kHz}$ are presented. Remarkably, $g_{0} / 2 \pi \approx 800 \mathrm{KHz}$, similar to what is obtained in OMCs implemented on 1D periodic beams, is obtained for different mechanical modes. It can be noted that in both cases the highest $\mathrm{OM}$ coupling rates with values of $-737 \mathrm{kHz}$ and $-754 \mathrm{kHz}$ for $\mathrm{Si}$ and AlGaAs, respectively, are obtained for the same mechanical mode profile depicted in S2 and A2. The mechanical frequency of this mode varies from one disk to the other, but higher frequency modes can be found in the Si disk. Remarkably, a mechanical mode at $29.97 \mathrm{GHz}$ with $g_{0} / 2 \pi=279 \mathrm{kHz}$ can even be found in the Si disk (mode S5). The presence of such high-frequency mechanical modes with high coupling rates could enable the operation of those systems in applications for biology or chemistry in liquid environments [158]. Additionally, it could be used to extend the frequency regime of the devices demonstrated in previous chapters up to the millimeter-wave regime.
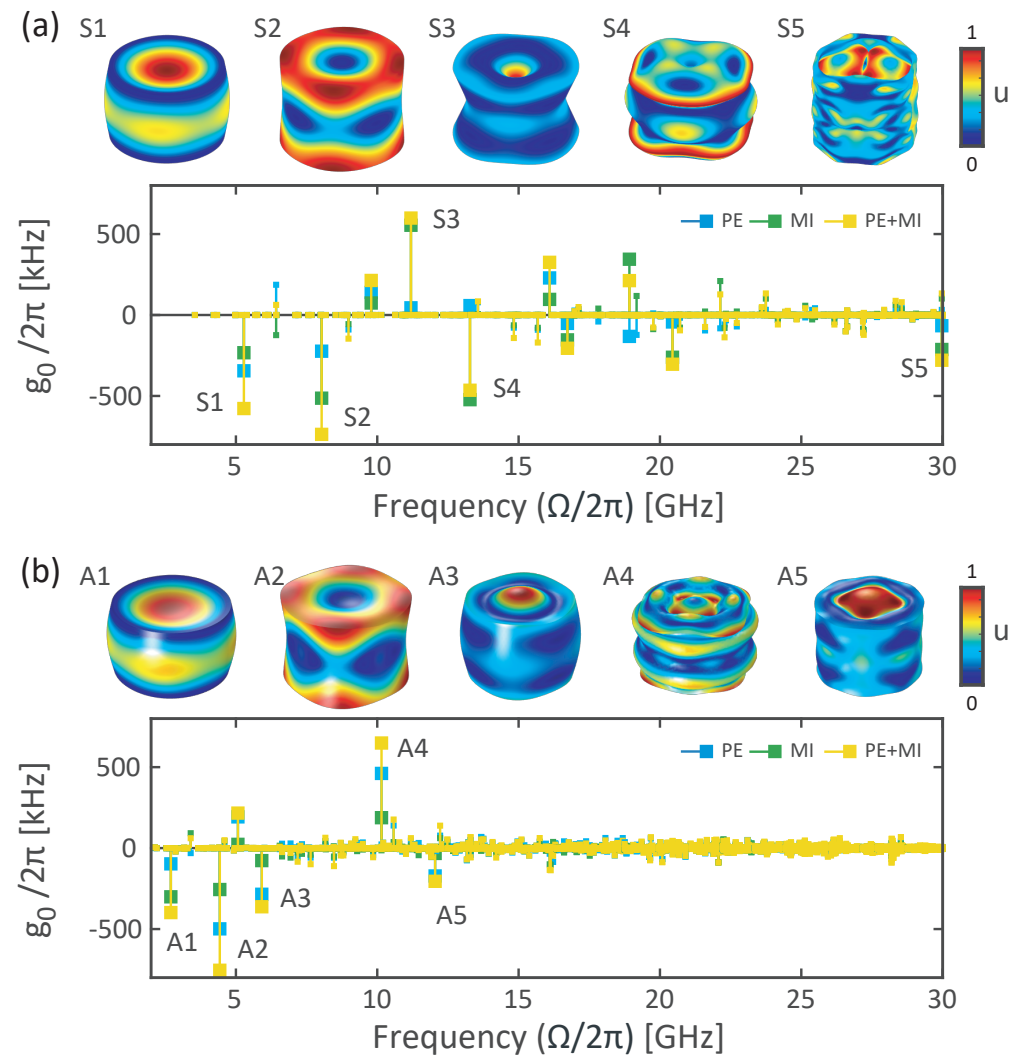

Figure 5.2: (a) OM coupling rate $\left(g_{0} / 2 \pi\right)$ and mechanical mode profiles for a Si disk with $\mathrm{h}_{d}=589 \mathrm{~nm}$ and $\mathrm{r}_{d}=403 \mathrm{~nm}$. (b) AlGaAs disk OM coupling rate with $\mathrm{h}_{d}=635 \mathrm{~nm}$ and $\mathrm{r}_{d}=451.5 \mathrm{~nm}$. 


\subsection{Pedestal's substrate mechanical leakage}

Up to now, we have seen that high confined mechanical modes in the studied highindex disks exhibit high $g_{0} / 2 \pi$ values once they could with the quasi-BIC modes, thus enabling OM transduction. Nevertheless, these systems were studied theoretically for a suspended disk, meaning that the system was completely surrounded by air (or vacuum, since for $\mathrm{GHz}$ frequencies mechanical leakage in air can be considered to be negligible). This approach ensured that the mechanical vibrations were completely bounded to the disk and the phonon lifetime will be uniquely limited by the absorption losses in the material.

(a)

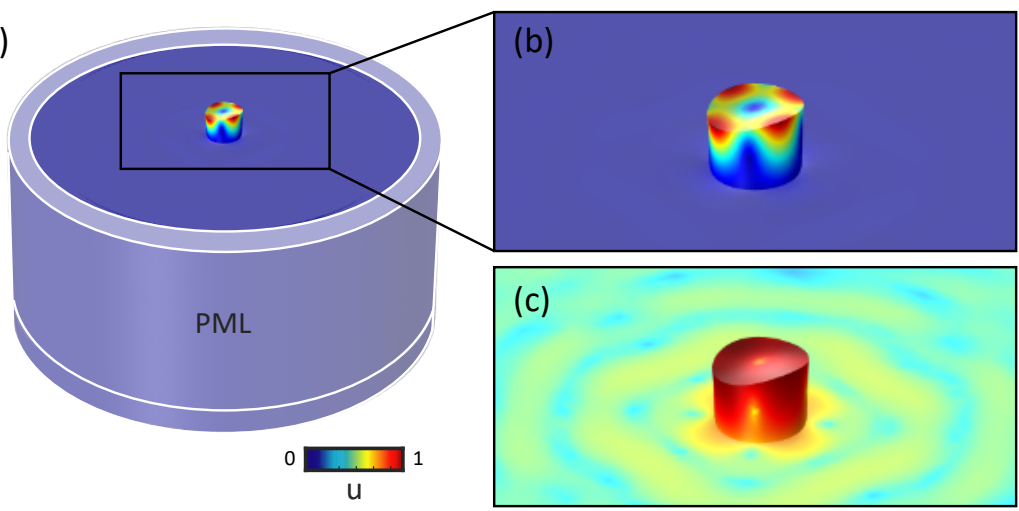

Figure 5.3: (a) Mechanical integration domain of a high-index silicon disk placed on a silica substrate. Mechanical PML conditions are set at the boundaries of the structure as well as at the bottom of the substrate. (b) Close view of a mechanical confined mode in a linear scale for the original A2 mode in Fig. 5.2. (c) Close view of the last mechanical mode in logarithmic scale where the phonon leakage into the substrate can be appreciated.

In terms of practical implementation, however, this approach is not realistic since in a real situation the high-index disks must be placed on a substrate, typically silicon dioxide $\left(\mathrm{SiO}_{2}\right)$. Since $\mathrm{SiO}_{2}$ is a low-index material, it can be expected that the presence of the substrate will have a low influence on the optical properties. Moreover, the optical Q factor can be even improved by further engineering of the surroundings of the disks [57]. But, in the case of the mechanical properties, the sound velocity in $\mathrm{SiO}_{2}$ is smaller than in either $\mathrm{Si}$ or AlGaAs, which means that phonons will tend to leak towards the $\mathrm{SiO}_{2}$ substrate, resulting in prohibitive mechanical losses. An example of the phonon leakage into the substrate can be appreciated in Fig. 5.3, where a modified version of the S2 mode from the last section is studied. In Fig. 5.3(a) the overall mechanical structure is presented, where a Si disk (the analysis is equivalent for the AlGaAs disk) is on top of a $\mathrm{SiO}_{2}$ substrate. It must be noted that mechanical Perfectly Matched Layers (PML) were imposed in the bottom and the surroundings of the substrate depicted with a shadowed volume, and all the other material interfaces were kept as free boundaries. Despite it may seem that we can localize mechanical modes, as can be observed in the close view of Fig. 5.3(b), phonon leakage into the substrate is present. Indeed, Fig. 5.3(c) shows the same mechanical mode profile on a logarithmic scale, where it can be appreciated that the displacement leakage can be now easily identified. A quantitative value of this 
phononic confinement can be obtained through the calculation of the localization factor $(L F)$, which can be estimated as:

$$
L F=\frac{\int_{D i s k}\left|\mathbf{u}(\mathbf{r}, t)^{2}\right| d \mathbf{u}}{\int_{A l l}\left|\mathbf{u}(\mathbf{r}, t)^{2}\right| d \mathbf{u}}
$$

which is defined as the ratio between the total displacement localized in the disk over the total displacement in all the mechanical structures (both the disk and the surroundings are taken into account). That means that a total confined mode (no substrate) should give a localization factor of 1 . Nonetheless, for the given mechanical mode, the $L F$ was estimated to be 0.02 , even despite the maximum displacement is located at the top of the structure. It must be noted that, according to the phononic displacement profiles providing the highest $\mathrm{OM}$ coupling rates obtained in the last section, almost none of them could be feasible with the direct substrate approach. This is because once the disk stays on top of the substrate most of these modes can not freely move as in the suspended case, thus limiting the existing mechanical modes to modes localized in the top of the disk.

(a)

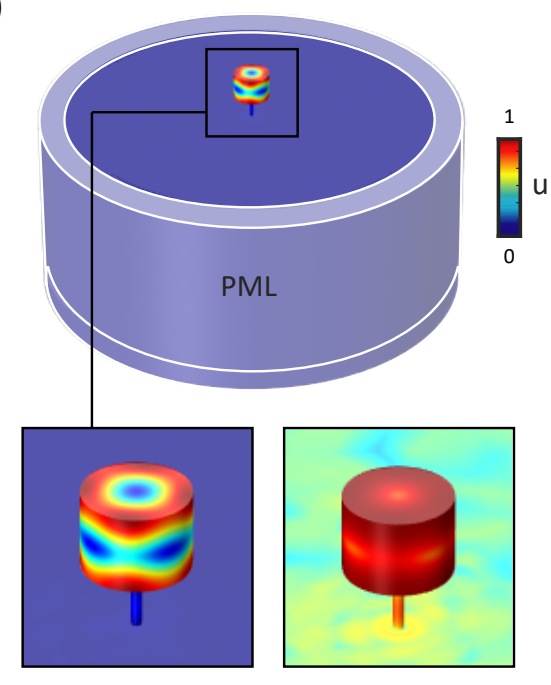

(b)
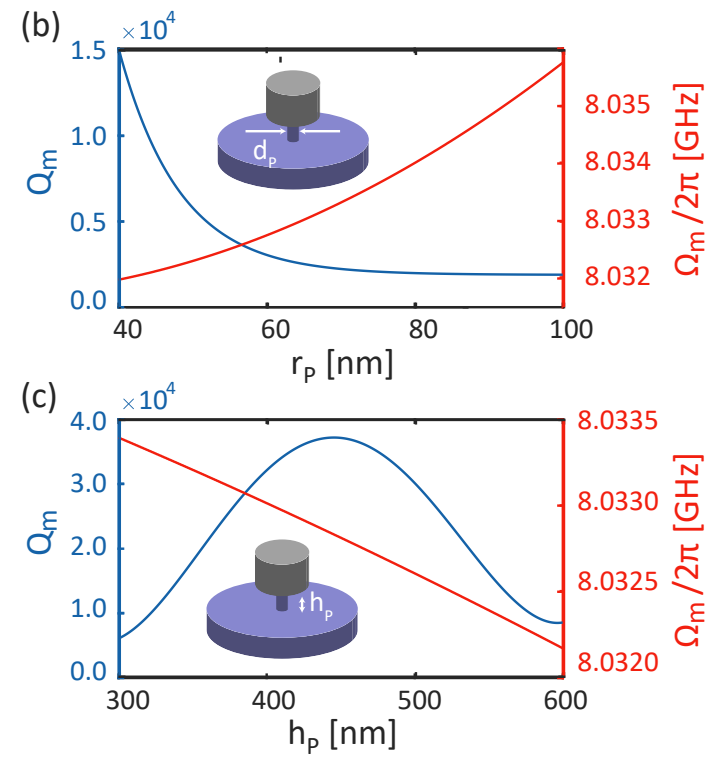

Figure 5.4: (a) Mechanical mode simulation domain with insets views depicting the confined A2 mode when the high-index disk is placed on top of a silica pedestal connected to the substrate. (b) Evolution of the mechanical quality factor and the oscillation frequency as a function of the pedestal radius. (c) Evolution of $Q_{m}$ and $f_{m}=\Omega_{m} / 2 \pi$ as a function of the pedestal height.

Because of the observed phonon leakage, this is the reason why released structures are conventionally used in integrated OMCs. Phonon leakage can be highly reduced if we assume the disk to be placed on a thin pedestal, following the approach presented in Ref. [158] for GaAs disks and in Ref. [159] for high-index waveguides. Thus, we assumed that the high-index disks were staying on a thin $\mathrm{SiO}_{2}$ cylindrical pedestal (height $h_{p}$ and diameter $d_{p}$ as depicted in the insets of in Fig. 5.4). Under this proposed configuration, we studied the evolution of the mechanical quality factor $\mathrm{Q}_{m}$ as a function of the pedestal height and diameter for the S2 mode in the Si disk, the one providing the highest OM 
coupling rate. A picture of the overall system can be seen in Fig. 5.4(a). Here, the insets figures show the total displacement profile both in linear and logarithmic scale for the sake of completeness. In contrast to the case where the disk was directly on top of the substrate, here we can see that with the pedestal approach we can still obtain the original phononic field profile at the same time that the confinement is improved. Through the calculation again of the localization factor, a value of $L F=0.97$ (for the case of $\mathrm{h}_{p}=500 \mathrm{~nm}$ and $\mathrm{r}_{p}=50 \mathrm{~nm}$ as an example) is obtained, which means that the mechanical mode is almost totally confined in the disk even when it is placed at the top of the pedestal.

Regarding the calculation of the mechanical quality factor, we neglected material absorption losses being our objective to estimate the structural mechanical Q factor. It has to be noted that in this configuration only the mechanical modes with a negligible displacement in the middle of the disk will still be localized. Figure5.4(b,c) summarizes the evolution of the $\mathrm{Q}_{m}$ as well as the shift in frequency of the mechanical mode as a function of the height and radius of the $\mathrm{SiO}_{2}$ pedestal.

As shown in Fig. 5.4(b) an increase of pedestal radius results in a reduction of the mechanical Q factor, as expected. This is due to the fact that the S2 mode profile will be disturbed if the pedestal diameter extends beyond the zero displacement region localized at the middle of the disk. In that case, the mechanical mode could leak into the substrate through the pedestal thus reducing the mechanical quality factor. If we assume that $\mathrm{Q}_{m} \simeq 10^{3}$ at room temperature due to material absorption [31], this will be the dominant mechanism for phonon losses for very thin radii. In other words, assuming $\mathrm{r}_{p}<80 \mathrm{~nm}$ and $\mathrm{h}_{p} \simeq 450 \mathrm{~nm}$ the mechanical properties will be the same as in the case of released OMC cavities when operating at room temperature. In Fig. 5.4(c) we can see that there is an optimum value of $\mathrm{h}_{p}$ that maximizes $\mathrm{Q}_{m}$. We believe that this is due to a resonant effect in the pedestal that contributes to maximize the mechanical field in the disk when $\mathrm{h}_{p}=450 \mathrm{~nm}$.

The previous results suggest that supercavity modes in high-index disks may play an important role in cavity optomechanics. Even though operation in the sideband-resolved regime seems unattainable, the large values of $g_{0}$ would ensure transduction of the GHzscale mechanical modes as well as its manipulation either by cooling or heating them. In particular, blue-detuned laser driving at high power could ensure the formation of broad (> $100 \mathrm{GHz}$ ) optical frequency combs [101] in a wavelength-sized structure, opening the door towards ultra-compact microwave photonics. Notice also that the dimensions of the disks under study are smaller than those reported in [156,158], which support high-Q optical and mechanical whispering gallery modes. This further miniaturization to sizes of the order of the wavelength allows for accessing mechanical modes at higher frequencies, going even beyond $10 \mathrm{GHz}$, which is highly challenging in OMC cavities [160].

It may be noted that in our study, we have only considered dispersive coupling even though the system presents non-negligible radiation losses. Still, we have observed small variations $(<1 \mathrm{kHz})$ of the calculated $g_{0}$ when the lateral size of the simulation volume is changed by a factor of 10 times higher. This means that our results should be considered as a good approximation to the dispersive behavior of the system. Further calculations using quasi-normal modes [161] would enable us to calculate the dissipative coupling, which could shed more light on the OM properties of these kinds of systems.

In terms of practical applications, in comparison with OMC cavities, high-index disks can be excited from the far-field by using special arrangements of polarization [58], which eases the driving conditions. This way, we may think of OM metasurfaces formed by ar- 
rays of such disk so that their properties could be dynamically tuned at sub-nanosecond speeds, leading to ultrafast reconfigurable metasurfaces. The high mechanical frequencies would also ensure operation in liquid environments relevant in biology and chemistry [158]. This could enable the detection of organisms smaller than bacteria, such as viruses or proteins, in on-chip optomechanical arrays [20]. The main results in this chapter were published and can be found at Ref. [162].

Concerning the issue of the phonon leakage in the substrate, in the next Chapter we introduce a novel architecture that allows to get confined mechanical modes as well as large OM interaction without either completely releasing the substrate or introducing a thin pedestal. 


\title{
Chapter 6
}

\section{Strong light-sound interaction in unreleased Si-based waveguides}

\author{
Contents

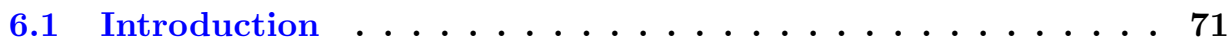 \\ 6.2 Description of the light - sound waveguiding system $\ldots \ldots 73$ \\ 6.3 AO interaction and Brillouin gain $\ldots \ldots \ldots \ldots \ldots$ \\ 6.4 Intramodal first order mode interaction $\ldots \ldots \ldots \ldots 77$ \\ 6.4.1 Forward Stimulated Brillouin Scattering . . . . . . . . . . . 77 \\ 6.4.2 Backward Stimulated Brillouin Scattering . . . . . . . . 80
}

6.5 Enhancement of the Brillouin gain at low temperature . . . 82

6.6 Practical implementation . . . . . . . . . . . 82

\subsection{Introduction}

In the nano-optomechanical systems studied until now, either in crystal cavities (Chapters 2 to 4 ) or in high index disks (Chapter 5), it has been demonstrated that a strong interaction between the optical confined modes and the mechanical vibrations in the structure can be found $[101,144,162]$. However, in order to prevent mechanical leakage into the substrate, most of the strong AO interactions found in the literature $[2,30,31,59,60]$ made use of released ultra-thin silicon membranes ( $\sim 200 \mathrm{~nm}$ thickness). That means that the layer under the structure has to be removed, thus resulting in some practical drawbacks. Part of these handicaps are related to the fabrication procedure because removing the substrate turns the structures highly fragile, and the releasing process itself can degrade other components in the chip due to the use of HF baths. The last point can even avoid the co-integration of multiple photonic, mechanical and electronic elements. However, some limitations can also be found in their functionality since the membrane release limits the device capability for dissipating heat [163], but also reduces the mechanical robustness and hinders the realization of large-area OM devices on a single chip.

As a consequence, the study of $\mathrm{AO}$ systems where the mechanical leakage could be prevented without releasing the structure results highly significant. In these terms, in this chapter, we explore a vertical engineered photonic-phononic structure showing that 
a thick silicon nitride layer between the silicon guiding core and the silica substrate contributes to reduce $\mathrm{GHz}$-frequencies phonon leakage enabling a large $\mathrm{AO}$ interaction. In practice, it is demonstrated via the interaction between two optical waves through a Stimulated Brillouin Scattering (SBS) process. It must be noted that despite the SBS and the cavity optomechanics fields have been considered for a long time mostly disconnected, it is possible to connect both theories [62,82]. Moreover, close connections between the most characterizing parameters in these branches, namely the Brillouin gain $\left(G_{B}\right)$ and the OM coupling rate $g_{0}$, can be obtained. Therefore, despite the results presented in this chapter are attained for a SBS process, it could be also possible to extrapolate them to the cavity optomechanics field. Indeed, it would be possible to implement $\mathrm{OM}$ cavities using the vertical confinement structure that is developed in this chapter.

As noted in Chapter 1, the SBS phenomenon is a third-order nonlinear process arising from photon-phonon interaction in material systems supporting both optical and acoustic propagating waves $[70,71]$, by which optical photons are coupled to acoustic phonons $[69,80]$. With this picture in mind, for many years, optical fibers were the most extended platform to observe SBS and related phenomena since they allow large interaction distances $(\gg \mathrm{km})$ with negligible losses [164]. Indeed, many applications - mainly in the context of microwave photonics [165]- were developed, spanning from tunable microwave filters [166] to optical pulse storage [167]. The current trend towards miniaturization and on-chip integration of optical devices has also been followed in SBSrelated phenomena and applications [61]. The first demonstration of on-chip SBS made use of a chalcogenide glass $\left(\mathrm{Al}_{2} \mathrm{~S}_{3}\right)$ as guiding core material for both light and sound [75]. As in optical fibers, $\mathrm{AO}$ interaction via electrostriction and photoelasticity in the waveguide core was the main responsible for SBS [98]. Despite that this approach enables large Brillouin gain and multiple SBS-based on-chip functionalities [168,169], it is not compatible with CMOS technology, which prevents massive integration with mainstream silicon photonic and electronic components.

On the other side, silicon was originally disregarded as an appropriate material for integrated SBS since when placed over silica - as in standard silicon-on-insulator wafers - it cannot confine mechanical waves, which prevents strong AO coupling [82]. SBS in silicon waveguides (as well as OM interaction in cavities, as shown in the previous chapters) requires the waveguide (cavity) to be at least partly released so that phonon pathways towards the silica substrate are strongly inhibited $[79,159,170]$. Noticeably, the strong field confinement in high-index wavelength-size silicon core does not only result in enhanced $\mathrm{AO}$ volumetric interaction due to the large photoelastic effect in silicon; in addition, the photon-phonon interaction is mediated by the radiation pressure exerted by the optical field on the interfaces, which scales up with the electric permittivity contrast at the boundary [30,98]. This has led to experiments reporting values of the Brillouin gain $\left(G_{B}\right)$ well above $10^{3} 1 / \mathrm{Wm}[159,170]$, which confirms the appropriateness of silicon as a material for integrated SBS. However, besides some recent exciting demonstrations, such as the case of a silicon Brillouin laser [171], the need for partial release of the silicon core complicates the fabrication (as it requires HF baths), reduces thermal dissipation, prevents the introduction of more sophisticated AO elements (required for building chip-scale data processors based on SBS [61]) and reduces the mechanical robustness of the device, as previously introduced. Therefore, a silicon-compatible platform displaying mechanical robustness, large Brillouin gain and low propagation losses whilst simultaneously not requiring the release of the silicon core is still missing [61]. 
Recently, a novel approach for unreleased-silicon SBS based on a geometric softening technique was proposed [172]. Here, two high aspect ratio silicon waveguides placed on a silica substrate and spaced by a thin slot were shown to provide large $G_{B}$ values for both backward and forward configurations. However, fabrication of this slotted structure becomes complicated because the thin slot and optical propagation losses may become quite large because of the large area of the sidewalls boundaries, so SBS has not been demonstrated in this system to the best of our knowledge. Silicon nitride waveguides have also been shown as good candidates for unreleased SBS-based devices mainly because they show extremely low linear losses and negligible two-photon absorption [173]. In this case, phonons are not confined in the waveguide core, which results in $G_{B}$ values that are orders of magnitude smaller than in released silicon waveguides [174] and, consequently, high operating optical power and ultralong waveguides are required.

Taking this into account, we propose and analyze numerically an unreleased-silicon based platform obtained by vertical engineering a $\mathrm{Si}$ waveguide over a $\mathrm{Si}_{3} \mathrm{~N}_{4}$ substrate showing that a reduction of the phonon leakage besides a strong localization of the mechanical mode in the silicon core can be feasible, as discussed in the next sections. We have to emphasize that even though only SBS is analyzed in this new system (or guided AO interaction) the feasibility to localized mechaical waves could also be used to build OM cavities similar to the ones presented in previous chapters but without the need for releasing the silicon core.

\subsection{Description of the light - sound waveguiding system}

The proposed unreleased platform must be able to simultaneously confine both the optical field profiles and the transduced mechanical wave into the same spatial extension. Hence, the key idea is to place between the silicon high-index guiding core and the silica low-index substrate an intermediate layer made of a material having both a refractive index lower than silicon (to ensure photon confinement in the silicon layer) and a sound speed higher than silicon (to avoid phonon leakage from silicon to silica). Fulfilling this requirement and with the aim of turn our system completely CMOS compatible, silicon nitride $\left(\mathrm{Si}_{3} \mathrm{~N}_{4}\right)$ was chosen as the intermediate material layer. Noticeably, even though it depends on its state (crystalline or amorphous) as well as the deposition method, both the speed of sound and stiffness are higher in $\mathrm{Si}_{3} \mathrm{~N}_{4}$ than in silicon [174-176], which is a basic requirement to reduce mechanical leakage from the silicon core to the silica substrate.

The final system results in the scheme proposed in Fig. 6.1(a), where a transverse cross section of the proposed waveguiding system is shown. It consists of a Si core (width $w$, thickness $b$ ) that lies upon a $\mathrm{Si}_{3} \mathrm{~N}_{4}$ layer (width $w$, total thickness $t$, etched thickness $e$ ) that is placed over a silica substrate, which we assume to be semi-infinite. Regarding the used boundary conditions for the SBS simulations that will be discussed from now on, optical PMLs were set at all the external surroundings of the structure while mechanical PMLs were only applied at the bottom and lateral sides of the system. All the other boundaries in the mechanical simulations were considered to be free boundaries to let the structure freely evolve as in the real implementation.

Concerning the aforesaid simulations, the optical properties of the system were described with the refractive index of the material at telecom wavelengths considered to be $n_{\mathrm{Si}_{i}}=3.48, n_{\mathrm{SiO}_{2}}=1.44$ and $n_{\mathrm{Si}_{3} \mathrm{~N}_{4}}=2.02$. Beyond that, the mechanical properties were set as a function of the material model employed to simulate the material. In partic- 
(a)

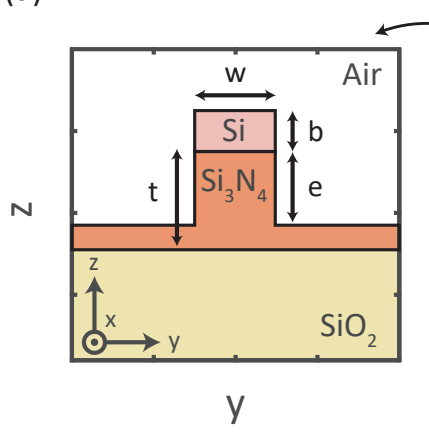

(b)

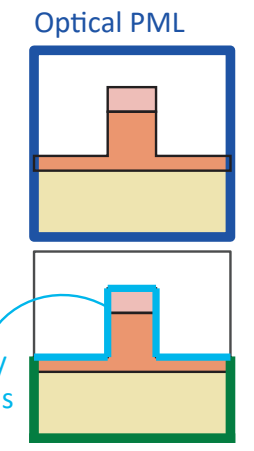

Mechanical PML

Figure 6.1: (a) Material cross-section of the $\mathrm{Si}_{-} \mathrm{Si}_{3} \mathrm{~N}_{4}$ photonic-phononic waveguide system with their labeled dimensions. (b) Optical and mechanical boundary conditions set at the SBS simulations.

ular, the silicon and silicon nitride layers were described as anisotropic materials and the silica substrate was considered to be isotropic. For the anisotropic materials, the main parameters defining the system were the elasticity matrix and the material density. For silicon we employed the already mentioned parameters presented in Chapter 2. Meanwhile, the silicon nitride stiffness constants in Voigt notation were $\left[c_{11}=4.33 \cdot 10^{11}\right.$, $\left.c_{33}=5.74 \cdot 10^{11}, c_{44}=5.74 \cdot 10^{11}, c_{66}=1.19 \cdot 10^{11}, c_{12}=1.95 \cdot 10^{11}, c_{13}=1.25 \cdot 10^{11}\right][\mathrm{Pa}]$ with a density of $\rho=3100\left[\mathrm{~kg} / \mathrm{m}^{3}\right]$ [177]. Finally, the parameters describing the silica substrate were a Young's modulus $=73.1[\mathrm{~Pa}]$, a Poisson's ratio of $\nu=0.17$ and a density $\rho=2203$ $\left[\mathrm{kg} / \mathrm{m}^{3}\right]$, as in Chapter 5. In addition to the optical and mechanical material properties, also the photoelastic coefficients must be taken into account. The photoelastic constants in contracted notation were $\left[p_{11}=-0.09, p_{12}=0.017, p_{44}=-0.051\right]$ for silicon [81] and $\left[p_{11}=0.121, p_{12}=0.27, p_{44}=-0.075\right]$ for the silica substrate and the silicon nitride layer. Notice that the photoelastic coefficients of the $\mathrm{Si}_{3} \mathrm{~N}_{4}$ were assumed to be equal to those of silica as they have been largely unknown in the literature [178] though a recent experiment has reported an experimental value $p_{12}=0.047$ [174].

The photonic dispersion band diagram of the engineered configuration, obtained with RSoft BandSolve [94], is presented in Fig. 6.2(a). Here, it can be seen that by properly choosing the dimensions of the system, with $w=325 \mathrm{~nm}, b=0.9 w, e=450 \mathrm{~nm}$ and $t=600$ $\mathrm{nm}$, in this case, the silicon core can support a set of confined optical guided modes. This may also be the case for other dimensions of the system. The proposed dimensions consist in a optimized set of values obtained once performing the analysis presented in this chapter. Here, as it was discussed in Section 2.2.1, we can distinguish TE-like and TM-modes as a function in the relevant telecom band, where the involved wavevectors of those two modes are also presented in the figure. An inset view of the total electric field of those field profiles is also presented for the sake of completeness. Touching the mechanical properties, the phononic band diagram dispersion can also be obtained and it is shown in Fig. 6.2(b). It is noteworthy that as now the system is not isolated from the substrate, a lot of leakage mechanical modes can appear in the simulations, thus requiring post-processing of the confined mechanical modes under interest. Because of that, the mechanical dispersion relation shows all the modes that we can find in the structure and the color scale represents the acoustic localization factor in the silicon core, calculated as 
(a)
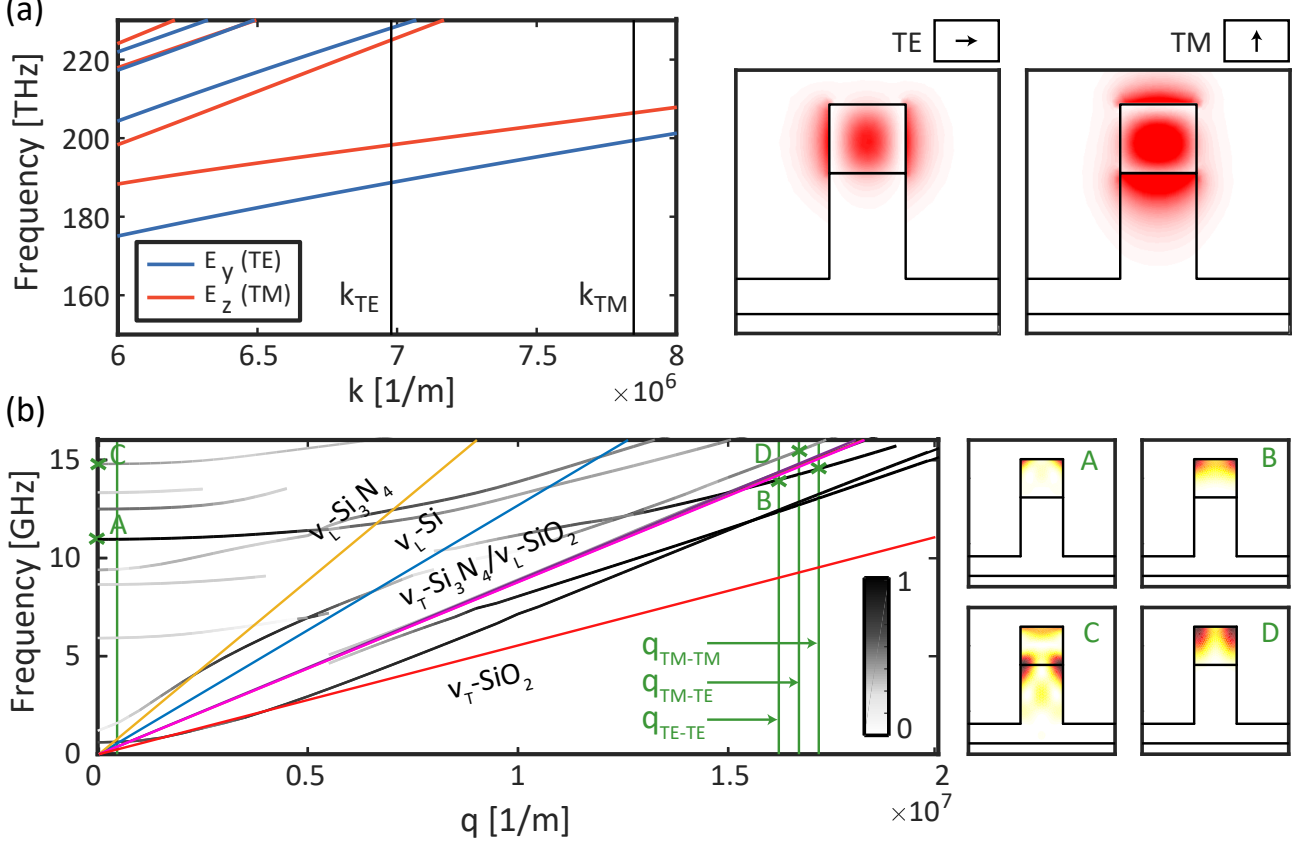

Figure 6.2: (a) Calculated dispersion relation of the TE-like and TM-like optical guided modes with the optical wavevectors involved for each mode. Insets: Computed $E_{y}$ and $E_{z}$ components of the optical TE-like and TM-like waveguide modes, respectively. (b) Calculated dispersion relation for the mechanical modes. The interfaces between $\mathrm{Si} / \mathrm{Si}_{3} \mathrm{~N}_{4}$ and air are treated as free boundaries and perfectly matched layer conditions are imposed at the substrate limits interfaces. Here, the colorscale shows the acoustic localization factor of the mechanical mode within the silicon core (this factor is 1 for a mechanical mode completely confined in the silicon core). Insets: Mechanical mode profiles for the different phase matching configuration conditions. The dimensions of the waveguide under consideration are $w=325 \mathrm{~nm}, b=0.9 w, e=450 \mathrm{~nm}$ and $t=600 \mathrm{~nm}$.

$\left(\int_{S_{1}}|\mathbf{u}|^{2} d S\right) /\left(\int_{S_{2}}|\mathbf{u}|^{2} d S\right)$ where $u$ is the total mechanical mode displacement, and $S 1$ and $S 2$ denote the silicon core and the full cross-section under consideration, respectively. Notice that this definition is equivalent to that used for the localization factor in Chapter 5. Darker lines in this plot represent a localization close to unity, which means that the mechanical mode is well located in the silicon region. In contrast, lighter lines show less localized modes. In Fig. 6.2(c) the mechanical modes with the highest Brillouin gain for different intramodal and intermodal configurations have been highlighted. The mechanical modes termed $\mathrm{A}$ and $\mathrm{C}$ give the highest $G_{B}$ values in the case of forward intramodal TE and TM coupling, respectively. The mechanical mode B is involved in both backward intramodal TE-TE and TM-TM interaction whilst the mechanical mode $\mathrm{D}$ is the one that gives a higher $G_{B}$ value in backward intermodal TE-TM SBS. However, a close view of these displacement profiles can be seen in the next sections.

From the photonic and phononic dispersion diagrams, we have seen that both optical and mechanical modes can be found in the structure under study. However, in order to understand and calculate the characterizing parameter of the strength of the acoustooptic interaction between those modes, a close view of the theory must be taken. 


\subsection{AO interaction and Brillouin gain}

Concerning the AO interaction in the stimulated Brillouin scattering process, propagating optical and mechanical modes interact via volumetric and surface processes as long as energy and momentum are conserved $[61,82,98]$. In an AO waveguiding system as the one presented here, $G_{B}$ for a certain mechanical mode can thus be obtained as [81]:

$$
G_{B}=\frac{2 \omega Q_{m}}{\Omega_{m}^{2}} \frac{\left|\left\langle\mathbf{f}, \mathbf{u}_{m}\right\rangle\right|^{2}}{\left\langle P_{p}\right\rangle\left\langle P_{s}\right\rangle\left\langle\mathbf{u}_{m}, \rho \mathbf{u}_{m}\right\rangle}
$$

where $m$ is the mechanical mode number (omitted in what follows for the sake of simplicity), $\left\langle u_{m}, \rho u_{m}\right\rangle$ is the mechanical mode effective mass, $\langle P\rangle=\frac{1}{2} v_{g}\langle\mathbf{E}, \epsilon \mathbf{E}\rangle$ is the averaged Poynting vector for guided pump (" $p ")$ and Stokes (" $s$ ") waves, $\mathbf{E}=$ $\left(\left(\mathbf{E}_{p} e^{i\left(-k_{p} x+\omega_{p} t\right)}+\mathbf{E}_{s} e^{i\left(-k_{s} x+\omega_{s} t\right)}\right) / 2+\right.$ c.c. $)$ is the electric field, $\mathbf{D}=\epsilon \mathbf{E}$ is the displacement field, $\epsilon$ is the material permittivity, $v_{g}$ is the group velocity, $\mathbf{f}$ is the optical force, $\mathbf{u}=u_{x} \mathbf{x}+u_{y} \mathbf{y}+u_{z} \mathbf{z}$ is the elastic displacement caused by the total optical power created by the pump and Stokes waves, and $\Omega_{m}$ and $Q_{m}$ are the frequency and the mechanical quality factor of the involved mechanical mode at $\mathbf{q}=\mathbf{k}_{p}-\mathbf{k}_{s}$. As the propagating optical waves are considered to evolve in the same direction, the last expression can be simplified in the propagation direction as $q=k_{p} \pm k_{s}$ as a function of their co- or counter-propagation.

Notice that in the calculations performed of the results shown from now on a mechanical Q factor $Q_{m}=10^{3}$ was assumed, meaning that the mechanical damping is limited by material losses [142]. This is a typical convention followed in cavity and waveguide optomechanics at $\mathrm{GHz}$ frequencies when operated at room temperature. However, in our calculations below we kept the original mechanical quality factor for modes showing $Q_{m}<10^{3}$ in simulations, in order to not overestimate leaky modes.

Then, as a result of the continuous translational symmetry along the propagation axis, the overlapping between the optical and mechanical fields in Eq. 6.1 is defined by the inner product between two vector fields with integration over the whole waveguide cross-section, $S$ :

$$
\langle\mathbf{A}, \mathbf{B}\rangle=\int_{S}\left(\mathbf{A}^{*} \cdot \mathbf{B}\right) d S
$$

We consider the total optical force $\mathbf{f}$ exerted by pump and Stokes waves and originating from electrostriction bulk and interface forces as well as radiative pressure [81]. The elastodynamics equation, including the electrostrictive stress induced by the optical field, reads as [95]:

$$
\rho \frac{\partial^{2} u_{i}}{\partial t^{2}}-\left[c_{i j k l} u_{k, l}\right]_{j}=-\sigma_{i j, j}^{e s}
$$

where $c_{i j k l}$ is the rank- 4 tensor of elastic constants, $\sigma_{i j}^{e s}=-\frac{1}{2} \epsilon_{0} \chi_{k l i j} E_{k} E_{l}$ is the electrostrictive stress tensor, with the rank- 4 susceptibility tensor $\chi_{k l i j}=\epsilon_{k m} \epsilon_{l n} p_{m n i j}\left(\sigma_{i j}^{e s}=\right.$ $\left.-\frac{1}{2} \epsilon_{0} \epsilon_{k m} \epsilon_{l n} p_{m n i j} E_{k} E_{l}\right), p_{m n i j}$ is the photoelastic tensor, and $\epsilon_{0}$ is the permittivity of vacuum.

Finally, the overlapping between optical and mechanical fields in Eq.(1) is defined as 


$$
\begin{aligned}
\langle\mathbf{f}, \mathbf{u}\rangle=\iint_{S} d S\left(\mathbf{f}^{E S, *} \cdot \mathbf{u}\right) & +\oint_{\partial S} d l\left(\mathbf{F}^{E S, *} \cdot \mathbf{u}\right) \\
& +\oint_{\partial S} d l\left(\mathbf{F}^{R P, *} \cdot \mathbf{u}\right)
\end{aligned}
$$

where $f^{E S}=-\nabla \widehat{\sigma}$ is the electrostriction (bulk) force, $\mathbf{F}^{E S}=\left(\widehat{\sigma}^{(1)}-\widehat{\sigma}^{(2)}\right) \cdot \mathbf{n}$ is the electrostriction interface force and $\mathbf{F}^{R P}=\left(\widehat{T}^{(1)}-\widehat{T}^{(2)}\right) \cdot \mathbf{n}$ is the radiative pressure, $\mathbf{n}$ being the normal to the boundary between contacting media. Here the index (1) describes the internal medium and (2) describes the outward medium of the interface. The radiative pressure is defined by the Maxwell stress tensor, $T_{i j}=\varepsilon_{0} \varepsilon\left(E_{i} E_{j}-\frac{1}{2} \delta_{i j} E^{2}\right)$, and can be reduced to the expression:

$$
\mathbf{F}^{R P}=\frac{1}{2}\left(\varepsilon_{0}\left(\varepsilon_{1}-\varepsilon_{2}\right) E_{p t} E_{s t}^{*}-\varepsilon_{0}^{-1}\left(\varepsilon_{1}^{-1}-\varepsilon_{2}^{-1}\right) D_{p n} D_{s n}^{*}\right) \cdot \mathbf{n}
$$

\subsection{Intramodal first order mode interaction}

From the derivation of the Brillouin gain through the analysis of the overlapping between the two optical fields and the generated acoustic wave, different configurations can be now addressed in order to obtain a high $G_{B}$. According to Fig. 1.4 different situations were possible both involving intramodal or intermodal interactions of even forward or backward propagations. However, in this chapter, we will focus only on intramodal interactions as, for this practical application, it was obtained that intermodal interactions did not result in high coupling rates. It must be added that all the simulations regarding the SBS process were performed by using the Livelink platform between COMSOL Multiphysics and Matlab.

\subsubsection{Forward Stimulated Brillouin Scattering}

Forward SBS takes place when the pump and Stokes waves propagate co-directionally. Then, the momentum conservation implies that the generated acoustic phonons will have a mechanical wavevector given by $q=k_{p}-k_{s}$. As we are exploring intramodal coupling, that means that both the pump and the Stokes waves have the same optical field profile which involves that they have also the same optical wavevector. Then, the obtained mechanical modes are found at $q=0$ thus resulting in completely guided modes because phonons cannot escape from the silicon core. Therefore, large values of $G_{B}$ in Forward Stimulated Brillouin Scattering (FSBS) processes have been obtained by simulations [81] and confirmed experimentally [159]. When the silicon - or $\mathrm{Si}_{3} \mathrm{~N}_{4}$ - core is on top of a silica substrate, there is no way to confine the phonons there since the sound velocity in silica substrate is lower than in the core. In our system, even though mechanical modes at $q \approx 0$ (required for FSBS) are leaky (or, in other words, they do not have an infinite mechanical Q factor ideally), they can still be largely confined inside the silicon core, as shown in Fig. 6.2(c). This is because the intermediate $S i_{3} N_{4}$ layer partially prevents the leakage of phonons into the silica substrate. Therefore, we performed numerical calculations to evaluate $G_{B}$ for the FSBS process in two situations: intramodal interaction between either TE or TM optical modes.

The results for intramodal TE FSBS are summarized in Fig. 6.3. Here, we can observe the profiles of the optical forces obtained from bulk and interface electrostrictive 
(a)

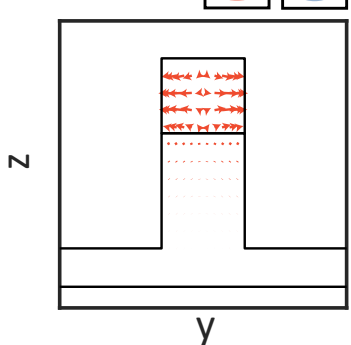

(b)

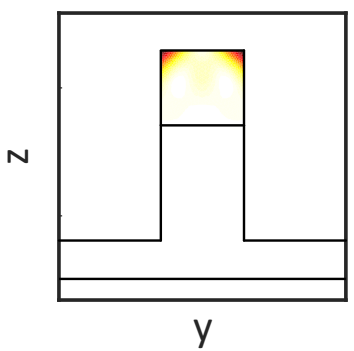

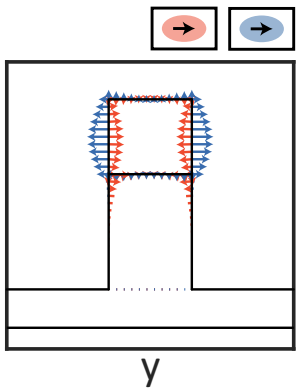

(c)

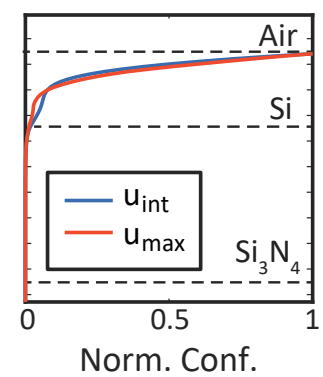

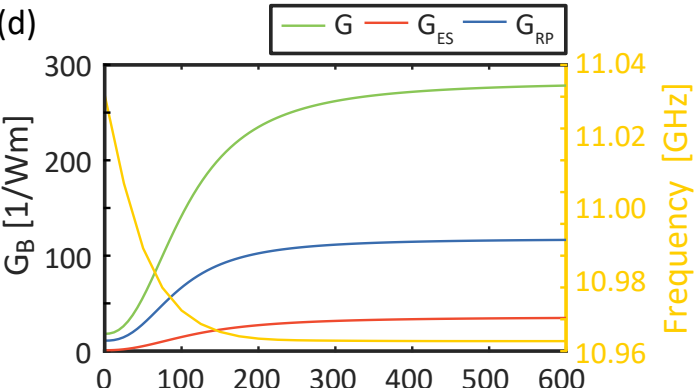

(e)

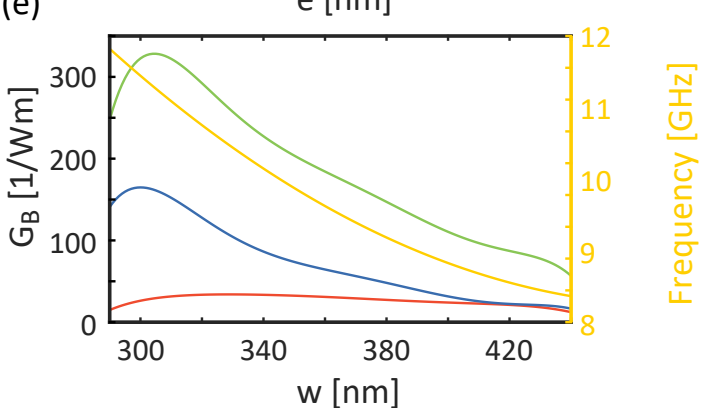

Figure 6.3: Intramodal TE FSBS interaction at $q=0$. (a) Optical force distributions arising from the interaction of two co-propagating TE-like modes (Stokes and pump waves). The first panel shows the bulk electrostrictive force (red) and the second panel shows the electrostrictive (red) and radiation pressure (blue) interface forces. (b) Total displacement of the mechanical mode with the highest $G_{B}$ (termed A in Fig. 6.2(c)). (c) Normalized confinement distribution of the total accumulated displacement (blue) and maximum displacement (red). (d) Total Brillouin gain $G_{B}$ (green) and its electrostrictive (red) and radiation pressure (blue) contributions as a function of the etching depth $e$ of the $S i_{3} N_{4}$ layer for a structure with dimensions $w=325 \mathrm{~nm}, b=0.9 w$ and $t=600 \mathrm{~nm}$. (e) Total Brillouin gain $G_{B}$ (green) and its electrostrictive (red) and radiation pressure (blue) contributions as a function of waveguide width $w$ for $e=450 \mathrm{~nm}$ and $t=600 \mathrm{~nm}$. In (d) and (e) the mechanical frequency is depicted in yellow.

(red) and radiation (blue) pressure effects (Fig. 6.3(a)). For these force profiles, the mechanical mode termed A in Fig. 6.3(c) gives the highest gain. The displacement of this mechanical mode, which has an acoustic localization factor of 0.989 and a theoretical $\mathrm{Q}$ factor - this is, neglecting material losses - of $2 \times 10^{5}$, is depicted in Fig. 6.3(b), where it can be appreciated that the displacement field is mainly located at the top interface of the silicon core. The strong localization in the silicon core is confirmed by calculating the accumulated mechanical displacement, obtained as $u_{\text {int }}=\int_{-w / 2}^{w / 2}|u|^{2} d y$, and the maximum displacement as a function of the height, as depicted on Fig. 6.3(c). This leads to relatively large $G_{B}$ values $\left(G_{B, T E} \approx 3001 / \mathrm{Wm}\right)$ for the mechanical mode A as long as the etching $e$ is deeper than $250 \mathrm{~nm}$ (Fig. 6.3(d)). Indeed, the general trend is that the Brillouin gain grows with $e$ and finally saturates when there is full etching of the silicon nitride layer $(e=t)$. When calculating the Brillouin gain as a function of $w$, we can see that there is a waveguide width that maximizes the gain, as shown in 6.3(e).

Importantly, changing the waveguide width also allows us to tune the mechanical frequency $f_{m}$. As shown in Fig. 6.3(e), changing the waveguide width from 290 to 450 
nm tunes $f_{m}$ between $\approx 8 \mathrm{GHz}$ and $\approx 12 \mathrm{GHz}$. This is an important feature because it may allow tuning the Brillouin frequency across the whole $\mathrm{X}$ band, which is very important in many microwave applications. As the mechanical mode is mainly confined on the edges of the upper surface of the silicon region, as can be appreciated in Fig. 6.3(b), the main contribution to the total Brillouin gain is given by the radiation pressure force at that interface. Regarding the electrostrictive contribution, it has to be noted that the field profiles of the bulk and interface contributions have opposite directions, resulting in destructive interference of the total contribution. Nevertheless, the radiation pressure and the total electrostrictive contributions interfere constructively for this configuration, leading to the relatively large calculated values of the Brillouin gain.

(a)

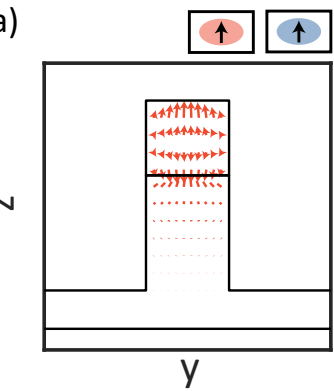

(b)

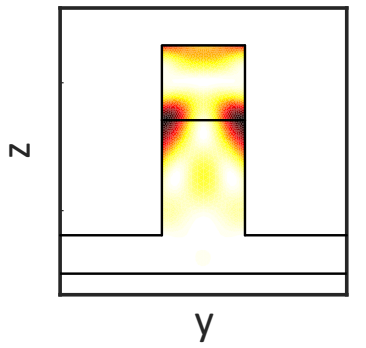

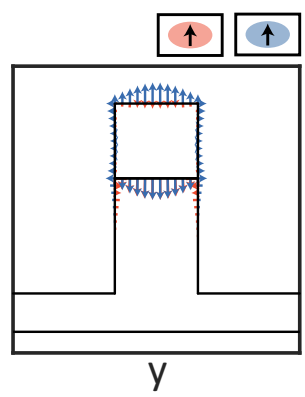

(c)

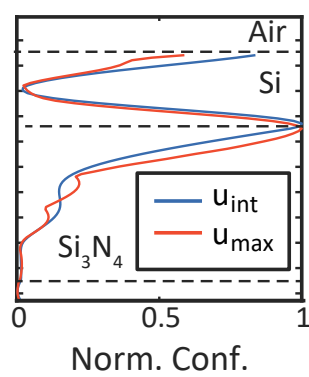

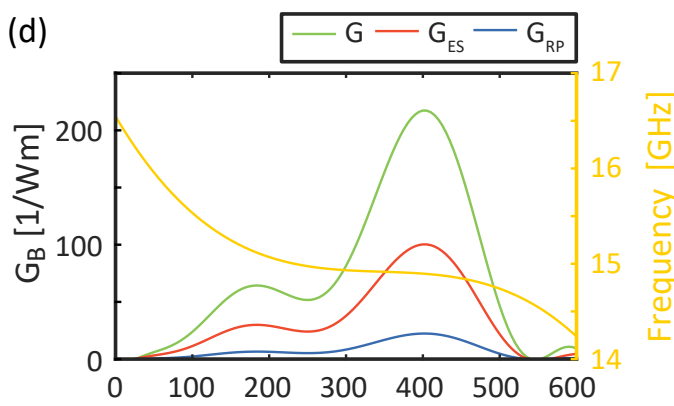

(e)

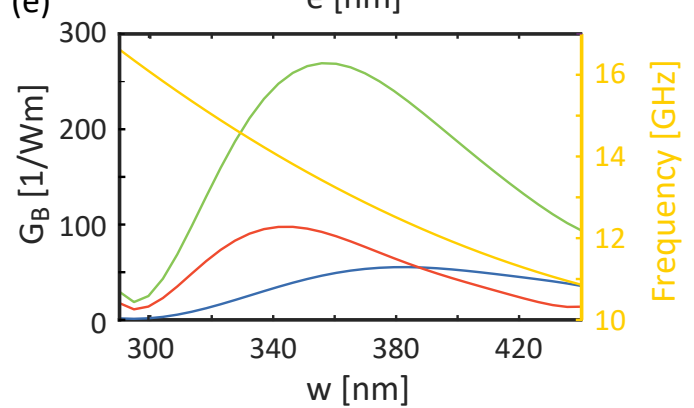

Figure 6.4: Intramodal TM FSBS interaction at $q=0$. (a) Optical force distributions arising from the interaction of two co-propagating TM-like modes (Stokes and pump waves) for FSBS. The first panel shows in red the bulk electrostrictive force and the second panel shows the electrostrictive (red) and radiation pressure (blue) interface forces. (b) Calculated displacement of the mechanical mode with the highest $G_{B}$ (termed $\mathrm{C}$ in Fig. 6.2(c)). (c) Normalized distribution of the total accumulated displacement (blue) and maximum displacement (red) as a function of the height of the structure. (d) Total $G_{B}$ gain (green) and its electrostrictive (red) and radiation pressure (blue) contributions as a function of the etching depth of the $\mathrm{Si}_{3} N_{4}$ layer for a structure with dimensions $w=325 \mathrm{~nm}, b=0.9 w$ and $t=600 \mathrm{~nm}$. (e) Total $G_{B}$ (green) and its electrostrictive (red) and radiation pressure (blue) contributions as a function of waveguide width $w$ for $e=450$ $\mathrm{nm}$ and $t=600 \mathrm{~nm}$. In (d) and (e) the mechanical frequency is depicted in yellow.

The results for intramodal TM FSBS are summarized in Fig. 6.4. Firstly, as in the previous case, the obtained optical field profiles are shown in Fig. 6.4(a) where it can be appreciated, for example, that the bulk electrostrictive force and the interface radiation pressure have opposite directions than in the forward intramodal TE configuration. Again, we obtain relatively large values of $G_{B}$ (Fig. 6.4(d)), peaking close to $3001 / \mathrm{Wm}$. 
As in the previous case, we also observe that changing the waveguide width $w$ allows us for tuning the mechanical frequency and optimizing the $\mathrm{AO}$ coupling. Indeed, the best configuration can be found for an $e \approx 400 \mathrm{~nm}$ and $w \approx 360 \mathrm{~nm}$, with a mechanical frequency $f_{m} \approx 13 \mathrm{GHz}$. Here, the main contribution to the total gain comes from the electrostrictive effect, for which the bulk and interface field profiles have now the same direction in the region where the mode is localized. The main difference with respect to the TE case is that the mechanical mode that couples with the forces distributions (Fig. 6.4(b)) in this configuration is not so well confined in the silicon core as before. This relatively poor confinement can also be observed in Fig. 6.4(c), where it is seen that part of the mechanical displacement is in the pedestal region, as well as from the calculation of the acoustic localization factor, which is now $\approx 0.5$, and the calculated mechanical quality factor, which is $10^{2}$. In this case, as the calculated $Q_{m}$ is lower than $10^{3}$, the upper limit due to material losses at room temperature, we used $Q_{m}=10^{2}$ in the calculations of the Brillouin gain.

\subsubsection{Backward Stimulated Brillouin Scattering}

The process of Backward Stimulated Brillouin Scattering (BSBS) involves large $q$ mechanical modes that should be ideally placed below the sound line of the silica substrate in order to completely suppress mechanical leakage. Additionally, mechanical modes over the sound line could be well confined in the silicon core and give rise to strong AO interaction and large Brillouin gain, as above in the case of FSBS. As in the last section, we explored two different backward configurations for an intramodal coupling of TE-TE and TM-TM modes. The obtained optical force profiles resulting from that interaction and the driven mechanical modes are presented in Fig. 6.5. Especially, the bulk and interface forces (Fig. 6.6(a)) as well as the mechanical mode profile high the highest SBS gain (Fig. 6.6(b)) and its vertical confinement (Fig. 6.6(c)) are presented in the first row of the figure for the TE-TE case. Concerning the TM-TM situation, Figures 6.6(d) to (f) show the same magnitudes. From those mechanical modes we can observe that even at different matching wavevectors, one is at $q=2 k_{T E}$ and the other at $q=2 k_{T M}$, the acoustic field profiles look remarkably similar.

With reference to the obtained $G_{B}$ values, in our study, we considered first intramodal BSBS resulting from the interaction of two counter-propagating TE-like optical modes and select again the mechanical mode providing a larger $G_{B}$ (in the case, the mode B in Fig. 6.2(c)). We get $G_{B} \approx 3401 / \mathrm{Wm}$ at frequencies $f_{m} \approx 12.4 \mathrm{GHz}$, as can be seen in Fig. 6.5(a). In this configuration, the electrostrictive contribution in the total gain is nearly zero. This results from the destructive interference between both the bulk and the interface forces that, as seen in Fig. 6.5(a), are opposite. Besides that, we get a mechanical mode highly localized in the silicon core (Fig. 6.5(b) and (c)). In this case, the calculated mechanical quality factor is $2.8 \times 10^{7}$, much larger than in the forward configuration. This could be explained by the fact that, unlike the modes at $q \approx 0$, this mechanical mode is placed below the longitudinal speed of sound in silica, as observed in Fig. 6.2(c). Regarding the acoustic localization factor, we get a value of 0.9993 , in accordance with a high localized mechanical mode.

In the case of backward intramodal TM configuration (Fig. 6.6(b)), we observe that unlike in the intramodal TE BSBS case, now the electrostriction contribution gain reaches values around $6001 / \mathrm{Wm}$. However, the radiation pressure and electrostriction contributions interfere destructively, thus resulting in $G_{B} \approx 2201 / \mathrm{Wm}$ for the mode 
(a)

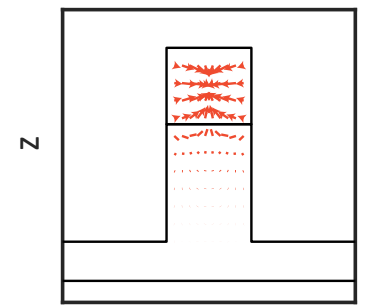

y

(d)

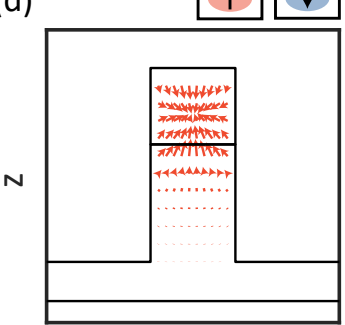

y

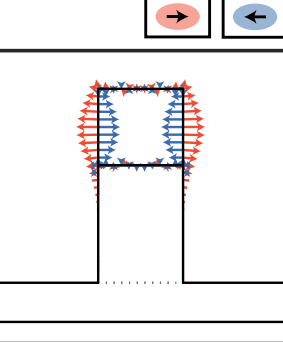

y

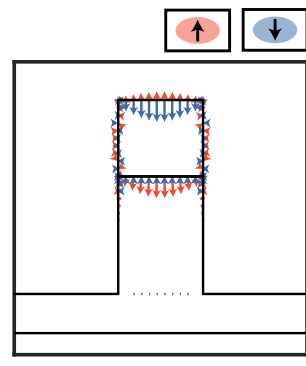

y (b)

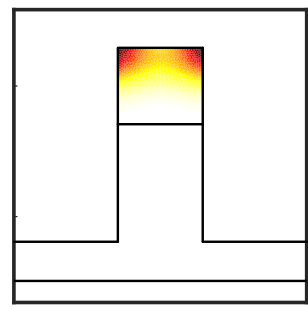

y

(e)

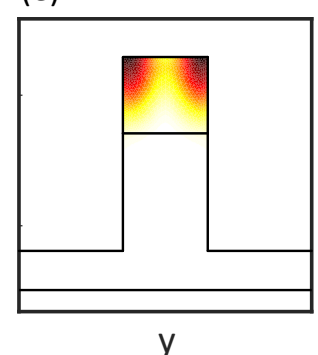

(c)

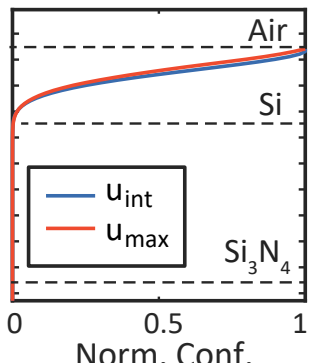

(f)

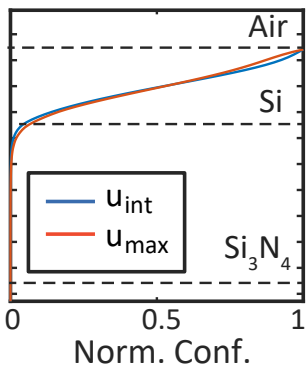

Figure 6.5: Intramodal TE and TM BSBS interaction at $q=2 k_{T E}$ and $q=2 k_{T M}$. (a) Optical force distributions arisen from the interaction of two counter-propagating TElike modes. The first panel shows the bulk electrostrictive force whilst the second panel shows the electrostrictive (red) and radiation pressure (blue) interface forces. (b) Total mechanical displacement of the mode with the highest $G_{B}$ (termed B in Fig. 6.2(c)). (c) Normalized distribution of the total accumulated displacement (blue) and maximum displacement (red). (d) Optical field distributions from the interaction of two counterpropagating TM-like modes. (e) Total mechanical displacement of the mode with the highest $G_{B}$ for the counter-propagating case. (f) Normalized distribution of the total accumulated displacement (blue) and maximum displacement (red) of the mode termed D in Fig. 6.2(c).

termed B in Fig. 6.2(c) (see the displacement profile in Fig. 6.5(e)). Nevertheless, as the mechanical mode has a large acoustic localization factor (0.9995) as well as an ultrahigh mechanical quality factor $\left(Q_{m}=2.3 \times 10^{7}\right)$ there is a slight improvement in the total gain when the etching depth increases. Still, these values could be sufficient for practical applications. It must be noted that in the two intramodal configurations $G_{B}$ increases the more $\mathrm{Si}_{3} \mathrm{~N}_{4}$ is etched. In fact, despite just the presence of the silicon nitride layer enables the mechanical confinement of the mode with $e=0 \mathrm{~nm}$, a totally etched layer with $e=600 \mathrm{~nm}$ results in the highest performance.

Additionally, we also calculated the Brillouin gain for intermodal TE-TM interaction. Even though the results are not shown here for the sake of simplicity, the calculated values were lower than in the case of intramodal interactions. This can be probably due to the fact that the mode that gives the highest gain for this configuration at $q=k_{T M}-k_{T E}$ is the mode termed D in Fig. 6.2(c), which has a localization poorer than the other mechanical modes even at high wavevectors. Still, further design of the waveguide may result in large $G_{B}$ values for intermodal interaction. 
(a)

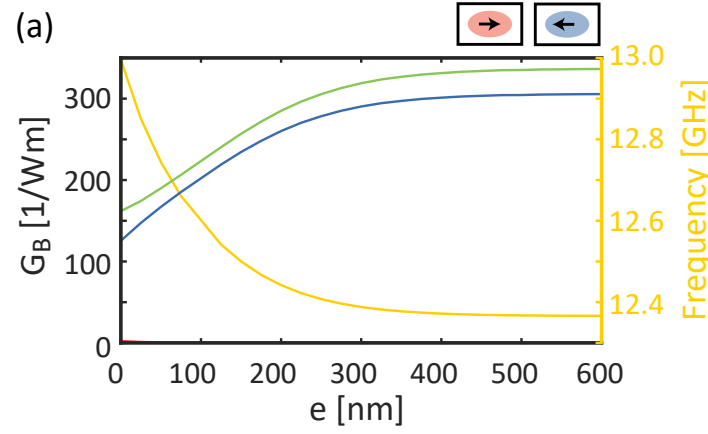

(b)

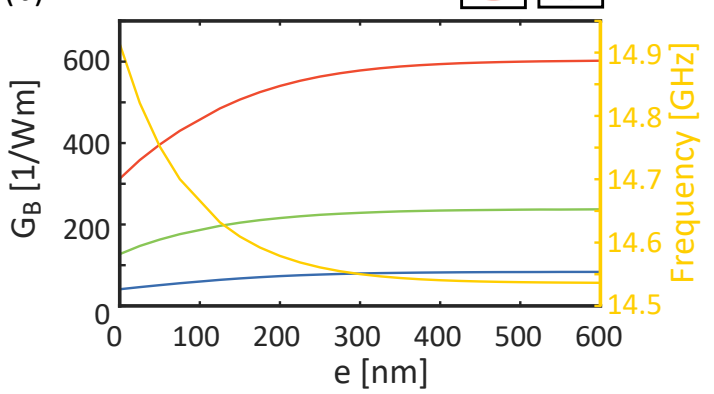

Figure 6.6: (a) Total intramodal TE SBS gain (green) and its electrostrictive (red) and radiation pressure (blue) contributions as a function of the etched layer $e$. The mechanical frequency evolution is depicted in yellow. (b) Total intramodal TM SBS gain (green) and its electrostrictive (red) and radiation pressure (blue) contributions as a function of the etched layer $e$. The mechanical frequency evolution is depicted in yellow. In both panels the mechanical frequency evolution is depicted in yellow.

\subsection{Enhancement of the Brillouin gain at low temperature}

As we noted in previous sections, the mechanical Q factor was assumed to be $Q_{m}=10^{3}$ because the mechanical damping is limited by the material loss at room temperature. However, this is not the case if we work at low temperatures, where $Q_{m}$ factor can be enhanced up to much higher values [142]. If we study the evolution of the ideal mechanical quality factor (this is, as obtained from the simulations and neglecting material losses) of the mechanical modes involved in our configurations, we can see that it grows as the etching of the $\mathrm{Si}_{3} \mathrm{~N}_{4}$ layer $e$ is increased up to its maximum (which is the maximum thickness of the silicon nitride layer, $t=600 \mathrm{~nm}$ ). This effect is clearly seen in Fig. 6.7(a) for the case of the intramodal TE FSBS interaction. Here, we can see also the evolution of the acoustic localization factor (in yellow) in the silicon core as a function of $e$. It can be appreciated that the mechanical mode is almost totally confined (the localization factor is almost 1) regardless of the etching depth $e$ and it is only reduced in the case where there is not any pedestal and the silicon waveguide lies on a $\mathrm{Si}_{3} \mathrm{~N}_{4}$ substrate. The modified low-temperature Brillouin gain, taking into account the ideal $Q_{m}$ and introducing it in Eq. 6.1, is shown in Fig. 6.7(b). This means that for the same situation like the one described in Fig. 6.3(d) the total Brillouin gain is increased from $3001 / \mathrm{Wm}$ up to $1.5 \times 10^{5} 1 / \mathrm{Wm}$, which should be attainable when operating at cryogenic temperatures. It must be remarked that all the results presented in this chapter can also be found at Ref. [179].

\subsection{Practical implementation}

The AO performance of the waveguiding structure under study and, in particular, its ability to confine mechanical waves in the silicon core, are extremely dependent on the mechanical behavior of the intermediate $\mathrm{Si}_{3} \mathrm{~N}_{4}$ layer. In our calculations, we have modeled the $\mathrm{Si}_{3} \mathrm{~N}_{4}$ layer assuming it is crystalline and following the parameters reported in [177]. Noticeably, the mechanical properties of $\mathrm{Si}_{3} \mathrm{~N}_{4}$ will change slightly depending 

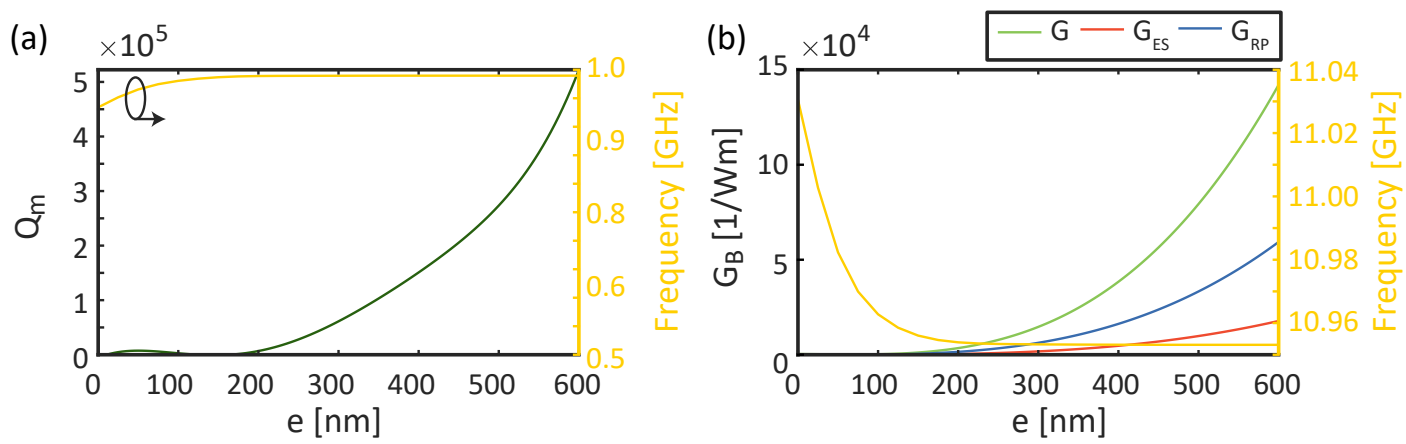

Figure 6.7: Intramodal TE FSBS interaction at $q=0$ at low temperature (assuming that mechanical damping is not limited by material losses). (a) $Q_{m}$ (purple) and acoustic localization factor (yellow) as a function of $e$. (b) Brillouin gain as a function of the $e$ for $w=325 \mathrm{~nm}, b=0.9 w$ and $t=600 \mathrm{~nm}$ calculated using the ideal $Q_{m}$ factor in (a).

upon whether the material is either crystalline or amorphous, as well as depending on the crystal lattice orientation for crystalline $\mathrm{Si}_{3} \mathrm{~N}_{4}$ [180] or the deposition process in the case of amorphous $\mathrm{Si}_{3} \mathrm{~N}_{4}$. Typically, $S i_{3} N_{4}$ layers used in integrated photonics are deposited using low-pressure chemical vapor deposition (LPCVD), since this method enables low propagation losses at telecom wavelengths [181]. Interestingly, $70 \mathrm{~nm} \mathrm{Si}_{3} \mathrm{~N}_{4}$ layers deposited using LPCVD exhibit sound speeds quite similar to the ones we have used in our calculations [182]. This means that the intermediate layer of our waveguiding structure could be in principle built by depositing an amorphous silicon nitride layer on top of a silica substrate using LPCVD.

Then the silicon core could be bonded in crystalline state on top of the $\mathrm{Si}_{3} \mathrm{~N}_{4}$ layer or in nanocrystalline-state by using chemical vapor deposition and annealing. Noticeably, thin layers of nanocrystalline silicon exhibit optical and mechanical losses similar to crystalline samples $[183,184]$. Later, the whole multilayer system could be processed using electron beam lithography and dry etching. Therefore, our system would be implementable in practice using standard silicon technology. Indeed, since optical losses depend mainly on sidewall scattering due to etching-induced roughness, we could expect optical propagation losses similar to the case of rectangular-core silicon waveguides [159].

In addition, one may think that removing the silica substrate would completely solve the problem of mechanical leakage. This could be done by assuming a semi-infinite silicon nitride substrate on top of which the silicon core is defined. However, this solution is quite unrealistic because it is technologically challenging to build thick ( $\geq 1 \mu \mathrm{m}$ ) $\mathrm{Si}_{3} \mathrm{~N}_{4}$ layers that do not break due to tensile stress. Another choice would consist of replacing the silica substrate by silicon. However, the optical waves would not be fully confined in the upper silicon core due to the high index of the silicon substrate unless the $\mathrm{Si}_{3} \mathrm{~N}_{4}$ layer was very thick, which would face the same fabrication issues mentioned previously. 


\section{Chapter 7}

\section{Conclusions and perspectives}

In this thesis, it has been shown that optomechanical crystal cavities can be a key building block in all-optical microwave processing mediated by phonons. Phonon manipulation in these acousto-optic interacting systems can pave the way towards the development of ultracompact RF processing components in the optical regime, thus avoiding the signal processing in the electrical domain. Concerning this subject, several main goals have been achieved in this field:

- First of all, a novel silicon OM crystal cavity that exhibits a large OM coupling rate for a $\mathrm{GHz}$ mode within a full phononic bandgap was designed, fabricated and demonstrated experimentally. More importantly, this cavity was experimentally demonstrated to act as an ultracompact OM microwave oscillator at room temperature for a microwave frequency in the top range of the $\mathrm{S}$ band $(2-4 \mathrm{GHz})$. In that regard, an essential parameter describing the performance of the OM oscillator the phase noise - was measured, showing a value as low as $-100 \mathrm{dBc} / \mathrm{Hz}$ at 100 $\mathrm{kHz}$. Noticeably, this value is on par with commercial mid-range devices which values of $-102 \mathrm{dBc} / \mathrm{Hz}$ at the same frequency offset [113].

- Moreover, as a consequence of the nonlinear behavior of the OMC, it was possible to drive the system well above the lasing threshold to generate an OFC of OM nature. Under this situation, multiple harmonic generation was obtained up to the 6th harmonic, thus reaching the $20 \mathrm{GHz}$ range. That means the generation of multiple RF tones where the functionality of the first harmonic can be extrapolated to those high frequencies. The phase noise value for higher harmonics was also demonstrated to degrade as in conventional harmonic mixing but even then we were able to show noise figures below $-80 \mathrm{dBc} / \mathrm{Hz}$ at $100 \mathrm{kHz}$ above $15 \mathrm{GHz}$. The preliminary demonstration of the OFC paves the way towards the synthesis of microwave signals beyond the generation of pure continuous wave tones. The main advantages of the OM cavity approach for $\mathrm{RF}$ and microwave signal processing are its extreme compactness and low weight, highly desirable in space and satellite applications, and its compatibility with silicon electronics and photonics technology. The resulting optomechanically generated OFC could also be useful in sensing and spectroscopy applications by taking profit of the small distance between comb lines which would enable detection using standard electronic equipment.

It must be noted, however, that our device is a free-running or open-loop oscillator, which means that microwave tone generated by the device is not attained by any feedback 
loop which, besides, could improve its frequency stabilization. Adding such a feedback loop should improve even more the OMO performance, thus being more competitive. Also, operating at cryogenic temperatures would enormously improve the phase noise [42] as a result of the enhancement of the mechanical Q factor because of the full phononic bandgap [89]. As a remark, this new OM cavity could be useful in quantum applications since the mechanical Q factor can be extremely increased in cryogenic environments [185]. This would also allow us to discern if the phononic bandgap plays a role in the better performance in terms of phase noise in comparison to the OM crystal reported in [44]. Tunability of the resulting microwave signal could be achieved by injection locking to an external optically-modulated tone [186].

Our result confirms that OM cavities can be used to synthesize microwave waveforms beyond the generation of single microwave tones and, therefore, they can play a role in the development of microwave photonics applications in silicon photonics technology. It could be disruptive in the way towards extreme miniaturization and on-chip integration of such components [126], since no other approach enables photonics-based microwave processing in wavelength-scale foot-prints [40]. Note that the total cavity length is around $10 \mu \mathrm{m}$. This fact, added to the flexibility to tune the fundamental mechanical frequency by an appropriate design of the cavity [92], would enable to adopt this approach in a wide variety of microwave systems and networks enabled by all-optical processing in silicon chips. Besides that, as a step further:

- We have demonstrated experimentally that OM crystal cavities can be used successfully as nonlinear elements capable to perform down- and up-conversion of digitally modulated signals employing OFDM modulation at frequencies around $4 \mathrm{GHz}$ with $\mathrm{MHz}$-bandwidths. This functionality would enormously simplify the optoelectronic hardware in wireless systems since the OM cavity acts simultaneously as a mixing element and as a local oscillator. Remarkably, the whole process is coherent, which is demonstrated through the successful recovery of OFDM signals following the IEEE 802.16e WiMAX wireless standard after down- and upconversion. A frequency conversion efficiency greater than $-17 \mathrm{~dB}$ was obtained in both down- and up-conversion processes.

- The device, which is built on a silicon chip using standard microfabrication tools, can operate at the fundamental mechanical frequency as well as at integer multiples of it, so wireless systems operating at different spectral bands through the whole radio-frequency spectrum could be benefited from this approach. For instance, using the first harmonic, the up-conversion frequencies would span from $f_{m}-f_{m} / 2$ to $f_{m}+f_{m} / 2$. Therefore, our cavity would cover the regulated WiMAX bands at 3.5-3.7 $\mathrm{GHz}$ and $5.8 \mathrm{GHz}$, and would also be suitable for the $3.4-3.8 \mathrm{GHz}$ and 5.2-5.8 GHz commercial 5G new-radio (NR) bands [46]. Up-converted frequencies can be extended using higher-order harmonics: the second harmonic, measured at 7.8 GHz, would be suitable for 5G NR transmission in the mid-bands, as recently demonstrated (sub-7) [46] and the third harmonic measured at $11.7 \mathrm{GHz}$ reaches the technologically relevant X-band. Besides this, other OM cavities could also be used for frequency mixing at other frequencies. Indeed, OM crystals exhibit a tremendous degree of freedom in the design of the mechanical frequency, which can go from tens of $\mathrm{MHz}$ (using the flexural motion of the released silicon beam as in [90]) up to around $10 \mathrm{GHz}$ using more complex two-dimensional OM crystals [187]. Besides its use in photonics-enabled wireless communications systems, our work 
shows that OM cavities integrated into silicon chips hold the potential to produce a dramatic reduction in size, weight, and power consumption of microwave photonics devices, which is crucial in aircraft and satellite communication systems.

- As future perspectives, more complex data signals as 16QAM or 64QAM, which ensure higher data rates in communication networks employing OFDM, should be explored in these systems. Improving the signal to noise ratio of the free-running OMO via a feedback loop may ensure to fulfill the requirement limit of those configurations. Notice that the Floquet approach using an external modulation improves the phase noise though it requires multimode lasing behaviour.

All the previous results were obtained for a single OMC with one optical and a single mechanical mode. However, further functionalities may be obtained if increasing the number of mechanical oscillators confined in the cavity. Furthermore, they could enable other applications related to synchronization [47-53], stability enhancement [54] or dynamical topological phases [55]. With this perspective in mind;

- A proposal route to design multiple confine mechanical modes has been presented as well as an experimental confirmation of multiple confined mechanical modes in a single cavity. In addition, above threshold, a blue-detuned optical drive induces phonon lasing, which leads to the working regime of the last goals, it was important to demonstrate that multiple confined modes in a single structure can also get into this self-sustained regime. Hence, experimental single SSO states of both modes confined into the same structure were demonstrated and a mode competition behavior was obtained between them.

- However, the mode competition, as in regular "light" lasers [133], establishes a single mode limitation to get a multimode phonon laser. Therefore, in order to set two self-oscillating modes, thus overcoming this limit, an optical modulated input field was used to excite the cavity, based on a Floquet mode theory developed for this purpose [144]. Besides that, what we observe is that once we overcome the limit, employing an external modulated field set at a given frequency, the simultaneous phonon lasers get phase- or mode-locked. Here, by phase- or modelocked what we mean is the resulting state of locking these two waves (even at different frequencies). When this mode-locking is obtained for a given detuning from each other, by phase-locking the beat of the two to a stable reference (both signals at the beat frequency), one can also say that the lasers are phase-locked, even if they are oscillating at a different frequency.

- It is also observed that once the multimode lasing state is be reached experimentally, the long-term stability of the system is improved in comparison to the case of the single mode OMO. These results pave the way towards to integration of multiple coexisting $\mathrm{GHz}$ mechanical modes. The concepts we establish can be used to study the emergent physics of many-mode self-oscillating OM systems, in a fully controllable fashion.

Besides the demonstration of OM crystal cavities operating as RF processing elements in the optical domain using phonons, still some limitations can be found in those systems. One of them is related to the fact that despite the small foot-print of the OMC is very small and enables the miniaturization and multiple integration of cavities, the way 
to couple them simultaneously in an efficient manner needs to be improved. To couple a single OM cavity we use a fiber loop taper to selectively measure the cavity under study, as it has been broadened use for OMC characterization [11,31,51]. Other measurement approaches can also be found in the literature as coupling via free space [141], tapered coupling waveguides $[44,142,163]$ or grating couplers $[68,111]$. However, all these coupling systems only enable the simultaneous excitation of a reduced number of cavities. Because of that, other OM crystal cavities have been explored in order to enable multiple excitation of $\mathrm{OM}$ structures.

- Consequently, we have explored the field of high-index nanophotonics, which has been demonstrated in the past to hold quasi-bound states in the continuum (quasi$\mathrm{BIC}$ modes), resulting in relatively high values of the $\mathrm{Q}$ factor $(\mathrm{Q}>100)[57,58]$. Notably, the strong field localization in single disks extremely enhances nonlinear effects such as second [58] or third harmonic generation [148]. In this thesis, we have studied the OM interaction of those modes with their own mechanical oscillation modes where we obtained large values of $g_{0}$ that would ensure transduction of the GHz-scale mechanical modes as well as its manipulation either by cooling or heating them.

- In particular, blue-detuned laser driving at high power could ensure the formation of broad (i $100 \mathrm{GHz}$ ) optical frequency combs [57] in a wavelength-sized structure, opening the door towards ultra-compact microwave photonics. Notice also that the dimensions of the disks under study are smaller than those reported in $[156,158]$, which support high-Q optical and mechanical whispering gallery modes. This further miniaturization to sizes of the order of the wavelength allows for accessing mechanical modes at higher frequencies, going even beyond $10 \mathrm{GHz}$, which is highly challenging in OMC cavities [160].

- In comparison with OMC cavities, high-index disks can be excited from the farfield - though by using special arrangements of polarization [58], which eases the driving conditions. This way, we may think of OM metasurfaces formed by arrays of such disk so that their properties could be dynamically tuned at sub-nanosecond speeds, leading to ultrafast reconfigurable metasurfaces. In general, our BIC-modes OM cavities should play a role in applications benefiting from the formation of arrays, not operated in the resolved sideband regime, such as synchronization of mechanical resonators [51] or nonreciprocal phonon transport [59].

Finally, another significant feature involving most of the OMC in crystal cavities or high-index disks is the phonon leakage into the substrate. In order to avoid that, as we have seen, a released of the structures or the formation of thin pedestals in the substrate material is needed to ensure high performance. However, this imposes some limitations as the releasing process itself can degrade other components in the chip due to the use of HF baths and it turns the structures more fragile, leading them to even collapse. Because of that, unreleased proposals are needed to ensure good mechanical confinement thus enabling the light-sound interaction. In this thesis, we finally addressed this problem by studying a configuration where AO interaction has been broadly studied: Brillouin waveguides. In regards to that:

- We have shown a novel approach towards unreleased cavity and waveguide optomechanics in silicon technology. The presented structure relies on vertical engineering: 
a thick layer of silicon nitride separates the silicon core from the silica substrate, thus reducing the mechanical leakage into the substrate whilst enabling loss-less photonic guidance in the silicon core. Values of the Brillouin gain around 300 $1 / \mathrm{Wm}$ can be achieved in several configurations for mechanical modes with frequencies above $10 \mathrm{GHz}$ when a mechanical $\mathrm{Q}$ factor equal to $10^{3}$ is considered, which is usually the case in room-temperature optomechanics in silicon. Much higher values of the Brillouin gain are attainable assuming operation at low temperature because of the decrease of the acoustic material losses and subsequent increase of the mechanical $\mathrm{Q}$ factor.

- OM structures based on this multilayer system could be fabricated using standard silicon technology. Our finding could pave the way towards unreleased silicon optomechanical circuits, which would overcome the limitations arising from the need to release the silicon core in standard optomechanical circuitry. Moreover, the concept of vertical engineering could be extended to other high-index OM technologies as long as an adequate material for the intermediate layer is employed. 


\section{Appendices}

\section{Contents}

OM crystal cavities fabrication $\ldots \ldots \ldots \ldots \ldots 2$

B Taper loop fabrication $\ldots \ldots \ldots \ldots \ldots \ldots$

C OFDM signal description . . . . . . . . . . . 96

D Author's merits ................ 97

D.1 Publications . . . . . . . . . . . . . . . . . . 97

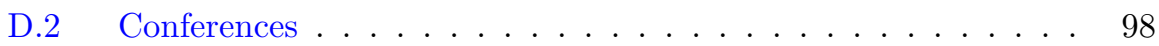




\section{A OM CRYSTAL CAVITIES FABRICATION}

The fabrication of all the samples presented along this thesis (Chapters 2 to 4) have been carried out at the Nanophotonics Technology Center in Valencia. A detailed scheme depicted the different steps of the fabrication procedure is shown in Fig. A1. (a) The structures were fabricated using a silicon-on-insulator wafer (SOITEC) with a monocrystalline silicon layer of $200 \mathrm{~nm}$ on top of a $2 \mu \mathrm{m}$ buried oxide layer, on top of a $\mathrm{Si}$ substrate. (b) Then, a polymethyl methacrylate (PMMA) is spin coated to perform as a positive resist layer of $170 \mathrm{~nm}$ of thickness and then baked at $950 \mathrm{~K}$. (c) Once the PMMA is evenly distributed over the sample, the patterned structure is written by electron beam lithography, performed with a Raith150 tool, at an acceleration voltage of $10 \mathrm{keV}$. (d) When the pattern have been exposed, the sample is developed with MIBK/IPA which removes the exposed areas. Therefore, the remaining resists acts as a mask and the pattern is transferred to the silicon layer by reactive ion etching using $S F_{6} / C_{4} F_{8}$ gases. (e) Finally, the silicon is etched, a buffered hydrofluoric (HF) acid is applied to remove the buried oxide layer to release the structured nanobeams.

(a)

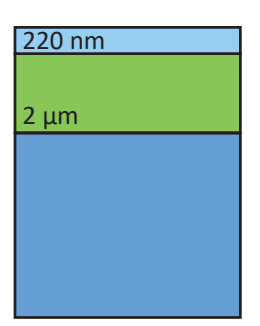

Mono-crystal Si

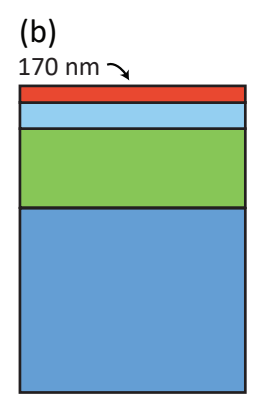

$\sqsubset$ Buried oxide layer

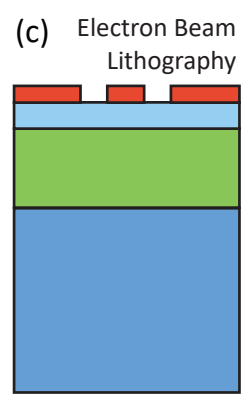

Si substrate

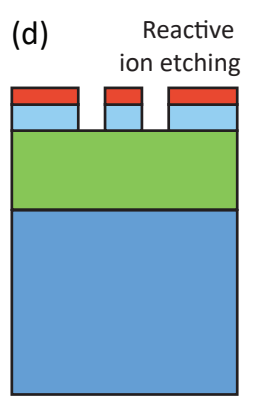

ए PMMA resist (e) HF etch

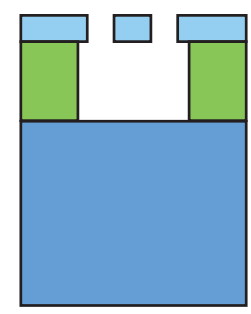

Figure A1: (a) Original SOITEC sample with 220 of nm mono-crystal Si on top of $2 \mu \mathrm{m}$ of buried oxide layer. (b) Positive PMMA resist spin-coating and baking. (c) Electron beam lithography exposure. (d) Reactive ion etching. (e) HF etch.

SEM images of a set of fabricated OM crystal cavities is presented in Fig. A2(a), where a close view can be appreciated in Fig. A2(b). From both of them it can be appreciated that the buried oxide layer under the top silicon patterned structure has been totally removed, thus resulting in suspended OMCs. 

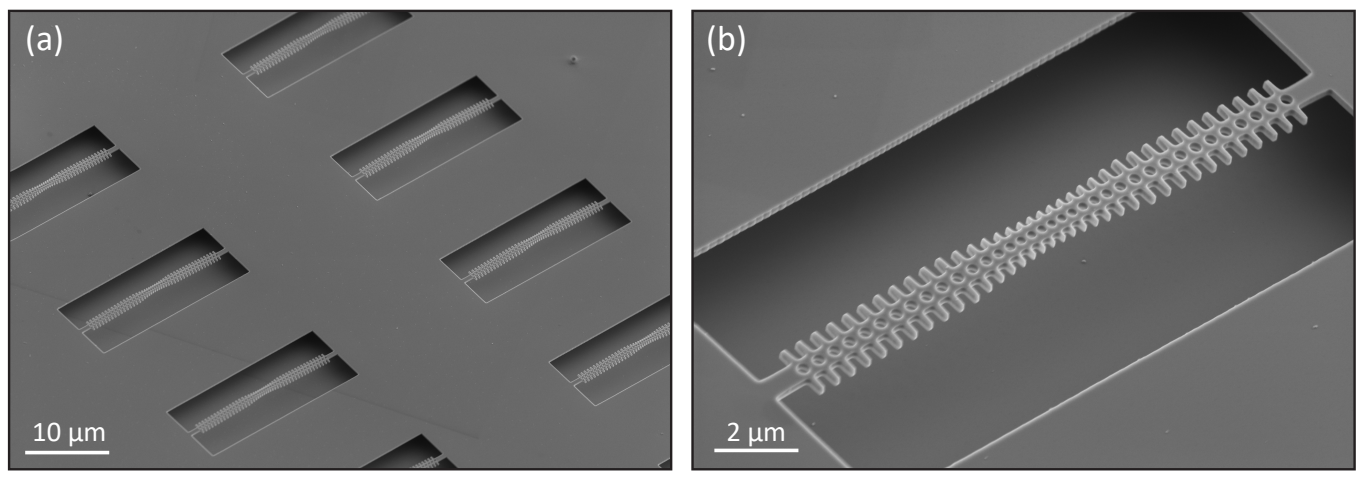

Figure A2: (a) Scanning microscopy image overview of a set patterned structures. (b) SEM close view of a released OM crystal cavity.

\section{B TAPER LOOP FABRICATION}

In pursuit of fabricate a tapered fiber loop to couple the optomechanical cavities in our experiments, we use the setup in Fig. B1 under room temperature and pressure conditions.

However, prior to the creation of the loop itself, a tapered fiber has to be fabricated. In order to do that, an input laser (New Focus Velocity TLB-6728) at a fixed wavelength is sent through a standard SMF-28 fiber, which is connected to a photodetector (Thorlabs PDA20CS-EC InGaAs Amplified Detector) to monitor all the fabrication process. Then, we remove an average of $3 \mathrm{~cm}$ of the external coating from a middle part of the fiber and place it on top of an hydrogen torch with two clamp holders, one at each side of the removed coated fiber. These clamp holders are placed on top of plates connected to motorized actuators, which will pull the fiber in the propagation direction depicted with red arrows in Fig. B1. This pulling process is performed when the removed coated fiber is heated by an hydrogen $\left(\mathrm{H}_{2}\right)$ flame.

The hydrogen flame is ignited externally with a burner, placed close to the hydrogen torch, when a stable hydrogen flow is present. This hydrogen flow is generated with the green circuit depicted in Fig. B1. Here, the green lines represent the $\mathrm{H}_{2}$ pipelines. The $\mathrm{H}_{2}$ up to $99.9995 \%$ of purity is generated with an hydrogen generator (QL-500 Hydrogen Generator). A gate valve (V1) located at the exit of the hydrogen generator is used to totally enable or disabled the hydrogen flow reaching the torch and the pressure is regulated via a flow regulating valve (V2 - MR3A13SVVT Brooks Mechanical Flowmeter). The generator operating output pressure is $0.2 \mathrm{MPa}$ and the final output flow is 200 $\mathrm{ml} / \mathrm{min}$.

Once we have those specifications of hydrogen flow and the flame is ignited, we start to pull the fiber with the motorized actuators. The pulling is performed at a constant velocity of $2.2 \mathrm{~mm} / \mathrm{s}$. During this process the transmitted signal is monitored with the photodetector, and the resulting signal is analyzed with an oscilloscope (Agilent Infiniium DSA80000B). The evolution of the transmitted signal in the overall process is shown in Fig. B2(a). A close view at different times is also presented in Fig. B2(b). Here, it can be seen an oscillation behavior corresponding to the transition from a monomode to a multimode fiber due to the the change in the fiber core diameter whilst it is being melted and pulled. Once the transmitted signal becomes stable again, corresponding to 


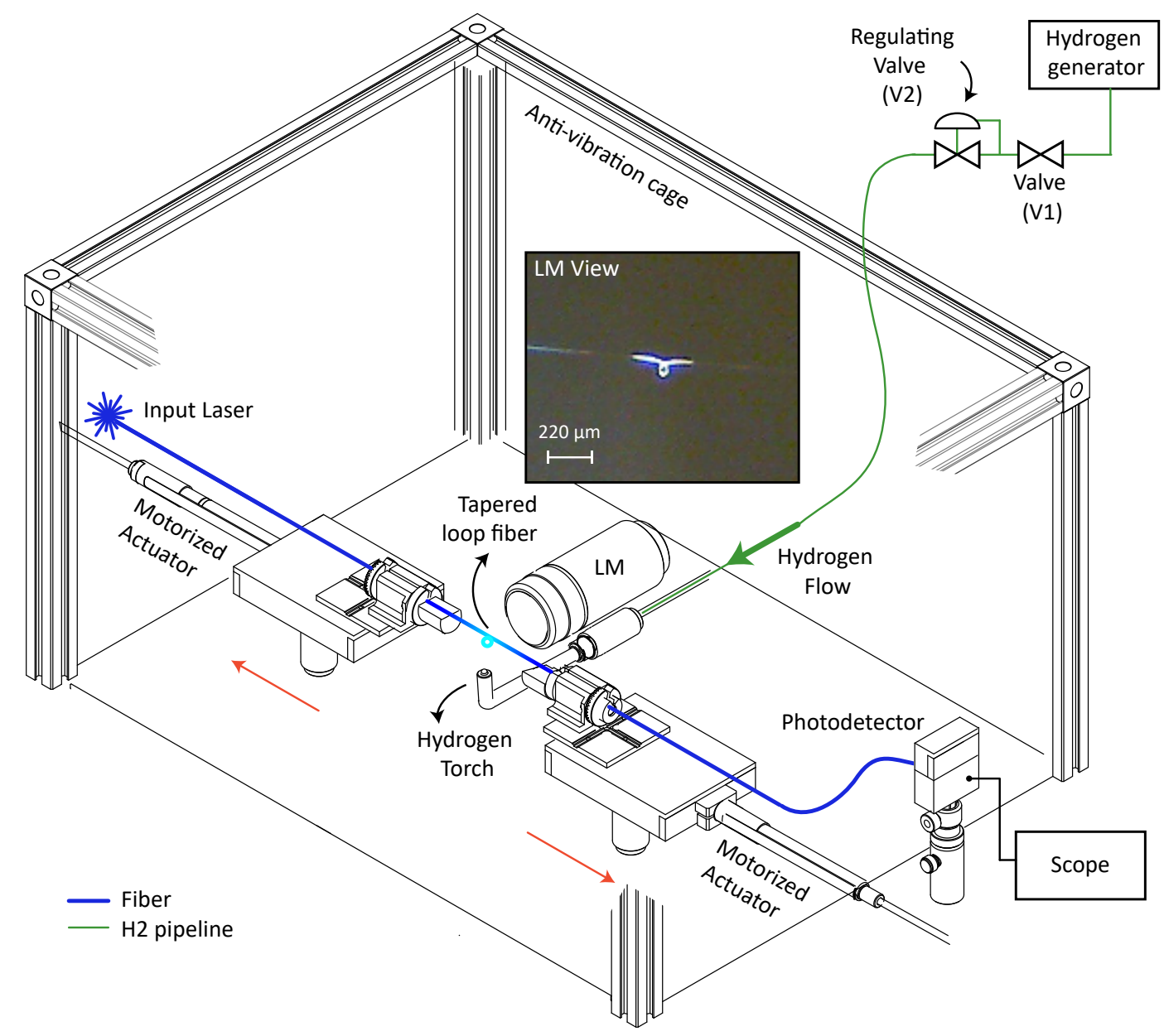

Figure B1: Tapered fiber loop fabrication setup. Light from a tunable laser at a fixed wavelength is sent through a SMF-28 fiber whilst it is heated with an hydrogen flame and pulled by two motorized actuators. The transmitted signal is measured with a photodetector for monitoring the overall process. The red arrows indicate the pulling sense. LM: Lateral Microscope. 
a monomode fiber condition, we stop simultaneously both the pulling and the hydrogen flame (with the valve V1), thus resulting in a monomode tapered fiber. The total pulling is around $18 \mathrm{~mm}$. It must be noted that a reduction of $10 \%-20 \%$ of the transmitted signal at the end of the process is experienced and some of the above specifications can slightly vary as a function of the environment humidity. The fabrication process can be also observed with a lateral microscope (LM) focused in the fiber.

(a)

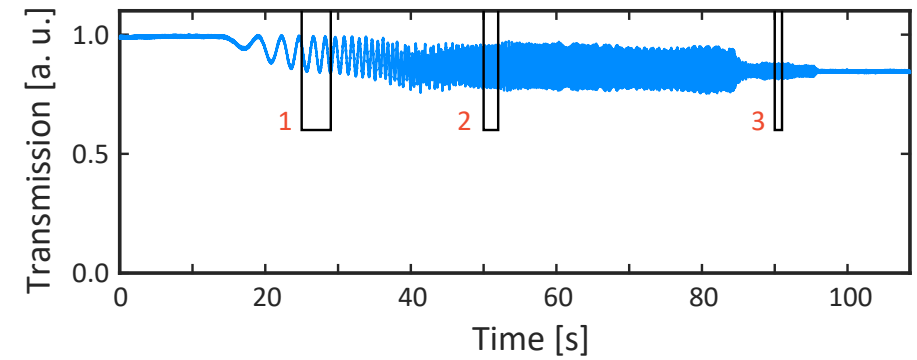

(b)

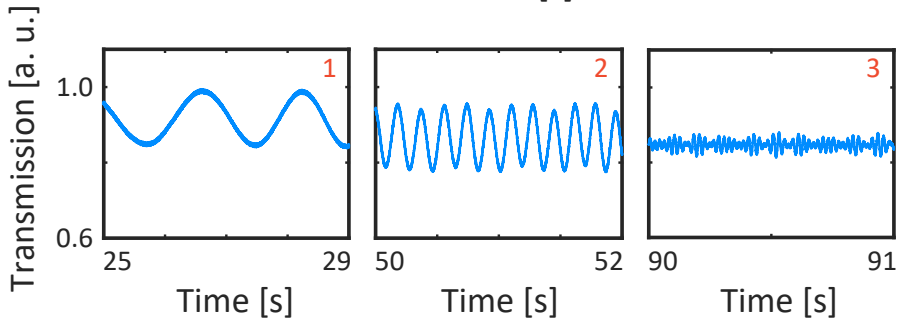

Figure B2: Evolution of the transmitted signal in the taper fiber fabrication.

Finally, to create the loop from the tapered fiber, one of the clamp holders is rotated. Next, the holders are moved closer to each other with the motorized actuators until the fiber loop appears. Once it has been created, the size of the loop can be tuned moving closer of further away both holders. An example of a fabricated tapered fiber loop image, taken with the laterial microscope, is presented in the inset of Fig. B1. 


\section{OFDM SIGNAL DESCRIPTION}

Concerning the OFDM signal used in Chapter 3, Table $\mathrm{C} 1$ summarizes the main parameters of the IEEE 802.16e WiMAX signal employed in our experiments. A downlink (DL) WiMAX signal following Partial Usage of Subchannels (PUSC) zone type is generated with a FFT of 1024, using 850 sub-carriers plus a DC carrier. The OFDM signal is generated with a sampling factor $n$ (also known $F_{s} / B W$ ) defined as allowed by the IEEE $802.16 \mathrm{e}$ standard, being $n=8 / 7$ for channel bandwidths multiple of $1.75 \mathrm{MHz}$. The Frame Control Header (FCH) and DL-MAP contains information about the frame content (location and modulation of the individual bursts) using BPSK-modulated pilots. The DL-PUSC data can be modulated with quadrature phase shift keying (QPSK), 16QAM or 64QAM providing up to $70 \mathrm{Mbps}$ per user in mobile scenarios with moving users up to $60 \mathrm{kph}$. The cyclic redundancy check (CRC) is calculated with the bits of each byte of the medium access control protocol data unit (MAC PDU) with the most significant bit (MSB) first. The pseudo-random binary sequence (PRBS) generation method follows Cor1/D3 for data randomization. The WiMAX signal after up- and downconversion is analyzed with a R\&S FSQ40 to demodulate the OFDM signal with $15 \mathrm{~ms}$ capture time and the same demodulation settings as described in Table C1.

Table C1: Main parameters of the OFDM signal following IEEE 802.16e WiMAX standard.

\begin{tabular}{cc|cc}
\hline Parameter & Value & Parameter & Value \\
\hline FFT size & 1024 & $N_{\text {used }}$ & 850 \\
Center frequency & $3.9 \mathrm{GHz}$ & Channel BW & $3.5,7,14$ or $28 \mathrm{MHz}$ \\
Guard period G & $1 / 4$ & Sampling factor $n$ & $8 / 7$ \\
MAC CRC order & MSB First & PRBS Method & Cor1/D3 \\
Zone type & DL-PUSC & Capture time & $15 \mathrm{~ms}$ \\
\hline
\end{tabular}




\section{AUTHOR'S MERITS}

\section{D.1 PUBLICATIONS}

- L. Mercadé and A. Martínez, "Optomechanical microwave oscillators," Submitted to Reviews of Electromagnetics, (2021)

- L. Mercadé, M. Morant, A. Griol, R. Llorente and A. Martínez, "Application of Ultracompact Silicon Optomechanical Cavities to the Frequency Conversion of Wireless Signals," Submitted to Optica, (2021)

- L. Mercadé, K. Pelka, R. Burgwal, A. Xuereb, A. Martínez and E. Verhagen, "Floquet phonon lasing in multimode optomechanical systems," Submitted to Physical Review Letters, (2021)

- L. Mercadé, A. V. Korovin, Y. Pennec, J. Ahopelto, B. Djafari Rouhani, and A. Martínez, "Vertical engineering for large Brillouin gain in unreleased silicon-based waveguides," Physical Review Applied, 15, 034021 (2021)

- L. Mercadé, A. Barreda and A. Martínez, "Dispersive optomechanics of supercavity modes in high-index disks," Optics Letters, 45, 5238-5241 (2020)

- L. Mercadé, L. L. Martín, A. Griol, D. Navarro-Urrios, and A. Martínez, "Microwave oscillator and frequency comb in a silicon optomechanical cavity with a full phononic bandgap," Nanophotonics, 9, 11, 3535-3544 (2020)

- A. Korsbäck, F. Djurabekova, L. Mercadé, I. Profatilova, E. Rodriguez Castro, W. Wuensch, S. Calatroni, and T. Ahlgren, "Vacuum electrical breakdown conditioning study in a parallel plate electrode pulsed dc system," Physical Review Accelerators and Beams, 23, 033102 (2020)

- D. Navarro-Urrios, N.E. Capuj, J. Maire, M. Colombano, J. Jaramillo-Fernandez, E. Chavez-Angel, L. L. Martin, L. Mercadé, A. Griol, A. Martínez, C. M. Sotomayor-Torres, and J. Ahopelto, "Nanocrystalline silicon optomechanical cavities," Optics Express, 26, 9829-9839 (2018)

- D. Bañón-Caballero, J. M. Socuéllanos, R. Mata Sanz, L. Mercadé, B. Gimeno, V. E. Boria, D. Raboso, V. E. Semenov, E. I. Rakova, J. F. Sánchez-Royo and A. Segura, "Study of the Secondary Electron Yield in Dielectrics Using Equivalent Circuital Models," IEEE Transactions on Plasma Science, 46, 4, 859-867 (2018)

- E. Bronchalo, A. Coves, R. Mata, B. Gimeno, I. Montero, L. Galán, V. E. Boria, L. Mercadé and E. Sanchís-Kilders, "Secondary Electron Emission of Pt: Experimental Study and Comparison With Models in the Multipactor Energy Range," IEEE Transactions on Electron Devices, 63, 8, 3270-3277 (2016) 


\section{D.2 CONFERENCES}

- L. Mercadé, L. L. Martín-Rodríguez, A. Griol, D. Navarro-Urrios and A. Martínez Abietar, "Optomechanical microwave oscillator and frequency comb generation in a full phononic bandgap 1D optomechanical crystal cavity," in European Optical Society Annual Meeting (EOSAM 2020) (Porto, Portugal, 2020)

- L. Mercadé, L. L. Martín-Rodríguez, A. Griol, D. Navarro-Urrios and A. Martínez Abietar, "Frequency comb and microwave generation with a full phononic bandgap 1D optomechanical crystal cavity," in 22nd European Conference on Integrated Optics (ECIO 2020) (Paris, France, 2020)

- C. Dumitrascu, M. Morant, L. Mercadé, T. Angelonova and R. Llorente, "PDMSbased Bragg diffraction grating glucose sensor integrable in a contact lens," in SPIE Photonics West 2020. Optical Fibers and Sensors for Medical Diagnostics and Treatment Applications XX (San Francisco, USA, 2020)

- L. Mercadé Morales, L. L. Martín-Rodríguez, A. Griol and A. Martínez Abietar, "Large coupling rate in optomechanical crystal cavities with a full phononic bandgap," in XXXVII Reunión Bienal de la Real Sociedad Española de Física (Zaragoza, Spain, 2019)

- L. Mercadé Morales, L. L. Martín-Rodríguez, A. Griol and A. Martínez Abietar, "Towards Large Coupling Rate in Optomechanical Crystal Cavities with a Full Phononic Bandgap," in 3rd Workshop on OptoMechanics and Brillouin Scattering (WOMBAT 2019) (Tel Aviv, Israel, 2019)

- L. Mercadé Morales, L. L. Martín-Rodríguez, A. Griol and A. Martínez Abietar, "Experimental optical and mechanical modes in a new one-dimensional optomechanical crystal cavity design," in International Workshop on Sound-enabled Nanotechnologies (IWSENT 2018) (Valencia, Spain, 2018)

- P. González-Santatecla, L. Mercadé Morales, L. L. Martín-Rodríguez and A. Martínez Abietar, "Optomechanical interaction in dielectric nanoparticles embedded in plasmonic nanoantenna gaps," in International Workshop on Sound-enabled Nanotechnologies (IWSENT 2018) (Valencia, Spain, 2018)

- L. Mercadé Morales and A. Martínez Abietar, "Nano-optomechanical crystal cavity design for totally confined gigahertz mechanical modes with a complete phononic bandgap," in X Reunión del Grupo Especializado de Física del Estado Sólido (GEFES 2018) (Valencia, Spain, 2018)

- L. Mercadé Morales and A. Martínez Abietar, "Totally confined gigahertz mechanical mode with a complete phononic bandgap for a nano-optomechanic cavity," in Nanophotonics and Micro/Nano Optics International Conference (NANOP 2017) (Barcelona, Spain, 2017)

- L. Mercadé Morales and A. Martínez Abietar, "Optomechanical cavity design with a complete phononic bandgap for a confined $5 \mathrm{GHz}$ acoustic mode," in II Workshop on OptoMechanics and Brillouin Scattering (WOMBAT 2017) (Besançon, France, 2017) 
- R. Mata Sanz, L. Mercadé Morales, D. Bañón, J. M. Socuellamos, B. Gimeno, V. Boria and D. Raboso, "Study of the charge processes involved in the secondary electron emission yield measurements of dielectric materials," in 9th International Workshop on Multipactor, Corona and Passive Intermodulation (Noordwijk, Netherlands, 2017)

- I. Profatilova, N. Pienimäki, X. Stragier, E. Rodriguez Castro, T. Lucas, L. Mercadé Morales, A. Korsback, S. Calatroni and W. Wuensch, "News from Pulsed DC System at CERN" in 6th International Workshop on Mechanisms of Vacuum Arcs (Jerusalem, Israel, 2017)

- I. Profatilova, N. Pienimäki, X. Stragier, E. Rodriguez Castro, L. Mercadé Morales, A. Korsback, S. Calatroni and W. Wuensch, "Recent results from the Pulsed DC systems," in CLIC Workshop 2017 (Geneva, Switzerland, 2017) 


\section{Bibliography}

[1] J. Kepler. De Cometis Libelli Tres (1619)

[2] M. Aspelmeyer, T. J. Kippenberg, and F. Marquardt. Cavity optomechanics. Rev. Mod. Phys. 86, 1391-1452 (2014)

[3] V. B. Braginskiı̌ and Y. I. Vorontsov. Quantum-mechanical limitations in macroscopic experiments and modern experimental technique. Soviet Physics Uspekhi 17(5), 644-650 (1975)

[4] A. Ashkin. Trapping of atoms by resonance radiation pressure. Phys. Rev. Lett. 40, 729-732 (1978)

[5] C. Fabre, M. Pinard, S. Bourzeix, A. Heidmann, E. Giacobino, and S. Reynaud. Quantum-noise reduction using a cavity with a movable mirror. Phys. Rev. A 49, 1337-1343 (1994)

[6] S. Mancini and P. Tombesi. Quantum noise reduction by radiation pressure. Phys. Rev. A 49, 4055-4065 (1994)

[7] H. J. Metcalf and P. van der Straten. Laser Cooling and Trapping (Springer, New York, 1999)

[8] G. Hechenblaikner, M. Gangl, P. Horak, and H. Ritsch. Cooling an atom in a weakly driven high-Q cavity. Phys. Rev. A 58, 3030-3042 (1998)

[9] V. Vuletić and S. Chu. Laser cooling of atoms, ions, or molecules by coherent scattering. Phys. Rev. Lett. 84, 3787-3790 (2000)

[10] T. J. Kippenberg and K. J. Vahala. Cavity optomechanics: Back-action at the mesoscale. Science 321(5893), 1172-1176 (2008)

[11] J. Chan, T. P. M. Alegre, A. H. Safavi-Naeini, J. T. Hill, A. Krause, S. Gröblacher, M. Aspelmeyer, and O. Painter. Laser cooling of a nanomechanical oscillator into its quantum ground state. Nature 478(7367), 89-92 (2011)

[12] A. H. Safavi-Naeini, S. Gröblacher, J. T. Hill, J. Chan, M. Aspelmeyer, and O. Painter. Squeezed light from a silicon micromechanical resonator. Nature 500(7461), 185-189 (2013)

[13] T. P. Purdy, P.-L. Yu, R. W. Peterson, N. S. Kampel, and C. A. Regal. Strong optomechanical squeezing of light. Phys. Rev. X 3, 031012 (2013) 
[14] T. A. Palomaki, J. D. Teufel, R. W. Simmonds, and K. W. Lehnert. Entangling mechanical motion with microwave fields. Science 342(6159), 710 (2013)

[15] I. S. Grudinin, A. B. Matsko, and L. Maleki. Brillouin lasing with a $\mathrm{CaF}_{2}$ whispering gallery mode resonator. Phys. Rev. Lett. 102, 043902 (2009)

[16] I. S. Grudinin, H. Lee, O. Painter, and K. J. Vahala. Phonon laser action in a tunable two-level system. Phys. Rev. Lett. 104, 083901 (2010)

[17] S. Weis, R. Rivière, S. Deléglise, E. Gavartin, O. Arcizet, A. Schliesser, and T. J. Kippenberg. Optomechanically induced transparency. Science 330(6010), 1520$1523(2010)$

[18] A. H. Safavi-Naeini, T. P. M. Alegre, J. Chan, M. Eichenfield, M. Winger, Q. Lin, J. T. Hill, D. E. Chang, and O. Painter. Electromagnetically induced transparency and slow light with optomechanics. Nature 472(7341), 69-73 (2011)

[19] F. Ruesink, M.-A. Miri, A. Alù, and E. Verhagen. Nonreciprocity and magneticfree isolation based on optomechanical interactions. Nature Communications $\mathbf{7}(1)$, $13662(2016)$

[20] E. Gil-Santos, J. J. Ruz, O. Malvar, I. Favero, A. Lemaître, P. M. Kosaka, S. García-López, M. Calleja, and J. Tamayo. Optomechanical detection of vibration modes of a single bacterium. Nature Nanotechnology 15(6), 469-474 (2020)

[21] J. Tamayo, P. M. Kosaka, J. J. Ruz, 1. San Paulo, and M. Calleja. Biosensors based on nanomechanical systems. Chem. Soc. Rev. 42(3), 1287-1311 (2013)

[22] T. Carmon, H. Rokhsari, L. Yang, T. J. Kippenberg, and K. J. Vahala. Temporal behavior of radiation-pressure-induced vibrations of an optical microcavity phonon mode. Phys. Rev. Lett. 94, 223902 (2005)

[23] T. J. Kippenberg, H. Rokhsari, T. Carmon, A. Scherer, and K. J. Vahala. Analysis of radiation-pressure induced mechanical oscillation of an optical microcavity. Phys. Rev. Lett. 95, 033901 (2005)

[24] A. Schliesser, P. Del'Haye, N. Nooshi, K. J. Vahala, and T. J. Kippenberg. Radiation pressure cooling of a micromechanical oscillator using dynamical backaction. Phys. Rev. Lett. 97, 243905 (2006)

[25] J. D. Thompson, B. M. Zwickl, A. M. Jayich, F. Marquardt, S. M. Girvin, and J. G. E. Harris. Strong dispersive coupling of a high-finesse cavity to a micromechanical membrane. Nature 452(7183), 72-75 (2008)

[26] R. Ma, A. Schliesser, P. Del'Haye, A. Dabirian, G. Anetsberger, and T. J. Kippenberg. Radiation-pressure-driven vibrational modes in ultrahigh-Q silica microspheres. Opt. Lett. 32(15), 2200-2202 (2007)

[27] M. Tomes and T. Carmon. Photonic micro-electromechanical systems vibrating at X-band (11-Ghz) rates. Phys. Rev. Lett. 102, 113601 (2009)

[28] X. Jiang, Q. Lin, J. Rosenberg, K. Vahala, and O. Painter. High-Q double-disk microcavities for cavity optomechanics. Opt. Express 17(23), 20911-20919 (2009) 
[29] M. Eichenfield, J. Chan, R. M. Camacho, K. J. Vahala, and O. Painter. Optomechanical crystals. Nature 462(7269), 78-82 (2009)

[30] J. Chan, A. H. Safavi-Naeini, J. T. Hill, S. Meenehan, and O. Painter. Optimized optomechanical crystal cavity with acoustic radiation shield. Applied Physics Letters 101(8), 081115 (2012)

[31] J. Gomis-Bresco, D. Navarro-Urrios, M. Oudich, S. El-Jallal, A. Griol, D. Puerto, E. Chavez, Y. Pennec, B. Djafari-Rouhani, F. Alzina, A. Martínez, and C. M. S. Torres. A one-dimensional optomechanical crystal with a complete phononic band gap. Nature Communications 5(1), 4452 (2014)

[32] J. D. Thompson, B. M. Zwickl, A. M. Jayich, F. Marquardt, S. M. Girvin, and J. G. E. Harris. Strong dispersive coupling of a high-finesse cavity to a micromechanical membrane. Nature 452(7183), 72-75 (2008)

[33] D. Kleckner, B. Pepper, E. Jeffrey, P. Sonin, S. M. Thon, and D. Bouwmeester. Optomechanical trampoline resonators. Opt. Express 19(20), 19708-19716 (2011)

[34] A. J. Maker and A. M. Armani. Fabrication of silica ultra high quality factor microresonators. Journal of visualized experiments 65(4164) (2012)

[35] X. Sun, K. Y. Fong, C. Xiong, W. H. P. Pernice, and H. X. Tang. Ghz optomechanical resonators with high mechanical q factor in air. Opt. Express 19(22), 22316-22321 (2011)

[36] A. Schliesser, R. Rivière, G. Anetsberger, O. Arcizet, and T. J. Kippenberg. Resolved-sideband cooling of a micromechanical oscillator. Nature Physics 4(5), 415-419 (2008)

[37] I. Favero, C. Metzger, S. Camerer, D. König, H. Lorenz, J. P. Kotthaus, and K. Karrai. Optical cooling of a micromirror of wavelength size. Appl. Phys. Lett. 90(10), 104101 (2007)

[38] A. H. Safavi-Naeini, T. P. M. Alegre, M. Winger, and O. Painter. Optomechanics in an ultrahigh-Q two-dimensional photonic crystal cavity. Appl. Phys. Lett. 97(18), 181106 (2010)

[39] J. Capmany and D. Novak. Microwave photonics combines two worlds. Nature Photonics 1(6), 319-330 (2007)

[40] Z. Tang, Y. Li, J. Yao, and S. Pan. Photonics-based microwave frequency mixing: Methodology and applications. Laser $\& 3$ Photonics Reviews 14(1), 1800350 (2020)

[41] M. Hossein-Zadeh and K. J. Vahala. Photonic RF down-converter based on optomechanical oscillation. IEEE Photonics Technology Letters 20(4), 234-236 (2008)

[42] M. Hossein-Zadeh and K. J. Vahala. An optomechanical oscillator on a silicon chip. IEEE Journal of Selected Topics in Quantum Electronics 16(1), 276-287 (2010) 
[43] J. G. Huang, H. Cai, Y. D. Gu, L. K. Chin, J. H. Wu, T. N. Chen, Z. C. Yang, Y. L. Hao, and A. Q. Liu. Torsional frequency mixing and sensing in optomechanical resonators. Applied Physics Letters 111(11), 111102 (2017)

[44] I. Ghorbel, F. Swiadek, R. Zhu, D. Dolfi, G. Lehoucq, A. Martin, G. Moille, L. Morvan, R. Braive, S. Combrié, and A. De Rossi. Optomechanical gigahertz oscillator made of a two photon absorption free piezoelectric iii/v semiconductor. APL Photonics 4(11), 116103 (2019)

[45] IEEE 802.16e Standard for Local and Metropolitan Area Networks - Part 16: Air Interface for Fixed and Mobile Broadband Wireless Access Systems - Amendment for Physical and Medium Access Control Layers for Combined Fixed and Mobile Operation in Licensed Bands (2006)

[46] Qualcomm. Global update on spectrum for 4G \& 5G. Dec. 2020 (available at https://www.qualcomm.com/media/documents/files/spectrum-for$4 \mathrm{~g}$-and-5g.pdf)

[47] G. Heinrich, M. Ludwig, J. Qian, B. Kubala, and F. Marquardt. Collective dynamics in optomechanical arrays. Phys. Rev. Lett. 107, 043603 (2011)

[48] M. Zhang, G. S. Wiederhecker, S. Manipatruni, A. Barnard, P. McEuen, and M. Lipson. Synchronization of micromechanical oscillators using light. Phys. Rev. Lett. 109, 233906 (2012)

[49] C. A. Holmes, C. P. Meaney, and G. J. Milburn. Synchronization of many nanomechanical resonators coupled via a common cavity field. Phys. Rev. E 85, 066203 (2012)

[50] N. Lörch, S. E. Nigg, A. Nunnenkamp, R. P. Tiwari, and C. Bruder. Quantum synchronization blockade: Energy quantization hinders synchronization of identical oscillators. Phys. Rev. Lett. 118, 243602 (2017)

[51] M. F. Colombano, G. Arregui, N. E. Capuj, A. Pitanti, J. Maire, A. Griol, B. Garrido, A. Martinez, C. M. Sotomayor-Torres, and D. Navarro-Urrios. Synchronization of optomechanical nanobeams by mechanical interaction. Phys. Rev. Lett. 123, $017402(2019)$

[52] K. Pelka, V. Peano, and A. Xuereb. Chimera states in small optomechanical arrays. Phys. Rev. Research 2, 013201 (2020)

[53] G. Madiot, F. Correia, S. Barbay, and R. Braive. Bichromatic synchronized chaos in coupled optomechanical nanoresonators (2020), 2005.08896

[54] M. Zhang, S. Shah, J. Cardenas, and M. Lipson. Synchronization and phase noise reduction in micromechanical oscillator arrays coupled through light. Phys. Rev. Lett. 115, 163902 (2015)

[55] S. Walter and F. Marquardt. Classical dynamical gauge fields in optomechanics. New Journal of Physics 18(11), 113029 (2016)

[56] I. Mahboob, H. Okamoto, and H. Yamaguchi. An electromechanical ising hamiltonian. Sci Adv 2(6), e1600236 (2016) 
[57] M. V. Rybin, K. L. Koshelev, Z. F. Sadrieva, K. B. Samusev, A. A. Bogdanov, M. F. Limonov, and Y. S. Kivshar. High-Q supercavity modes in subwavelength dielectric resonators. Phys. Rev. Lett. 119, 243901 (2017)

[58] K. Koshelev, S. Kruk, E. Melik-Gaykazyan, J.-H. Choi, A. Bogdanov, H.-G. Park, and Y. Kivshar. Subwavelength dielectric resonators for nonlinear nanophotonics. Science 367(6475), 288-292 (2020)

[59] J. P. Mathew, J. d. Pino, and E. Verhagen. Synthetic gauge fields for phonon transport in a nano-optomechanical system. Nature Nanotechnology 15(3), 198$202(2020)$

[60] E. A. Kittlaus, H. Shin, and P. T. Rakich. Large brillouin amplification in silicon. Nature Photonics 10(7), 463-467 (2016)

[61] B. J. Eggleton, C. G. Poulton, P. T. Rakich, M. J. Steel, and G. Bahl. Brillouin integrated photonics. Nature Photonics 13(10), 664-677 (2019)

[62] R. Van Laer, R. Baets, and D. Van Thourhout. Unifying Brillouin scattering and cavity optomechanics. Phys. Rev. A 93, 053828 (2016)

[63] R. Van Laer. Light-sound interaction in nanoscale silicon waveguides. Doctoral Thesis, Ghent University (2016)

[64] J. Chan. Laser Cooling of an Optomechanical Crystal Resonator to Its Quantum Ground State of Motion. Doctoral Thesis, California Institute of Technology (2012)

[65] M. L. Gorodetksy, A. Schliesser, G. Anetsberger, S. Deleglise, and T. J. Kippenberg. Determination of the vacuum optomechanical coupling rate using frequency noise calibration. Opt. Express 18(22), 23236-23246 (2010)

[66] J. D. Teufel, T. Donner, D. Li, J. W. Harlow, M. S. Allman, K. Cicak, A. J. Sirois, J. D. Whittaker, K. W. Lehnert, and R. W. Simmonds. Sideband cooling of micromechanical motion to the quantum ground state. Nature 475(7356), 359-363 (2011)

[67] D. Navarro-Urrios, J. Gomis-Bresco, S. El-Jallal, M. Oudich, A. Pitanti, N. Capuj, A. Tredicucci, F. Alzina, A. Griol, Y. Pennec, B. Djafari-Rouhani, A. Martínez, and C. M. Sotomayor Torres. Dynamical back-action at $5.5 \mathrm{ghz}$ in a corrugated optomechanical beam. AIP Advances 4(12), 124601 (2014)

[68] K. Schneider, Y. Baumgartner, S. Hönl, P. Welter, H. Hahn, D. J. Wilson, L. Czornomaz, and P. Seidler. Optomechanics with one-dimensional gallium phosphide photonic crystal cavities. Optica 6(5), 577-584 (2019)

[69] G. P. Agrawal. Nonlinear fiber optics. № Accessed from https://nla.gov.au/nla.cat-vn610751 in Quantum electronics-principles and applications. (Academic Press, Boston, 1989)

[70] L. Brillouin. Diffusion de la lumière et des rayons X par un corps transparent homogène. Ann. Phys. 9(17), 88 - 122 (1922) 
[71] R. Y. Chiao, C. H. Townes, and B. P. Stoicheff. Stimulated Brillouin scattering and coherent generation of intense hypersonic waves. Phys. Rev. Lett. 12, 592-595 (1964)

[72] R. H. Stolen. The early years of fiber nonlinear optics. J. Lightwave Technol. 26(9), 1021-1031 (2008)

[73] B. Morrison. Stimulated Brillouin Scattering in Integrated Circuits: Platforms and Applications. Doctoral Thesis, School of Physics (2017)

[74] J.-C. Beugnot, S. Lebrun, G. Pauliat, H. Maillotte, V. Laude, and T. Sylvestre. Brillouin light scattering from surface acoustic waves in a subwavelength-diameter optical fibre. Nature Communications 5(1), 5242 (2014)

[75] R. Pant, C. G. Poulton, D.-Y. Choi, H. Mcfarlane, S. Hile, E. Li, L. Thevenaz, B. Luther-Davies, S. J. Madden, and B. J. Eggleton. On-chip stimulated Brillouin scattering. Opt. Express 19(9), 8285-8290 (2011)

[76] M. Merklein, I. V. Kabakova, T. F. S. Büttner, D.-Y. Choi, B. Luther-Davies, S. J. Madden, and B. J. Eggleton. Enhancing and inhibiting stimulated brillouin scattering in photonic integrated circuits. Nature Communications 6(1), 6396 (2015)

[77] P. T. Rakich, C. Reinke, R. Camacho, P. Davids, and Z. Wang. Giant enhancement of stimulated brillouin scattering in the subwavelength limit. Phys. Rev. X 2, $011008(2012)$

[78] V. Laude and J.-C. Beugnot. Lagrangian description of brillouin scattering and electrostriction in nanoscale optical waveguides. New Journal of Physics 17(12), $125003(2015)$

[79] H. Shin, W. Qiu, R. Jarecki, J. A. Cox, R. H. Olsson, A. Starbuck, Z. Wang, and P. T. Rakich. Tailorable stimulated Brillouin scattering in nanoscale silicon waveguides. Nature Communications 4(1), 1944 (2013)

[80] R. W. Boyd. Nonlinear Optics, Third Edition. 3rd edón . (Academic Press, Inc., USA, 2008)

[81] W. Qiu, P. T. Rakich, H. Shin, H. Dong, M. Soljačić, and Z. Wang. Stimulated Brillouin scattering in nanoscale silicon step-index waveguides: a general framework of selection rules and calculating sbs gain. Opt. Express 21(25), 31402-31419 (2013)

[82] A. H. Safavi-Naeini, D. Van Thourhout, R. Baets, and R. Van Laer. Controlling phonons and photons at the wavelength scale: integrated photonics meets integrated phononics. Optica 6(2), 213-232 (2019)

[83] M.-A. Miri, G. D'Aguanno, and A. Alù. Optomechanical frequency combs. New Journal of Physics 20(4), 043013 (2018)

[84] V. Torres-Company and A. M. Weiner. Optical frequency comb technology for ultra-broadband radio-frequency photonics. Laser $\&$ Photonics Reviews 8(3), 368393 (2014) 
[85] Y. Pennec, B. D. Rouhani, C. Li, J. M. Escalante, A. Martinez, S. Benchabane, V. Laude, and N. Papanikolaou. Band gaps and cavity modes in dual phononic and photonic strip waveguides. AIP Advances 1(4), 041901 (2011)

[86] A. G. Krause, J. T. Hill, M. Ludwig, A. H. Safavi-Naeini, J. Chan, F. Marquardt, and O. Painter. Nonlinear radiation pressure dynamics in an optomechanical crystal. Phys. Rev. Lett. 115, 233601 (2015)

[87] L. Qiu, I. Shomroni, P. Seidler, and T. J. Kippenberg. High-fidelity laser cooling to the quantum ground state of a silicon nanomechanical oscillator (2019), 1903. 10242

[88] K. Fang, M. H. Matheny, X. Luan, and O. Painter. Optical transduction and routing of microwave phonons in cavity-optomechanical circuits. Nature Photonics 10, 489 (2016)

[89] G. S. MacCabe, H. Ren, J. Luo, J. D. Cohen, H. Zhou, A. Sipahigil, M. Mirhosseini, and O. Painter. Phononic bandgap nano-acoustic cavity with ultralong phonon lifetime (2019), 1901.04129

[90] D. Navarro-Urrios, N. E. Capuj, J. Gomis-Bresco, F. Alzina, A. Pitanti, A. Griol, A. Martínez, and C. M. Sotomayor Torres. A self-stabilized coherent phonon source driven by optical forces. Scientific Reports 5, 15733 (2015)

[91] D. Navarro-Urrios, N. E. Capuj, M. F. Colombano, P. D. García, M. Sledzinska, F. Alzina, A. Griol, A. Martínez, and C. M. Sotomayor-Torres. Nonlinear dynamics and chaos in an optomechanical beam. Nature Communications 8(1), 14965 (2017)

[92] M. Oudich, S. El-Jallal, Y. Pennec, B. Djafari-Rouhani, J. Gomis-Bresco, D. Navarro-Urrios, C. M. Sotomayor Torres, A. Martínez, and A. Makhoute. Optomechanic interaction in a corrugated phoxonic nanobeam cavity. Phys. Rev. B 89, $245122(2014)$

[93] J. D. Joannopoulos, S. G. Johnson, J. N. Winn, and R. D. Meade. Photonic Crystals: Molding the Flow of Light (Princeton University Press, 2008)

[94] RSoft Bandsolve. URL https://www.synopsys.com/photonic-solutions/ rsoft-photonic-device-tools/passive-device-bandsolve.html

[95] V. Laude and J.-C. Beugnot. Generation of phonons from electrostriction in smallcore optical waveguides. AIP Advances 3(4), 042109 (2013)

[96] C. Charles, B. Bonello, and F. Ganot. Propagation of guided elastic waves in 2D phononic crystals. Ultrasonics 44, e1209 - e1213. Proceedings of Ultrasonics International (UI'05) and World Congress on Ultrasonics (WCU) (2006)

[97] COMSOL Multiphysics 5.3a

[98] Y. Pennec, V. Laude, N. Papanikolaou, B. Djafari-Rouhani, M. Oudich, S. E. Jallal, J. C. Beugnot, J. M. Escalante, and A. Martínez. Modeling light-sound interaction in nanoscale cavities and waveguides. Nanophotonics 3(6), $413-440$ (2014) 
[99] S. G. Johnson, M. Ibanescu, M. A. Skorobogatiy, O. Weisberg, J. D. Joannopoulos, and Y. Fink. Perturbation theory for maxwell's equations with shifting material boundaries. Phys. Rev. E 65, 066611 (2002)

[100] E. Gavartin, R. Braive, I. Sagnes, O. Arcizet, A. Beveratos, T. J. Kippenberg, and I. Robert-Philip. Optomechanical coupling in a two-dimensional photonic crystal defect cavity. Phys. Rev. Lett. 106, 203902 (2011)

[101] L. Mercadé, L. L. Martín, A. Griol, D. Navarro-Urrios, and A. Martínez. Microwave oscillator and frequency comb in a silicon optomechanical cavity with a full phononic bandgap. Nanophotonics 9(11), 3535-3544 (2020)

[102] R. Leijssen. Measuring mechanical motion using light confined at the nanoscale. Doctoral Thesis, Eindhoven University of Technology (2017)

[103] M. Eichenfield, R. Camacho, J. Chan, K. J. Vahala, and O. Painter. A picogramand nanometre-scale photonic-crystal optomechanical cavity. Nature 459(7246), $550-555(2009)$

[104] K. E. Grutter, M. I. Davanço, and K. Srinivasan. Slot-mode optomechanical crystals: a versatile platform for multimode optomechanics. Optica 2(11), 994-1001 (2015)

[105] L. Maleki. The optoelectronic oscillator. Nature Photonics 5(12), 728-730 (2011)

[106] X. S. Yao and L. Maleki. Optoelectronic microwave oscillator. J. Opt. Soc. Am. $B$ 13(8), 1725-1735 (1996)

[107] J. Li, H. Lee, and K. J. Vahala. Microwave synthesizer using an on-chip Brillouin oscillator. Nature Communications 4(1), 2097 (2013)

[108] L. Neumeier. Novel regimes of quantum optomechanics. Doctoral Thesis, ICFOThe Institute of Photonic Sciences, Universtitat Politècnica de Catalunya (2018)

[109] F. Pan, K. Cui, G. Bai, X. Feng, F. Liu, W. Zhang, and Y. Huang. Radiationpressure-antidamping enhanced optomechanical spring sensing. ACS Photonics 5(10), 4164-4169 (2018)

[110] S. Tallur, S. Sridaran, S. A. Bhave, and T. Carmon. Phase noise modeling of optomechanical oscillators. In 2010 IEEE International Frequency Control Symposium, pages. 268-272 (2010)

[111] S. Tallur, S. Sridaran, and S. A. Bhave. A monolithic radiation-pressure driven, low phase noise silicon nitride opto-mechanical oscillator. Opt. Express 19(24), 24522-24529 (2011)

[112] E. Rubiola. Phase Noise and Frequency Stability in Oscillators. The Cambridge RF and Microwave Engineering Series (Cambridge University Press, 2008)

[113] https://www.keysight.com/us/en/assets/7018-08250/data-sheets/5989-7572.pdf

[114] S. Sridaran and S. A. Bhave. 1.12Ghz opto-acoustic oscillator. In 2012 IEEE 25th International Conference on Micro Electro Mechanical Systems (MEMS), pages. 664-667 (2012) 
[115] C. Mathai, S. A. Bhave, and S. Tallur. Modeling the colors of phase noise in optomechanical oscillators. OSA Continuum 2(7), 2253-2259 (2019)

[116] J. Tang, T. Hao, W. Li, D. Domenech, R. B. nos, P. M. noz, N. Zhu, J. Capmany, and M. Li. Integrated optoelectronic oscillator. Opt. Express 26(9), 12257-12265 (2018)

[117] X. Luan, Y. Huang, Y. Li, J. F. McMillan, J. Zheng, S.-W. Huang, P.-C. Hsieh, T. Gu, D. Wang, A. Hati, D. A. Howe, G. Wen, M. Yu, G. Lo, D.-L. Kwong, and C. W. Wong. An integrated low phase noise radiation-pressure-driven optomechanical oscillator chipset. Scientific Reports 4, 6842 (2014)

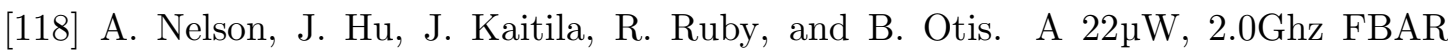
oscillator. In 2011 IEEE Radio Frequency Integrated Circuits Symposium, pages. $1-4(2011)$

[119] T. Notes. Jitter and phase noise - the fundamentals. Inf. téc., SEIKO EPSON CORPORATION

[120] J. Zheng, Y. Li, N. Goldberg, M. McDonald, X. Luan, A. Hati, M. Lu, S. Strauf, T. Zelevinsky, D. A. Howe, and C. Wei Wong. Feedback and harmonic locking of slot-type optomechanical oscillators to external low-noise reference clocks. Applied Physics Letters 102(14), 141117 (2013)

[121] M. S. Kang, A. Nazarkin, A. Brenn, and P. S. J. Russell. Tightly trapped acoustic phonons in photonic crystal fibres as highly nonlinear artificial raman oscillators. Nature Physics 5(4), 276-280 (2009)

[122] P. Del'Haye, A. Schliesser, O. Arcizet, T. Wilken, R. Holzwarth, and T. J. Kippenberg. Optical frequency comb generation from a monolithic microresonator. Nature 450(7173), 1214-1217 (2007)

[123] T. J. Kippenberg, R. Holzwarth, and S. A. Diddams. Microresonator-based optical frequency combs. Science 332(6029), 555-559 (2011)

[124] A. A. Savchenkov, A. B. Matsko, V. S. Ilchenko, D. Seidel, and L. Maleki. Surface acoustic wave opto-mechanical oscillator and frequency comb generator. Opt. Lett. 36(17), 3338-3340 (2011)

[125] D. Navarro-Urrios, J. Gomis-Bresco, N. E. Capuj, F. Alzina, A. Griol, D. Puerto, A. Martínez, and C. M. Sotomayor-Torres. Optical and mechanical mode tuning in an optomechanical crystal with light-induced thermal effects. Journal of Applied Physics 116(9), 093506 (2014)

[126] D. Marpaung, J. Yao, and J. Capmany. Integrated microwave photonics. Nature Photonics 13(2), 80-90 (2019)

[127] WiMAX FORUM. URL https://wimaxforum.org/

[128] D. F. Ramian. Time domain oscillator stability measurement allan variance application note. Inf. téc., Rohde \& Schwarz (2015) 
[129] E. Gavartin, P. Verlot, and T. J. Kippenberg. Stabilization of a linear nanomechanical oscillator to its thermodynamic limit. Nature Communications 4(1), 2860 (2013)

[130] W. Yu, W. C. Jiang, Q. Lin, and T. Lu. Cavity optomechanical spring sensing of single molecules. Nature Communications 7(1), 12311 (2016)

[131] A. C. L. C. Ayan Sengupta, Alberto Rico-Alvarino. Cellular terrestrial broadcast-physical layer evolution from $3 \mathrm{GPP}$ release 9 to release 16. IEEE Transactions on Broadcasting 66(2), 459-470 (2020)

[132] U. Kemiktarak, M. Durand, M. Metcalfe, and J. Lawall. Mode competition and anomalous cooling in a multimode phonon laser. Phys. Rev. Lett. 113, 030802 (2014)

[133] W. E. Lamb. Theory of an optical maser. Phys. Rev. 134, A1429-A1450 (1964)

[134] G. J. Hall, M. Ebrahimzadeh, A. Robertson, G. P. A. Malcolm, and A. I. Ferguson. Synchronously pumped optical parametric oscillators using all-solid-state pump lasers. J. Opt. Soc. Am. B 10(11), 2168-2179 (1993)

[135] C. Metzger, M. Ludwig, C. Neuenhahn, A. Ortlieb, I. Favero, K. Karrai, and F. Marquardt. Self-induced oscillations in an optomechanical system driven by bolometric backaction. Phys. Rev. Lett. 101, 133903 (2008)

[136] M. J. Weaver, F. Buters, F. Luna, H. Eerkens, K. Heeck, S. de Man, and D. Bouwmeester. Coherent optomechanical state transfer between disparate mechanical resonators. Nature Communications 8(1), 824 (2017)

[137] H. Xu, L. Jiang, A. A. Clerk, and J. G. E. Harris. Nonreciprocal control and cooling of phonon modes in an optomechanical system. Nature 568(7750), 65-69 (2019)

[138] C. F. Ockeloen-Korppi, E. Damskägg, J.-M. Pirkkalainen, M. Asjad, A. A. Clerk, F. Massel, M. J. Woolley, and M. A. Sillanpää. Stabilized entanglement of massive mechanical oscillators. Nature 556(7702), 478-482 (2018)

[139] E. Gil-Santos, M. Labousse, C. Baker, A. Goetschy, W. Hease, C. Gomez, A. Lemaître, G. Leo, C. Ciuti, and I. Favero. Light-mediated cascaded locking of multiple nano-optomechanical oscillators. Phys. Rev. Lett. 118, 063605 (2017)

[140] J. Sheng, X. Wei, C. Yang, and H. Wu. Self-organized synchronization of phonon lasers. Phys. Rev. Lett. 124, 053604 (2020)

[141] R. Leijssen, G. R. La Gala, L. Freisem, J. T. Muhonen, and E. Verhagen. Nonlinear cavity optomechanics with nanomechanical thermal fluctuations. Nature Communications 8(1), ncomms16024 (2017)

[142] G. S. MacCabe, H. Ren, J. Luo, J. D. Cohen, H. Zhou, A. Sipahigil, M. Mirhosseini, and O. Painter. Nano-acoustic resonator with ultralong phonon lifetime. Science 370(6518), 840 (2020) 
[143] P. Kharel, Y. Chu, M. Power, W. H. Renninger, R. J. Schoelkopf, and P. T. Rakich. Ultra-high-Q phononic resonators on-chip at cryogenic temperatures. $A P L$ Photonics 3(6), 066101 (2021)

[144] L. Mercadé, K. Pelka, R. Burgwal, A. Xuereb, A. Martínez, and E. Verhagen. Floquet phonon lasing in multimode optomechanical systems (2021), 2101.10788

[145] A. Pikovsky, M. G. Rosenblum, and J. Kurths. Synchronization, A Universal Concept in Nonlinear Sciences (Cambridge University Press, Cambridge, 2001)

[146] D. Antonio, D. H. Zanette, and D. López. Frequency stabilization in nonlinear micromechanical oscillators. Nature Communications 3(1), 806 (2012)

[147] A. I. Kuznetsov, A. E. Miroshnichenko, M. L. Brongersma, Y. S. Kivshar, and B. Luk'yanchuk. Optically resonant dielectric nanostructures. Science 354(6314), aag2472 (2016)

[148] G. Grinblat, Y. Li, M. P. Nielsen, R. F. Oulton, and S. A. Maier. Enhanced third harmonic generation in single germanium nanodisks excited at the anapole mode. Nano Lett. 16(7), 4635-4640 (2016)

[149] O. Hess, J. B. Pendry, S. A. Maier, R. F. Oulton, J. M. Hamm, and K. L. Tsakmakidis. Active nanoplasmonic metamaterials. Nature Materials 11(7), 573-584 (2012)

[150] W. Bogaerts, P. De Heyn, T. Van Vaerenbergh, K. De Vos, S. Kumar Selvaraja, T. Claes, P. Dumon, P. Bienstman, D. Van Thourhout, and R. Baets. Silicon microring resonators. Laser \& Photon. Rev. 6(1), 47-73 (2021)

[151] I. Volkovskaya, L. Xu, L. Huang, A. I. Smirnov, A. E. Miroshnichenko, and D. Smirnova. Multipolar second-harmonic generation from high-Q quasi-BIC states in subwavelength resonators. Nanophotonics 9(12), 3953-3963 (2020)

[152] C. W. Hsu, B. Zhen, A. D. Stone, J. D. Joannopoulos, and M. Soljačić. Bound states in the continuum. Nature Reviews Materials 1(9), 16048 (2016)

[153] Z. F. Sadrieva, I. S. Sinev, K. L. Koshelev, A. Samusev, I. V. Iorsh, O. Takayama, R. Malureanu, A. A. Bogdanov, and A. V. Lavrinenko. Transition from optical bound states in the continuum to leaky resonances: Role of substrate and roughness. ACS Photonics 4(4), 723-727 (2017)

[154] M. F. Limonov, M. V. Rybin, A. N. Poddubny, and Y. S. Kivshar. Fano resonances in photonics. Nature Photonics 11(9), 543-554 (2017)

[155] L. Babić and M. J. A. de Dood. Interpretation of Fano lineshape reversal in the reflectivity spectra of photonic crystal slabs. Opt. Express 18(25), 26569-26582 (2010)

[156] C. Baker, W. Hease, D.-T. Nguyen, A. Andronico, S. Ducci, G. Leo, and I. Favero. Photoelastic coupling in gallium arsenide optomechanical disk resonators. Opt. Express 22(12), 14072-14086 (2014)

[157] S. Adachi. GaAs, AlAs, and AlxGa1-xAs: Material parameters for use in research and device applications. Journal of Applied Physics 58(3), R1-R29 (1985) 
[158] E. Gil-Santos, C. Baker, D. T. Nguyen, W. Hease, C. Gomez, A. Lemaître, S. Ducci, G. Leo, and I. Favero. High-frequency nano-optomechanical disk resonators in liquids. Nature Nanotechnology 10(9), 810-816 (2015)

[159] R. Van Laer, B. Kuyken, D. Van Thourhout, and R. Baets. Interaction between light and highly confined hypersound in a silicon photonic nanowire. Nature Photonics 9(3), 199-203 (2015)

[160] A. H. Safavi-Naeini, J. T. Hill, S. Meenehan, J. Chan, S. Gröblacher, and O. Painter. Two-dimensional phononic-photonic band gap optomechanical crystal cavity. Phys. Rev. Lett. 112, 153603 (2014)

[161] W. Yan, P. Lalanne, and M. Qiu. Shape deformation of nanoresonator: A quasinormal-mode perturbation theory. Phys. Rev. Lett. 125, 013901 (2020)

[162] L. Mercadé, n. Barreda, and A. Martínez. Dispersive optomechanics of supercavity modes in high-index disks. Opt. Lett. 45(18), 5238-5241 (2020)

[163] H. Ren, M. H. Matheny, G. S. MacCabe, J. Luo, H. Pfeifer, M. Mirhosseini, and O. Painter. Two-dimensional optomechanical crystal cavity with high quantum cooperativity. Nature Communications 11(1), 3373 (2020)

[164] E. P. Ippen and R. H. Stolen. Stimulated Brillouin scattering in optical fibers. Appl. Phys. Lett. 21(11), 539-541 (1972)

[165] J. Capmany and D. Novak. Microwave photonics combines two worlds. Nature Photonics 1(6), 319-330 (2007)

[166] B. Vidal, M. A. Piqueras, and J. Martí. Tunable and reconfigurable photonic microwave filter based on stimulated Brillouin scattering. Opt. Lett. 32(1), 23-25 (2007)

[167] Z. Zhu, D. J. Gauthier, and R. W. Boyd. Stored light in an optical fiber via stimulated Brillouin scattering. Science 318(5857), 1748-1750 (2007)

[168] A. Choudhary, B. Morrison, I. Aryanfar, S. Shahnia, M. Pagani, Y. Liu, K. Vu, S. Madden, D. Marpaung, and B. J. Eggleton. Advanced integrated microwave signal processing with giant on-chip Brillouin gain. Journal of Lightwave Technology 35(4), 846-854 (2017)

[169] D. Marpaung, B. Morrison, M. Pagani, R. Pant, D.-Y. Choi, B. Luther-Davies, S. J. Madden, and B. J. Eggleton. Low-power, chip-based stimulated Brillouin scattering microwave photonic filter with ultrahigh selectivity. Optica 2(2), 76-83 (2015)

[170] R. V. Laer, A. Bazin, B. Kuyken, R. Baets, and D. V. Thourhout. Net on-chip Brillouin gain based on suspended silicon nanowires. New Journal of Physics 17(11), 115005 (2015)

[171] N. T. Otterstrom, R. O. Behunin, E. A. Kittlaus, Z. Wang, and P. T. Rakich. A silicon Brillouin laser. Science 360(6393), 1113-1116 (2018) 
[172] C. J. Sarabalis, J. T. Hill, and A. H. Safavi-Naeini. Guided acoustic and optical waves in silicon-on-insulator for Brillouin scattering and optomechanics. APL Photonics 1(7), 071301 (2016)

[173] S. Gundavarapu, G. M. Brodnik, M. Puckett, T. Huffman, D. Bose, R. Behunin, J. Wu, T. Qiu, C. Pinho, N. Chauhan, J. Nohava, P. T. Rakich, K. D. Nelson, M. Salit, and D. J. Blumenthal. Sub-hertz fundamental linewidth photonic integrated Brillouin laser. Nature Photonics 13(1), 60-67 (2019)

[174] F. Gyger, J. Liu, F. Yang, J. He, A. S. Raja, R. N. Wang, S. A. Bhave, T. J. Kippenberg, and L. Thévenaz. Observation of stimulated Brillouin scattering in silicon nitride integrated waveguides. Phys. Rev. Lett. 124, 013902 (2020)

[175] C. Wolff, R. Soref, C. Poulton, and B. Eggleton. Germanium as a material for stimulated Brillouin scattering in the mid-infrared. Opt. Express 22(25), 3073530747 (2014)

[176] Y. Liu, N. Dostart, and M. A. Popovi'c. Toward microphononic circuits on chip: An evaluation of components based on high-contrast evanescent confinement of acoustic waves. arXiv: Applied Physics (2017)

[177] R. Vogelgesang, M. Grimsditch, and J. S. Wallace. The elastic constants of single crystal $\beta$-Si3N4. Appl. Phys. Lett. 76(8), 982-984 (2000)

[178] W. Jin, R. G. Polcawich, P. A. Morton, and J. E. Bowers. Piezoelectrically tuned silicon nitride ring resonator. Opt. Express 26(3), 3174-3187 (2018)

[179] L. Mercadé, A. V. Korovin, Y. Pennec, J. Ahopelto, B. Djafari-Rouhani, and A. Martínez. Vertical engineering for large Brillouin gain in unreleased siliconbased waveguides. Phys. Rev. Applied 15, 034021 (2021)

[180] Q. Fan, C. Chai, Q. Wei, P. Zhou, and Y. Yang. Elastic anisotropy and electronic properties of Si3N4 under pressures. AIP Advances 6(8), 085207 (2021)

[181] R. M. de Ridder, K. Warhoff, A. Driessen, P. V. Lambeck, and H. Albers. Silicon oxynitride planar waveguiding structures for application in optical communication. IEEE Journal of Selected Topics in Quantum Electronics 4(6), 930-937 (1998)

[182] N. Tambo, Y. Liao, C. Zhou, E. M. Ashley, K. Takahashi, P. F. Nealey, Y. Naito, and J. Shiomi. Ultimate suppression of thermal transport in amorphous silicon nitride by phononic nanostructure. Sci Adv 6(39), eabc0075 (2020)

[183] D. Navarro-Urrios, N. E. Capuj, J. Maire, M. Colombano, J. Jaramillo-Fernandez, E. Chavez-Angel, L. L. Martin, L. Mercadé, A. Griol, A. Martínez, C. M. Sotomayor-Torres, and J. Ahopelto. Nanocrystalline silicon optomechanical cavities. Opt. Express 26(8), 9829-9839 (2018)

[184] D. Navarro-Urrios, M. F. Colombano, J. Maire, E. Chávez-Ángel, G. Arregui, N. E. Capuj, A. Devos, A. Griol, L. Bellieres, A. Martínez, K. Grigoras, T. Häkkinen, J. Saarilahti, T. Makkonen, C. M. Sotomayor-Torres, and J. Ahopelto. Properties of nanocrystalline silicon probed by optomechanics. Nanophotonics $\mathbf{9}(16)$, 4819 $4829(2020)$ 
[185] M. Mirhosseini, A. Sipahigil, M. Kalaee, and O. Painter. Quantum transduction of optical photons from a superconducting qubit (2020), 2004.04838

[186] M. Hossein-Zadeh and K. J. Vahala. Observation of injection locking in an optomechanical rf oscillator. Appl. Phys. Lett. 93(19), 191115 (2008)

[187] H. Ren, T. Shah, H. Pfeifer, C. Brendel, V. Peano, F. Marquardt, and O. Painter. Topological phonon transport in an optomechanical system (2020), 2009.06174 